\title{
QUANTUM CHAOS and ANALYTIC STRUCTURE of the SPECTRUM
}

by

ANTONIE ABRAHAM KOTZÉ

A thesis submitted to the Faculty of Science,

University of the Witwatersrand, Johannesburg, South Africa, in fulfillment of the requirements for the

Degree of Doctor of Philosophy.

Johannesburg

August 1992 


\begin{abstract}
Quantum chaos is associated with the phenomenon of avoided level crossings on a large scale which leads to a statistical behaviour similar to that of a Gaussian Orthogonal Ensemble (GOE) of matrices. The same behaviour is seen in a pure quantum one dimensional system consisting of a square well containing $\delta$-function barriers on a Cantor set. For a representative Hamiltonian of the form $H_{0}+\lambda H_{1}$, avoided level crossings are associated with branch point singularities when they are continued into the complex $\lambda$-plane. These are the exceptional points which are proposed to determine the form of the spectrum and thus the spectral fluctuation properties. It is prohibitive to determine the exact positions of the exceptional points but a procedure is given to determine their distribution and its implementation is demonstrated considering simple matrix models. By investigating the chaotic quartic oscillator the intricate connection between the distribution of exceptional points and the particular fluctuation properties of level spacings and associated eigenvector statistics is shown. The effect of the coupling matrix elements are also stressed. This means that a GOE, which has no physical input a priori, only simulates the behaviour of a chaotic system but states nothing as to the origin of this peculiar behaviour. The actual underlying mathematical mechanism that yields the special features of the spectrum is prescribed by the exceptional points in conjunction with the coupling matrix elements. This is called the exceptional point method. These results are substantiated by the chaotic system par excellence: the hydrogen atom in a strong uniform magnetic field. It is shown that the behaviour of this system only depends on the coupling matrix elements and the density of exceptional points. A measure is proposed that characterises the chaotic behaviour and it corresponds to the known classical and quantal behaviour. This leads to the conjecture that we must be able to retrieve the classical unstable periodic orbits and understand the deviations of $\bar{\Delta}_{3}$ from GOE and the scarring of the wave functions, seen when the system is analysed semiclassically, by investigating the density of exceptional points. We show that results from an exceptional point analysis follow that from a classical, semiclassical and quantum mechanical analysis. The exceptional points are therefore proposed as suitable quantities to build the bridge between the quantum mechanical and classical regimes. The exceptional point approach may then lead to a conclusive theory whereby quantum chaos can be characterised thoroughly.
\end{abstract}




\section{OORSIG}

Kwantumchaos word met vlakafstotings, wat op groot skaal in die energiespektrum voorkom, geassosiëer. Dit lei tot 'n statistiese gedrag soortgelyk aan die van 'n Gaussiese Ortogonale Ensemble (GOE) van matrikse. Dieselfde gedrag kom voor in 'n suiwer kwantum, eendimensionale sisteem wat uit 'n potensiaalput, met $\delta$-funksie versperrings op 'n Cantor versameling, bestaan. 'n Verteenwoordigende Hamiltoniaan $H_{0}+\lambda H_{1}$ se vlakafstotings word, in die komplekse $\lambda$-vlak, met vertakpuntsingulariteite geassosiëer. Dit is die uitsonderingspunte wat voorgehou word as die groothede wat die vorm van die spektrum, en dus die spektraalfluktuasie-eienskappe, bepaal. Dit is egter nie moontlik om die spesifieke posisies van die uisonderingspunte te bepaal nie maar 'n handelswyse word beskryf waardeur die verspreiding bepaal kan word. Die implementering van die proses word met twee eenvoudige matriksmodelle toegelig. Die ingewikkelde verhouding tussen die verspreiding van die uitsonderingspunte en die spesifieke fluktuasie-eienskappe, van die vlakspasiërings en die eievektor statistiek, word beskryf deur die kwartiese ossillator te ondersoek. Die uitwerking van die koppelingsmatrikselemente word ook omskryf. Dit beteken dat 'n GOE, waar geen $a$ priori fisiese insette bestaan nie, slegs die gedrag van 'n chaotiese sisteem naboots en niksseggend oor die oorsprong van die vreemde gedrag is nie. Die eintlike onderliggende wiskundige meganisme, wat tot die spesiale gedrag van die spektrum aanleiding gee, word deur die uitsonderingspunte, in samewerking met die koppelingselemente, voorgeskryf. Dit word die uitsonderingspuntmetode genoem. Hierdie resultate word verder onderskryf deur die chaotiese sisteem par excellence: die waterstofatoom in 'n sterk eenvormige magneetveld. Daar word gewys dat die gedrag van hierdie sisteem slegs van die koppelingselemente en die verspreiding van uitsonderingspunte afhang. 'n Maatstaf, wat die chaotiese gedrag kenmerk, word voorgestel en dié stem ooreen met die bekende klassieke en kwantum gedrag. Dit lei tot die vermoede dat die onbestendige klassieke periodiese bane verkry kan word deur die uitsonderingspunte te ondersoek. Die afwyking van $\bar{\Delta}_{3}$ van GOE en die brandmerking van die golffunksies, gesien wanneer die sisteem halfklassiek ondersoek word, moet dus ook deur die uitsonderingspuntformalisme beskryf kan word. Ons toon aan dat resultate van 'n uitsonderingspuntanalise dié van 'n klassieke, halfklassieke en kwantummeganiese ondersoek onderskryf. Die uitsonderingspunte word dus as geskikte groothede, om die brug tussen klassieke en kwantummeganika te bou voorgestel. Die uitsonderingspuntmetode kan dan tot 'n omvattende teorie, waardeur kwantumchaos behoorlik omskryf word, lei. 


\section{DECLARATION}

I declare that this dissertation is my own unaided work. It is being submitted for the degree of Doctor of Philosophy at the University of the Witwatersrand, Johannesburg, South Africa. It has not been submitted before for any degree or examination at any other University.

\section{VERKLARING}

Ek verklaar dat hierdie proefskrif my eie selfstandige werk is. Dit word voorgelê ter vervulling van die graad van Doktor in die Filosofie aan die Universiteit van die Witwatersrand, Johannesburg, Suid-Afrika. Dit is nog nie voorheen voorgelê ter vervulling van enige graad of eksamen aan enige universiteit nie.

Antonie Abraham Kotzé

dag van 1992. 


\section{ACKNOWLEDGEMENTS}

I wish to express sincerest gratitude to

- my supervisor Professor Dieter Heiss for his ever knowledgeable and valuable guidance, always unfailing encouragement and the inspiration derived from his contagious enthusiasm for physics,

- Antonella Sannino for all her help, the discussions we had over the past few years and for proofreading this thesis.

\section{DANKBETUIGINGS}

Hiermee wil ek my hartlike dank betuig teenoor

- my vrou Olga vir al haar ongesiene opofferings,

- my ouers vir die besondere en opregte opvoeding wat hul my gegee het, hul ondersteuning en aansporing,

- my kollegas by RAU wat altyd bereid was om te help. 
Hierdie proefskrif word opgedra aan my ouers

$\mathcal{J} \mathcal{A N}$ en $\mathcal{M} \mathcal{A R} \mathcal{T} \mathcal{I} \mathcal{K} O \mathcal{T} \mathcal{Z} \dot{\mathcal{E}}$ 


\section{Contents}

1 Introduction $\quad 1$

2 Chaos in Classical and Quantum Mechanics 9

2.1 Introduction . . . . . . . . . . . . . . . . . . . 10

2.2 Deterministic Chaos . . . . . . . . . . . . . . . 11

2.2.1 Classical Integrability . . . . . . . . . . . . . . . . 11

2.2 .2 Poincaré Surface of Section _ . . . . . . . . . . . . . . . . . . . . . . . . 12

2.2.3 Classical Lyaponov Exponent . . . . . . . . . . . . . . . . . 14

2.3 Quantisation of Integrable Systems . . . . . . . . . . . . . . . . . . 14

2.4 The Semiclassical Approach . . . . . . . . . . . . . . . . . . 15

2.4.1 Semiclassical Mechanics . . . . . . . . . . . . . 15

2.4 .2 Periodic Orbits . . . . . . . . . . . . . . . . . . . 18

2.4.3 Scarring of Eigenfunctions . . . . . . . . . . . . . . . 21

2.4.4 The Gutzwiller Trace Formula . . . . . . . . . . . . . . . 23

2.5 Experimental Evidence of Quantum Chaos . . . . . . . . . . . 25

3 Statistical Behaviour of Chaotic Systems 27

3.1 Introduction . . . . . . . . . . . . . . . . . . . . . . . . . 27

3.2 Statistical Analysis . . . . . . . . . . . . . . . . . . . . 28

3.2.1 Random Matrix Theories . . . . . . . . . . . . . . . 29

3.2.2 Gaussian Orthogonal Ensembles . . . . . . . . . . . . . 31

3.3 Level Repulsion in Spectra . . . . . . . . . . . . . . . . . . . . . . 31

3.4 Unfolding of Spectra and Weyl's Rule . . . . . . . . . . . . . . . 34

3.5 Level Correlations . . . . . . . . . . . . . . . . . . . . . 36

3.5.1 The Nearest Neighbour Spacing Distribution . . . . . . . 37

3.5.2 The Brody Parameter . . . . . . . . . . . . . . . . 38

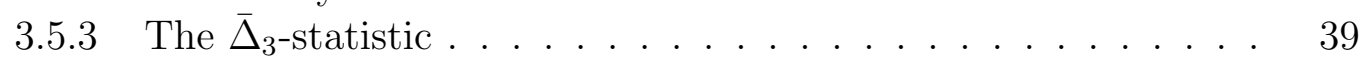

3.6 Spectral Analysis . . . . . . . . . . . . . . . . . . . . . . 41

3.6.1 The Autocorrelation Coefficients . . . . . . . . . . . . . 42

3.6.2 The Spectral Density Function . . . . . . . . . . . . . . . 43

3.7 Eigenfunction Statistics and Localisation . . . . . . . . . . . . . . . . . . . 45

3.8 Breaking of the Universality Regime . . . . . . . . . . . . . . 48 
3.9 Summary . . . . . . . . . . . . . . . . . . . . . . . . . . 49

4 Analytic Properties of the Spectrum $\quad 51$

4.1 Introduction . . . . . . . . . . . . . . . . . 51

4.2 The Form of the Hamiltonian . . . . . . . . . . . . . . . . . 52

4.3 Avoided Level Crossings . . . . . . . . . . . . . . . . . . . 53

4.4 Exceptional Points . . . . . . . . . . . . . . . . 55

4.4.1 Definition of Exceptional points . . . . . . . . . . 56

4.4 .2 Riemann Sheet Structure . . . . . . . . . . . . . . . 57

4.4.3 Parametric Dependence of Exceptional Points and the Line Spectrum . . . . . . . . . . . . . . 58

4.4.4 Definition of the Angles . . . . . . . . . . . . . . . 60

4.4.5 Effect of the Exceptional Points on the Spectrum . . . . . . 61

4.5 Density of Exceptional Points . . . . . . . . . . . . . . . 62

4.5.1 Line Spectrum: Nondegenerate Systems . . . . . . . . . . . . 63

4.5.2 Line Spectrum: Degenerate Systems . . . . . . . . . . . . . . 64

4.5.3 Distribution of Exceptional Points . . . . . . . . . . . . 65

4.6 Simple Examples . . . . . . . . . . . . . . . . . . 67

4.6.1 High density of exceptional points . . . . . . . . . . . . 67

4.6.2 Diluted density of exceptional points . . . . . . . . . . 70

4.7 Summary . . . . . . . . . . . . . . . . . . 71

5 The Quartic Oscillator $\quad \mathbf{7 5}$

5.1 Introduction . . . . . . . . . . . . . . . . . 75

5.2 The Model . . . . . . . . . . . . . . . . . . . . . 75

5.3 Classical Analysis . . . . . . . . . . . . . . . . . . . . 76

5.4 Matrix Representation of Quantum System . . . . . . . . . . . . . . 78

5.5 Spectrum of the Quartic Oscillator . . . . . . . . . . . . 81

5.6 Statistical Analysis . . . . . . . . . . . . . . . . . 83

5.6 .1 Spectral Statistics . . . . . . . . . . . . . . . 83

5.6 .2 Eigenvector Statistics . . . . . . . . . . . . . . . 85

5.7 Exceptional Points . . . . . . . . . . . . . . . . . 85

5.8 Periodic Orbit Analysis . . . . . . . . . . . . . . . . . . . . . 89

5.9 Summary . . . . . . . . . . . . . . . . . . . 91

6 The Hydrogen Atom in a Magnetic Field 93

6.1 Introduction . . . . . . . . . . . . . . . . . . . 93

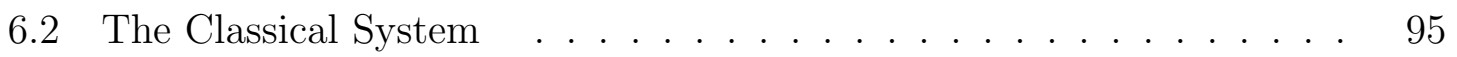

6.2.1 Constants of Motion . . . . . . . . . . . . . 95

6.2 .2 Scaling of the Hamiltonian . . . . . . . . . . . . . . . 95

6.2.3 Classical Poincaré Surface of Section . . . . . . . . . . . . . 96

6.3 The Eigenvalue Problem . . . . . . . . . . . . . . . . . . . . . . . . . 98 
6.3.1 The Coulomb Basis . . . . . . . . . . . . . . . . . . . 99

6.3.2 Sturmian Functions . . . . . . . . . . . . . . . . . 101

6.3.3 Matrix Representation with Sturmian Functions . . . . . . . . 102

6.4 Statistical Analysis . . . . . . . . . . . . . . . . . . . . 105

6.5 Exceptional Point Analysis . . . . . . . . . . . . . . . . . 108

6.5.1 Line Spectrum . . . . . . . . . . . . . . . . . . 108

6.5.2 Density of Exceptional Points . . . . . . . . . . . . . . 111

6.6 The Coupling Matrix Elements . . . . . . . . . . . . . . . . . . . 117

6.6.1 The Two Dimensional Matrix Problem . . . . . . . . . . 118

6.6.2 Generalisation to Higher Order . . . . . . . . . . . . . . . . . 119

6.7 A Quantitative Measure for Quantum Chaos . . . . . . . . . . . . . . 122

6.8 Periodic Orbits and Exceptional Points . . . . . . . . . . . . . . . 124

6.9 Summary . . . . . . . . . . . . . . . . . . . . 126

7 A One Dimensional Model for Quantum Chaos $\quad 127$

7.1 Introduction . . . . . . . . . . . . . . . . . . . 127

7.2 The Model . . . . . . . . . . . . . . . . . . . . . . . . . . . . . . 128

7.2 .1 The Classical System . . . . . . . . . . . . . . . . . . . . . 129

7.2 .2 The Quantum Mechanical System . . . . . . . . . . . . 129

7.3 The Spectrum . . . . . . . . . . . . . . . . . . . . . . . . . . . . 129

7.3.1 Asymptotic Behaviour . . . . . . . . . . . . . . . 130

7.3 .2 The Intermediate Spectrum _ . . . . . . . . . . . . . 131

7.4 Statistical Analysis . . . . . . . . . . . . . . . . . . 133

7.5 The Wave Functions . . . . . . . . . . . . . . . . . . . . . 135

7.6 Expectation Value of $x^{2} \ldots \ldots \ldots \ldots$

7.7 General Comment . . . . . . . . . . . . . . . . . . . 140

7.8 Summary . . . . . . . . . . . . . . . . . . . . . . . . . . . . . . 142

8 Summary and Conclusion $\quad 145$

A Quartic Oscillator: Matrix Representation $\quad 151$

A.1 Change of Variables . . . . . . . . . . . . . . . . . . . . . 151

A.2 Bose Operator Representation . . . . . . . . . . . . . . . . 153

A.2.1 Matrix Representation of the full Hamiltonian . . . . . . . . 153

A.2.2 Reduced Matrix Representation . . . . . . . . . . . . 155

B Position Operator: Matrix Representation $\quad 159$

B.1 Matrix Representation of Position Operator . . . . . . . . . . . . . . 159

B.2 Representation of Quartic Oscillator . . . . . . . . . . . . . . . 162

C Unfolding: Semiclassical Method 163 
D The Hydrogen Atom in a Magnetic Field 165

D.1 The Hamiltonian . . . . . . . . . . . . . . . . . . . . . . . 165

D.2 Schrödinger's Radial Integral . . . . . . . . . . . . . . . . . . 167

D.2.1 First Method . . . . . . . . . . . . . . . . 167

D.2.2 Second Method . . . . . . . . . . . . . . . 169

D.3 Sturmian Functions . . . . . . . . . . . . . . . . . . . . 170

D.4 Matrix Elements Using Sturmian Functions . . . . . . . . . . . . 173

D.4.1 The Radial Matrix Elements . . . . . . . . . . . . . . . . . . 173

D.4.2 The Ordinary Hydrogen Atom . . . . . . . . . . . . . . . . 175

D.5 Solving the Schrödinger Equation . . . . . . . . . . . . . 176

E One Dimensional Chaotic System 179

E.1 Schrödinger Method . . . . . . . . . . . . . . . . . . . . . 179

E.2 Matrix Diagonalisation . . . . . . . . . . . . . . . . . . . . 180

E.3 Numerical Accuracy . . . . . . . . . . . . . . . . . . . . . . . . . . . 181

E.4 Expansion Coefficients: Off-Center Interval . . . . . . . . . . . . . . 181

E.5 Expectation Value of $x^{2} \ldots \ldots \ldots$. . . . . . . . . . . . 182 


\section{List of Figures}

2.1 Poincaré surface of section for a chaotic trajectory. The development of the torus is clearly shown. The system is not fully chaotic and some trajectories are still on the torus (from $[$ Gl 87]). . . . . . . . . .

2.2 (a) Scarred eigenstates of the stadium with (b) the corresponding classical unstable periodic orbits (from [He 84]). . . . . . . . . . . .

2.3 Experimental eigenstates of the stadium with the corresponding classical periodic orbit. These eigenstates correspond to the theoretical states shown in Fig. (2.2) (from [SS 92]) . . . . . . . . . . .

3.1 Segments of complex spectra, each containing 50 levels and rescaled to the same spectrum span. The arrowheads mark pairs of levels with spacings smaller than one quarter of the average spacing $D$ (from $[\mathrm{BF} 81]) \ldots \ldots \ldots \ldots \ldots \ldots \ldots$

3.2 Nearest neighbour spacing histograms for the six cases of Figure (3.1), constructed by considering all the available levels instead of the 50 shown (from $[\mathrm{BF}$ 81]). . . . . . . . . . . . . . . . . .

3.3 (a) A staircase function $N(E)$ and its appropriate smooth function $N_{a v}(E)$. (b) The resultant level fluctuations. . . . . . . . . . . . . .

3.4 The nearest neighbour spacing distribution for Poisson (dashed curve), Wigner (solid) and Picket Fence (dotted) distributions. . . . . . . . .

$3.5 \bar{\Delta}_{3}$ for Poisson (dashed line), GOE (solid) and picket fence (dotted) spectra. . . . . . . . . . . . . . . . .

3.6 The autocorrelation coefficients for a GOE series. . . . . . . . . . . .

3.7 (a) Experimental spectrum for Hydrogen in a magnetic field. (b) The corresponding power spectrum with closed orbits correlated to the respective resonances (from [HM 88]; also see Chapter 6). . . . . . . .

3.8 Some localised and delocalised eigenfunctions for the kicked rotor. The probability $w_{n}=\left|\phi_{n}\right|$ is drawn on the vertical axis with the number of unperturbed states $n$ on the horizontal axis. $l_{H}$ is the localisation length (from $[$ Iz 90]) . . . . . . . . . . . . . . . . .

3.9 (i) Distribution of localisation lengths for (a) regular, (b) chaotic and (c) Poissonic systems. (ii) Mean localisation length $l_{d}$ for the kicked

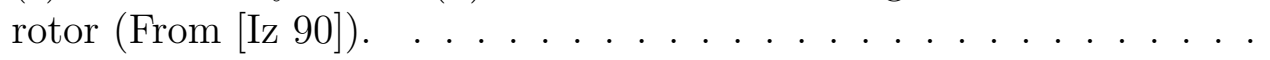


4.1 Two sheets representing energy levels as functions of the parameters $\lambda$ and $\kappa$ with a degeneracy $P$ at $\lambda^{*}, \kappa^{*}$ and energy $E^{*} . A$ is a path missing the degeneracy and $B$ is a path enclosing it (from [Be 81]). .

4.2 A near degeneracy at interaction strength $\lambda_{0}$. Although the levels do not cross, their corresponding wave functions interchange association at $\lambda_{0}($ from $[\mathrm{Be} 81]) . \ldots \ldots \ldots \ldots$

4.3 (a) Line spectrum of the unperturbed problem with a real degeneracy at $\lambda_{0}$. (b) The degeneracy is lifted when $\varphi$ is switched on and the wave functions interchange association. . . . . . . . . . . .

4.4 Asymptotic line spectrum for a system that has systematic degeneracies. Shown here is $E_{j}^{\prime}=\epsilon_{j}+\lambda \delta_{j}$ (solid lines), $E_{k}=\alpha_{k}+\lambda \omega_{k}$ (dashed lines) and the dotted lines represent the joining algebraic equations. .

4.5 Unperturbed spectrum of the example discussed in $\S 4.6 .1$ with all lines intersecting at $\lambda=1$ (from [HS 91a]) . . . . . . . . . . .

4.6 Average delocalisation length for $\varphi_{0}=0.003$ and a high density of exceptional points. . . . . . . . . . . . . . 69

4.7 Distribution of individual localisation lengths for $\lambda=1 \ldots$. . . . .

4.8 Unperturbed spectrum of the example discussed in $§ 4.6 .2$. The lines are randomly distributed (from $[\mathrm{HS}$ 91a]). . . . . . . . . . .

4.9 Average delocalisation length for $\varphi_{0}=0.05$ and the diluted density of exceptional points. . . . . . . . . . . . . . . .

4.10 Individual delocalisation length distribution of the diluted density of exceptional points at $\lambda=2$. The qualitative pattern persists for a large range of $\lambda$ values. . . . . . . . . . . . . . . . . . .

5.1 The potential in (5.2) for $E=1$ and (a) $\alpha=0$, (b) $\alpha=0.25$, (c) $\alpha=0.8$ and (d) $\alpha=1$ (from [CP 84]).

5.2 The Lyaponov exponent for the quartic oscillator. From this it is evident that chaos sets in at $\alpha \approx 0.15 \ldots \ldots \ldots$. . . . . . . .

5.3 Poincaré surface of section for the quartic oscillator for (a) $\alpha=0$, (b) $\alpha=0.25$, (c) $\alpha=0.8$ (the two obvious regular regions are outlined) and (d) $\alpha=0.93$ (from [CP 84]).

5.4 Portion of the lower part of the spectrum for the quartic oscillator with $-1 \leq \lambda \leq 1$ on the horizontal axis. . . . . . . . . . . . . . .

5.5 The nearest neighbour spacing distribution for (a) $\lambda=-1$, (b) $\lambda=0$, (c) $\lambda=0.8$ and (d) $\lambda=1$ where a bin size of 0.28 was used. The dashed curves relate to the Poisson and GOE results. . . . . . . . . .

5.6 The $\bar{\Delta}_{3}$-statistic (small circles) for (a) $\lambda=-1$, (b) $\lambda=0$, (c) $\lambda=0.8$ and (d) $\lambda=1$. The solid line and curve relate to the Poisson and GOE results. . . . . . . . . . . . . . . . .

5.7 Average delocalisation length for the quartic oscillator. $l_{d}$ is shown for $-3 \leq \lambda \leq 1 \ldots \ldots \ldots \ldots \ldots \ldots \ldots$ 
5.8 The distribution of individual delocalisation lengths for (a) $\lambda=-1$, (b) $\lambda=0$, (c) $\lambda=0.8$ and (d) $\lambda=1 \ldots \ldots \ldots$

5.9 Pattern of the unperturbed lines of the quartic oscillator. For the sake of clarity only a section of the spectrum for $N=76$ is shown. . . . . .

5.10 Number distribution of intersection points of the unperturbed lines for the quartic oscillator. The normalisation is arbitrary. There are further intersections for $\lambda$-values beyond the range displayed. . . . . .

5.11 Actual spectrum (full lines) and line spectrum (dashed lines) for $N=4$ $(M=6)$. The similarity between the two spectra when $\lambda \rightarrow \pm \infty$ is evident. . . . . . . . . . . . . . . .

5.12 Densities $|\Psi|^{2}$ of some eigenstates in the $A_{1}$ symmetry class where $\lambda=0.99$. The upper number is the number of the eigenstate and the lower number a scaled energy (see [EHP 89]).

5.13 Unstable periodic orbits for the same eigenstates shown in Fig. (5.12) where $\lambda=0.99$ (from $[$ EHP 89]) . . . . . . . . . . .

6.1 Equipotential lines of the potential in (6.6) in the $\tilde{\rho} \cdot \tilde{z}$ plane (from $[\mathrm{FW} 89]) \ldots \ldots \ldots \ldots \ldots \ldots$

6.2 Poincaré surface of section at $\varepsilon=-0.8,-0.5,-0.4,-0.3,-0.2,-0.1$ (from left to right and top to bottom [WH 89]). . . . . . . . . .

6.3 The spectrum for the hydrogen atom in a uniform magnetic field. The part shown here has $n=34,35, \ldots, 42$ with energy measured in Rydbergs. . . . . . . . . . . . . . . . .

6.4 Another part of the spectrum so that the avoided level crossings are discernible. Here $0.5 \times 10^{-8} \leq \lambda \leq 0.13 \times 10^{-7}$ and $-0.0006 \leq E \leq$ -0.0004 Ryds. . . . . . . . . . . . . . . . . .

6.5 (a) Nearest neighbour spacings distribution for a few values of the scaled energy showing the transition from Poisson to GOE behaviour. The solid line is the Brody fit. (b) $\bar{\Delta}_{3}$-statistic for a few values of $\varepsilon$

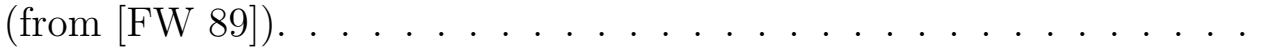

6.6 Line spectrum for the hydrogen atom in a magnetic field. For the sake of comparison the same $E$ and $\lambda$ values as that depicted in Fig. (6.3) are shown. . . . . . . . . . . . . . . .

6.7 Schematical representation of the "windows" (dashed rectangles) used to calculate the distribution of crossing points. The solid curves are those for constant $\varepsilon$ with values as indicated. . . . . . . . . . . .

6.8 Histograms depicting the density of crossing points for $-2.0 \leq \varepsilon \leq$ -0.8 and principle quantum numbers (a) $n^{*}=25$; and (b) $n^{*}=35$ using 40 bins for each. Note the similarity in form of the two histograms.114 
6.9 Three-dimensional graph depicting the density of crossing points $P$ as a function of $\varepsilon$ and $n^{*}$. Here we have the scaled energy $\varepsilon$ on the $x$-axis, the principle quantum number $n^{*}$ on the $y$-axis and $P$ on the $z$-axis. We note that the density of crossings declines with increasing $\varepsilon$. . . .

6.10 A geographical map (contourgraph) of Fig. (6.9) showing that the density of crossing points only depends on the scaled energy. The lines are lines of constant $P$ with the higher values on the left side of the graph. Evident is the declining nature of $P$ which is constant in $n^{*}$. .

6.11 Actual density of crossings $P^{\prime}$ as a function of $n^{*}$ shown for $\varepsilon=-0.2$. The solid line is a least squares fit given by $P_{\varepsilon}^{\prime}$. We listed $P_{\varepsilon}^{\prime}$ on the left side for a few values of $\varepsilon$. . . . . . . . . . . . . . . .

6.12 The coupling $\bar{C}$ as a function of $n$. The circles are the coupling due to the off-diagonal blocks and the diamonds due to the second off-diagonal blocks. The lines are numerical fits given by $\bar{C}_{1}^{\prime}$ and $\bar{C}_{2}^{\prime} \ldots \ldots$. . . .

6.13 The behaviour denoted by $\mathcal{B}^{\prime}$ as a function of $n$ where we use a logarithmic scale on the vertical axis. Each line corresponds to a different value of $\varepsilon$ as indicated with the corresponding $\mathcal{B}^{\prime}$ values listed on the right. . . . . . . . . . . . . . . . . . . .

7.1 The square well with $\delta$-function barriers on a Cantor set, of order $s=3$, with uniform length $\lambda$. The symmetry around the origin is obvious. .

7.2 The first 80 energy levels versus the coupling constant $\mu$ ranging from 0 to 4000 . We actually have $k_{n} / \pi$ on the vertical axis. . . . . . . . .

7.3 A blown-up version of the section in the little box in Fig. (7.2) showing the avoided level crossings. . . . . . . . . . . . . . .

7.4 (i) $\bar{\Delta}_{3}(L)$ (small circles) and (ii) nearest neighbour spacing distribution $P(S)$ for (a) $\mu=1000$ (b) $\mu=4000$ and (c) $\mu=10000$. The solid curves relate to the Poisson and GOE results. . . . . . . . . . . . .

7.5 Schematic illustration of the regular and chaotic regions of the spectrum as a function of the interaction strength. . . . . . . . . . .

7.6 The amplitudes of the positive parity eigenfunctions in the center interval versus $n$ in the order of increasing eigenenergies. The cases for (a) $\mu=1000$, (b) $\mu=4000$ and (c) $\mu=10000$ are shown. . . . . . .

7.7 The corresponding power spectra of the amplitudes shown in Fig. (7.6). The cases for (a) $\mu=1000$, (b) $\mu=4000$ and (c) $\mu=10000$ are shown. 138

7.8 The autocorrelation coefficients of the amplitudes in Fig. (7.6) for (a) $\mu=4000$ and (b) $\mu=10000$. The coefficients decay exponentially. . .

7.9 Expectation values of $x^{2}$ for (a) $\mu=1000$, (b) $\mu=4000$ and (c) $\mu=10000 \ldots \ldots \ldots \ldots \ldots \ldots \ldots$

A.1 The Hamiltonian $H$ is invariant under rotations and reflections of the square. $\tilde{q}_{1}^{\prime}$ and $\tilde{q}_{2}^{\prime}$ are the $\tilde{q}_{1}$ and $\tilde{q}_{2}$ axes rotated by $\pi / 4 \ldots \ldots$. . . 156 
D.1 The $l=0$ Sturmian functions for $n=1,2,3$ and 4 . Unlike hydrogen functions, the first node continues to move closer to the origin with increasing $n$ (from [Ro 70]). . . . . . . . . . . . . . . . . 172 


\section{List of Tables}

A.1 Character table for the $C_{4 v}$ symmetry group. . . . . . . . . . . 155 
God used beautiful mathematics in creating the world. Paul Dirac 


\section{Chapter 1}

\section{Introduction}

According to Newtonian mechanics all systems in nature are regular. This means that we can integrate the equations of motion and the dynamical system runs along a predictable and regular course that ends in some periodic or steady state. These systems are called integrable and their trajectories are confined to invariant tori. The special examples discussed in undergraduate physics courses create this erroneous impression. The general methods for solving more difficult problems are presented as technical refinements best left to the experts.

A century ago, however, mathematicians discovered that some apparently simple mechanical systems can have very complicated motions. Not only is their behaviour exceedingly sensitive to the precise starting conditions, but they never settle to any reasonable final state with a recognisable fixed pattern. Their movements look smooth over short time scales but they seem to jump unpredictably and indefinitely when we check their positions and momenta over large intervals. Astronomers became increasingly aware of this problem during the last 60 years but physicists began recognising it only some 20 years ago. This phenomenon now goes under the name of chaos that is an extensive research field [De 89].

Chaos denotes the irregular motion generated by nonlinear systems whose dynamical laws uniquely determine the time evolution of a state of a system from a knowledge of its previous history. Nonlinearity is a necessary but not a sufficient condition for the generation of chaotic motion. The observed chaotic behaviour in time is neither due to external sources of noise nor too infinitely many degrees of freedom. The actual source of irregularity is the property of the nonlinear system that initially close trajectories separate exponentially fast in a bounded region of phase space. It therefore becomes practically impossible to predict the long-time behaviour of these systems because in practice one can only fix their initial conditions with finite accuracy and errors increase exponentially fast [Sc 84], [LL 83].

Classical chaotic motion is intimately connected to the concept of periodic orbits, that is, solutions to the equations of motion that return to their initial conditions. Periodic orbits of an integrable system have a polynomial growth in contrast to an 
exponential growth in a chaotic system. As early as 1892 Poincaré pointed out that periodic orbits are densely distributed amongst all possible classical trajectories and that a study of them would provide the clue to the general behaviour of any mechanical system.

A well established and comprehensive theory has been set down since Lorentz rediscovered chaotic motion in 1963. A fundamental theorem is the KAM theorem that says that the motion in phase space of classical mechanics is neither completely regular nor completely irregular but the type of trajectory depends sensitively on the initial conditions. Classical chaos changed our intuition about the harmony and beauty of the universe. The existence of chaotic motion in classical conservative systems naturally leads to the question of how this irregularity manifests itself in the corresponding quantum systems? Will we observe the same irregularity? This research field has been named 'Quantum Chaos' and started to attract attention some 15 years ago.

Although much insight has been gained into the behaviour of quantum systems that have a classical chaotic counterpart, no conclusive theory has been formulated. The state of affairs can be summarised by the seven year old resumé of Gutzwiller which is to a large extent still applicable today [Gu 85]:

The word 'chaos' is not a technical term for the time being, in contrast to words such as ergodicity, mixing and entropy, which can be defined even if there are different kinds of each. Chaos, at this point, describes rather a vague feeling of malaise among scientists, an embarrassing state of affairs whose precise symptoms are not clear. This applies in particular to chaos in quantum mechanics; but in any situation, our recourse to chaos is almost an admission of failure about our efforts to find order and beauty in the description of nature.

Following this one can ask, what is 'Quantum Chaos' good for? Where is it needed? Can it be avoided? The answer is almost contradictory in terms: chaos is necessary to unify the following four basic areas of physics,

1. newtonian mechanics,

2. thermodynamics and equilibrium statistical mechanics,

3. atomic and molecular physics,

4. electromagnetism including optics.

Each of these separate fields was discovered and developed independently of the others. At this stage, nobody has any serious doubts about our understanding of these four (by now) classical areas based on the underlying mathematical principles. This situation is quite different from what prevails, e.g., in hydrodynamics, physics of condensed matter, nuclear and elementary particle physics. 
What happens if these classical areas come in contact with one another? Evidently we have to tie different ideas, pictures and mathematical structures together. The process of establishing the connection works in some very special circumstances but generally it runs into great difficulties. With only slight exaggeration, one always finds some kind of chaotic behaviour at the root of our troubles. Even greater problems arise when these four fields are quantised.

The last few decades has seen the emergence of two approaches for investigating the nature of the signature of 'Quantum Chaos'. The first is due to the believe that classical mechanics is quantum mechanics in the limit where Planck's constant vanishes and the second is a statistical approach that began with the study of nuclear energy levels. The first approach targets one of the most fundamental problems of physics today [Gu 90]: how can the classical mechanics of Newton, Euler and Lagrange be understood as a limiting case within the quantum mechanics of Heisenberg, Schrödinger and Dirac?

Einstein in 1917 was the first, and for 40 years the only, scientist to point out the true dimensions of this problem when the classical dynamical system is chaotic [Ei 17]. The Bohr-Sommerfeld quantisation rules state

$$
\frac{1}{2 \pi} \oint p_{i} d q_{i}=\hbar n_{i} ; \quad i=1,2, \ldots, N
$$

with $N$ the number of integrals of motion. Einstein was not satisfied with (1.1) because they were applicable only to the case of completely separable variables of the system. He pointed out that an absence of invariant tori in phase space would prevent any use of (1.1) on the corresponding classical system so that it is only applicable to integrable systems. This problem is so fundamental and complicated that a general quantisation procedure has not yet been established. The main obstacle is our inability to provide an effective quantal description for the chaotic trajectories in classical Hamiltonian systems.

During the last two decades periodic orbits have turned out to be of special significance in the transition from the classical to the quantum regime. Remnants of the classical unstable periodic orbits are seen in quantum chaotic systems. They are used as the link between the classical and quantum regimes. Selberg used periodic orbits to derive an equation that can be used to compare the classical and quantum representations of special systems [Oz 88]. This gave way to the Gutzwiller trace formula. It is today widely used in semiclassical mechanics to attempt to answer the question: how can classical mechanics give us any hints about the quantal energy levels when the classical system is chaotic [Gu 90]? Using the trace formula one can obtain the unstable periodic orbits from the quantum system and describe the localisation of the eigenfunctions around the unstable periodic orbits. This is known as scarring [He 84]. This motivates Chapter 2 which is a brief survey of aspects from semiclassical mechanics relevant to quantum chaos and this thesis.

The second approach (statistical analysis of spectra) emanated from the experi- 
mental and theoretical studies of nuclei. In the ground state energy region of the nuclear excitation spectrum where the level density is low, a detailed description of the individual levels is possible. However, at excitation energies of several $\mathrm{MeV}$, because of the high density, microscopic detail is relinquished. We thus come to rely on a statistical theory of energy levels that describes the general appearance and the degree of irregularity of the level structure. Such studies, whose nature is essentially different from the study of individual levels, are of interest because they reveal new features of the system. In the face of the complexity of nuclear systems, and the many degrees of freedom, Wigner turned the problem around. He wanted the spectrum of an object whose Hamiltonian is unknown, or more precisely, a Hamiltonian that is drawn at random from a large collection, subject only to the general restrictions of quantum mechanics [Wi 51]. This method is today known as Random Matrix Theories [BF 81]. There is thus no physical input a priori yet much success was achieved in describing nuclear spectra with high level density. Physicists extended this method to other systems where their fluctuation properties were compared to those of random matrices. It was found that the level fluctuations of many physical systems are similar to those of random Hamiltonians. This is unexpected because the agreement cannot be due to a presumed random character of the matrix elements of the studied systems.

Random matrix models might have quite different global properties but the local fluctuation properties are always the same and determined only by the overall symmetries of the system [BG 84]. These symmetries put important restrictions on the admissible matrix ensembles that can be used to describe the behaviour of a system. All the systems described in this thesis are time-reversal invariant so that the Hamiltonians are real symmetric and the random matrices can be chosen Gaussian Orthogonal. A Gaussian Orthogonal Ensemble (GOE) of matrices exhibits many features ascribed to chaotic systems [Bo 91]. This gave way to the hypothesis that the relationship between time reversal invariant systems, whose classical counterpart is chaotic, and the GOE is universal. Random Matrix Theories and the relevant statistical measures used in characterising the statistical behaviour of a system are outlined in Chapter 3.

The level fluctuations in chaotic spectra are due to level repulsions (avoided level crossings) in the spectrum [BG 84]. To study level repulsion at least one parameter is required to visualise the repelling levels [Be 85]. A Hamiltonian of the form $H(\lambda)=$ $H_{0}+\lambda H_{1}$, the starting point of many investigations in all branches of physics, is thus suitable. It is assumed that the two Hamiltonians $H_{0}$ and $H_{1}$ are regular that is without fluctuations, therefore, as the strength parameter $\lambda$ is varied between $\lambda=0$ and $\lambda \rightarrow \infty$, a transition from order to order is seen. If the symmetries relating to $H_{0}$ and $H_{1}$ are incompatible or simply if the two operators do not commute, the mixed problem is usually characterised by many avoided level crossings. Genuine degeneracies are excluded by assuming that the full Hamiltonian is irreducible with respect to internal symmetries. Each level repulsion is associated with a square root 
branch point singularity when the spectrum is continued into the complex $\lambda$-plane. These are known as exceptional points and are specific to the particular Hamiltonian under scrutiny [Ka 66]. The definition of exceptional points is general and applies to operators in an infinite dimensional space also to spectra of operators with a continuum contribution.

Globally the exceptional points determine the shape of the spectrum and thus the fluctuation properties [HS 91b]. Determining the exact positions of these branch point singularities is impractical for large systems. It is shown, however, that the positions of the exceptional points in the complex $\lambda$-plane are determined by $H_{0}$ and $H_{1}$ only. It is also possible to obtain information on their distribution and density from knowledge of these two operators alone [HS 91a], [HK 91]. The connection between the distribution of exceptional points and the emergence of quantum chaos is seen as the fundamental mechanism that produces quantum chaos as far as operators of the form $H_{0}+\lambda H_{1}$ are concerned.

There are two physical quantities that determine the spectral behaviour of a system: the density of states and the coupling matrix elements [GW 90]. In a chaotic system there is a relation between the density of states and the density of exceptional points in that the higher the one, the higher the other; the precise theoretical relation is not yet known [KH 92b]. The theme of this thesis is to characterise the chaotic behaviour of a system by investigating the exceptional points, that is, the underlying mathematical mechanisms and common structures of quantum mechanical operators that give rise to fluctuations characteristic of quantum chaos. Within this investigation, the role of the coupling matrix elements is thoroughly considered. This is termed the exceptional point method and is put forward as a third approach to the study of quantum chaotic systems.

As stated earlier, the exceptional points determine the shape of the spectrum where a chaotic spectrum leads to a GOE statistical behaviour. Exceptional points are also essential for the occurrence of phase transitions in finite Fermi systems [He 88], [HK 88b] where Wu, Feng and Vallièrs showed that GOE level statistics occur in the quantum phase transitional region [WF 90]. The exceptional point approach thus explains the statistical GOE behaviour well. On the other hand, the periodic orbits are obtained semiclassically, with the use of Gutzwiller's trace formula, from hidden periodicities in the quantal spectrum. It is thus conjectured that the substructure found in the spectrum and the eigenfunctions relating to the unstable periodic orbits of an associated classical system must therefore be related to the exceptional points. The fact that GOE behaviour is detected in quantum chaotic systems is just a fluke of nature and Random Matrix Theories cannot be used to obtain the origin of this behaviour. This is substantiated by the fact that the $\bar{\Delta}_{3}$-statistic does not follow the GOE results, exactly, over large intervals. The deviations can be explained with the Gutzwiller trace formula reinforcing the conjecture that the origin of the behaviour should be sought in the exceptional points. The aim of this thesis is now twofold: the first is to relate the density of exceptional points of the underlying Hamiltonian 
to the occurrence or absence of spectral fluctuations characteristic of quantum chaos or regular behaviour respectively. This will reconcile the statistical approach to the exceptional point method. The findings are believed to be generic for quantal spectra even if no classical analogue is available. The second aim is to give the first steps into obtaining the periodic orbits and explain scarring of the wave functions with the density of exceptional points. We believe that a thorough investigation will also reconcile the semiclassical approach with the exceptional point method.

A detailed introduction to the idea of exceptional points and the exceptional point approach is given in Chapter 4 . We introduce an efficient method to obtain the density of exceptional points. Use is made of the conjecture that the chaotic spectra discussed in this thesis have simple substructures that can be extracted. This can, however, only be achieved if a representation where $H_{0}$ is a diagonal matrix is used. Simple mathematical models are used to aid the understanding of these branch point singularities and the associated Riemann sheet structure. Using this knowledge, the density of exceptional points of the chaotic quartic oscillator is calculated and compared to the known statistical behaviour in Chapter 5. The behaviour of the eigenvectors is also investigated in the chaotic regime. This investigation is given as first hand evidence of the connection between the statistical and exceptional point approaches.

Chapter 6 is a detailed analysis of the hydrogen atom in a strong uniform magnetic field. This is a practical application where the role of the exceptional points can be studied. This system has been described as the chaotic system par excellence because of its experimental significance. In this example the coupling matrix elements play a significant role. Although this system is complex, the underlying simple structure found in the spectrum gave way to the derivation of a measure that is used to characterise the behaviour. This gives physical impetus to the conjecture that the only quantities relevant in the characterisation of quantum chaos are the coupling matrix elements and the density of exceptional points. The last section is a first attempt to obtain a connection between the semiclassical and exceptional point approaches.

The second last chapter, Chapter 7, introduces a one dimensional quantal system that does not have a classical counterpart. This model was invented in the light of the fact that all one dimensional classical systems are integrable and thus nonchaotic meaning that their quantal counterparts also should not display chaotic behaviour. This chapter is slightly removed from the main theme of this thesis, but it serves to illustrate the fact that one dimensional quantum systems can display features ascribed to quantum systems having multidimensional classical chaotic counterparts. A thorough statistical analysis of the spectrum does then show a GOE behaviour. The eigenfunctions are also investigated and show aspects that are reminiscent of scarring. This association must, however, be seen in the light that this system does not have a classical analogue. The investigation is seen as evidence in part to the more general conjecture that in quantum mechanics any multidimensional system can be transformed into a one dimensional system. This transformation will, however, in the 
end lead to a potential that is complicated in nature and will always lead to chaotic behaviour [He 88]. It has also been implemented in one dimensional systems like the Lipkin model which is nonchaotic but shows chaotic behaviour after a rotation of the potential [Sa 91].

Thus being guided by examples that we believe are relevant and significant, we conjecture that the exceptional point method gives the underlying mathematical mechanism that could provide a bridge between the semiclassical and random matrix theory formalisms of quantum chaos. A summary of the conclusions drawn from this thesis is given in the concluding chapter. The appendices elucidate some of the mathematical rigour used in the thesis.

$æ$ 


\section{Chapter 2}

\section{Chaos in Classical and Quantum Mechanics}

Our understanding of the qualitative behaviour of classical dynamical systems has been improved by the discovery of the so-called 'deterministic chaotic motion' [Sc 84], [De 89]. Chaotic motion is characterised by an exponential instability of almost all orbits with respect to the initial conditions. In turn this instability leads to loss of memory of initial conditions, decay of correlations and an approach to statistical equilibrium [Ca 88].

The question whether the richness of chaotic behaviour persists, in some way, in quantum mechanics is of primary importance for the foundation of quantum statistical mechanics as well as for the explanation of several experimental results. At first sight the question seems to receive a negative answer because the spectra of classical systems are continuous while, bounded, conservative, finite particle number systems have discrete quantal spectra, no matter whether the corresponding classical systems are chaotic or not. This implies almost-periodicity in time of the wave functions and therefore an absence of chaotic behaviour. On the basis of Ehrenfest's theorem, however, one expects that, in the semiclassical region, a narrow wave packet will follow the classical trajectory and one is led to believe that quantum systems have different qualitative properties depending on whether the corresponding classical systems are integrable or chaotic.

The subject of 'Quantum Chaos' has been an active research field for more than a decade. Although a lot of experience and understanding has been accumulated, a detailed systematic theory is still lacking. It appears that the fascinating properties of systems that are the quantum mechanical analogues of classical chaotic systems still awaits thorough comprehension. 


\subsection{Introduction}

There are good reasons for studying quantal systems, whose classical behaviour are chaotic. They are more generic than the integrable ones. They reveal significant differences in the character of their wave functions, the distribution of their energy levels, the response to outside perturbations and related characteristics. Although many applications of quantum mechanics have had the primary purpose of solving special problems and comparing the results with experimental data, the concern with chaos is based on the hope of gaining some systematic awareness for all possibilities in quantum mechanics. Quantum mechanics may then become better appreciated in all its tremendous, but subtle, variety and some of its mysterious ambiguities may become better understood.

Since our physical intuition is so firmly grounded in classical mechanics, we have little choice but to advance as far as we can into the quantum regime along the trails that can be laid out with the help of classical mechanics. In the semiclassical limit $\hbar \rightarrow 0$ we can apply our understanding of classical dynamics to the study of wave functions, energy levels and other quantum mechanical entities. The behaviour that emerges must be distinguished from the strict classical limit because of the nonanalyticity of quantum mechanics at the limit $\hbar \rightarrow 0$ [Oz 88]. The semiclassical behaviour of quantum chaotic systems is currently an important research topic. This led to a better comprehension of these systems and many new features were discovered [Bo 92].

Percival proposed as early as 1973 that in the semiclassical limit, the quantal energy spectrum should consist of two parts with strongly contrasting properties: a regular and irregular part where the first is associated with the classical regular region, and the second with the irregular region in phase space [Pe 73]. Extensive statistical studies also found that the distribution of eigenvalues of classical chaotic systems fall into different universality classes depending on the symmetry of the system. These distributions have the same statistical properties as those of random matrices (see Chapter 3) and do not depend on the dynamics of the system nor on the particular type of interaction.

It is conjectured that the underlying mathematical mechanism linking the universal fluctuations, seen both in the semiclassical treatment and random matrix theories, are the exceptional points and the coupling matrix elements as far as operators of the type $H_{0}+\lambda H_{1}$ are concerned. We conjecture that there is a link between the periodic orbits and the exceptional points such that the patterns seen as scars of periodic orbits will also emerge through the Riemann sheet structure and distribution of the exceptional points (see Chapters 4 and §6.5) [HC 92]. It is the aim of this thesis to examine the distribution of these singularities, associated with the energy levels and the corresponding spectral fluctuation properties, in correspondence to the role of the coupling matrix elements, thus providing a bridge between the semiclassical treatment and the statistical analysis of chaotic systems. 
In this chapter, a brief survey of a selection of results, relevant to this thesis will be presented. To render the thesis self-contained, the first part will introduce a few basic concepts from 'deterministic chaos'. Because classical chaos is such a vast expanding field of research, the description will be brief and covers only the relevant quantities, that will be referred to later on $^{1}$. The second part briefly introduces semiclassical mechanics because of its current central importance to the study of quantum chaos. Various quantities such as periodic orbits, scars and the Gutzwiller trace formula are outlined. The last part gives an overview of the recent advances made in the experimental detection of 'Quantum Chaos'.

\section{$2.2 \quad$ Deterministic Chaos}

For centuries, after the discovery of Newton's equations of motion, it has been essentially impossible to go beyond the two-body Kepler problem and systems of harmonic oscillators. The main way to analyse the behaviour of many-body systems, interacting via nonlinear forces, was the use of perturbation theory. It was, however, soon realised that resonance phenomena rendered the traditional perturbation methods divergent.

It was in this connection that great significance was attached to the proof of Poincaré that, under very general conditions, any perturbation of an integrable system will destroy all the analytical constants of motion, except the energy. This was considered as a convincing argument in favour of the ergodic hypothesis and of the justification for equilibrium classical statistical mechanics. Recently, two major achievements led to a new and better understanding of the qualitative features of classical motion [Ar 80]:

- The Kolmogorov, Arnold and Moser (KAM) theorem states that small perturbations of an integrable system leave the system close to the integrable system and most orbits remain quasi-periodic. As the strength of the perturbation is increased, the system undergoes a gradual transition from near-integrable to ergodic motion.

- Classical dynamical systems, governed by purely deterministic laws, may exhibit a purely random motion despite the seemingly contradiction of these terms.

To quantify classical chaotic behaviour we outline a few relevant concepts in the following subsections.

\subsubsection{Classical Integrability}

Integrable dynamical systems are characterised by the existence of constants of motion in addition to the energy. We consider Hamiltonian systems with $N$ degrees of

\footnotetext{
${ }^{1}$ See the book by Bai-Lin [Ba 90] for reprints of the most important papers in this field.
} 
freedom. If the Hamilton-Jacobi equations are separable into $N$ independent equations, one for each degree of freedom, then we say that the Hamiltonian, and resulting motion, is integrable. The separation constants are known as isolating integrals.

A conservative Hamiltonian system, with $N$ degrees of freedom, is called completely integrable if it possesses $N$ (functionally) independent single valued analytic first integrals, which commute with respect to the associate Poisson bracket [SL 86]. The energy stays constant so that all trajectories are confined to a compact $N$ dimensional manifold in the $2 N$-dimensional phase-space. The topology of this manifold is the one of an $N$-dimensional torus [Ec 88], [LL 83].

We also say that a time-independent Hamiltonian system is integrable if there is a canonical transformation $S(\mathbf{q}, \mathbf{J})$ to two new variables $\theta$ and $\mathbf{J}$ (the so-called action angle variables) viz.

$$
\mathbf{q}, \mathbf{p}=\frac{\partial S(\mathbf{q}, \mathbf{J})}{\partial \mathbf{q}} \leftrightarrow \mathbf{J}, \theta=\frac{\partial S(\mathbf{q}, \mathbf{J})}{\partial \mathbf{J}}
$$

and the Hamiltonian is independent of $\mathbf{J}$. The action-angle variables give the complete solution of the equations of motion.

This is in contrast to what happens in nonintegrable systems. Here, an arbitrary chosen piece $W$ of phase space, with an initial simple shape, gets progressively deformed into a tangle of interwoven arms and branches, while staying simply connected and keeping the same volume (Liouville's theorem) on a constant energy surface [Ma 92].

The following can be stated:

- If a classical system is integrable, e.g. all the constants of motion can be found, the motion is always regular and all the trajectories lie on invariant tori.

- If a system is classical and nonintegrable, the motion might not be regular and the system might be chaotic when the trajectories diverge exponentially in phase-space. For a fully chaotic system there are no invariant tori and a single generic trajectory covers the whole energy shell uniformly.

\subsubsection{Poincaré Surface of Section}

The trajectories in phase space often have very involved global features which are in apparent contradiction to the locally smooth flow. Poincaré proposed to deal with this problem by intersecting the flow at discrete times. The definition of a Poincaré Surface of Section thus lies at the heart of the treatment of Hamiltonian flows [LL 83], [Gl 87].

The surface of section is especially useful in systems with two degrees of freedom, because the surface of section has two dimensions and the map can be visualised rather easily. For an autonomous system with two degrees of freedom, the phase space is four dimensional. The trajectory in phase space is then given by

$$
\mathbf{x}(t)=\left[p_{1}(t), p_{2}(t), q_{1}(t), q_{2}(t)\right]
$$


Figure 2.1: Poincaré surface of section for a chaotic trajectory. The development of the torus is clearly shown. The system is not fully chaotic and some trajectories are still on the torus (from [Gl 87]).

The trajectory lies on a three dimensional energy surface $H\left(p_{1}, p_{2}, q_{1}, q_{2}\right)=H_{0}$ in the four dimensional phase space. This equation determines any of the four variables in terms of the others, say

$$
p_{2}=p_{2}\left(p_{1}, q_{1}, q_{2}\right)
$$

We are therefore led to consider the projection of the trajectory onto a three dimensional volume $\left(p_{1}, q_{1}, q_{2}\right)$. If the motion is bounded, the plane $q_{2}=$ constant, within this volume, may be crossed, repeatedly, by the trajectory. This plane, consisting of a single coordinate $q_{1}$, and its canonical momentum $p_{1}$, is a convenient choice for the surface of section. The time evolution and Poincaré section of a particular chaotic orbit is shown in Fig. (2.1). If a constant of motion exists in addition to $H_{0}$, then $p_{1}=p_{1}\left(q_{1}, q_{2}\right)$ and the successive crossings with the surface of section must lie on a unique curve. The existence of constants of motion can thus be determined in principle. This procedure is, however, not recommended in practice.

A regular or integrable system will have a Poincaré map showing a unique pattern where only a fraction of the area of the plane is filled with crossing points. A chaotic system's trajectory traverses nearly every single point on the plane but periodic orbits can still occur; these orbits form a set of measure zero. The surface of section is used to show how the orderly structure of phase space is lost when an integrable system is perturbed so as to undergo a transition to a chaotic one. 


\subsubsection{Classical Lyaponov Exponent}

Nonlinearity is a necessary, but not a sufficient condition for the generation of chaotic motion. This chaotic motion is neither due to external sources or noise, nor to an infinite number of degrees of freedom. The actual source of irregularity is the property of the nonlinear system of separating initially close trajectories exponentially fast in a bounded region. It therefore becomes impossible to predict the long-time behaviour of these systems because, in practice, one can only fix their initial conditions with finite accuracy and errors increase exponentially fast.

The Lyaponov exponent is a measure of the exponential divergence of initially close trajectories where

$$
\lambda=\lim _{t \rightarrow \infty} \frac{1}{t} \ln \left(\frac{d(t)}{d(0)}\right)
$$

where $d(t)$ is the distance between two initially close orbits and $t$ is the time. It also measures the average loss of information about the position of a point. This exponent is zero for integrable systems (no divergence of trajectories) and larger than zero for chaotic systems (divergence of trajectories) which means that almost all trajectories are random, unpredictable and uncomputable for arbitrary long times.

\subsection{Quantisation of Integrable Systems}

The quantisation of classical integrable systems relies explicitly on the invariant tori that foliate phase space. This was realised by Einstein who modified the BohrSommerfeld quantisation rules in (1.1) to read [Pa 90]

$$
\int_{C_{j}} p d q=2 \pi \hbar n_{j}
$$

where the contour $C_{j}$ can be any closed loop on an invariant torus and $n_{j} \geq 0$ are integers. This shows that only integrable systems can be quantised in this manner. If a system is also separable, (2.1) can again be modified such that

$$
\int_{C_{j}} p d q=2 \pi \hbar\left(n_{j}+\frac{\beta_{j}}{4}\right)
$$

where $\beta_{j} \geq 0$ are integers. The energy spectrum for an integrable system is now given explicitly by the equations

$$
E_{j}=H\left[\hbar\left(n_{j}+\frac{\beta_{j}}{4}\right)\right]
$$

so that the eigenenergies correspond to level surfaces of the Hamiltonian $H . \beta_{j}$ is called a Maslov index and can be understood as the number of conjugate points, or Morse index, for the trajectory. The quantisation condition (2.2) has been given the name EBK quantisation after Einstein, Brillouin and Keller [Oz 88]. 
If the system is integrable, the motion is confined to a Lagrangian manifold such that the Maslov index is a topological invariant of this manifold. The Maslov index is determined by the topology of this manifold, in phase space, with respect to position space. This more general perspective is used to define Morse indices for nonintegrable systems provided that one studies the Lagrangian manifold which is generated by a particular trajectory and its neighbours in phase space [Gu 90].

Einstein's objection to the quantisation conditions of Bohr and Sommerfeld went unnoted for nearly 40 years. After it was 'rediscovered' it prompted people to look for a more general quantisation condition. To get a connection between classical and quantum mechanics use is made of the hypothesis that classical mechanics is quantum mechanics in the limit $\hbar \rightarrow 0$. This procedure is used to search for more general quantisation rules and falls under the heading of semiclassical mechanics.

\subsection{The Semiclassical Approach}

Classical mechanics has served humanity well for three centuries and still does. It has been confirmed to very high precision, particularly in celestial mechanics. The relevance, though, to very small systems like atoms and molecules has been severely questioned for about a 100 years. This prompted the advent of quantum mechanics which served to explain the problems where classical mechanics failed. The boundary between classical and quantal behaviour, however, lost some of its interest after Heisenberg's decisive results.

Although quantum mechanics has been the object of intensive studies, and has been confirmed by all experimental evidence available, it nevertheless remains somewhat a mystery. For this reason it is essential to understand as much of quantum mechanics as feasible, and as explicitly as possible, from a classical point of view; but can classical mechanics be understood as a limiting case of quantum mechanics? The trace formula of Gutzwiller for classical chaotic systems, seems to be the only general tool available, at this time, to establish a quantitive connection between the classical and quantal regimes.

\subsubsection{Semiclassical Mechanics}

Semiclassical mechanics is a wave mechanics based firmly on classical dynamics. It focuses all the attention on a quantum system's classical analogue. It is known that the fundamental tenets of quantum mechanics can not be derived from classical mechanics. Once they are known, however, it is natural to look for the opening that leads from the narrow classical confines to the wide open quantum fields.

This way of thinking was used by Van Vleck to write down a classical propagator [Gu 90]. Quantum mechanically the propagator $K\left(q^{\prime \prime}, q^{\prime}, t\right)$ is the value of the wave 
function in $q^{\prime \prime}$ at the time $t>0$ if it was concentrated in $q^{\prime}$ at the time $t=0$ so that ${ }^{2}$

$$
K\left(q^{\prime \prime}, q^{\prime}, t\right)=\sum_{j=0}^{\infty} \phi_{j}\left(q^{\prime \prime}\right) \phi_{j}^{\dagger}\left(q^{\prime}\right) \exp \left(-i E_{j} t / \hbar\right)
$$

In the energy representation the Green's function is defined to be

$$
G\left(q^{\prime \prime}, q^{\prime}, E\right)=\lim _{\epsilon \rightarrow 0} \sum_{j=0}^{\infty} \frac{\phi_{j}\left(q^{\prime \prime}\right) \phi_{j}^{\dagger}\left(q^{\prime}\right)}{E-E_{j}+i \epsilon}
$$

where the energy levels are an ordered set such that $E_{0} \leq E_{1} \leq \ldots \leq E_{j} \leq \ldots$ to each of which belongs a well defined normalised eigenfunction $\phi_{j}$. Van Vleck used this knowledge to obtain a crucial classical propagator, for a single trajectory that runs from $q^{\prime}$ to $q^{\prime \prime}$ in time $t=t^{\prime \prime}-t^{\prime}$, where

$$
K_{c}\left(q^{\prime \prime}, q^{\prime}, t\right)=(2 \pi i \hbar)^{-f / 2} \sqrt{C\left(q^{\prime \prime}, q^{\prime}, t\right)} \exp \left[\frac{i}{\hbar} R\left(q^{\prime \prime}, q^{\prime}, t\right)-i \phi\right]
$$

where $(2 \pi i \hbar)^{1 / 2}$ is always short for $(2 \pi \hbar)^{1 / 2} \exp (i \pi / 4)$. Further is $C$ the density of trajectories in phase space which started out in $\left(q^{\prime}, t^{\prime}\right)$ and are observed in $\left(q^{\prime \prime}, t^{\prime \prime}\right)$, after a time $t=t^{\prime \prime}-t^{\prime}$, such that

$$
C\left(q^{\prime \prime}, q^{\prime}, t\right)=\left|-\frac{\partial^{2} R}{\partial q_{i}^{\prime \prime} \partial q_{j}^{\prime}}\right|
$$

and $R$ is the Lagrangian action integral,

$$
R\left(q^{\prime \prime}, t^{\prime \prime}, q^{\prime}, t^{\prime}\right)=\int_{t^{\prime}}^{t^{\prime \prime}} d \tau L\left(\dot{q}_{0}, q_{0}, \tau\right) ; \quad t^{\prime} \leq \tau \leq t^{\prime \prime}
$$

with $L$ the Lagrangian, $f$ the number of degrees of freedom and $\phi$ a phase. The role of the propagator is to describe the evolution of the dynamical system at the time $t^{\prime \prime}$.

$K_{c}$ describes a wave which originates at $q^{\prime}$ and spreads to $q^{\prime \prime}$ such that

$$
K_{c}\left(q^{\prime \prime}, q^{\prime}, t\right) \simeq \int d q^{f} K_{c}\left(q^{\prime \prime}, q^{\prime}, t^{\prime \prime}-t\right) K_{c}\left(q, q^{\prime}, t-t^{\prime}\right)
$$

where $t^{\prime \prime}>t>t^{\prime}$ and the integral is evaluated by the stationary phase method.

In most situations, there is more than one trajectory going from $q^{\prime}$ to $q^{\prime \prime}$ in fixed time $t$. The classical propagator is then simply assumed to be

$$
K_{c}\left(q^{\prime \prime}, q^{\prime}, t\right)=\sum_{\text {class. traj. }}(2 \pi i \hbar)^{-f / 2} \sqrt{C\left(q^{\prime \prime}, q^{\prime}, t\right)} \exp \left[\frac{i}{\hbar} R\left(q^{\prime \prime}, q^{\prime}, t\right)-\frac{i \pi}{2} \nu\right]
$$

\footnotetext{
${ }^{2}$ This formulation is only valid if the potential is time-independent.
} 
with $\nu$ the number of conjugate points along the trajectory ${ }^{3}$ (Maslov index). Here, the superposition principle of quantum mechanics has been used but the computation of each individual term is still entirely classical. The result is thus simply the sum of the individual waves each following its own, mainly classical, trajectory. Equation (2.8) is the semiclassical approximation to the quantum propagator given in (2.3). This approximation is related to the stationary phase approximation near the classical trajectories [Bo 92].

The conversion from time to energy is accomplished by the transformation that defines the Green's function

$$
G\left(q^{\prime \prime}, q^{\prime}, E\right)=\int_{0}^{\infty} d t K\left(q^{\prime \prime}, q^{\prime}, t\right) \exp (i E t / \hbar)
$$

where we have omitted the subscript $c$ for $K$. The integral is a Laplace integral and $G$ is defined in the whole upper half of the complex energy plane. By inserting Van Vleck's formula (2.5) we obtain the semiclassical Green's function

$$
G_{c}\left(q^{\prime \prime}, q^{\prime}, t\right)=\frac{2 \pi}{(2 \pi i \hbar)^{\frac{f+1}{2}}} \sum_{\text {cl. tr. }} \sqrt{(-)^{f+1} D} \exp \left[\frac{i}{\hbar} S\left(q^{\prime \prime}, q^{\prime}, E\right)-\frac{i \pi}{2} \nu\right]
$$

with $D$ the density of trajectories defined to be

$$
D\left(q^{\prime \prime}, q^{\prime}, E\right)=-\frac{\partial^{2} S}{\partial E^{2}} C\left(q^{\prime \prime}, q^{\prime}, t\right)
$$

$S$ is the Hamilton-Jacobi action integral where

$$
S\left(q^{\prime \prime}, q^{\prime}, E\right)=R\left(q^{\prime \prime}, q^{\prime}, t\right)+E t
$$

or

$$
S\left(q^{\prime \prime}, q^{\prime}, E\right)=\int p d q=\int_{q^{\prime}}^{q^{\prime \prime}} \sqrt{2 m(E-V)}|d q|
$$

with the integral taken along the trajectory. Equation (2.10) is the semiclassical approximation (in the energy representation) to the quantum Green's function given in (2.4). The necessary condition for the applicability of the semiclassical formulae is

$$
\frac{S\left(q^{\prime \prime}, q^{\prime}\right)}{\hbar} \gg 1
$$

and if $\hbar \rightarrow 0$, only points where $\delta S\left(q^{\prime \prime}, q^{\prime}, E\right)=0$ will contribute to $G$. We stress the fact that equations (2.8) and (2.10) could not have been guessed without knowledge of the corresponding quantal relations.

Dirac was the first who thought to break the time interval from 0 to $t$ up into $N$ pieces of equal size so that the propagator from $q^{\prime}=q_{0}$ to $q^{\prime \prime}=q_{N}$ in (2.7) now

\footnotetext{
${ }^{3}$ Points along the trajectory where the semiclassical approximation should be modified.
} 
appears as an $(N-1)$-fold integral over all the intermediate positions $q_{1}, q_{2}, \ldots, q_{N-1}$. Feynman recognised that if the path is defined as a polygonal in position space to consist of the sequence of points

$$
\left(q^{\prime}, t^{\prime}\right)=\left(q_{0}, t_{0}\right),\left(q_{1}, t_{1}\right), \ldots,\left(q_{N-1}, t_{N-1}\right),\left(q_{N}, t_{N}\right)=\left(q^{\prime \prime}, t^{\prime \prime}\right),
$$

then the Lagrangian action for each path is well approximated by

$$
R_{N}=\sum_{k=1}^{N}\left(t_{k}-t_{k-1}\right) L\left(\frac{q_{k}-q_{k-1}}{t_{k}-t_{k-1}}, q_{k-1}, t_{k-1}\right) .
$$

Feynman's Path Integral for the propagator $K\left(q^{\prime \prime}, q^{\prime}, t\right)$ is then the expression

$$
\lim _{N \rightarrow \infty} \prod_{1}^{N}\left(\frac{m}{2 \pi i \hbar\left(t_{k}-t_{k-1}\right)}\right)^{f / 2} \int d q_{1} \ldots \int d q_{N-1} \exp \left(i R_{N} / \hbar\right)
$$

where the $(N-1)$-fold integral can be interpreted as adding up, independently, the contributions from all the possible paths that lead from $q^{\prime}$ to $q^{\prime \prime}$ in the given time interval $t^{\prime}$ to $t^{\prime \prime}$.

The path integral is the ideal tool for investigating what happens when $\hbar$ becomes small compared with the prevailing values of the Lagrangian action integral $R_{N}$. If the path is allowed to vary freely, the phase angle of the integrand in (2.15) goes many times through $2 \pi$, and the individual contributions destroy one another [Ec 88]. The destructive interference does not happen in the neighbourhood of those paths whose Lagrangian action does not change under small variations of the path. These are exactly the classical periodic orbits because the variational principle demands that the first variation of their Lagrangian actions vanish for any path which has the same initial and final values $q^{\prime}(t)$, i.e. which has a displacement such that $\delta q\left(t^{\prime}\right)=\delta q\left(t^{\prime \prime}\right)=$ 0 . The value of $R_{N}$ is then well approximated by the second variation.

In semiclassical mechanics (2.10) is interpreted, as far as feasible, as an approximation to (2.4). This is very difficult and can only be done for a few exceptional cases like the hydrogen atom. A reduced version, however, by taking the traces only, of (2.4) and (2.10), provides the most direct method of connecting classical and quantum mechanics. This leads to the known Gutzwiller trace formula which will be discussed in $§ 2.4 .4$.

\subsubsection{Periodic Orbits}

The idea of everything returning eventually to its point of departure has a strong hold on humanity. Kepler's discovery of the elliptical orbits for the planets seemed to give a scientific basis to this predilection for things running along a closed track and repeating their history over and over again.

Poicaré found that exactly periodic orbits, i.e. solutions of the equations of motion that return to their initial conditions, are densely distributed among all possible 
classical trajectories. He also suggested that a study of periodic orbits would provide the clue to the overall behaviour of any mechanical system. Periodic orbits also form continuous families in phase space that can be investigated by varying either the energy of the system, or some external parameter. By using the action-angle formalism, it is found that the number of periodic orbits has a polynomial growth for integrable systems. This is in sharp contrast to the exponential growth in a chaotic system. Periodic orbits show up in the surface of section as a finite set of, say, $n$ isolated points whose locations line up again on a simply closed curve.

To investigate the neighbourhood behaviour of a periodic orbit, we latch onto one particular periodic orbit with starting momentum and position $(\bar{p}, \bar{q})$. This orbit returns to this place after a time $\bar{T}$. We also assume that all trajectories that start at $\left(p^{\prime}, q^{\prime}\right)$, in some sufficiently small neighbourhood of $(\bar{p}, \bar{q})$, return to that neighbourhood after some time $t$ close to $\bar{T}$ and none of the trajectories ever strays far away from the periodic orbit during time $t$. Now, the action integral $S\left(q^{\prime \prime}, q^{\prime}, E\right)$ is taken along the trajectory that starts at $q^{\prime}$ and ends up at $q^{\prime \prime}$ after one, two or more turns while moving at energy $E$. Note that each energy $E$ has a full complement of periodic orbits and the action integral is a well-defined function of energy for each periodic orbit.

The displacements $\delta p=p-\bar{p}$ and $\delta q=q-\bar{q}$ are given by

$$
\begin{aligned}
\delta p^{\prime} & =-\frac{\partial^{2} S}{\partial q^{\prime} \partial q^{\prime}} \delta q^{\prime}-\frac{\partial^{2} S}{\partial q^{\prime} \partial q^{\prime \prime}} \delta q^{\prime \prime} \\
\delta p^{\prime \prime} & =-\frac{\partial^{2} S}{\partial q^{\prime \prime} \partial q^{\prime}} \delta q^{\prime}+\frac{\partial^{2} S}{\partial q^{\prime \prime} \partial q^{\prime \prime}} \delta q^{\prime \prime}
\end{aligned}
$$

or in abbreviated form

$$
\delta p^{\prime}=-a \delta q^{\prime}-b \delta q^{\prime \prime} ; \quad \delta p^{\prime \prime}=b^{\dagger} \delta q^{\prime}+c \delta q^{\prime \prime} .
$$

In order to understand the geometry of the trajectories in the neighbourhood of the periodic orbit, one would like to have formulas for $\delta p^{\prime \prime}$ and $\delta q^{\prime \prime}$ in terms of $\delta p^{\prime}$ and $\delta q^{\prime}$, such as

$$
\delta q^{\prime \prime}=A \delta q^{\prime}+B \delta p^{\prime} ; \quad \delta p^{\prime \prime}=C \delta q^{\prime}+D \delta p^{\prime} .
$$

with $A=-b^{-1} a, B=-b^{-1}, C=b^{\dagger}-c b^{-1} a$ and $D=-c b^{-1}$.

The behaviour of the trajectories are now described by the linear transformation $\left(\delta p^{\prime}, \delta q^{\prime}\right) \rightarrow\left(\delta p^{\prime \prime}, \delta q^{\prime \prime}\right)$ of $(2.18)$. The trajectories can now be classified according to the eigenvalues $\lambda$ of this transformation to be [Gu 90]

(a) elliptic for $\lambda=e^{ \pm i \chi}$ with $\chi$ real;

(b) direct parabolic for $\lambda=1$ or inverse parabolic for $\lambda=-1$;

(c) direct hyperbolic for $\lambda=e^{ \pm \chi}$, or inverse hyperbolic for $\lambda=-e^{ \pm \chi}$ with $\chi$ real;

(d) loxodromic for $\lambda=e^{ \pm u \pm i v}$ with independent signs and real values of $u$ and $v$. 
The situation in an integrable system can only be reconciled with the parabolic case so that integrable systems are nongeneric. If a mechanical system, with two degrees of freedom, is generic, the neighbourhood of a periodic orbit has to be elliptic (stable) or hyperbolic (unstable). In most cases there is an intimate mixture of elliptic and hyperbolic behaviour which is called soft chaos in the literature [Gu 90].

An alternative criterion for integrability is found in the multiperiodic nature of trajectories. The coordinates of a trajectory can be expanded in a Fourier series with as many frequencies as degrees of freedom. The power spectrum $I(\omega)$ of the autocorrelation function $r(\tau)$ can then be obtained (see §3.7). The power spectrum of an integrable system has sharp peaks in contrast to a nonintegrable system where broadened lines appear.

The power spectrum can now be used to obtain an accurate classical approximation to the quantal energy levels. By trying many initial conditions, one will eventually obtain a function $E\left(\omega_{1}, \omega_{2}, \ldots, \omega_{f}\right)$ which gives the the energy of the trajectory whose frequencies are found to be $\omega_{1}, \omega_{2}, \ldots, \omega_{f} . E(\omega)$ can now be transformed to $E_{i}$ which is the classical approximation to the quantal energy levels [Gu 90]. Tanner et al found the remaining periodic orbits in the strongly chaotic system of the hydrogen atom in a magnetic field. They then used this procedure to obtain accurate approximate quantum eigenvalues for this system [TS 91]. This is evidence of a connection between the periodic orbits and energy eigenvalues which will be indicated by Gutzwiller's trace formula discussed later.

Unlike the nearest neighbour spacing distribution there is a conjecture that the periodic orbit formalism is not the ideal probe to study the fine structure of the spectrum [Oz 88]. The fine structure is, however, governed by the exceptional points of the spectrum (see Chapter 4). The exceptional points also determine the form of the spectrum so that there must be a connection between the exceptional points and the remains of periodic orbits found in quantum chaotic systems (see discussion at end of $\S 2.4 .4$ ).

If an integrable system is perturbed strongly enough, the tori with periodic orbits will break up. The only surviving smooth invariant manifolds, beyond the energy shell itself, will then be isolated periodic orbits. The semiclassical limit of stationary states must therefore bear some relation to these invariant curves. The uncertainty principle, though, prevents us from attempting to tighten too closely the association between a given orbit and a specific state. As a last resort, a state has to rely on a collection of periodic orbits or at least on a periodic orbit "dressed" with the local motion around it [Oz 88].

The basic instrument for revealing the contribution of periodic orbits is the semiclassical propagator (Van Vleck's formula in (2.8)) for points that return to their initial positions. The propagator can also be represented as a sum over the intensities of the stationary wave functions. A local analysis establishes that individual wave intensities may exhibit strong 'scars' along some periodic orbits. The trace of the propagator gives the density of states as a sum over all periodic orbits. This is known 
as Gutzwiller's trace formula.

\subsubsection{Scarring of Eigenfunctions}

The state of a quantum mechanical system is uniquely described by its complexvalued eigenfunctions $\psi(\mathbf{q})$. The stationary states can be expected to display whatever chaotic features can show up in the framework of quantum mechanics. These features will, however, only show up in the highly excited states which brings us back to the limit of small Planck's constant, and thus to the connection with classical mechanics.

Heller studied the eigenstates of a two dimensional classical chaotic system e.g. the stadium billiard to obtain a detailed picture of the eigenstates [He 84]. In using Gaussian wave packets, he constructed the eigenfunctions so that a graph of $|\psi(x, y)|^{2}$ is feasible. When a certain threshold is exceeded, $|\psi|^{2}$ is plotted and the results are striking as shown in Fig. (2.2a).

From Fig. (2.2a) we conclude that some eigenstates has an intensity pattern showing scars i.e. narrow linear regions with an enhanced intensity which stands out clearly. The scars coalesce around the unstable periodic orbits ever more closely as $\hbar \rightarrow 0$. This is shown in Fig. (2.2b) which shows the major contributing periodic orbit in each case. From Gutzwiller's trace formula it is deduced that scarring is the localisation of the eigenfunctions around the least unstable classical periodic orbits (see $\S 2.4 .4$ ).

The intensity is obtained by noting that there is, usually, an infinity of periodic orbits, with arbitrary long periods, on the energy shell. The sum of the successive repetitions of a single periodic orbit supplies the total intensity of its scar. The intensity is thus determined by the parameter

$$
I \approx \frac{2 \pi}{\lambda \tau}
$$

with $\lambda$ the Lyaponov exponent and $\tau$ the period. A periodic orbit will show up in this manner provided it is not to unstable. The stability is given by

$$
\chi=\frac{2 \pi \lambda}{\omega}
$$

with $\omega$ the frequency of the periodic orbit. Periodic orbits are stable if $\chi$ is small and unstable if $\chi$ is large ( $\lambda$ large compared to $\omega$ ). Scars of periodic orbits will appear if $\chi<2 \pi$. The total contribution of an unstable periodic orbit is absolutely convergent and tends to zero as $\lambda \rightarrow \infty$. It is, therefore, the orbits with a small Lyaponov exponent that will have strong scars; but even in the limit $\lambda \rightarrow 0$ the intensities will have broad peaks denoting unstable periodic orbits that are not tied to a single eigenenergy. Between the peaks the total contribution of the scars is destructive, so that for these energies, the orbits contribute weak antiscars. 
Figure 2.2: (a) Scarred eigenstates of the stadium with (b) the corresponding classical unstable periodic orbits (from [He 84]). 


\subsubsection{The Gutzwiller Trace Formula}

The Gutzwiller trace formula connects a function of time or of energy, computed in quantum mechanics, to another such function calculated in classical mechanics. This equality is only valid in the limit of $\hbar \rightarrow 0$. The exact formal classical expressions depend on the behaviour of the classical dynamical system, whether regular, softly or harshly chaotic but the quantum expressions are independent of this classical predicament. The main idea is to use the knowledge of the classical behaviour, to compute the classical expressions, and then switch sides so as to draw conclusions about the quantum mechanics of the system. The trace formula relies on knowledge of the classical periodic orbits to understand the quantal spectrum or the quantal spectrum can be used to characterize the periodic orbits.

Van Vleck's formula (2.5) and the classical Green's function $G_{c}(2.10)$ are assumed to be valid approximations for chaotic Hamiltonian systems. The difficulty lies in finding all the classical trajectories from the starting point $q^{\prime}$ to the end point $q^{\prime \prime}$, either in the given time $t$ or at the given energy $E$, for a chaotic dynamical system. Up to now it was only possible to take the trace of $G_{c}$. Each term in the sum over classical periodic orbits is treated separately and remains separate in all further developments. There is no simple way to compare different trajectories and the presence of the sharp spikes in the density of states will remain a problem without a general solution.

Gutzwiller was able to carry out the sum over classical trajectories using Feynman's path integral in (2.15) as well as a phase space representation such as Wigner's function [HO 84]. In calculating the sum Gutzwiller obtained a semiclassical approximation $g_{c}(E)$ to the trace $g(E)$ of the quantal Green's function in (2.4) where [Gu 71]

$$
g(E)=\int d q^{f} G(q, q, E)=\sum_{j=0}^{\infty} \frac{1}{E-E_{j}}
$$

and

$$
g_{c}(E)=\operatorname{Tr} G_{c}\left(q^{\prime \prime}, q^{\prime}, E\right)=\frac{1}{i \hbar} \sum_{\text {p.o. }} \frac{T_{0}}{2 \sinh (\chi / 2)} \exp \left[i\left(\frac{S}{\hbar}-\beta \frac{\pi}{2}\right)\right]
$$

with $\chi$ defined in (2.19) and (2.21). Each term in the sum over periodic orbits consists of an amplitude $A=T_{0} /[2 i \hbar \sinh (\chi / 2)]$ and a phase factor with $\beta$ the Maslov index of each orbit. The primitive period $T_{0}$, the instability exponent $\chi$ and the action integral $S$ are continuous functions of the energy $E$ where the energy is assumed to be a complex number in the neighbourhood of the real axis [Bo 92]. The superposition of the smooth classical functions of $E$ is expected to yield at least an approximate spectrum for the quantum mechanical energy levels.

The quantal density of energy levels of a particular system is given by

$$
\rho(E)=\sum_{i} \delta\left(E-E_{i}\right)=-\frac{1}{\pi} \Im g(E) .
$$


which can be separated in a smooth and fluctuating part (see §3.4)

$$
\rho(E)=\bar{\rho}(E)+\rho_{\text {fluc }} .
$$

Semiclassically $\bar{\rho}$ is given by Weyl's rule in (3.8) and the fluctuating part by

$$
\rho_{\text {fluc }}=-\frac{1}{\pi} \Im g_{c}(E)
$$

such that

$$
\rho(E)=\bar{\rho}(E)+\Im\left\{\frac{i}{\pi \hbar} \sum_{r, j} A_{r, j} \exp \left[i j\left(\frac{S_{r}}{\hbar}-\beta_{r} \frac{\pi}{2}\right)\right]\right\} .
$$

Equation (2.25) is the density of states in terms of the trace formula. The label $r$ runs over all primitive orbits and $j=1,2,3, \ldots$ counts the number of traversals of the periodic orbit. For integrable two dimensional systems $A_{r, j}$ falls off as $j^{-1 / 2}$ for large $j$; for isolated stable orbits $A_{r, j}$ oscillates as a function of $j$ whereas it is exponentially damped in $j$ for isolated unstable orbits. This means that fulfillment of the condition in (2.2) for isolated unstable periodic orbits does not lead to sharp peaks, but only to smooth maxima in the fluctuating part of the level density. Therefore, the power spectrum of the fluctuating part of the level density of a chaotic system will show sharp peaks. Each peak can be associated with a classical periodic orbit and for each periodic orbit there is a corresponding peak in the Fourier transformed spectra. The locations of the peaks agree with the directly calculated scaled action of the classical orbits. The trace formula thus provides a simple but powerful way of, quantitatively, explaining quantum spectra in the low frequency domain (see §6.8) [Wi 87a], [FW 89].

The trace formula has been used by Bogomolny [Bo 92], Wintgen et al [Wi 87a], [WF 87], [WH 89] and Eckhard et al [EHP 89] to analyse the scars in the quantum eigenstates using the semiclassical level density given in (2.25). They show explicitly how the scars are associated with classical periodic orbits i.e. how the eigenstates localise around the isolated least unstable periodic orbits in the quantum system (also see $\S 5.8$ and $\S 6.8$ ). Rost et al studied the nodal structure of the wave functions of the Helium atom [RG 91] while Ezra et al calculated the energy of doubly excited states for Helium [ER 91].

Though of great theoretical interest, the trace formula in itself merely relates our ignorance of the density of states to the even more mysterious maze of periodic orbits. We can only attempt to explain the semiclassical fluctuations of the density of states by supplying further information about the distribution of periodic orbits [Oz 88]. This might be possible by obtaining a connection between the periodic orbits and the exceptional points. The distribution of exceptional points can, for certain systems, be obtained relatively easily (see Chapter 4) so that the necessary information on the distribution of periodic orbits are available. This important point is dealt with, in greater depth, in Chapter 6. 


\subsection{Experimental Evidence of Quantum Chaos}

The most profound experimental evidence of quantum chaos is shown in the hydrogen atom in a strong uniform magnetic field [HM 88] [MW 86]. The experimental spectrum has also been tested against the theoretical spectrum and found to be extremely accurate [WH 86]. The results, from a statistical analysis, thus show Gaussian Orthogonal Ensemble (GOE) fluctuations. By analysing the Fourier transform of the experimentally obtained spectrum, periodic orbits have been associated with the relevant peaks. These also coincide with theoretical calculations [WF 87].

GOE fluctuations have also been shown to occur in the spectra of $\mathrm{NO}_{2}$ [ZK 88] molecules and $\mathrm{Al}^{26}$ [MB 88]. The last example was subsequently explained by using a random matrix model involving the GOE [GW 90]. The theoretical spectrum of $\mathrm{NO}_{2}$ has been calculated by $\mathrm{Lu}$ et al and found to be accurate when compared with the experimental spectrum [LZ 91]. The hydrogen atom in a strong electric field can, theoretically, be associated with the periodically kicked rotor which is classically, and quantally, chaotic. The quantum kicked rotor shows dynamical localisation (which is globally similar to Anderson localisation) of the eigenfunctions and suppression of chaotic behaviour in contradiction to the classical behaviour [Fi 92], [Iz 90], [Ko 92]. The same features are observed experimentally for the microwave ionisation of highly excited hydrogen and Rubidium atoms [JS 91]. Experiments on disordered conductors (mesoscopic systems) show the localisation of the eigenfunctions and GOE fluctuations [Pi 92] which can be described using Random Matrix Theories [MF 91], [We 92]. Haake et al studied the spectrum of a microwave resonator, which corresponds to a pseudo-integrable billiard, which also shows GOE type fluctuations [HL 91]. Experiments with electrons in quantum wells are also proposed.

An astonishing confirmation of quantum chaos, and the semiclassical approach, was given by Stöckmann and Stein. They analysed the two dimensional stadium billiard which is known to be chaotic both classically and quantum mechanically [Oz 88], [Sc 84], [Za 85], [BG 84]. They first measured the spectrum experimentally and showed that it correlates with the theoretical prediction and thus shows GOE fluctuations [SS 90]. The wave functions were also measured experimentally which resemble the results of Heller (see $\S 2.4 .3$ ) exactly. The experimental results are shown in Fig. (2.3) (compare with Fig. (2.2)) [SS 92]. The first experimental observation of scarred eigenfunctions was actually given by Sridhar [Sr 91]. He studied microwave cavities shaped in the form of two dimensional Sinai billiards consisting of a circular disc inside a rectangle. The experimental and theoretical results are in good agreement and some unstable periodic orbits, associated to the scarred eigenfunctions, were obtained.

$æ$ 
Figure 2.3: Experimental eigenstates of the stadium with the corresponding classical periodic orbit. These eigenstates correspond to the theoretical states shown in Fig. (2.2) (from [SS 92]). 


\section{Chapter 3}

\section{Statistical Behaviour of Chaotic Systems}

The quantum mechanics of a dynamical system in a compact space yields a set of discrete energy levels rather than the continuum obtained in classical mechanics. Details of the spectrum change from system to system, but certain properties are quite general, and some seem to be directly correlated with the nature of the chaos in the corresponding classical system. These general properties of spectra are extracted and described through a thorough statistical analysis which shows striking similarities to results from Random Matrix Theories.

\subsection{Introduction}

The statistical analysis of the behaviour of a system gives useful information on the properties of the system. A remarkable property of universality has been established for the spectral fluctuations of quantum systems with classical chaotic counterparts [BGS 86]. The spectral fluctuations were found to depend only on general spacetime symmetry considerations and are the same as those of Random Matrix Ensembles [FMP 78]. In contrast, integrable systems do not display the same spectral fluctuation behaviour as chaotic systems. These systems give rise to mainly Poissonian fluctuations which are characterised by a complete absence of level correlations whereas random matrix spectra are typically very rigid with strong level correlations. Therefore by studying the level fluctuations of a quantum system we learn about the dynamics of the classical analogue or vice versa.

The richness of behaviour of quantum systems is not exhausted by their spectral properties but an enormous body of information is encoded in the eigenfunctions. Because of their multidimensionality, eigenfunctions are technically more difficult to study than the spectrum. Such an analysis focuses on concepts like scarring (described in the previous chapter) and localisation of eigenfunctions [He 84], [Iz 90]. 
In this chapter, a survey of the relevant statistical measures, used in the study of 'Quantum Chaos', are highlighted and briefly discussed. In the first part, Random Matrix Theories are introduced and their relevance to quantum chaos outlined. The second part describes the level repulsion in chaotic systems and the unfolding of spectra with the help of Weyl's rule. The third part features the level correlations in spectra and introduces the Nearest Neighbour Spacing Distribution and the $\bar{\Delta}_{3^{-}}$ statistic. The fourth part describes statistical functions taken from Time Series Analysis which are used to enhance our knowledge about the system itself. These functions include the autocorrelation coefficients and power spectrum. The last part presents the concept of localisation of eigenfunctions making use of the information entropy.

\subsection{Statistical Analysis}

It is often taken for granted that one resorts to statistical studies of certain systems because detailed properties of the spectrum are not really open to calculation. This is one of the reasons but not the main one. Such studies, whose nature is essentially different from the study of individual levels, are of interest because they reveal new features of the system, and they can be essential for a more complete understanding. Thirty years ago, Dyson made an eloquent resumé of the situation [Dy 62] (reprinted in [BG 84])

"Recent theoretical analyses have had impressive success in interpreting the detailed structure of the low-lying excited states of complex nuclei. Still there must be a point beyond which such analyses of individual levels cannot usefully go. It is improbable that level assignments based on shell structure and collective or individual particle quantum numbers can ever be pushed as far as the millionth level. It is therefore reasonable to inquire whether the highly excited states may be understood from the diametrically opposite point of view, assuming as working hypothesis that all shell structure is washed out and that no quantum numbers other than spin and parity remain good. The results of such an inquiry will be a statistical theory of energy levels. The statistical theory will not predict the detailed sequence of levels in any one nucleus, but it will describe the general appearance and the degree of irregularity of the level structure, that is expected to occur in any nucleus which is too complicated to be understood in detail.

In ordinary statistical mechanics a comparable renunciation of exact knowledge is made. By assuming all states of a very large ensemble to be equally probable, one obtains useful information about the overall behaviour of a complex system when the observation of the states in all its detail is impossible. This type of statistical mechanics is clearly inadequate for the 
discussion of nuclear energy levels. We wish to make statements about the fine detail of the level structure and such statements cannot be made in terms of an ensemble of states. What is required is a new kind of statistical mechanics in which we renounce exact knowledge, not of the state of a system, but of the nature of the system itself. We picture a complex nucleus as a 'black box' in which a large number of particles are interacting according to unknown laws. The problem is then to define in a mathematical precise way an ensemble of systems in which all possible laws of interaction are equally probable."

According to Dyson, in order to study a highly dense spectrum, an ensemble of systems must be defined. There is a strong logical expectation, though no rigorous mathematical proof, that an ensemble average will correctly describe the behaviour of one particular system which is under observation. The appropriate language to define such an ensemble is provided by Random Matrix Theories (RMT). With this theory the physical laws that govern the statistical behaviour of systems can be extracted.

\subsubsection{Random Matrix Theories}

Random Matrix Theories have their origin in the following physical problems:

- Hamiltonians of heavy nuclei are either unknown, or too complicated to be solved, and

- whilst most of the low energy states can be described, using appropriate nuclear models, the level density increases rapidly with the excitation energy $E$ such as

$$
\rho(E) \sim \exp (\sqrt{E}) .
$$

In the region of the neutron threshold $(E \sim 6 \mathrm{MeV})$, the number of levels is so high, and the intermixing so strong, that a detailed description of the individual levels is impossible. When the complications, however, increase beyond a certain point it is only necessary to explain the average properties of the levels which is much simpler.

For theoretical studies it is thus impossible to integrate the equations of motion and the introduction of ensembles of operators are essential. Through a Random Matrix Theory (RMT) analysis of systems [Bo 91]

1. simplicity may emerge in situations which initially appear as desperately complicated and random, and

2. probabilistic and statistical arguments may be fruitfully applied to systems where no external randomness nor stochasticity has been introduced from the outside. 
To avoid the difficulty of working with an infinite dimensional Hilbert space, we make approximations amounting to a truncation, keeping only the part of Hilbert space that is relevant to the problem at hand ${ }^{1}$. We are also only interested in the discrete part of the energy level schemes of various quantum systems so that we approximate the Hilbert space by one having a finite, though large, number of dimensions. By choosing a basis in this space, we represent the Hamiltonians by finite dimensional Hermitian matrices. The elements of these matrices are random variables whose distributions are restricted only by the general symmetry properties we might impose on the ensemble of operators. The eigenvalues and eigenfunctions of these ensembles are then studied statistically.

Wigner was the first to propose the hypothesis that the local statistical behaviour of levels in a simple sequence ${ }^{2}$ is identical with the behaviour of the eigenvalues of an ensemble of random matrices [Wi 51], [Me 91]. This discovery of the similarity of the level statistics of RMT and quantum Hamiltonians was striking because this agreement can not be due to a presumed random character of the matrix elements of the latter.

The following method is employed when studying RMT: one considers an ensemble of Hamiltonian matrices $H_{i}$ such that $H_{i}$ are $N \times N$ stochastic matrices i.e., all the individual matrix elements are independent random variables. According to Dyson a proper ensemble of random matrices should fulfill the following requirements [Dy 72] (revised by Bohigas and Giannoni [BG 75]):

- It should predict a global level-density in agreement with observations.

- It should predict a local statistical behaviour of energy levels also in agreement with observation.

- Its definition should be physically plausible and

- the mathematical problems involved should be tractable.

This means that with RMT there is no physical input a priori but, the statistical behaviour of real physical systems are simulated. The power of RMT have not only been demonstrated in describing the behaviour of nuclear systems, but also in describing the behaviour of mesoscopic systems [We 92], [IW 90], [MF 91] and a variety of mathematical model systems like quartic oscillators [Bo 92a].

Random matrix models might have quite different global properties, however, the astonishing thing about them is that local fluctuation properties are always the same and determined only by the overall symmetries of the system. This shows that the behaviour of ergodic systems is universal, i.e. the statistics do not depend on the fine details of the system [CGR 90].

\footnotetext{
${ }^{1}$ The effects of the truncation have to be taken into account.

${ }^{2} \mathrm{~A}$ simple sequence is one whose levels all have the same spin, parity and other strictly conserved quantities which result from the symmetry of the system e.g. the eigenvalues of a heavy nucleus like $U^{239}$.
} 


\subsubsection{Gaussian Orthogonal Ensembles}

The underlying space-time symmetries obeyed by the system put important restrictions on the admissible matrix ensembles. If the Hamiltonian is time-reversal invariant and invariant under rotations the Hamiltonian matrices can be chosen real symmetric; if $H$ has time-reversal, but no rotational symmetry the matrices can be chosen self-dual Hermitian and if $H$ has no time-reversal symmetry, the matrices can be chosen Hermitian. For a system of real symmetric matrices the Gaussian Orthogonal Ensemble (GOE) would be an appropriate choice [BG 84], [BF 81], [Me 91].

\section{Definition 3.1 Gaussian Orthogonal Ensemble}

The GOE is defined in the space $T_{1 G}$ of real symmetric matrices with time-reversal and rotational invariance with the following two requirements:

1. The ensemble is invariant under every transformation

$$
H^{\prime} \longrightarrow W^{T} H W
$$

of $T_{1 G}$ onto itself, where $W$ is any real orthogonal matrix.

2. The various elements $H_{i j}, i \leq j$, are statistically independent random variables which are Gaussian distributed.

The Gaussian Orthogonal Ensemble can describe the statistical properties of many-particle systems, in which all the particles interact simultaneously with each other, with astounding success. Such a system is believed to be a truly chaotic one. It is therefore extensively used in the study of nuclear and atomic spectra.

A GOE of matrices describes the statistical properties of nuclear spectra very successfully in the higher energy regions $(\geq 5-10 \mathrm{MeV})$ where the nuclear system is complex and there are many degrees of freedom. Investigations of the energy levels of neutral and ionized atoms in the rare-earth region shows good agreement between experiment and the GOE predictions [CG 83].

A GOE of matrices exhibit many features ascribed to chaotic systems [BF 81]. It is tendered that the relationship between time reversal invariant systems, whose classical analogues are chaotic, and the GOE is universal. Because of this, the results obtained from a statistical analysis of a GOE, will serve as a standard to which other ensembles would be compared in order to see whether they show any properties of chaotic behaviour.

\subsection{Level Repulsion in Spectra}

Regular (linear) systems like the harmonic oscillator have completely rigid discrete spectra. The whole spectrum is analytically known and uniformly distributed. If, 
Figure 3.1: Segments of complex spectra, each containing 50 levels and rescaled to the same spectrum span. The arrowheads mark pairs of levels with spacings smaller than one quarter of the average spacing $D$ (from [BF 81]).

however, the quantal spectrum of a classical nonlinear system is calculated, it is found that analytical techniques do not suffice and the analysis must be done numerically. The energy levels are also not uniformly distributed but fluctuations occur. With energy-level fluctuations, we mean the departure from uniformity in the spectrum. The problem is to find what systems give rise to GOE fluctuations and to find when deviations may be expected [SVZ 85].

In order to understand the behaviour of level sequences, having very different origins, the stochastic laws governing them have to be unraveled [BG 84]. A starting point for such a study would be to consider spectra of different systems. In Figure (3.1), six spectra are displayed, each containing 50 levels rescaled to the same spectrum span and each with the same $(J, \pi)$ i.e. total angular momentum and parity.

Although the spectra have very different origins, they are similar in their general nature, for example, the number of spacings much smaller than the average spacing is statistically the same for (a), (b) and (c). This has been made evident by marking all average spacings $D$ smaller than $D / 4$. The similarity of the spectra is made more obvious in Figures $(3.2 \mathrm{a}-\mathrm{c})$, which give histograms of the nearest neighbour spacings ${ }^{3}$ for each spectrum. From Figures $(3.2 \mathrm{a}-\mathrm{c})$ we observe that the three spectra in Figures $(3.1 \mathrm{a}-\mathrm{c})$ have the same nearest neighbour spacing distribution, thus exhibiting the same statistical nature, though the spectra themselves differ in the three examples. The continuous curve (Wigner's distribution) fits them quite well.

In the spectra of Figures $(3.1 \mathrm{a}-\mathrm{c})$ small (and large) spacings have small probability of occurrence. Figures $(3.1 \mathrm{~d})$ and $(3.2 \mathrm{e})$ show a Poisson spectrum where the

\footnotetext{
${ }^{3}$ For a description see $\S 3.5 .1$
} 
Figure 3.2: Nearest neighbour spacing histograms for the six cases of Figure (3.1), constructed by considering all the available levels instead of the 50 shown (from [BF 81]).

energy levels form a complete random sequence. The spacings distribution is then a Poisson type in which small spacings predominate. If we compare Figures $(3.2 \mathrm{a}-\mathrm{c})$ to Figures $(3.2 \mathrm{~d})$ and (e) we discern that the spectra in Figures $(3.1 \mathrm{a}-\mathrm{c})$ display level repulsion. The spectral fluctuations are governed by the level repulsions ${ }^{4}$ which were first discussed by Von Neumann and Wigner [VW 29].

Figure $(3.1 \mathrm{f})$ shows an equally spaced sequence of levels or the so-called picket fence $^{5}$ spectrum. Figures $(3.1 \mathrm{~d})$ and (f) represents two limiting cases: maximum randomness and no randomness at all, respectively. For the system, giving rise to spectrum (f), the correlations between the spacings are maximum and it shows perfect short and long range order. However, the Poisson spectrum shows no correlations between spacings. The knowledge of a stretch of the spectrum puts no restriction on the behaviour of the spectrum beyond the interval considered. In intermediate situations between Poisson and the picket fence the degree of spectral rigidity will depend on the nature and strength of the correlations between spacings.

Before any statistical test can be undertaken, the secular behaviour of the spectrum has to be removed. This will ensure that the statistical measures on different spectra can be compared directly.

\footnotetext{
${ }^{4} \mathrm{~A}$ detailed analytical discussion of this phenomenon is given in Chapter 4

${ }^{5}$ This spectrum is totally rigid
} 


\subsection{Unfolding of Spectra and Weyl's Rule}

The level fluctuations are the microscopic fluctuations of the level density. Experimental densities have of course a "secular" variation i.e. a long range variation with energy. The operation between secular variation and spectral fluctuations is emphasised by introducing a new energy variable which maps the secular density to a constant. Such a mapping is known as an unfolding (or rectifying) of the spectrum. It results in a sequence of energy levels that is invariant of the secular variation of different types of spectra. Spectra with very different origins, and average behaviours, can then be compared statistically.

Let the spectrum of a Hamiltonian operator $H$ be discrete such that

$$
\left\{E_{1} \leq E_{2} \leq \ldots \leq E_{d} \leq \ldots\right\}
$$

are the eigenvalues of the system. To get a visual impression of the overall regularity of the level series we may define a staircase function which represents the experimental level distribution graphically by a plot of $N(E)$ against $E$ where $N(E)$ is the number of levels having energy between zero and $E$ [DM 63]. A typical staircase is shown in Fig. (3.3 a). It is also customary to measure the average level spacing by drawing a line having the same average slope as the staircase representing the average or uniform behaviour of the spectrum. Fluctuations in the level series then appear as deviations of the staircase from the smooth line.

The staircase function $N(E)$ can thus be separated in a smooth part $N_{a v}(E)$ and a fluctuating part $N_{f l}(E)$ [BG 84], [Bo 91] such that

$$
N(E)=N_{a v}(E)+N_{f l}(E)
$$

where

$$
N(E)=\sum_{i} \Theta\left(E-E_{i}\right)=\sum_{E_{i} \leq E} 1=\operatorname{tr} \Theta(E-H)
$$

with $\Theta$ the unit step function and $H$ the Hamiltonian under consideration. The level density (density of states), $\rho(E)$, is given by the derivative

$$
\rho(E)=\frac{d N}{d E}=\sum_{i=1}^{\infty} \delta\left(E-E_{i}\right)=\operatorname{tr} \delta(E-H)
$$

The secular variation with energy is depicted by the smooth part $N_{a v}(E)$. To get rid of $N_{a v}(E)$, the spectrum is "unfolded" through the map $E \rightarrow x$ so that

$$
x_{i}=N_{a v}\left(E_{i}\right) ; \quad i=1,2, \ldots
$$

The effect of Eq. (3.4) is that the sequence $\left\{x_{i}\right\}$ has on the average a constant mean spacing (or a constant density) equal to unity, irrespective of the particular form of $N_{a v}(E)$. The spectral average is equal to the ensemble average after unfolding. The 
Figure 3.3: (a) A staircase function $N(E)$ and its appropriate smooth function $N_{a v}(E)$. (b) The resultant level fluctuations.

fluctuating part of $\left\{x_{i}\right\}$ corresponds to the departures from uniformity. As a function of $i$, the deviation of the $i$-th "level" $x_{i}$ from the average behaviour is given by

$$
N_{f l}=x_{i}-\left(i-\frac{1}{2}\right) ; \quad i=1,2, \ldots
$$

which will fluctuate around zero. Level fluctuations of a spectrum with level repulsion are shown in Fig. (3.3 b).

The simplest estimate of $N_{a v}(E)$, for a $d$-dimensional system, can be obtained by applying the semiclassical rule that each quantum state is associated with a phasespace volume $h^{d}$ with $h$ Planck's constant. One therefore has [Bo 91]

$$
N_{a v}(E) \simeq \frac{1}{\Gamma(d / 2+1)}\left(\frac{m}{2 \pi \hbar^{2}}\right)^{d / 2} \int_{U(\mathbf{q})<E}[E-U(\mathbf{q})]^{d / 2} d \mathbf{q}
$$

If the potential $U(\mathbf{q})$ is homogeneous of degree $\mu$,

$$
U(\alpha \mathbf{q})=\alpha^{\mu} U(\mathbf{q}) ; \alpha \in \mathcal{R}
$$

and

$$
N_{a v}(E) \approx\left(\frac{E^{1 / 2+1 / \mu}}{\hbar}\right)^{d} .
$$

If $V(E)$ is the volume of the classical phase space, (3.6) can be rewritten to give Weyl's rule [Oz 88]

$$
N_{a v}(E) \approx \frac{V(E)}{(2 \pi \hbar)^{2 d}}, \quad E \rightarrow \infty
$$

with

$$
V(E)=\int_{\mathcal{R}^{f}} \int_{\mathcal{R}^{f}} d \mathbf{p} d \mathbf{q} \Theta(E-H(\mathbf{p}, \mathbf{q}))
$$

where $\Theta$ runs from the ground state $E_{0}$ to $E \leq U(\mathbf{q})$ with $U$ the potential, $H$ the Hamiltonian and $f$ the number of degrees of freedom of the system. Weyl's rule is completely general and no distinction is made as to the dynamical nature of the 
system, whether integrable, ergodic or otherwise. Because Weyl's rule is insensitive to the nature of the classical motion, it is only the more subtle properties of the spectrum (fluctuations of the density about the mean) that may distinguish the dynamical nature of the corresponding classical motion. In the limit of large $E, N_{a v}(E)$ is the approximate number of states with energy less or equal to $E$ which is determined by the volume within the corresponding energy shell, measured in units of $(2 \pi \hbar)^{f}$. The average density of states is given by

$$
\bar{\rho}(E)=\frac{d N_{a v}}{d E}=\frac{1}{(2 \pi \hbar)^{f}} \int_{\mathcal{R}^{f}} \int_{\mathcal{R}^{f}} d \mathbf{p} d \mathbf{q} \delta(E-H(\mathbf{p}, \mathbf{q}))
$$

which is consistent with Liouville's theorem and the adiabatic principle. For a more elaborate description of this method see Appendix C.

\subsection{Level Correlations}

To study the statistical properties of sequences of eigenvalues one defines suitable functions such as level spacings or correlation and cluster functions, or a statistic such as the $\bar{\Delta}_{3}$ [Bo 91].

The $n$-level correlation and cluster functions are defined as follows: let

$$
x_{1}, x_{2}, x_{3}, \ldots, x_{N}
$$

be the positions of $N$ points on the real axis, with average density unity, and let

$$
P_{N}\left(x_{1}, \ldots, x_{N}\right) d x_{1} \ldots d x_{N}
$$

be the probability of having one point at $x_{1}$, another at $x_{2}, \ldots$, another at $x_{N}$ within each of the intervals $\left[x_{j}, x_{j}+d x_{j}\right]$. The density of states is then given by

$$
\rho(x)=N \int \cdots \int P_{N}\left(x, x_{2}, \ldots, x_{N}\right) d x_{2} \cdots d x_{N}
$$

and the statistical properties of the sequence $\left\{x_{i}\right\}$ are characterised by the set of $n$-level correlation functions $R_{n}\left(x_{1}, x_{2}, \ldots, x_{n}\right)$

$$
R_{n}\left(x_{1}, x_{2}, \ldots, x_{n}\right)=\frac{N !}{(N-n) !} \int d x_{n+1} \ldots d x_{N} P_{N}\left(x_{1}, x_{2}, \ldots, x_{N}\right) .
$$

$R_{n}$ is the probability density of finding a level around each member of $\left\{x_{i}\right\}$ where the positions of the other levels remain unobserved. $R_{1}$ will give the overall level density.

For practical purposes it is convenient to work with the $n$-level cluster functions $Y_{n}$ defined by

$$
Y_{n}\left(x_{1}, x_{2}, \ldots, x_{n}\right)=\sum_{G}(-)^{n-m}(m-1) ! \prod_{j=1}^{m} R_{G_{j}}\left(x_{t}, \text { with } t \text { in } G_{j}\right)
$$


with $G$ any division of the indices $[1,2, \ldots, n]$ into $m$ subgroups $\left[G_{1}, G_{2}, \ldots, G_{m}\right]$ [BG 84], [Bo 91], [Me 91]. The advantage of the cluster functions are that they vanish when any one (or several) of the separations $\left|x_{i}-x_{j}\right|$ becomes large in comparison to the local mean level spacing. The function $Y_{n}$ describes the correlation properties of a single cluster of $n$ levels, isolated from the more trivial effects of lower order correlations. In the following subsections, the correlation and cluster measures which are in common use are described.

\subsubsection{The Nearest Neighbour Spacing Distribution}

The nearest neighbour spacing distribution is a measure of the correlation between adjacent levels in spectra i.e. it is a short range level correlation measure.

In order to obtain this measure, the distances (spacings) between the spectral points $\left\{x_{i}\right\}$ (after unfolding) must be calculated

$$
S=x_{i+1}-x_{i}, \quad i=1,2,3, \ldots, N .
$$

Now, let $P(S) d S$ be the probability that a spacing $S$ lies between $S$ and $S+d S$. From Eq. (3.3) we know that after unfolding

$$
\langle S\rangle=\int P(S) S d S=1
$$

The fluctuations of an unfolded spectrum can be revealed by plotting the distribution $P(S)$ in the form of a histogram.

For a set of random numbers, arranged in ascending order, $P(S)=e^{-S}$. A spectrum with such a distribution will have a large probability of level clustering $(P(0)=1)$ and a small probability of level repulsion so that for intermediate values of $S, P(S) \ll 1$. This spacing distribution is termed Poissonic and can be seen in Fig. (3.2).

The distribution of a spectrum with level repulsion has the form of a Wigner surmise derived by Von Neumann and Wigner [VW 29]. For a GOE of matrices the distribution is of the form

$$
P(S)=\frac{\pi}{2} S e^{-\pi S^{2} / 4}
$$

Mehta and Gaudin [MG 60] derived the form for $N \rightarrow \infty$. A GOE of systems avoids level clustering because $P(0)=0$ and for intermediate values of $S, P(S)>0$. The levels are, however, still close to one another so that $P(S)$ vanishes for large $S$. This shows that level repulsion is a distinct feature of this distribution and of any chaotic system $^{6}$ (see Fig. (3.2)) [CCG 85].

For a totally rigid, picket fence, spectrum the distribution is simply a delta function at $S=1$ so that

$$
P(S)=\delta(S-1)
$$


Figure 3.4: The nearest neighbour spacing distribution for Poisson (dashed curve), Wigner (solid) and Picket Fence (dotted) distributions.

The distributions for the aforementioned cases are shown in Fig. (3.4).

Eq. (3.13) can be generalised to higher moments of this correlation function. Define the spacing between the $i$-th and $(i+k)$-th spectral point to be

$$
S_{k}=x_{i+k}-x_{i} ; \quad i=1,2, \ldots, N ; \quad 0<k \leq N .
$$

$P_{k}\left(S_{k}\right)$ is then the $k$-th moment of the distribution of spacings between energy levels and

$$
\sum_{k=0}^{\infty} S_{k}(x)=R_{2}(x)=1-Y_{2}(x)
$$

with $R_{2}$ and $Y_{2}$ the two point correlation and cluster functions, respectively, as defined above.

\subsubsection{The Brody Parameter}

The degree of level repulsion can be measured by the Brody parameter $\omega$ (also known as the repulsion parameter). This parameter fits $P(S)$ to the Wigner distribution with the only fitting parameter $\omega$. It is based on a generalization of the Wigner distribution given by Eq. (3.14) and may be written [Br 73]

$$
P_{\omega}(z)=A Z^{\omega} \exp \left(-\alpha z^{1+\omega}\right), \quad z=\frac{S}{D}
$$

with $D$ the average level spacing; $D$ is unity for an unfolded spectrum. The parameters $A$ and $\omega$ are found from the conditions that the averages of $z$ and the area under the curve must be unity. The resultant values are

$$
A=(1+\omega) \alpha
$$

\footnotetext{
${ }^{6}$ All symmetries must be reduced out and there should be no quantum number mixing.
} 
with

$$
\alpha=\left[\Gamma\left(\frac{2+\omega}{1+\omega}\right)\right]^{1+\omega} .
$$

Here $\Gamma$ is the Gamma function and the value of $\omega$ is found by fitting (3.17) to the data (or a theoretical distribution) by least squares. It is found that $\omega$ varies in the range $0 \leq \omega \leq 1$. The Brody parameter is zero for the uncorrelated spacings of a Poisson spectrum, unity for a Wigner type spectrum and $\omega=0.953$ for the GOE.

\subsubsection{The $\bar{\Delta}_{3}$-statistic}

The $\bar{\Delta}_{3}$-statistic measures the rigidity of the spectrum i.e., the departure from uniformity of an observed run of levels.

This measure is obtained by noting that, after unfolding, the staircase function described in $\S 3.4$ has an average that is a straight line. For a GOE of systems (or chaotic systems) the steps of the staircase will, however, not be of equal length. This leads to deviations between each step of the staircase and the averaged straight line. Dyson and Mehta thought it possible to construct a statistical measure from the deviations between the staircase and the best straight line fitting it. This measures the overall irregularity of the calculated level series and will show to what extent the results are in agreement with the pattern associated with the results obtained from the spectra of GOE Hamiltonians [DM 63]. It is convenient to take the observed energy interval as $[\zeta, \zeta+L]$, starting at $\zeta$ and of length $L$.

The problem is to analyse the deviation of the staircase graph

$$
y=N(x)
$$

from a suitable straight line

$$
y=A x+B
$$

over a suitable length $L$ of the staircase where $A$ and $B$ are constants to be determined. This can be achieved by fitting (3.19) to (3.18) by a least squares criterion. The meansquare deviation of the staircase from the averaged straight line is given by

$$
\Delta=\min _{A, B}\left\{\frac{1}{L} \int_{\zeta}^{\zeta+L}[N(x)-A x-B]^{2} d x\right\} .
$$

The quantity $\Delta$ measures the irregularity of the level series in an interval between $[\zeta, \zeta+L]$ and of length $L$. With $\Delta$ we study the long range correlations of the levels, and, consequently is meaningful only when the interval contains a sufficiently large number of levels.

There are three possible ways of fitting the straight line to the staircase, which give rise to three different statistics $\Delta_{1}, \Delta_{2}$ and $\Delta_{3}$. For $\Delta_{1}, B=0$ and for $\Delta_{2}$ the line is constrained to pass through the fixed point $y=N(\zeta)$. We only look at the $\Delta_{3}$-statistic in which case $A$ and $B$ may vary independently. 
By minimizing ${ }^{7}$ (3.20) with respect to $A$ and $B$, the following measure is obtained

$$
\Delta_{3}=\frac{1}{2 L} \int N^{2}(x) d x-\frac{1}{4 L^{2}}\left[\int N(x) d x\right]^{2}-\frac{3}{4 L^{4}}\left[\int N(x) x d x\right]^{2} .
$$

To rewrite (3.21) in a usable manner, use is made of the geometric interpretation of the integration over $N(x) . N(x)$ is a step function so that $\int N(x) d x$ is the evaluation of the area under the stepfunction curve. The unfolded eigenvalues are also ordered from lowest to largest such that $\left\{\zeta \leq x_{1} \leq x_{2} \leq \ldots \leq x_{n} \leq \zeta+L\right\}$ with $n$ the number of eigenvalues in this interval. If $x_{n+1}=\zeta+L$, Eq. (3.21) can be transformed to the following simple form

$$
\begin{aligned}
\Delta_{3}(\zeta, L)= & \frac{n^{2}}{16}-\frac{1}{L^{2}}\left[\sum_{k} \tilde{x}_{k}\right]^{2}+\frac{3 n}{2 L^{2}}\left[\sum_{k} \tilde{x}_{k}^{2}\right] \\
& -\frac{3}{L^{4}}\left[\sum_{k} \tilde{x}_{k}^{2}\right]^{2}+\frac{1}{2 L}\left[\sum_{k}(n+1-2 k) \tilde{x}_{k}\right]
\end{aligned}
$$

where

$$
\tilde{x}_{k}=x_{k}-\left(\zeta+\frac{L}{2}\right) .
$$

The long-range character of $\Delta_{3}$ is reflected in the fact that the summation goes from 1 to $n$ [BG 75].

A meaningful statistical measure is obtained by taking the mean of $\Delta_{3}$ denoted by $\bar{\Delta}_{3}$. The mean is calculated by taking many non-overlapping adjacent segments of length $L$ of the spectrum, compute $\Delta_{3}$ with (3.22) for each segment and by performing the average. For a Poisson spectrum one obtains

$$
\bar{\Delta}_{3}(L)=\frac{L}{15}
$$

and for a picket fence

$$
\bar{\Delta}_{3}(L)=\frac{1}{12}
$$

$\bar{\Delta}_{3}$ for the GOE was calculated by Haq, Pandey and Bohigas viz. [HPB 82]

$$
\bar{\Delta}_{3}^{G O E}(L)=\frac{2}{L^{4}} \int_{0}^{L}\left(L^{3}-2 L^{2} r+r^{3}\right) \Sigma^{2}(r) d r
$$

where

$$
\begin{aligned}
\Sigma^{2}(L)= & \frac{2}{\pi}\left\{\ln (2 \pi L)+\gamma+1+\frac{1}{2}[\operatorname{Si}(\pi L)]^{2}-\frac{1}{2} \pi \operatorname{Si}(\pi L)\right. \\
& \left.-\cos (2 \pi L)-\operatorname{Ci}(2 \pi L)+\pi^{2} L\left[1-\frac{2}{\pi} \operatorname{Si}(2 \pi L)\right]\right\} .
\end{aligned}
$$

\footnotetext{
${ }^{7}$ We seek the extremum i.e. the point where the variation of $A$ and $B$ vanishes simultaneously.
} 
Figure 3.5: $\bar{\Delta}_{3}$ for Poisson (dashed line), GOE (solid) and picket fence (dotted) spectra.

Here $\Sigma^{2}$ is the variance of the number $n$ of levels in a given interval of length $L$. Si and $\mathrm{Ci}$ are the sine and cosine integrals respectively. In terms of the cluster function

$$
\bar{\Delta}_{3}^{G O E}(L)=\frac{L}{15}-\frac{1}{15 L^{4}} \int_{0}^{L}(L-r)^{3}\left(2 L^{2}-9 L r-3 r^{2}\right) Y_{2}(r) d r
$$

which is a pure two point measure. All three cases are depicted in Fig. (3.5). The large $L$ asymptotic of $\bar{\Delta}_{3}$ is given as [DM 63]

$$
\bar{\Delta}_{3}(L) \sim \frac{1}{\pi^{2}} \ln L-0.007, \quad L \geq 15 .
$$

In summary we conclude that the departure of the average value of $\bar{\Delta}_{3}(L)$ from the linear increase with $L$ characteristic of the Poisson spectrum will give, in intermediate situations between Poisson and picket fence, information on the correlations between spacings and on the spectral rigidity or spectral stiffness. From this and (3.25) (which becomes essentially straight for large $L$ ) we conclude that a GOE and therefore a chaotic spectrum exhibits long range order.

\subsection{Spectral Analysis}

To complete a statistical study of spectra, use is made of concepts from the theory of Spectral Analysis, Time Series Analysis and Stochastic Theory [Pr 76], [OE 78], [Pa 65], [Ch 84].

A time series $X(t)$ is the result of a process that varies with a parameter $t, t \in \mathcal{R}$. $t$ is usually taken as time. In studying such series we ask the following questions:

1. Is there a certain periodicity in the series? 
2. Is the series a random series?

3. If a series is known for $t<a$, can it be predicted for values $t>a$ ?

Series which are not time dependent but which have $n$ elements and are characterised by a parameter $m$ such that $m \in\left\{m_{1}, m_{2}, \ldots, m_{n}\right\}$ where the 'distances' between elements $m_{i j}=m_{i}-m_{j}$ are constant, can be seen as a certain class of time series. Series of discrete eigenvalues, sets of eigenfunctions or amplitudes of eigenfunctions are all elements of this class of time series. The theory of Spectral Analysis can thus be applied to analyse such series. The autocorrelation coefficients give an estimate to the correlation between different levels of a specific time series whilst the spectral density function is a measure of the periodicity in a sequence of levels.

Through studying the time series of a certain process, a better comprehension of the process itself comes to the fore. A much better insight into the behaviour of quantum systems can be expected through such an analysis of the eigenvalues and eigenfunctions [HK 90b], [Iz 90], [Ec 88]. The concepts from this theory, relevant to the study of quantum chaos, are outlined in the following subsections. The spectral density function is subsequently used to detect the remaining classical motion in the quantum system. This is done by obtaining the classical periodic orbits from the quantum spectra where use is made of the Gutzwiller trace formula [Gu 90], [Oz 88].

\subsubsection{The Autocorrelation Coefficients}

Suppose we have a random sample of $N$ pairs of observations on $(X(t), Y(t))$ in which the pair of values $\left(x_{i}, y_{j}\right)$ occurs $N_{i, j}$ times $(i=1,2, \ldots k ; j=1,2, \ldots l)$, so that $\sum_{i=1}^{k} \sum_{j=1}^{l} N_{i, j}=N$. A measure of the relation between the elements of two time series $X(t)$ and $Y(t)$ is given by the autocorrelation coefficient.

The linear or Pearson correlation coefficient is defined by

$$
r(N)=\frac{\operatorname{cov}\{X(t), Y(t)\}}{\operatorname{var}\{X(t)\} \operatorname{var}\{Y(t)\}}
$$

with cov and var denoting the covariance and variance respectively. For discrete processes

$$
r(N)=\frac{\sum_{i=1}^{N}\left(x_{i}-\bar{x}_{i}\right)\left(y_{i}-\bar{y}_{i}\right)}{\sqrt{\sum_{i=1}^{N}\left(x_{i}-\bar{x}_{i}\right)^{2}} \sqrt{\sum_{i=1}^{N}\left(y_{i}-\bar{y}_{i}\right)^{2}}}
$$

or more conveniently

$$
r(N)=\frac{N \sum_{i=1}^{N} x_{i} y_{i}-\sum_{i=1}^{N} x_{i} \sum_{i=1}^{N} y_{i}}{\left\{\left[N \sum_{i=1}^{N} x_{i}^{2}-\left(\sum_{i=1}^{N} x_{i}\right)^{2}\right]\left[N \sum_{i=1}^{N} y_{i}^{2}-\left(\sum_{i=1}^{N} y_{i}\right)^{2}\right]\right\}^{1 / 2}}
$$


Figure 3.6: The autocorrelation coefficients for a GOE series.

with $N$ the number of points and

$$
\bar{x}_{i}=\frac{1}{N} \sum_{i=1}^{N} x_{i} \text { and } \bar{y}_{i}=\frac{1}{N} \sum_{i=1}^{N} y_{i} .
$$

If we put $y_{i}=x_{i+k}$ with $k=1,2, \ldots, n(1 \leq n \leq N)$ the autocorrelation coefficient will be a measure of the 'similarity' between a realisation of $X(t)$ and the same realisation shifted to the left by $k$ units. The correlation coefficient is then given by

$$
r_{k}(N)=\frac{N \sum_{i=1}^{N-k} x_{i} x_{i+k}-\sum_{i=1}^{N-k} x_{i} \sum_{i=1}^{N-k} x_{i+k}}{\sqrt{\left[N \sum_{i=1}^{N-k} x_{i}^{2}-\left(\sum_{i=1}^{N-k} x_{i}\right)^{2}\right]\left[N \sum_{i=1}^{N-k} x_{i+k}^{2}-\left(\sum_{i=1}^{N-k} x_{i+k}\right)^{2}\right]}} .
$$

As $k$ increases we would expect the correlation between $X(t)$ and $X(t+k)$ to decrease. The rate at which $r_{k}(N)$ decays to zero may be interpreted as a measure of the 'memory' of the process. For a harmonic series $r_{k}$ is a delta function at each value of $k$ i.e., perfect correlation between series points. For a Poissonic series $r_{k}$ is zero so that there are no correlations between levels and for a GOE of systems, $r_{k}$ decays exponentially for increasing $k$, showing long range correlation and order. This is shown in Fig. (3.6).

\subsubsection{The Spectral Density Function}

One of the main aspects of Time Series Analysis is the search for periodicity in a real time series $X(t)$. This is done with discrete Fourier Analysis because $X(t)$ is a discrete function.

To do this a function $G(\omega)$ is defined which is the Fourier transform of $X(t)$ so that we have the Fourier pair

$$
\begin{aligned}
G\left(\omega_{k}\right) & =\frac{1}{\pi} \sum_{t=0}^{\infty} X(t) \cos \left(\omega_{k} t\right) \\
X(t) & =\frac{1}{\pi} \sum_{k=0}^{\infty} G\left(\omega_{k}\right) \cos \left(\omega_{k} t\right)
\end{aligned}
$$


Figure 3.7: (a) Experimental spectrum for Hydrogen in a magnetic field. (b) The corresponding power spectrum with closed orbits correlated to the respective resonances (from [HM 88]; also see Chapter 6).

with $\omega_{k}$ the angular frequency such that $-\pi \leq \omega_{k} \leq \pi$.

The total energy used over the interval $(-\infty, \infty)$ is given by Parseval's relation for Fourier transforms e.g.

$$
\sum_{t=-\infty}^{\infty} X^{2}(t)=\sum_{k=-\infty}^{\infty}\left|G\left(\omega_{k}\right)\right|^{2}
$$

With

$$
f\left(\omega_{k}\right)=\left|G\left(\omega_{k}\right)\right|^{2}
$$

the term $f\left(\omega_{k}\right)$ is the contribution, to the total energy, of each component of $X(t)$ with frequency between $\omega_{k}$ and $\omega_{k}+d \omega_{k} . f\left(\omega_{k}\right)$ is called the spectral density function. A graph of $f\left(\omega_{k}\right)$ against $\omega_{k}$ is termed the Power Spectrum of the series $X(t)$.

A regular series will then give a power spectrum consisting of discrete lines (sharp peaks) for the corresponding frequencies, whereas a Poissonic (or totally random white noise) series will have a horizontal straight line (every frequency can appear with equal probability). A pure GOE series has a broad continuum in $f\left(\omega_{k}\right)$ while the power spectrum of a chaotic system contains a few pronounced peeks which can be correlated to the remaining unstable periodic orbits of the classical system (see $\S 2.4 .4$ and $\S 6.8$ ) [WF 87], [MWH 86]. The actual energy spectrum and corresponding power spectrum for a chaotic system is shown in Fig. (3.7). Also shown are some closed periodic orbits which can be correlated to the peaks (resonances) of the power spectrum. Contrary to the universality hypothesis of Wigner, there is thus a distinct difference between the behaviour of a GOE matrix and a chaotic system which is explained in $\S 3.8$.

There is a relation between the autocorrelation coefficients and the spectral density function such that

$$
f\left(\omega_{k}\right)=\frac{1}{2 \pi}+\frac{1}{\pi} \sum_{l=1}^{\infty} r_{l} \cos \left(\omega_{k} l\right)
$$


Figure 3.8: Some localised and delocalised eigenfunctions for the kicked rotor. The probability $w_{n}=\left|\phi_{n}\right|$ is drawn on the vertical axis with the number of unperturbed states $n$ on the horizontal axis. $l_{H}$ is the localisation length (from [Iz 90]).

The autocorrelation and spectral density functions are used to analyse the fluctuation patterns $\left(N_{f l}(E)\right.$ in $\left.(3.5)\right)$ that are obtained when the eigenvalues of a system are unfolded.

\subsection{Eigenfunction Statistics and Localisation}

Unlike spectral statistics, little is known about the structure of the eigenfunctions for quantum systems which are strongly chaotic in the classical regime. On the other hand, localisation of quantum wave packets in cases where classical mechanics predicts diffusive behaviour is a well known phenomenon in solid-state physics. The time-independent Hamiltonian of a particle in a random potential on a line has a pure point spectrum with eigenfunctions exponentially localised in space. This is known as Anderson localisation (see [Fi 92] and references therein).

By the localisation of eigenfunctions, we mean that a finite number of unperturbed states, less than $N$, are essentially occupied by each eigenfunction. This is depicted in Fig. (3.8) which shows some localised and some fully extended (delocalised) eigenstates. The eigenfunctions of a GOE matrix, however, are completely delocalised and the fluctuations of the components have a Gaussian distribution as predicted by RMT [Bo 92a]. The eigenfunctions of a fully chaotic system shows the same random character and delocalisation when the dimension $N \rightarrow \infty$ [Iz 90]. Localisation of eigenfunctions was, however, found in quantum chaotic systems like the kicked rotor where the classical system is strongly chaotic [Ca 88]. This localisation is related to Anderson localisation and strongly suppresses the chaotic behaviour in the quantum 
system compared to that found in the classical model [Fi 92].

The localisation length (see Fig. (3.8)) of the eigenfunctions can be obtained by making use of the standard definitions in statistical mechanics and information theory. Let $\phi_{k}$ be the $k$-th eigenvector of the system, $u_{k}=\Re \phi_{k}$ and $w_{k}=u_{k}^{2}$ so that $\sum_{k=1}^{N} w_{k}=1$. The information entropy is defined as follows

$$
\mathcal{H}_{N}\left(u_{1}, u_{2}, \ldots, u_{N}\right)=-\sum_{k=1}^{N} w_{k} \ln w_{k} .
$$

Here $\mathcal{H}_{N}$ is essentially the logarithm of the number of sites significantly populated by the given eigenstate $\phi(E)$. If all the sites were equally populated, then $\mathcal{H}_{N}$ would be $\ln (N)$. If, on the other hand, $u_{k}$ were exponentially localised around some site $k_{0}$ with localization length $1 \ll l \ll N$, then

$$
\mathcal{H}_{N}=1+\ln l+O(1 / l) \text {. }
$$

In general, the quantity $\exp \left(\mathcal{H}_{N}\right)$ is proportional to the effective number of nonzero components $u_{k}$ of the eigenfunctions, and can be taken as a measure of localisation so that

$$
l_{k}=\exp \left(\mathcal{H}_{N}^{(k)}\right)
$$

Here, $l_{k}$ is the effective number of components of $\phi_{k}$ with not too small a value. $l_{k}$ will be $N$ for a totally extended eigenfunction and unity for a totally localised eigenfunction. For a GOE we have

$$
\mathcal{H}_{N}^{\mathrm{GOE}}=\Psi\left(\frac{1}{2} N+1\right)-\Psi\left(\frac{3}{2}\right) \approx \ln (0.48 N)+O(1 / N)
$$

with $\Psi$ the Digamma function.

To take the chaotic structure of the eigenfunctions into account, the entropy localisation length $l_{H}$ is defined where

$$
l_{H}=\exp \left(\mathcal{H}_{N}-\mathcal{H}_{N}^{\mathrm{GOE}}\right) .
$$

$l_{H}$ is normalised so that a completely chaotic state has a value of unity. An $N$ dimensional system will lead to $N$ values for $l_{H}$ in (3.34). The distribution of the localisation lengths can then be calculated. The cases for a regular, chaotic and Poissonic system are shown in Fig. (3.9 i).

The distribution of the localisation lengths indicates that $l_{H}$ has large fluctuations from one eigenfunction to another. The normalised mean localisation length $l_{d}$ is therefore defined to be

$$
l_{d}=\left\langle l_{H}\right\rangle=\exp \left(\left\langle\mathcal{H}_{N}\right\rangle-\mathcal{H}_{N}^{\mathrm{GOE}}\right) .
$$

The averaging is done over all eigenvectors of the same matrix (or over an ensemble of similar matrices). With the definition in $(3.35), l_{d}$ will be zero for a Poissonic system 
Figure 3.9: (i) Distribution of localisation lengths for (a) regular, (b) chaotic and (c) Poissonic systems. (ii) Mean localisation length $l_{d}$ for the kicked rotor (From [Iz 90]).

and unity for a fully chaotic system ${ }^{8} . l_{d}$ for the kicked rotor is shown in Fig. (3.9 ii) and that for the quartic oscillator is obtained and shown in $§ 5.6 .2$. Fig. (3.9 ii) shows that the kicked rotor is nonchaotic for small values of the parameter $k$ and becomes increasingly chaotic as $k$ increases.

A question that needs to be answered deals with the chosen basis in position space when we study the structure of the eigenfunctions. There is a basis in which all the eigenfunctions of the system are completely localised, namely, the basis where the Hamiltonian is diagonal. However, the probability in having such a basis in a physical setting in $N$-dimensional Hilbert space is negligibly small. It is thus very unlikely to have such a special basis from the outset and these special bases can be seen as exceptional ones. Still, the choice of the basis has to be kept in mind when studying the eigenfunctions of physical systems.

It should be noted that the random character of the eigenfunctions of a chaotic system, in position space, seems to contradict the scars as studied by Heller (see $\S 2.4 .3$ ). These scars appear for some eigenfunctions in the coherent state basis (or Wigner representation) in the form of increased density in the regions of phase space where unstable periodic trajectories of low periods are situated [He 84]. The structure of the eigenfunctions in the coherent state basis can thus not be treated as random since the regions of increased density are well predicted by the periodic orbits of the classical system. There is thus no contradiction and an interesting question is whether the scars can be detected in the distribution of $w_{n}$ ?

\footnotetext{
${ }^{8}$ In literature this is also denoted as hard chaos.
} 


\subsection{Breaking of the Universality Regime}

Bohigas, Giononni and Schmidt elaborated on the hypothesis of Wigner (see §3.2.1) and stated that the spectral fluctuations of systems, that are chaotic classically, are similar to GOE fluctuations. This relationship holds universally [BGS 84]. However, distinct differences are observed when comparing the fluctuating part, $N_{f l}$, of the spectrum of a system that is chaotic classically and that obtained from a pure GOE matrix. Chaotic systems like the Laplacian on a compact smooth surface of negative curvature [Gu 90]; the anisotropic Kepler problem [WM 88]; the zeros of the Riemann $\zeta$ function [Be 85]; the Hydrogen atom in a strong magnetic field [Wi 87a] and the quartic oscillator [ZM 86] all show such departures.

The eigenvalues of a chaotic system can show periodic components which are not visible in the spectrum of the random matrix. This is clearly seen when the power spectrum of a GOE matrix and that of a chaotic system is compared. The power spectrum of a quantum chaotic system can have distinct peaks which can be identified with the periods of the short periodic orbits whose instability is relatively weak (see Fig. (3.7)). These orbits are the classical orbits still remaining in the quantum system. A GOE of matrices only simulates a quantum chaotic system, it has therefore no relevance to an actual system and will thus not show the existence of any orbits.

Berry also pointed out that systematic deviations from RMT predictions for the $\bar{\Delta}_{3}$-statistic can occur and were due to the existence of classical periodic orbits [Be 85]. Berry argued that there are two scales in any problem. The first is the inner scale provided by the mean level spacing $N_{a v}$ where

$$
N_{a v}=1 / \bar{\rho} \sim h^{f}
$$

(see (3.6) and (3.9)) where $h$ is Planck's constant and $f$ the number of degrees of freedom. The second is the outer scale provided by $h$. All discussions so far concerned the inner scale [Bo 91]. The spectral properties with inner-scale resolution are determined by the very long classical periodic orbits whereas the ones with outer-scale resolution are determined by the short ones. The properties of the latter depend on each particular system and give rise to the nonuniversal features of the spectral fluctuations, features which are not contained in RMT descriptions. In investigating the zeros of the Riemann $\zeta$-function, Berry used the Gutzwiller trace formula to explain this nonuniversality and found excellent agreement between the measured number variance and his semiclassical description [Be 85], [Bo 92]. Wintgen studied the hydrogen atom in a strong magnetic field and also found the deviations between the measured $\bar{\Delta}_{3}$-statistic and that of the GOE to be due to the classical periodic orbits which can be accounted for by a semiclassical analysis [Wi 87a]. This is taken as evidence that the fact that the statistical behaviour of a quantum chaotic system follows the GOE behaviour is just a fluke of nature. Random Matrix Theories can therefore not lead to 
a conclusive description of the origin of the behaviour. The origin of quantum chaotic behaviour should be sought in the exceptional points discussed in the next chapter.

The Gutzwiller trace formula has thus been very successful in predicting spectral fluctuations at the outer scale energy resolution. Problems still remain to achieve inner-scale resolution. As stated in the last paragraph of $\$ 2.4 .4$, more information on the distribution of the periodic orbits is necessary to be able to obtain such a description. Ozorio de Almeide even stated that the periodic orbit formalism may not be the ideal probe for the study of such fine scales of the spectrum [Oz 88]. A promising method for investigating the spectrum at very fine scales is by studying the exceptional points. The exceptional point approach might be the bridge between the semiclassical treatment and the RMT description at the inner-scale. It is the aim of this thesis to build that bridge and the subsequent chapters deal with this problem in detail.

\subsection{Summary}

All the measures described in this chapter serve to analyse the spectrum and eigenfunctions of chaotic systems. A thorough statistical analysis of any spectral series and eigenfunctions will lead to a better understanding of the quantal system under consideration.

A comparison between the statistical results from a GOE of matrices and that of quantum chaotic systems led to the conjecture that GOE statistics are universal for systems with classical chaotic counterparts. Departures from this universality were, however, found in several systems. The departures from GOE is due to the fact that the GOE results only mimics the level fluctuations but it does not provide any information on the origin of the behaviour of the system. On the outer-scale (see §3.8) these deviations are thoroughly described with Gutzwiller's semiclassical analysis. A description of the spectrum on the very fine scale is not yet viable by the semiclassical formalism. An understanding is, however, amenable by investigating the exceptional points of the spectrum; this is outlined in the following chapters.

$æ$ 


\section{Chapter 4}

\section{Analytic Properties of the Spectrum}

Avoided level crossings play an important role in quantum mechanics such as nuclear physics, quantum electrodynamics and quantum chromodynamics [SW 72], [GH 86]. A distinct feature of a quantum chaotic system is the many avoided level crossings evident in the spectrum. The universal spectral behaviour of quantum chaotic systems (as was shown in Chapter 3) is directly linked to these avoided level crossings.

In this chapter the properties of the spectrum of a matrix representation of a quantum chaotic system, pertaining to a Hamiltonian $H(\lambda)=H_{0}+\lambda H_{1}$, are analysed analytically. All work is done in an irreducible subspace so that actual degeneracies of the selfadjoint problem can be excluded. The repulsion of energy levels governs all the spectral fluctuations. In turn, they are associated with the exceptional points of the operator. Exceptional points are a distinct property of the Hamilton operator. From this we note that if Hamiltonians in quantum mechanics are distinct from others by their spectral behaviour, such properties must reside with the intrinsic operator properties of the Hamiltonian.

The exceptional points determine the specific shape of the spectrum. An analysis of the distribution of these points will shed light on the intricate statistical behaviour of the spectrum. It is also conjectured that the scars of periodic orbits can be obtained from an exceptional points analysis. The exceptional point method thus provides a link between a semiclassical and spectral analysis.

\subsection{Introduction}

The approach used in this thesis is to investigate quantum chaos from the following point of view: if a matrix representation of a Hamiltonian, which is the quantised version of a classical chaotic system, is given, what is the mathematical mechanism that yields the special features of the spectrum within the particular range of the 
parameter where classical chaos is discerned? The answer lies in what is known as the exceptional points of an operator [ $\mathrm{Ka} 66]$.

The first part of this chapter describes the form of the Hamiltonians used throughout this thesis. The second part introduces the concept of avoided level crossings and describes how level repulsion comes about in a spectrum. The third part defines the exceptional points of a spectrum and shows how these points effect the form of the spectrum. The fourth part defines the line spectrum of a system and shows how the distribution of the exceptional points can be obtained confirming the statement that a high level density is a necessary condition for chaotic behaviour [HK 91]. To illuminate this point and to show the influence on the statistical behaviour of the eigenvalues and eigenvectors, the last part introduces two simple (though relevant) model Hamiltonians as demonstration.

\subsection{The Form of the Hamiltonian}

To elucidate the concept of exceptional points and to study quantum chaos, we investigate matrix Hamiltonian systems of the form

$$
H=H_{0}+\lambda H_{1}
$$

with $H_{0}$ and $H_{1}$ Hermitian matrices in Hilbert space $\ell_{2}(N)$. The parameter $\lambda$ can play the role of a perturbation parameter; it may serve to effect a phase transition or it may steer the system, under variation, from an ordered into a chaotic regime. It is assumed that the eigenvalues of both $H_{0}$ and $H_{1}$ are readily available and that $H_{0}$ is the matrix representation of a classical integrable system.

A plot of the energy levels $E_{k}(\lambda)$ pertaining to $H$, against the strength parameter $\lambda$, will show multiple isolated degeneracies if the system is regular and near degeneracies if the system is chaotic. If the symmetries relating to $H_{0}$ and $H_{1}$ are incompatible or simply if the two operators do not commute, the mixed problem is usually characterised by many avoided level crossings. This will lead to a region where the level statistics may, or may not, display the characteristics of quantum chaos. The precise patterns, such as the energy ranges for which the GOE statistics are established at a particular $\lambda$ value vary strongly from case to case - a general description is not at hand.

From the viewpoint followed in this thesis a representation where $H_{0}$ is a diagonal matrix will always be used. If a suitable analytic basis is not available, an orthogonal transformation is carried out such that

$$
H=D_{0}+\lambda U \cdot D_{1} \cdot U^{-1}
$$

with $D_{0}$ and $D_{1}$ the diagonal representations of $H_{0}$ and $H_{1}$ respectively. $U$ is an orthogonal matrix that diagonalises $H_{1}$ in the basis of diagonal $H_{0}$. Such a representation will facilitate the analysis in obtaining a suitable method for the calculation of the distribution of exceptional points as described in $\S 4.5$. 
Figure 4.1: Two sheets representing energy levels as functions of the parameters $\lambda$ and $\kappa$ with a degeneracy $P$ at $\lambda^{*}, \kappa^{*}$ and energy $E^{*}$. $A$ is a path missing the degeneracy and $B$ is a path enclosing it (from [Be 81]).

\subsection{Avoided Level Crossings}

In Chapter 3 it was shown that a universal feature of quantum chaotic systems is the many near degeneracies in their spectra. Because we work in a subspace where all degeneracies have been reduced out, actual degeneracies can not occur. This theorem was proven by Von Neumann and Wigner [VW 29] and states:

In order to produce level degeneracies in a generic real Hamiltonian system without symmetry, not one, but two parameters must be varied.

It can be stated differently: among all selfadjoint Hermitian matrices, those with a degeneracy have co-dimension three. When the system has time-reversal invariance, it has co-dimension two and no real degeneracies can occur.

To illustrate this theorem, let the system depend on two parameters $\lambda$ and $\kappa$ such that both can be varied. The two degenerate levels correspond to two sheets of an elliptic cone in the $(\lambda, \kappa, E)$ space near the degeneracy which occurs at a point $P=\left(\lambda^{*}, \kappa^{*}, E^{*}\right)$ called a diabolical point. This is shown in Fig. (4.1) [Be 81].

If only one parameter is varied ( $\lambda$ for instance) this corresponds to traversing a path in $(\lambda, \kappa)$-space that misses $\left(\lambda^{*}, \kappa^{*}\right)$ so that the energy level curves are hyperbolas cutting the cone. It seems as though the levels repel each other - they move closer together and then suddenly bounce away from one another as shown in Fig. (4.2). The wave functions, however, interchange their association (with an accompanying sign change) at the near degeneracy at $\lambda_{0}$ : if $\phi_{k}$ and $\phi_{m}$ are associated with levels $E_{k}$ and $E_{m}$ respectively for $\lambda<\lambda_{0}$ (see Fig. (4.2)), $\phi_{k}$ would be associated with $E_{m}$ and 
Figure 4.2: A near degeneracy at interaction strength $\lambda_{0}$. Although the levels do not cross, their corresponding wave functions interchange association at $\lambda_{0}$ (from [Be 81]).

$\phi_{m}$ with $E_{k}$ for $\lambda>\lambda_{0}$ [HK 90b]. The following question naturally arises: how do we know when the point $\left(\lambda^{*}, \kappa^{*}\right)$ on Fig. (4.1) is a true degeneracy rather than a very close approach of the two separate eigenvalue surfaces? The answer is that moving on a small circuit $B$ in $(\lambda, \kappa)$-space, surrounding $\left(\lambda^{*}, \kappa^{*}\right)$, each of the two wave functions changes sign if $\left(\lambda^{*}, \kappa^{*}\right)$ is a degeneracy of two levels, and the wave functions do not change sign if $\left(\lambda^{*}, \kappa^{*}\right)$ is not a degeneracy.

The phenomenon of "repulsion of levels" is very different from what happens in separable and integrable systems. In these cases a degeneracy typically occurs when just one parameter $\lambda$ is varied which can be seen by considering a separable two dimensional system [Be 81]. The energy levels are labelled with two quantum numbers $n$ and $m$

$$
E=E(m, n ; \lambda)
$$

For a given quantum state $\left(m^{*}, n^{*}\right)$, in the $(m, n)$ phase space, the energy corresponds to a contour of (4.3) that passes through $\left(m^{*}, n^{*}\right)$. If this contour does not pass through any other states, $\left(m^{*}, n^{*}\right)$ is nondegenerate. When, however, $\lambda$ is varied the slope of (4.3) changes so that the contour crosses other states producing "one-parameter" degeneracies. Such a degeneracy occurs near a point called an exceptional point and not a diabolical point as indicated above. Diabolical points refer to selfadjoint operators whereas exceptional points do not.

All the systems that we discuss can be represented by Hermitian matrices with time-reversal invariance and are functions of one parameter only $(\lambda$ in (4.2)). We thus have exceptional points and not diabolical points in the complex energy plane. There should not be any actual degeneracies in the spectrum because we work in the irreducible subspace of the system. Accidental degeneracies can, however, occur and the studied range of the spectrum has to be investigated to see whether this is the case.

From the discussion above we see that diabolical points are points in the real $(\lambda, \kappa)$ plane where degeneracies occur. When the system only depends on one parameter 
$\lambda$, there are no real values of $\lambda$ for which the eigenvalues coalesce. Two eigenvalues can, however, still coalesce in the complex $E$-plane corresponding to complex values of $\lambda$. The complex $\lambda$-plane thus contains important information on the behaviour of a system. By analysing the complex $\lambda$-plane a connection between the operators and the spectral fluctuations is obtained. For such an investigation we now define the concept of exceptional points.

\subsection{Exceptional Points}

The definition of exceptional points is general and applies also to operators in an infinite dimensional space, even when the spectrum of the operator has a continuum part [Ka 66], [Sh 88]. From the viewpoint taken in this thesis, we restrict ourselves to finite dimensional matrices $H_{0}$ and $H_{1}$ as in this case the role of the exceptional points and the associated Riemann sheet structure is thoroughly understood [HS 90]. We do not believe that restriction to matrices has a major impact on our conclusions since virtually all the practical work, even in connection with quantum chaos, is done in a truncated finite dimensional matrix space.

The physical significance of the exceptional points is due to their relation with avoided level crossings for real $\lambda$-values. The spectrum $E_{k}(\lambda), k=1, \ldots, N$ has branch point singularities at the exceptional points, in fact, any two of the $N$ levels are connected via a square root branch point [BHS 74]. If this happens near to the real $\lambda$-axis, a level repulsion will occur for the two levels for real $\lambda$-values. There is an analogy to the more widely known connection between the pole singularities of the scattering function and the resonance structure (shape) of the cross section: in a similar way as the positions of the poles, including their statistical properties, determine the measurable cross section, the exceptional points determine the shape of the spectrum and in particular the occurrences of avoided level crossings. This comparison must, however, be taken with care because the exceptional points are square root branch points and thus produce a Riemann sheet structure [HS 91a]. The distribution of the exceptional points will therefore determine the fluctuation properties of level spacings.

We exclude genuine degeneracies of the selfadjoint problem ${ }^{1}$. The exceptional points then occur in the complex $\lambda$-plane. The positions of the exceptional points are fixed and are determined solely by $H_{0}$ and $H_{1}$. For large matrices it is prohibitive to determine the positions of the exceptional points. However, is it possible to determine the distribution reasonably well from knowledge of the two operators alone. This is shown in the following sections.

\footnotetext{
${ }^{1}$ Note that the operator is not selfadjoint for complex $\lambda$-values.
} 


\subsubsection{Definition of Exceptional points}

Let the Hamiltonian $H$ have the form given in (4.1) or (4.2). The eigenvalues $E_{n}(\lambda)$ of $H(\lambda)$ are the roots of the characteristic equation

$$
\operatorname{det}[E-H(\lambda)]=0 .
$$

The exceptional points of a Hamiltonian are defined as the complex values of $\lambda$ for which two different eigenvalues coincide [BW 69], [ZVW 83], [Ka 66]. To ensure that the root in (4.4) is a double zero, the fulfillment of an additional simultaneous equation

$$
\frac{d}{d E} \operatorname{det}[E-H(\lambda)]=0
$$

is necessary. Equations (4.4) and (4.5) are two polynomials of order $N$ and $(N-1)$, both in $E$ and $\lambda$, respectively. Their simultaneous solutions will give rise to $N(N-1)$ crossing points of the energy trajectories in the complex $E$-plane. These correspond to the exceptional points found in the complex $\lambda$-plane and they occur in $N(N-1) / 2$ complex conjugate pairs because (4.4) and (4.5) have real coefficients [HS 90].

If we assume that $H(\lambda)$ is irreducible with respect to symmetries, fulfillment of the two equations (4.4) and (4.5) simultaneously, is generically excluded for real $\lambda$ as this implies a genuine degeneracy of the two levels (see $\S(4.3)$ ). When solving (4.4) and (4.5) the real and imaginary parts are taken separately resulting in four real equations for four real solutions $\Re E, \Im E, \Re \lambda$ and $\Im \lambda$ with the latter two giving the positions of the exceptional points. A coalescence of the two roots of (4.4), as enforced by (4.5), will not allow an additional condition. We exclude coalescence of three roots of (4.4), in other words, generically, it is expected that

$$
\frac{d^{2}}{d E^{2}} \operatorname{det}[E-H(\lambda)] \neq 0
$$

and

$$
\frac{d}{d \lambda} \operatorname{det}[E-H(\lambda)] \neq 0
$$

at the points where (4.4) and (4.5) are obeyed. Consequently the polynomial

$$
\prod_{k=1}^{N}\left[E-E_{k}(\lambda)\right]=\operatorname{det}[E-H(\lambda)]
$$

vanishes linearly in $\lambda$ at the branch points [HS 91a]. A more complicated branch point of $E(\lambda)$ would require (4.4) to have a multiple root which is viewed as an artificial and nongeneric situation.

When a level $E_{k}$ shows level repulsion with a level $E_{k \pm 1}$, the roots of (4.4) coalesce in the complex plane viz.

$$
E_{k}\left(\lambda_{c}\right)=E_{k \pm 1}\left(\lambda_{c}\right) .
$$


The coefficients of Eq. (4.4) are real for real $\lambda$ and therefore

$$
E_{k}\left(\lambda_{c}\right)=\bar{E}_{k}\left(\bar{\lambda}_{c}\right)
$$

An alternative method for finding the exceptional points is by eliminating $E$ in (4.4) and (4.5). This leads to a new equation in $\lambda$ only where the roots of the discriminant are the exceptional points. Use is made of the following theorem [Co 74]: Let $f(x, y)$ and $h(x, y)$ be two polynomials such that

$$
\begin{aligned}
& f(x, y)=a_{0}(y) x^{n}+a_{1}(y) x^{n-1}+\ldots+a_{n}(y) \quad a_{0} \neq 0 \\
& h(x, y)=b_{0}(y) x^{m}+b_{1}(y) x^{m-1}+\ldots+b_{m}(y) ; \quad b_{0} \neq 0 .
\end{aligned}
$$

With certain linear combinations of these equations, the degree in $x$ can be reduced such that a new equation $g(y)$, which is only a function of $y$, is found. The degree in $y$ of $g(y)$ is $n(n-1)$. If however, $h=f^{\prime}=d f / d x, g(y)$ is the discriminant of $f(x, y)$ and $g(y)=0$ will lead to $n(n-1)$ common factors of $f$ and $f^{\prime}$.

From this theorem, the zeros of the discriminant of (4.4) will then result in the exceptional points of $H(\lambda)$. The solutions of the discriminant come in $N(N-1) / 2$ complex conjugate pairs and are denoted by $\lambda_{c}$ and $\bar{\lambda}_{c}$.

Equations (4.4) and (4.5) can analytically be solved only for $N=2,3$. Numerically it is very time consuming for large values of $N$ because the number of singularities to be searched for is $N(N-1) / 2$. On a main frame computer $N \leq 30$ was experimentally found to be practical [HS 91a] and the algorithms used found the exceptional points accurately using quadrupole precision. Problems also arise because the numerical procedures usually need guess values for all four quantities $\Re \lambda_{c}, \Im \lambda_{c}, \Re E$ and $\Im E$ as input. In our situations we work in infinite dimensional Hilbert space which we truncate for practical purposes. We thus need $N$ as large as possible and the methods described above are impractical. To overcome these problems, $\S 4.5$ describes a method for determining the distribution of exceptional points approximately. This procedure is possible for values of $N$ up to 3000 on a minisuper computer.

\subsubsection{Riemann Sheet Structure}

Equation (4.8) implies that if levels $E_{k}(\lambda)$ and $E_{m}(\lambda)$ are connected at the complex value $\lambda_{c}$ (exceptional point) and assume there the complex value $E_{c}$, they must have the form

$$
\begin{aligned}
& E_{k}(\lambda)=E_{c}+\sum_{t=1}^{\infty} d_{t}\left(+\sqrt{\lambda-\lambda_{c}}\right)^{t} \\
& E_{m}(\lambda)=E_{c}+\sum_{t=1}^{\infty} d_{t}\left(-\sqrt{\lambda-\lambda_{c}}\right)^{t}
\end{aligned}
$$

for a particular $m=k \pm 1$. Similar expansions exist about $\bar{\lambda}_{c}$. Only in this way can the determinant have a simple zero in $\lambda$ and a double zero in $E$ and be regular in $E$ 
and $\lambda$. These expansions have a finite radius of convergence determined by the next nearest singularity and the two eigenvalues $E_{k}$ and $E_{m}$ are connected by square root branch points at $\lambda=\lambda_{c}$ and $\lambda=\bar{\lambda}_{c}$.

The expansions in (4.11) indicate that $E_{k}$ and $E_{m}$ are the same analytic function on two different Riemann sheets [HS 91a]. Since $N(N-1) / 2$ complex conjugate pairs of exceptional points connect, analytically, all possible pairs of eigenvalues $E_{k}$ and $E_{m}$ $(k \neq m)$ in a similar way, generically the $N$ eigenvalues are the values of one analytic function on $N$ different Riemann sheets (for an in detail description see [Sa 91]).

\subsubsection{Parametric Dependence of Exceptional Points and the Line Spectrum}

The previous discussion can be illuminated by considering a two dimensional matrix problem where $D_{0}$ is diagonal with eigenvalues $\epsilon_{1}$ and $\epsilon_{2}$, while $H_{1}$ is represented in the form $H_{1}=U \cdot D_{1} \cdot U^{-1}$ as given in (4.2). Here, the diagonal matrix $D_{1}$ contains the eigenvalues $\omega_{1}$ and $\omega_{2}$ of the matrix $H_{1}$. $U$ can be represented by the rotation

$$
U=\left(\begin{array}{cc}
\cos \varphi & -\sin \varphi \\
\sin \varphi & \cos \varphi
\end{array}\right)
$$

where the angle $\varphi$ is determined by the eigenvector matrix $U$ obtained by diagonalising $H_{1}$.

The eigenvalues of the problem $D_{0}+\lambda H_{1}$ are

$$
E_{1,2}(\lambda)=\frac{\epsilon_{1}+\epsilon_{2}+\lambda\left(\omega_{1}+\omega_{2}\right)}{2} \pm S
$$

where

$$
S=\left\{\left(\frac{\epsilon_{1}-\epsilon_{2}}{2}\right)^{2}+\left(\frac{\lambda\left(\omega_{1}-\omega_{2}\right)}{2}\right)^{2}+\frac{1}{2} \lambda\left(\epsilon_{1}-\epsilon_{2}\right)\left(\omega_{1}-\omega_{2}\right) \cos 2 \varphi\right\}^{1 / 2} .
$$

Clearly, when $\varphi=0$ the spectrum is given by the two lines

$$
E_{k}^{0}(\lambda)=\epsilon_{k}+\lambda \omega_{k} \quad k=1,2
$$

which intersect at the point of degeneracy

$$
\lambda_{0}=-\frac{\left(\epsilon_{1}-\epsilon_{2}\right)}{\left(\omega_{1}-\omega_{2}\right)}
$$

This may be termed the unperturbed problem because there is no coupling between the two levels. The spectrum obtained from (4.15) is called a line spectrum because it consists of two lines crossing one another as depicted in Fig. (4.3a). 
Figure 4.3: (a) Line spectrum of the unperturbed problem with a real degeneracy at $\lambda_{0}$. (b) The degeneracy is lifted when $\varphi$ is switched on and the wave functions interchange association.

When the coupling between the two levels is turned on by switching on $\varphi$ the degeneracy is lifted and an avoided level crossing occurs as shown in Fig. (4.3 b). The two levels now coalesce in the complex $\lambda$-plane where $S$ in (4.14) vanishes and $E_{1}\left(\lambda_{c}\right)=E_{2}\left(\lambda_{c}\right)$, which happens at the complex conjugate points

$$
\lambda_{c}=-\frac{\epsilon_{1}-\epsilon_{2}}{\omega_{1}-\omega_{2}} \exp ( \pm 2 i \varphi) ; \quad|\varphi| \leq \frac{\pi}{2}
$$

At these points, the two levels $E_{1}$ and $E_{2}$ are connected by a square root branch point (exceptional point), in fact the two levels are the values of one analytic function on two different Riemann sheets connected at the square root branch point singularity at $\lambda=\lambda_{c}$. Under variation of $\varphi$, these exceptional points lie on a circle whose radius is determined by a point of degeneracy of the unperturbed problem given in (4.16). The Riemann sheets are only disconnected for values $\varphi=0$ and $\varphi=\pi / 2$ where the two branch point singularities coalesce on the real $\lambda$-axis.

It is instructive to examine the eigenvectors of the problem which are the column vectors of the matrix $B(\lambda)$ that diagonalises $H(\lambda)$. We let $B(0)$ be the unit matrix and $B(\infty)$ equal to $U$ given in (4.2). $B(\lambda)$ can be parameterised by the angle $\varphi(\lambda)$ which is given by

$$
\tan \varphi(\lambda)=\frac{1}{2}\left[\frac{\lambda\left(\omega_{2}-\omega_{1}\right) \sin 2 \varphi}{E_{2}(\lambda)-\epsilon_{1}-\lambda\left(\omega_{1} \cos ^{2} \varphi+\omega_{2} \sin ^{2} \varphi\right)}\right]
$$

so that $\varphi(0)=0$ and $\varphi(\infty)=\varphi$. It is also found that $\varphi(-\infty)=\varphi(\infty)+\pi / 2$. In the vicinity of avoided level crossings $\varphi(\lambda)$ decreases by $\pi / 2$ indicating interchange of the positions of the eigenvectors as described in $\S(4.3)$.

These considerations carry over to an $N$-dimensional problem [HS 90]. The diagonal matrix $D_{0}$ contains the elements $\epsilon_{k}$ and $D_{1}$ the elements $\omega_{k}, k=1, \ldots, N$; the matrix $U$ is now an $N$-dimensional rotation which can be parameterised by $N(N-1) / 2$ angles. The spectrum of $H(\lambda)=D_{0}+\lambda D_{1}$ consists of $N$ unperturbed lines given by $E_{k}^{0}(\lambda)$ with intercepts $\epsilon_{k}$ on the energy axis and slopes of $\omega_{k}$. Generically, these lines 
have $N(N-1) / 2$ intersection points. As before, the degeneracy is lifted by switching on the coupling terms in $H_{1}$ using the $N$-dimensional rotation matrix $U$. This rotation matrix is determined by $N(N-1) / 2$ angles which may be defined in many different ways.

A general rotation in $N$-dimensional space is a product of $N(N-1) / 2$ Jacobi (primitive) rotations of the form

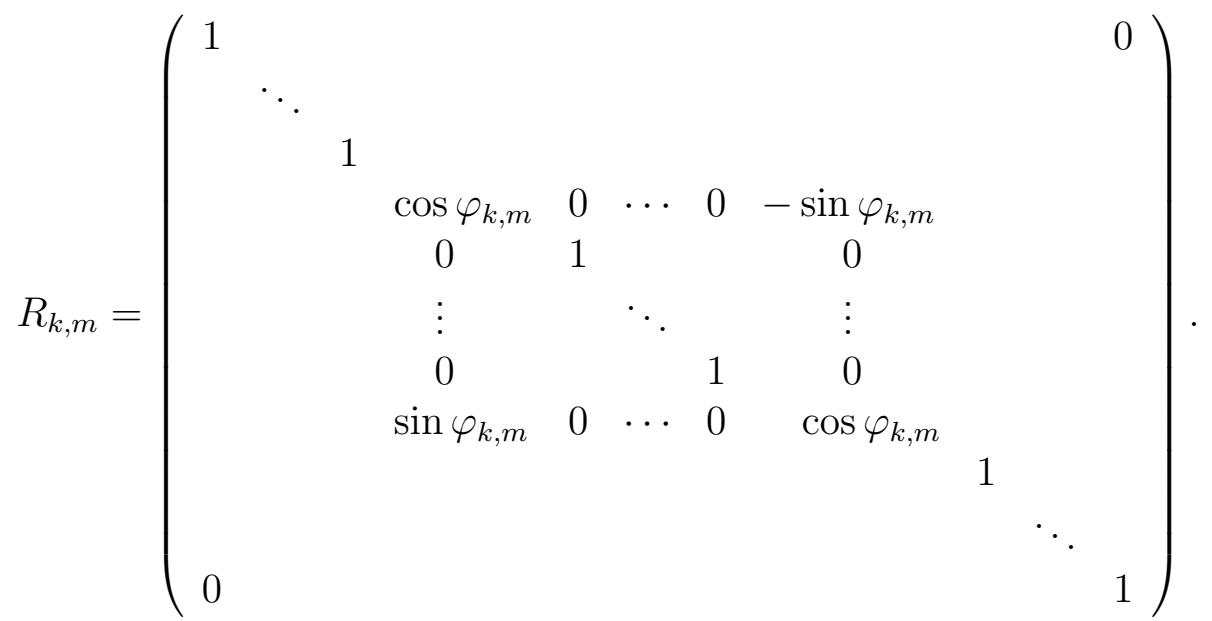

This rotation only affects the $k$-th and $m$-th axes because $\cos \varphi_{k, m}$ and $\sin \varphi_{k, m}$ occur only in the $k$-th and $m$-th row and column. In the special case where $U=R_{k, m}$, a level repulsion occurs between the the two levels $E_{k}^{0}=\epsilon_{k}+\lambda \omega_{k}$ and $E_{m}^{0}=\epsilon_{m}+\lambda \omega_{m}$ that crossed previously, when the angle $\varphi$ is varied from zero. The primitive rotations are therefore chosen to be precisely the ones which have a one-to-one correspondence to all the crossings of the unperturbed lines.

A possible choice is to represent a general rotation by the product of $N(N-1) / 2$ primitive rotations $R_{k, m}$ where $k=1,2, \ldots, N-1$ and $m=k+1, k+2, \ldots, N$. Each of the angles $\varphi_{k, m}$ thus controls the level repulsion of exactly one crossing of the unperturbed lines labelled $k$ and $m$. As long as the angles are infinitesimally small, the order of the product of primitive rotations, which is a second order effect, is immaterial [HS 90].

\subsubsection{Definition of the Angles}

Heiss and Sannino presented an algorithm for defining the angles, of an arbitrary orthogonal matrix, unambiguously [HS 90] (the algorithm is numerically very stable). The angles provide a tool for numerical experimentation whereby the effects of changes in angles size (coupling) on the spectrum can be examined (see $\S 4.6$ ).

Let $U$ be the matrix that diagonalises $H_{1}$ in the basis of diagonal $H_{0}$. The angles $\varphi_{k, m}$ of $U$ is uniquely determined by noting that the order of the Jacobi rotations in (4.19) is important. The order is fixed as follows

$$
U=B_{1} \cdot B_{2} \cdots B_{N-1}
$$


where

$$
B_{m}=R_{m, m+1} \cdot R_{m, m+2} \cdots R_{m, N}
$$

and $R_{j, k}$ is just the Jacobi rotation in (4.19) involving only the $j$-th and $k$-th axes. The set of angles $\varphi_{1,2}, \varphi_{1,3}, \ldots, \varphi_{1, N}$ are determined by identifying the first column of $U$ with the first column vector of matrix $B_{1}$; the angles $\varphi_{2,3}, \varphi_{2,4}, \ldots, \varphi_{2, N}$ can be found in the same way from the second column of the matrix $B_{1}^{-1} U$ (the first column of this matrix has zeros everywhere except for the $1-1$ position which is unity); ...; $\varphi_{N-1, N}$ is determined by $B_{N-2}^{-1} \cdots B_{1}^{-1} U$. For $N=3$ the angles so defined are not equal to the Euler angles [HS 90].

\subsubsection{Effect of the Exceptional Points on the Spectrum}

Globally the Riemann sheet structure can be explained in the following manner: assume that two levels $E_{k}$ and $E_{k+1}$ coalesce at the points $\lambda_{c}$ and $\bar{\lambda}_{c}$. Two functions may be defined

$$
\begin{aligned}
g(\lambda) & =\frac{1}{2}\left(E_{k}(\lambda)+E_{k+1}(\lambda)\right) \\
f(\lambda) & =\frac{E_{k}(\lambda)-E_{k+1}(\lambda)}{2 \sqrt{\left(\lambda-\lambda_{c}\right)\left(\lambda-\bar{\lambda}_{c}\right)}} .
\end{aligned}
$$

Both are regular at $\lambda_{c}$ and $\bar{\lambda}_{c}$, while they have square root branch points at the exceptional points where $E_{k}$ and $E_{k+1}$ are connected to levels with labels different from $k+1$ and $k$, respectively. Rearrangement of the equations in (4.20) shows the connectedness of the two levels. Since both functions $g$ and $f$ are regular at $\lambda_{c}$ and $\bar{\lambda}_{c}$, the singularity structure is displayed, namely

$$
\begin{aligned}
E_{k}(\lambda) & =g(\lambda)+\sqrt{\left(\lambda-\lambda_{c}\right)\left(\lambda-\bar{\lambda}_{c}\right)} f(\lambda) \\
E_{k+1}(\lambda) & =g(\lambda)-\sqrt{\left(\lambda-\lambda_{c}\right)\left(\lambda-\bar{\lambda}_{c}\right)} f(\lambda) .
\end{aligned}
$$

The gap between the energy levels is given by $\Delta=E_{k}-E_{k+1}$. To see the effect of the singularity upon the spectrum for real $\lambda$

$$
\left.\frac{d \Delta}{d \lambda}\right|_{\lambda_{\min }}=0
$$

must be solved. This leads to the equation

$$
2 f(\lambda)\left(\lambda-\Re \lambda_{c}\right)+\left|\lambda-\lambda_{c}\right|^{2} f^{\prime}(\lambda)=0 .
$$

The value of (4.22) in the vicinity of the exceptional point $\lambda_{c}$ can be found. The second term of (4.22) is small under two conditions: Firstly $f^{\prime}$ is expected to be small 
if the nearest singularity of $f$ is not too close and secondly $\left(\lambda-\lambda_{c}\right)$ is small if $\lambda_{c}$ is close to the real axis. If either one of the two conditions (or both) holds we have

$$
\lambda_{\min } \approx \Re \lambda_{c}
$$

which means that an avoided level crossing occurs just at $\Re \lambda_{c}$. The value of the gap is

$$
\Delta \approx 2 f\left(\Re \lambda_{c}\right) \cdot \Im \lambda_{c} .
$$

Note that these relations become exact for $N=2$ as $f$ is constant in this case. The important conclusion to be drawn from the previous discussion is that isolated singularities behave locally like a two dimensional problem.

For $N>2, f^{\prime}\left(\Re \lambda_{c}\right)$ can be large due to a near singularity. This could invalidate the approximate relation given in (4.23). In this context one must be aware of the Riemann sheet structure: if two singularities lie close to one another, or are equal, there mutual influence with regard to the spectrum will depend on whether they lie in the same Riemann sheet. If the exceptional points lie in different sheets, even if they coincide, there is no effect on the avoided level crossing and (4.23) holds. If they closely neighbour one another, or coincide, on the same sheet, $\lambda_{\min } \approx \Re \lambda_{c}$ is invalidated. It is then necessary and generally sufficient to study the three dimensional system which is locally equivalent [HS 91b].

By choosing in the $\lambda$-plane the path of least distance from the real $\lambda$-axis to a particular exceptional point, it is possible to determine which pair of energy levels $\left(E_{i}, E_{k}\right)$ is connected at that branch point. The shape of the spectrum is thus determined. This shows that the exceptional points, and the corresponding sheet structure, determine the statistical properties of the spectrum [ZVW 83].

The level repulsion and corresponding wave functions are very sensitive to any motion of the singularities (under variation of $\lambda$ ) since the derivative $d E / d \lambda$ of the anti-crossing levels in (4.11), is infinity at $\lambda_{c}$ and very large in the neighbourhood of the exceptional points. It is conjectured that this high local sensitivity, of many exceptional points, under perturbation is a signature of quantum chaos [Sa 91]. The global statistical properties, however, remain stable under perturbation.

\subsection{Density of Exceptional Points}

The previous section described how the distribution of the exceptional points determines the fluctuation properties of the level spacings. It was also mentioned in $\S 4.4 .1$ that the calculation of the exceptional points is difficult and time consuming. In this section an efficient method to determine the distribution of exceptional points will be introduced. This is done by using the concept of unperturbed lines described in $\S 4.4 .3$.

The line spectrum preconceives the conjecture that the spectrum of every chaotic system of the form $H_{0}+\lambda H_{1}$ has an underlying simple structure. This structure 
is imposed on the system through the symmetries of $H_{0}$ and $H_{1}$, even when the symmetries are incompatible [HC 92]. When calculating the line spectrum we thus keep the following in mind: the definition of the line spectrum should be kept as simple as possible, preferably, using quantities calculated from $H_{0}$ and $H_{1}$ alone only.

\subsubsection{Line Spectrum: Nondegenerate Systems}

We consider Hamiltonians of the from (see $§ 4.2$ )

$$
H=D_{0}+\lambda U \cdot D_{1} U^{-1}
$$

with $D_{0}$ and $D_{1}$ the diagonal representation of $H_{0}$ and $H_{1}$ respectively and $U \neq 1$. The eigenvalues of $H_{0}$ are given by $\epsilon_{k}$ and that of $H_{1}$ by $\omega_{k}$. We assume that there are no degeneracies in the spectrum of $H$. If $U$ is the unit matrix the spectrum of $H$ is given by the line spectrum of (4.15) with $k=1,2, \ldots, N$. The $N(N-1) / 2$ intersection points of the $N$ lines can be calculated with ease.

The intersection points, however, depend on the relative order of the eigenvalues $\epsilon_{k}$ and $\omega_{k}$. If both sequences are in ascending order, all intersections occur at negative $\lambda$-values; conversely, if one sequence is ascending and the other descending all intersections occur at positive $\lambda$-values. In general the appropriate order for the actual problem is expected to lie between the two extremes.

The appropriate order is given by the asymptotic behaviour of $E_{k}(\lambda)$ of the full problem. The eigenvalues of $H_{0}$ are known for all the systems that we analyse. If $\lambda$ is large in (4.24), $H_{1}$ is seen as the dominant part of the Hamiltonian and $H_{0}$ as a perturbation. The eigenvalues $E_{k}(\lambda)$ of $H$ are therefore approximated with perturbation theory. Because the singularities occur in the complex energy plane at finite values of $\lambda, E_{k}(\lambda)$ is expanded in a Laurent series outside the annulus which contains all the singularities where

$$
E_{k}(\lambda)=\alpha_{k}+\lambda E_{k}^{(1)}+E_{k}^{(2)} / \lambda+O\left(1 / \lambda^{2}\right) ; \quad \lambda \gg 1 .
$$

There are no exceptional points (singularities) near to $\lambda=\infty$ so that the series is convergent. The exceptional point furthest from the origin, however, disturbs the convergence for that specific $k . E_{k}^{(1)}$ are then the eigenvalues of $H_{1}$ i.e. $\omega_{k} . \alpha_{k}$ are the expectation values of $H_{0}$ in the basis of diagonal $H_{1}$ i.e. the diagonal elements of the "backwards" rotated $H_{0}$ viz.

$$
\alpha_{k}=\left(U^{-1} \cdot H_{0} \cdot U\right)_{k, k} .
$$

Because $\lambda$ is large, we neglect higher order terms in (4.25) to give the line spectrum

$$
E_{k}(\lambda)=\alpha_{k}+\lambda \omega_{k}
$$

This is shown as the dashed lines in Fig. (4.4) where $\alpha_{k}$ are the $E$-axis intercepts and $\omega_{k}$ the slopes. The two quantities $\alpha_{k}$ and $\omega_{k}$ are calculated from knowledge of 
Figure 4.4: Asymptotic line spectrum for a system that has systematic degeneracies. Shown here is $E_{j}^{\prime}=\epsilon_{j}+\lambda \delta_{j}$ (solid lines), $E_{k}=\alpha_{k}+\lambda \omega_{k}$ (dashed lines) and the dotted lines represent the joining algebraic equations.

$H_{0}$ and $H_{1}$ alone so that $E_{k}$ in (4.27) are easily calculated; very accurate numerical results can be obtained. The method described above is applied in Chapter 5 to the chaotic quartic oscillator.

Another procedure for obtaining the order of the unperturbed lines was given by Heiss and Sannino [HS 90]. They used the angles $\varphi$ in their proposal, but the procedure proposed here is more efficient to implement and, in fact, the lines as defined here are nearer to the actual spectrum.

\subsubsection{Line Spectrum: Degenerate Systems}

If either $H_{0}$ or $H_{1}$ is degenerate, the number of exceptional points is less than $N(N-$ 1)/2 depending on the degree of degeneracy. If the procedure in the previous section is used however, the degenerate points will appear as intersection points thus giving rise to $N(N-1) / 2$ crossing points. The procedure above thus simulates exceptional points at the points of degeneracy. This problem is overcome by taking the reducibility of the underlying Hilbert space into account.

Let us suppose $H_{0}$ has systematic degeneracies and that the underlying space $\mathcal{E}$ is reducible into $p$ subspaces so that (the hydrogen atom for example)

$$
\mathcal{E}=\mathcal{E}_{1} \oplus \mathcal{E}_{2} \oplus \ldots \oplus \mathcal{E}_{p}
$$

where each subspace has dimension $g_{\mu} ; \mu=1,2, \ldots, p$. Each subspace $\mathcal{E}_{\mu}$ is thus $g_{\mu^{-}}$ fold degenerate with eigenvalues $\epsilon_{\mu}$. We again assume that the eigenvalues of $H_{0}$ are 
known and that $\lambda H_{1}$ is a perturbation. If the perturbation is small, the eigenvalues of $H$ in (4.24) can also be approximated by perturbation theory. In the subspace $\mathcal{E}_{\mu}$, the energy is expanded in a Taylor series around $\lambda=0$ to give

$$
E_{l}^{\prime}=\epsilon_{\mu}+\lambda E_{l}^{(1)}+\lambda^{2} E_{l}^{(2)}+O\left(\lambda^{3}\right) ; \quad \lambda \ll 1
$$

where $l=1,2, \ldots, g_{\mu}$. The second term in the expansion, $E_{l}^{(1)}$, are the eigenvalues of $H_{1}$ and $\epsilon_{\mu}$ those of $H_{0}$ in $\mathcal{E}_{\mu}$. Because $\lambda$ is small we have, up to a first order approximation

$$
E_{l}^{\prime}=\epsilon_{\mu}+\lambda \delta_{l}
$$

with $\delta_{l}$ the eigenvalues of $H_{1}$ in $\mathcal{E}_{\mu}$, ordered ascendingly. This is shown as one bunch of levels originating at $\epsilon_{\mu}$ with slopes $\delta_{l}$ in Fig. (4.4). There is thus a line spectrum in the form of (4.28) for each subspace.

The asymptotic line spectrum for the system as a whole is obtained by taking all the different line spectra for each subspace together so that

$$
E_{j}^{\prime}=\epsilon_{j}+\lambda \delta_{j} ; \quad j=1,2, \ldots, N
$$

This is shown as the solid lines on the left side of Fig. (4.4). Note that $\epsilon_{j}$ are ordered in ascending order. The order of $\delta_{j}$ is obtained by noting the following: let $\delta_{1}^{2}$ be the lowest eigenvalue of $H_{1}$ in the subspace $\mathcal{E}_{2}$. The order of the $\delta$ 's is then

$$
\delta_{j}=\delta_{1}^{1}, \delta_{2}^{1}, \ldots, \delta_{g_{1}}^{1}, \delta_{1}^{2}, \ldots, \delta_{g_{2}}^{2}, \ldots, \delta_{g_{p}}^{p} .
$$

$N$ is the dimension of the system where $N=g_{1}+g_{2}+\ldots+g_{p}$. Note that the line spectrum is again obtainable from quantities easily available from $H_{0}$ and $H_{1}$ alone.

If $H_{1}$ is nondegenerate (4.27) still holds when $\lambda \gg 1$ and (4.29) and (4.27) are approximations of the actual spectrum at the asymptotic values of $\lambda$. The total line spectrum is obtained through joining (4.29) and (4.27) with an algebraic equation that only depends on the four quantities $\epsilon_{k}, \delta_{k}, \alpha_{k}$ and $\omega_{k}$. The form of this equation is determined by the specific form of the spectrum and is represented by the dotted lines in Fig. (4.4). Care has to be taken to connect the appropriate $E_{k}$ in (4.27) with the appropriate $E_{k}^{\prime}$ in (4.29). A general procedure is not at hand and the association is determined by the system and the form of the spectrum. The method above is applied in Chapter 6 to the hydrogen atom in a strong uniform magnetic field.

\subsubsection{Distribution of Exceptional Points}

The significance of (4.27) and (4.29) lies in the easy availability of the parameters of the unperturbed lines from the knowledge of $H_{0}$ and $H_{1}$ alone. The line spectrum approximates the real spectrum so that the exceptional points of the full problem are expected to lie near to the intersection points of the unperturbed lines (for the two 
dimensional problem the real parts of the exceptional points coincide with the point of intersection).

Because the unperturbed line spectrum consists of a set of lines having actual crossings, the intersection points can easily be calculated. If the system is nondegenerate the line spectrum is given by (4.27) and the $\lambda$-values of the $N(N-1) / 2$ intersection points are

$$
\lambda_{k}=\frac{\alpha_{j}-\alpha_{i}}{\omega_{i}-\omega_{j}} ; j>i=1,2, \ldots, N ; \quad k=1,2, \ldots, N(N-1) / 2 .
$$

By choosing a certain bin length in $\lambda$, the density of the intersection points (number distribution) can be calculated and this is taken to be statistically equivalent to the density of the actual exceptional points. Now, because the exceptional points determine the form of the spectrum, and hence the fluctuations, the number distribution is a measure of the chaotic behaviour of the system.

Guhr and Weidenmüller argued that there are two physical quantities that play an important role in the occurrence of quantum chaos: the density of states, $d$, and coupling matrix elements, $C$. The fluctuations are dependent on the product, $C \cdot d$ [GW 90]. From the arguments above the size of the coupling matrix elements are essentially determined by the angles $\varphi$ defined in $\S 4.4 .3$. The density of states is inversely equal to the average level spacing in the system i.e.

$$
d=\frac{1}{\text { mean level spacing }} .
$$

In working with the line spectrum of a nondegenerate system, the density of crossings monotonically increases with the density of states. In fact, the exceptional points of a chaotic system are essentially distributed in the range of $\lambda$-values where chaos is discerned. Likewise, the crossings of the lines are seemingly randomly distributed in that region which is attained if the lines are themselves randomly distributed. The line spectrum then has a Poissonic nearest neighbour spacings distribution (after unfolding). This is further evidence that the density of exceptional points (or density of crossings) is a measure of the chaotic behaviour of the system.

From this discussion it is conjectured that the higher the density of exceptional points, the more likely chaotic behaviour is to occur. The coupling matrix elements play an important role which is evident from the aforementioned arguments: The nearest neighbour spacing distribution of the lines, without coupling, is Poissonic but, by turning on the interaction $(\varphi>0)$ we obtain a spectrum obeying GOE statistics. It is noted that situations can occur where the density of intersection points is high, but the coupling is minute so that chaotic behaviour is suppressed (see Chapter 6). Hence, knowledge of $H_{0}$ and $H_{1}$ alone enables us to predict for which values of $\lambda$ strong fluctuations of the energy levels are expected to occur and where they certainly do not occur. This will be shown with the help of two simple examples in the following section and also in model Hamiltonians introduced in subsequent chapters. 


\subsection{Simple Examples}

In order to elucidate the concepts introduced in the previous sections, we discuss two very simple matrix models which can be considered as the extreme ends of a variety of intermediate possibilities. Through the simplicity of these mathematical models the concepts of unperturbed lines and exceptional points will be exemplified to obtain a better comprehension of the various structures involved in the study of quantum chaos.

These examples generate typical GOE-type level statistics as well as the associated expected statistical properties of the state vectors [HS 91a]. The one example produces a maximum density of exceptional points within a small window of real $\lambda$-values while the other example produces a diluted density over a wide range of real $\lambda$-values.

\subsubsection{High density of exceptional points}

Consider a Hamiltonian of the form [HK 91], [HS 91a]

$$
H=H_{0}+\lambda H_{1} \text {. }
$$

Here, $H_{0}$ and $H_{1}$ are chosen to be diagonal matrices where ( $N$ even)

$$
H_{0}=k \delta_{k, k^{\prime}} \quad \text { and } \quad H_{1}=D_{1}=\left(\frac{N}{2}-k\right) \delta_{k, k^{\prime}} \quad k=1, \ldots, N .
$$

The spectrum is given by the lines

$$
E_{k}(\lambda)=k+\lambda\left(\frac{N}{2}-k\right)
$$

which all intersect at $\lambda=1$ as shown in Fig. (4.5). The fact that we have chosen a harmonic spectrum for both Hamiltonians is immaterial for the following. We now rotate $D_{1}$ by an orthogonal matrix $U$ constructed as described in $\S(4.4 .3)$. It is expected that $N(N-1) / 2$ complex conjugate pairs of exceptional points will emerge from the common intersection point. Clearly the result will depend on the specific choice of the rotation. By choosing the angles random from an interval $\left(-\varphi_{0}, \varphi_{0}\right), U$ can be seen as a random orthogonal matrix so that the strength of the coupling is determined by the length of the interval $\left(-\varphi_{0}, \varphi_{0}\right)$.

It turns out that for $N=400$ the nearest neighbour spacing distribution of the energy levels matches the Wigner surmise for angles as small as $\varphi_{0}=0.003$ where $U$ is still very close to a unit matrix. Likewise, the $\bar{\Delta}_{3}(L)$ curve, which was investigated for $1 \leq L \leq 12$ moves up from the constant value $\bar{\Delta}_{3}=1 / 12$ to its GOE prediction with increasing $\varphi_{0}$ in a corresponding way. We stress the rather sudden change from a harmonic spectrum to a GOE type situation. For $\varphi_{0}=0.003$ these results hold 
Figure 4.5: Unperturbed spectrum of the example discussed in $\S 4.6 .1$ with all lines intersecting at $\lambda=1$ (from [HS 91a]).

only in the immediate vicinity of $\lambda=1$ while the harmonic nature of the spectrum is left virtually unchanged for $\lambda$-values further away from unity. The level statistics are based on unfolded spectra.

The behaviour of the eigenvectors is in accordance with the observations for the spectrum. The eigenvectors are almost identical to the unperturbed ones for $\lambda \neq 1$. In other words, when ordering the eigenvectors according to an ascending order of the eigenvalues, they form for $\lambda<1$ essentially a unit matrix while for $\lambda>1$ their order is simply reversed (up to possible minus signs). Note the for $\lambda \rightarrow \infty$ the exact form is

$$
P(\infty)=U\left[\begin{array}{lll}
0 & & \pm 1 \\
& . \cdot & \\
\pm 1 & & 0
\end{array}\right]
$$

where $U \approx 1$.

For $\lambda \approx 1$ the eigenvectors change dramatically. The strong mixing of the states, brought about by the immediate vicinity of a high density of exceptional points, effects a drastic delocalisation as shown in Fig. (4.6). In the vicinity of $\lambda \approx 1$, the average delocalisation length (see $\S 3.7), l_{d}$, is nearly unity while otherwise it is virtually zero corresponding to the localised eigenvectors which are essentially of the form $(0 \ldots 1 \ldots 0)$ [HK 91]. The width of the bell shaped curve increases with the maximum angle $\varphi_{0}$. It is of interest to look at the fluctuations of the individual lengths. Their distribution is plotted for $\lambda=1$ in Fig. (4.7) showing that a few eigenvectors are localised while others are completely spread out [HK 91].

These results are significant for a number of reasons. We note the extremely high sensitivity under variation of the maximal angle $\varphi_{0}$. If another set of random numbers is used for the angles, the local values of the spectrum and the eigenvectors are strongly affected, yet the statistical properties are left unchanged. The same holds 
Figure 4.6: Average delocalisation length for $\varphi_{0}=0.003$ and a high density of exceptional points.

Figure 4.7: Distribution of individual localisation lengths for $\lambda=1$. 
under variation of $\varphi_{0} \geq 0.003$ at $\lambda=1$.

Because $\varphi_{0}$ is so small, $U$ is still close to a unit matrix so that $H_{0}=U \cdot D_{0} \cdot U^{-1}$ is still close to a diagonal matrix. All the intersection points of the unperturbed lines then occur in the close vicinity of the original crossing point at $\lambda=1$. This corresponds to a high density of exceptional points near $\lambda=1$. This model shows that even though the coupling is small, the parameter $C \cdot d$ is large to ensure chaotic behaviour in the vicinity of $\lambda=1$.

If $\varphi_{0}$ is increased further and further, $U$ will no longer be close to a unit matrix and $H_{1}$ will become a full matrix. The intersection points are then diluted throughout the whole of the admitted $\lambda$ range. This ensures that the unperturbed lines form a seemingly random set in that the intercepts are randomly distributed and so also the actual exceptional points. This is the starting point for the second model where we have a set of lines randomly distributed. Here again $U \approx 1$ so that $\varphi$ is small.

\subsubsection{Diluted density of exceptional points}

We start with the Hamilton matrices

$$
H_{0}=k \delta_{k, k^{\prime}} \quad \text { and } \quad H_{1}=D=\mathcal{P}\left(\frac{N}{2}-k\right) \delta_{k, k^{\prime}} \quad k=1, \ldots, N
$$

where $\mathcal{P}(n)$ is a random permutation of the natural numbers $n$. The spectrum is given by the lines

$$
E_{k}^{0}=k+\lambda \mathcal{P}\left(\frac{N}{2}-k\right)
$$

and is drawn in Fig. (4.8). The corresponding nearest neighbour spacing distribution is a Poisson distribution for a large range of $\lambda$-values $(\lambda=0$ excluded). The eigenvectors are all of the form $(0 \ldots 1 \ldots 0)$ but their sequence (the column vectors) in the diagonalising matrix is randomly distributed. Only for $\lambda>\lambda_{\max }$ are they ordered as in the previous example; by $\lambda_{\max }$ we denote the right most intersection point of the levels.

When rotating $H_{1}$ to produce level repulsion it now turns out that a considerably larger value $\varphi_{0}$ is needed to change the Poisson distribution into a Wigner curve. Only for $\varphi_{0}=0.05$ is the transition about to take place $(N=400)$. The $\bar{\Delta}_{3}(L)$ curve, when plotted for $1 \leq L \leq 15$, has moved down nicely with increasing $\varphi_{0}$ from the straight line $L / 15$ to its GOE prediction. In this example the change from the ordered to the chaotic situation is much less dramatic than in the previous example.

The value $\varphi_{0}=0.05$ implies that $U$ is no longer close to a unit matrix. As a consequence, when comparing with the lines for $\varphi_{0}=0$ the unperturbed lines are reshuffled since the order of the $\alpha_{k}$ is different from the order for $\epsilon_{k}=k$. However, while this substantially affects the individual positions of the intersection points, it does not change their distribution and thus the distribution of the exceptional points. As a consequence, the level statistics hold now for a large range of $\lambda$-values. The 
Figure 4.8: Unperturbed spectrum of the example discussed in $\S 4.6 .2$. The lines are randomly distributed (from [HS 91a]).

consequence for the eigenvector statistics is of a similar nature in that delocalisation is found for all $\lambda>0$. From Figs. (4.9) and (4.10) we see that for $\varphi_{0}=0.05$ the delocalisation is not as strongly pronounced as in the previous example. The reason for this is because the exceptional points are spread over the whole of the $\lambda$ range so that the density all over is much less than for the previous example. Note that the distribution in Fig. (4.10) shows virtually no contribution for $l_{H}>0.6$. The delocalisation does become stronger when $\varphi_{0}$ is further increased.

It is interesting to note the pattern in Fig. (4.9): as indicated by the maximum the mixing for finite $\lambda$-values brings about a more pronounced delocalisation than the one enforced by $U$ for large $\lambda$-values. Here we refer to an important difference to the previous example in that delocalisation persists even for $\lambda \rightarrow \infty$. The eigenvectors appear in a matrix like the one in (4.34) but now with a $U$ that effects delocalisation. The levels, however, become equidistant for $\lambda \gg \lambda_{\max }$ (up to $(1 / \lambda)$ ).

In this model the density of exceptional points is considerably less (and more spread out) than that of the previous example. In order to achieve chaotic behaviour, the coupling has to be much larger to obtain a sufficient large value for $C \cdot d$.

\subsection{Summary}

This chapter introduced and illuminated the concepts of avoided level crossings and exceptional points. We described how the exceptional points determine the form of the spectrum and the subsequent fluctuation patterns. To understand the chaotic behaviour of a spectrum it is thus essential to understand the substructure that brings about the specific form of the spectrum e.g. the exceptional points. An efficient method to determine the density of the exceptional points was introduced using the concept of 'unperturbed lines'. It was shown that the distribution is primarily 
Figure 4.9: Average delocalisation length for $\varphi_{0}=0.05$ and the diluted density of exceptional points.

Figure 4.10: Individual delocalisation length distribution of the diluted density of exceptional points at $\lambda=2$. The qualitative pattern persists for a large range of $\lambda$ values. 
governed by the intersection points of these lines and secondarily by the values of the angles associated with $U$ (the coupling). Because of the simplicity of the outlined procedures, their numerical implementations are straight forward and highly accurate results were obtained.

It is conjectured that the higher the density of exceptional points, the more likely chaotic behaviour is to occur. It was also shown that the exceptional points have a high local sensitivity under perturbation which was conjectured to be a signature of quantum chaos. These conjectures were substantiated with the help of two simple model Hamiltonians.

From the results given in this chapter it seems that, if the Hamiltonian is of the form $H_{0}+\lambda H_{1}$, a possible characterisation of quantum chaos hinges on the distribution of the exceptional points. The concepts introduced in this chapter will now be used to investigate model Hamiltonian systems to be introduced in the next chapters. These concepts will help to get a better comprehension of the behaviour of the systems which have classical chaotic counterparts.

æ 


\section{Chapter 5}

\section{The Quartic Oscillator}

\subsection{Introduction}

The fascinating properties of systems that are the quantal analogue of classical chaotic systems still awaits thorough comprehension. In order to understand the common properties of the quantal operators that give rise to such intricate behaviour, it is instructive to investigate model systems.

In this Chapter we turn to the quartic oscillator. This system has been thoroughly investigated by many authors (see text for references) from various viewpoints. We will ask the question: if a matrix representation of a Hamiltonian, that originates from a classically chaotic analogy is given, what is the mathematical mechanism that yields the special features of the spectrum? This question will be answered through an investigation of the exceptional points of the matrix problem and their effect on the spectrum and eigenfunctions.

In the first part we introduce the system and then list the results from the classical analysis. The system is then quantised and the reduced matrix representation given. A thorough statistical investigation of the eigenvalues and eigenfunctions will lead to the known conclusion that this system shows features ascribed to quantum chaos. In the second last part the exceptional points are studied, and the relation to quantum chaos stated. The last part elaborates on a possible connection between the exceptional points and the semiclassical results.

\subsection{The Model}

The Hamiltonian we study is given by [SLK 88]

$$
H_{\alpha}(\mathbf{p}, \mathbf{q})=\frac{1}{2}\left(p_{1}^{2}+p_{2}^{2}\right)+V_{\alpha}(\mathbf{q})
$$


with

$$
V_{\alpha}(\mathbf{q})=\frac{1-\alpha}{12}\left(q_{1}^{4}+q_{2}^{4}\right)+\frac{1}{2} q_{1}^{2} q_{2}^{2} ; \quad \alpha \leq 1
$$

There are two reasons for studying this system: firstly, by varying the parameter $\alpha$ the system can be changed continuously from an integrable case towards one regarded as "completely chaotic" in the sense that almost all trajectories explore the entire energy surface [CP 84]. Secondly, the fact that the potential $V(\mathbf{q})$ is homogeneous implies scaling properties for $H$ and the classical analysis is needed only on a single energy surface. The contours for the potential are illustrated in Fig. (5.1) for various values of $\alpha$ and energy $E=1$.

When $\alpha=1$

$$
H=\frac{1}{2}\left(p_{1}^{2}+p_{2}^{2}+q_{1}^{2} q_{2}^{2}\right)
$$

This Hamiltonian has been suggested as a simplified zero dimensional model of the classical Yang-Mills field and has been studied by Steeb et al [SVK 85] and Martens et al [MWR 89]. Savvidy showed that this system is a Kolmogorov system (K-system) and it can be viewed as the simplest Hamiltonian showing real chaotic behaviour ${ }^{1}$ with equations of motion [Sa 84], [Ar 80]

$$
\ddot{x}_{1}=-x_{1} x_{2}^{2}, \quad \ddot{x}_{2}=-x_{1}^{2} x_{2} .
$$

An interesting aspect is the fact that even though the classical phase space of the system in (5.3) is noncompact and the energy surface infinite, quantally the spectrum is discrete [Si 83], [Ec 88].

\subsection{Classical Analysis}

The classical system has been thoroughly investigated by Carnegie and Percival [CP 84] while Pullen and Edmonds studied a related system [PE 81]. $H$ in (5.1) is integrable when $\alpha=0$ but Carnegie and Percival found that the motion is always irregular for $\alpha>0$. This system shows a transition from a completely integrable system to a K-system with increasing $\alpha$. The proof is found through the Lyaponov exponent [Ko 88b] drawn in Fig. (5.2) and the Poincaré surface of section drawn in Fig. (5.3) for a few values of $\alpha$. This shows that at $\alpha=0.8$ there are still two obvious regular regions (outlined in Fig. $(5.3 \mathrm{c}$ )) but beyond $\alpha=0.93$ there seem to be no regular regions left and the system is fully chaotic.

Dahlqvist and Russberg, however, found that even when $\alpha=1$, there are some stable periodic orbits giving rise to tiny regular islands [DR 90]. The investigation is only necessary for $\alpha \in[-1,1]$ because $\alpha \in[-\infty,-1)$ can be mapped into the range $[-1,1]$ with $\alpha=-\infty$ corresponding to $\alpha=1$ (in general $\alpha \leftrightarrow(3+\alpha) /(\alpha-1)$ ) [Bo 92a]. When $\alpha=-1$ the system has rotational symmetry where $V \sim q^{4}$.

\footnotetext{
${ }^{1}$ Quartic oscillators avoid the nongeneric properties of harmonic oscillators.
} 
Figure 5.1: The potential in (5.2) for $E=1$ and (a) $\alpha=0$, (b) $\alpha=0.25$, (c) $\alpha=0.8$ and (d) $\alpha=1$ (from [CP 84]).

Figure 5.2: The Lyaponov exponent for the quartic oscillator. From this it is evident that chaos sets in at $\alpha \approx 0.15$. 
Figure 5.3: Poincaré surface of section for the quartic oscillator for (a) $\alpha=0$, (b) $\alpha=0.25$, (c) $\alpha=0.8$ (the two obvious regular regions are outlined) and (d) $\alpha=0.93$ (from [CP 84]).

\subsection{Matrix Representation of Quantum System}

For our purposes $H_{\alpha}$ is rotated to new variables $(\tilde{q}, \tilde{p})$ and then scaled to be in the following form (see Appendix A)

$$
H\left(\tilde{p}_{1}, \tilde{p}_{2}, \tilde{q}_{1}, \tilde{q}_{2}\right)=H_{0}\left(\tilde{p}_{1}, \tilde{p}_{2}, \tilde{q}_{1}, \tilde{q}_{2}\right)+\lambda H_{1}\left(\tilde{q}_{1}, \tilde{q}_{2}\right)
$$

where

$$
\begin{aligned}
& H_{0}=\frac{1}{2}\left[\tilde{p}_{1}^{2}+\tilde{p}_{2}^{2}\right]+\frac{1}{6}\left[\tilde{q}_{1}^{4}+\tilde{q}_{2}^{4}\right] \\
& H_{1}=-\frac{1}{3} \tilde{q}_{1}^{2} \tilde{q}_{2}^{2} .
\end{aligned}
$$

The matrix representation we use is obtained by introducing Bose creation and annihilation operators defined as follows

$$
b_{k}^{\dagger}=\frac{1}{\sqrt{2}}\left(\tilde{q}_{k}-i \tilde{p}_{k}\right) ; \quad b_{k}=\frac{1}{\sqrt{2}}\left(\tilde{q}_{k}+i \tilde{p}_{k}\right) .
$$

With the two dimensional Harmonic Oscillator basis

$$
\left|m_{1}, m_{2}\right\rangle:=\frac{1}{\sqrt{m_{1} ! m_{2} !}}\left(b_{1}^{\dagger}\right)^{m_{1}}\left(b_{2}^{\dagger}\right)^{m_{2}}|0\rangle
$$


in the infinite dimensional Hilbert space $\ell_{2}(N)$ (in number representation), we obtain the matrix representations of $H_{0}$ and $H_{1}$ viz.

$$
\begin{aligned}
H_{k l}^{0} & =\left[\frac{3}{4}+\frac{1}{4}\left(m_{1}^{2}+m_{2}^{2}\right)+\frac{3}{4}\left(m_{1}+m_{2}\right)\right] \delta_{r_{1}, m_{1}} \delta_{r_{2}, m_{2}} \\
& +\frac{m_{1}}{6} \sqrt{\left(m_{1}+1\right)\left(m_{1}+2\right)} \delta_{r_{1}, m_{1}+2} \delta_{r_{2}, m_{2}}+\frac{m_{2}}{6} \sqrt{\left(m_{2}+1\right)\left(m_{2}+2\right)} \delta_{r_{1}, m_{1}} \delta_{r_{2}, m_{2}+2} \\
& +\frac{m_{1}-2}{6} \sqrt{m_{1}\left(m_{1}-1\right)} \delta_{r_{1}, m_{1}-2} \delta_{r_{2}, m_{2}}+\frac{m_{2}-2}{6} \sqrt{m_{2}\left(m_{2}-1\right)} \delta_{r_{1}, m_{1}} \delta_{r_{2}, m_{2}-2} \\
& +\frac{1}{24} \sqrt{\left(m_{1}+1\right)\left(m_{1}+2\right)\left(m_{1}+3\right)\left(m_{1}+4\right)} \delta_{r_{1}, m_{1}+4} \delta_{r_{2}, m_{2}} \\
& +\frac{1}{24} \sqrt{\left(m_{2}+1\right)\left(m_{2}+2\right)\left(m_{2}+3\right)\left(m_{2}+4\right)} \delta_{r_{1}, m_{1}} \delta_{r_{2}, m_{2}+4} \\
& +\frac{1}{24} \sqrt{m_{1}\left(m_{1}-1\right)\left(m_{1}-2\right)\left(m_{1}-3\right)} \delta_{r_{1}, m_{1}-4} \delta_{r_{2}, m_{2}} \\
& +\frac{1}{24} \sqrt{m_{2}\left(m_{2}-1\right)\left(m_{2}-2\right)\left(m_{2}-3\right)} \delta_{r_{1}, m_{1}} \delta_{r_{2}, m_{2}-4}
\end{aligned}
$$

and

$$
\begin{aligned}
H_{k l}^{1} & =\frac{-1}{12}\left\{\left[\left(2 m_{1}+1\right)\left(2 m_{2}+1\right)\right] \delta_{r_{1}, m_{1}} \delta_{r_{2}, m_{2}}\right. \\
& +\left(2 m_{2}+1\right) \sqrt{\left(m_{1}+1\right)\left(m_{1}+2\right)} \delta_{r_{1}, m_{1}+2} \delta_{r_{2}, m_{2}} \\
& +\left(2 m_{1}+1\right) \sqrt{\left(m_{2}+1\right)\left(m_{2}+2\right)} \delta_{r_{1}, m_{1}} \delta_{r_{2}, m_{2}+2} \\
& +\left(2 m_{2}+1\right) \sqrt{m_{1}\left(m_{1}-1\right)} \delta_{r_{1}, m_{1}-2} \delta_{r_{2}, m_{2}} \\
& +\left(2 m_{1}+1\right) \sqrt{m_{2}\left(m_{2}-1\right)} \delta_{r_{1}, m_{1}} \delta_{r_{2}, m_{2}-2} \\
& +\sqrt{\left(m_{1}+1\right)\left(m_{1}+2\right)\left(m_{2}+1\right)\left(m_{2}+2\right)} \delta_{r_{1}, m_{1}+2} \delta_{r_{2}, m_{2}+2} \\
& +\sqrt{\left(m_{1}+1\right)\left(m_{1}+2\right)\left(m_{2}-1\right)\left(m_{2}-2\right)} \delta_{r_{1}, m_{1}+2} \delta_{r_{2}, m_{2}-2} \\
& +\sqrt{m_{1}\left(m_{1}-1\right)\left(m_{2}+1\right)\left(m_{2}+2\right)} \delta_{r_{1}, m_{1}-2} \delta_{r_{2}, m_{2}+2} \\
& \left.+\sqrt{m_{1} m_{2}\left(m_{1}-1\right)\left(m_{2}-1\right)} \delta_{r_{1}, m_{1}-2} \delta_{r_{2}, m_{2}-2}\right\} .
\end{aligned}
$$

Here $m_{1}, m_{2}=0,1,2, \ldots, N-1$ where we choose the order of the number pairs $\left(m_{1}, m_{2}\right)$ to be

$$
(0,0),(1,0),(0,1),(2,0),(1,1),(0,2),(3,0),(2,1),(1,2),(0,3), \ldots,(0, N-1) .
$$

$N$ is the number of energy shells considered. Remember that we work in an infinite dimensional space and are thus forced to truncate the matrices in (5.5) and (5.6). The dimension of the truncated matrices, with the aforementioned ordering of the number pairs, is

$$
M=\frac{N(N+1)}{2}
$$


and the subscripts of the matrices are $k, l=1,2, \ldots, M$.

The spectrum of $H$ in (5.4) contains degeneracies due to the symmetric character of $H$. The symmetries are the same as those for the $C_{4 v}$ point group. All the symmetries have to be reduced out before the spectrum can be studied. This is done with Group Theoretical techniques using the symmetries of the $C_{4 v}$ point group as outlined in Appendix A. We consider the positive parity states and of those the states symmetric under permutation of $\tilde{p}_{1} \rightarrow \tilde{p}_{2}$ and $\tilde{q}_{1} \rightarrow \tilde{q}_{2}$. This is given by the $A_{1}$ subspace with the basis ${ }^{2}$ (see (A.23))

$$
\left|m_{1}, m_{2}\right\rangle=\left[2+2 \delta_{m_{1}, m_{2}}\right]^{1 / 2}\left(\left|2 m_{1}, 2 m_{2}\right\rangle+\left|2 m_{2}, 2 m_{1}\right\rangle\right)
$$

We note that, by using this basis, the number pairs $\left(m_{1}, m_{2}\right)$ and $\left(m_{2}, m_{1}\right)$ will lead to the same state. To avoid this, the order of the number pairs $\left(m_{1}, m_{2}\right)$ is taken to be

$$
(0,0),(1,0),(2,0),(1,1),(3,0), \ldots, \begin{cases}(N-1,0), \ldots,\left(\frac{N-1}{2}, \frac{N-1}{2}\right) & N \text { odd } \\ (N-1,0), \ldots,\left(\frac{N}{2}, \frac{N}{2}-1\right) & N \text { even }\end{cases}
$$

where the dimension of the truncated matrices is now given by

$$
M= \begin{cases}\frac{N}{2}\left(\frac{N}{2}+1\right) & N \text { odd } \\ \left(\frac{N+1}{2}\right)^{2} & N \text { even. }\end{cases}
$$

The number pairs in (5.7) and the dimension $M$ in (5.8) will always ensure that the last energy shell is contained completely.

With the order in (5.7), the matrix representations of $H_{0}$ and $H_{1}$ in the $A_{1}$ subspace is given viz.

$$
\begin{aligned}
H_{k l}^{0} & =\frac{1}{24}\left[\left(1+\delta_{m_{1}, m_{2}}\right)\left(1+\delta_{r_{1}, r_{2}}\right)\right]^{1 / 2} \times \\
& \times\left\{\left[24\left(m_{1}^{2}+m_{2}^{2}\right)+36\left(m_{1}+m_{2}\right)+18\right]\left[\delta_{r_{1}, m_{1}} \delta_{r_{2}, m_{2}}+\delta_{r_{1}, m_{2}} \delta_{r_{2}, m_{1}}\right]\right. \\
& +8 m_{1} \sqrt{\left(2 m_{1}+1\right)\left(2 m_{1}+2\right)}\left[\delta_{r_{1}, m_{1}+1} \delta_{r_{2}, m_{2}}+\delta_{r_{1}, m_{2}} \delta_{r_{2}, m_{1}+1}\right] \\
& +8 m_{2} \sqrt{\left(2 m_{2}+1\right)\left(2 m_{2}+2\right)}\left[\delta_{r_{1}, m_{1}} \delta_{r_{2}, m_{2}+1}+\delta_{r_{1}, m_{2}+1} \delta_{r_{2}, m_{1}}\right] \\
& +8\left(m_{1}-1\right) \sqrt{2 m_{1}\left(2 m_{1}-1\right)}\left[\delta_{r_{1}, m_{1}-1} \delta_{r_{2}, m_{2}}+\delta_{r_{1}, m_{2}} \delta_{r_{2}, m_{1}-1}\right]+
\end{aligned}
$$

\footnotetext{
${ }^{2}$ Bohigas used semiclassical logic to introduce another basis set that gives better accuracy and ensures exponential convergence of the energy levels [Bo 92a].
} 


$$
\begin{aligned}
& +8\left(m_{2}-1\right) \sqrt{2 m_{2}\left(2 m_{2}-1\right)}\left[\delta_{r_{1}, m_{1}} \delta_{r_{2}, m_{2}-1}+\delta_{r_{1}, m_{2}-1} \delta_{r_{2}, m_{1}}\right] \\
& +\sqrt{\left(2 m_{1}+1\right)\left(2 m_{1}+2\right)\left(2 m_{1}+3\right)\left(2 m_{1}+4\right)}\left[\delta_{r_{1}, m_{1}+2} \delta_{r_{2}, m_{2}}+\delta_{r_{1}, m_{2}} \delta_{r_{2}, m_{1}+2}\right] \\
& +\sqrt{\left(2 m_{2}+1\right)\left(2 m_{2}+2\right)\left(2 m_{2}+3\right)\left(2 m_{2}+4\right)}\left[\delta_{r_{1}, m_{1}} \delta_{r_{2}, m_{2}+2}+\delta_{r_{1}, m_{2}+2} \delta_{r_{2}, m_{1}}\right] \\
& +\sqrt{2 m_{1}\left(2 m_{1}-1\right)\left(2 m_{1}-2\right)\left(2 m_{1}-3\right)}\left[\delta_{r_{1}, m_{1}-2} \delta_{r_{2}, m_{2}}+\delta_{r_{1}, m_{2}} \delta_{r_{2}, m_{1}-2}\right] \\
& \left.+\sqrt{2 m_{2}\left(2 m_{2}-1\right)\left(2 m_{2}-2\right)\left(2 m_{2}-3\right)}\left[\delta_{r_{1}, m_{1}} \delta_{r_{2}, m_{2}-2}+\delta_{r_{1}, m_{2}-2} \delta_{r_{2}, m_{1}}\right]\right\}
\end{aligned}
$$

and

$$
\begin{aligned}
H_{k l}^{1} & =\frac{-1}{12}\left[\left(1+\delta_{m_{1}, m}\right)\left(1+\delta_{r_{1}, r_{2}}\right)\right]^{1 / 2} \times \\
& \times\left\{\left[4\left(m_{1}+m_{2}\right)+16 m_{1} m_{2}+1\right]\left[\delta_{r_{1}, m_{1}} \delta_{r_{2}, m_{2}}+\delta_{r_{1}, m_{2}} \delta_{r_{2}, m_{1}}\right]\right. \\
& +\left(1+4 m_{2}\right) \sqrt{\left(2 m_{1}+1\right)\left(2 m_{1}+2\right)}\left[\delta_{r_{1}, m_{1}+1} \delta_{r_{2}, m_{2}}+\delta_{r_{1}, m_{2}} \delta_{r_{2}, m_{1}+1}\right] \\
& +\left(1+4 m_{1}\right) \sqrt{\left(2 m_{2}+1\right)\left(2 m_{2}+2\right)}\left[\delta_{r_{1}, m_{1}} \delta_{r_{2}, m_{2}+1}+\delta_{r_{1}, m_{2}+1} \delta_{r_{2}, m_{1}}\right] \\
& +\left(1+4 m_{2}\right) \sqrt{2 m_{1}\left(2 m_{1}-1\right)}\left[\delta_{r_{1}, m_{1}-1} \delta_{r_{2}, m_{2}}+\delta_{r_{1}, m_{2}} \delta_{r_{2}, m_{1}-1}\right] \\
& +\left(1+4 m_{1}\right) \sqrt{2 m_{2}\left(2 m_{2}-1\right)}\left[\delta_{r_{1}, m_{1}} \delta_{r_{2}, m_{2}-1}+\delta_{r_{1}, m_{2}-1} \delta_{r_{2}, m_{1}}\right] \\
& +\sqrt{\left(2 m_{1}+1\right)\left(2 m_{1}+2\right)\left(2 m_{2}+1\right)\left(2 m_{2}+2\right)}\left[\delta_{r_{1}, m_{1}+1} \delta_{r_{2}, m_{2}+1}+\delta_{r_{1}, m_{2}+1} \delta_{r_{2}, m_{1}+1}\right] \\
& +\sqrt{\left(2 m_{1}+1\right)\left(2 m_{1}+2\right) 2 m_{2}\left(2 m_{2}-1\right)}\left[\delta_{r_{1}, m_{1}+1} \delta_{r_{2}, m_{2}-1}+\delta_{r_{1}, m_{2}-1} \delta_{r_{2}, m_{1}+1}\right] \\
& +\sqrt{2 m_{1}\left(2 m_{1}-1\right)\left(2 m_{2}+1\right)\left(2 m_{2}+2\right)}\left[\delta_{r_{1}, m_{1}-1} \delta_{r_{2}, m_{2}+1}+\delta_{r_{1}, m_{2}+1} \delta_{r_{2}, m_{1}-1}\right] \\
& \left.+2 \sqrt{m_{1} m_{2}\left(2 m_{1}-1\right)\left(2 m_{2}-1\right)}\left[\delta_{r_{1}, m_{1}-1} \delta_{r_{2}, m_{2}-1}+\delta_{r_{1}, m_{2}-1} \delta_{r_{2}, m_{1}-1}\right]\right\} .
\end{aligned}
$$

In this representation $H_{k l}^{0}$ in (5.9) is not a diagonal matrix. As explained in $\S 4.2$ we need a diagonal $H_{k l}^{0}$ (from now on referred to as $D_{0}$ ). This is obtained by diagonalising $H_{k l}^{0}$ through the orthogonal transformation $U \cdot H_{k l}^{0} \cdot U^{-1}$ and by rotating $H_{k l}^{1}$ viz. $U^{-1} \cdot H_{1} \cdot U$. We mention here that Appendix B describes a simple method to calculate the matrix representation of $H_{1}$ in the basis of diagonal $H_{0}$. The difference with the aforementioned procedure is that knowledge of the basis where $H_{0}$ is diagonal is unnecessary and the calculations are carried out in infinite dimensional space and not in the truncated matrix space used above [KH 92a].

\subsection{Spectrum of the Quartic Oscillator}

To obtain the spectrum we used truncated matrices of dimension 1482 where $N=76$. An appropriate numerical routine is used to obtain the spectrum for $-1 \leq \lambda \leq 1$. The lower part of the spectrum is drawn in Fig. (5.4) where the known tendency to cluster in the region $\lambda=-1$ is apparent (higher up in the spectrum most of the 
Figure 5.4: Portion of the lower part of the spectrum for the quartic oscillator with $-1 \leq \lambda \leq 1$ on the horizontal axis. 
states are doublets). Owing to the truncation the higher energy levels are not accurate enough. Numerical studies (by varying the basis size) show that for $\lambda=0$ the first 450 levels have the required accuracy [SLK 88], [MWR 89]. This accuracy decreases with increasing $\lambda$ so that for $\lambda=1$ only the first 150 levels are stable.

The spectrum in Fig. (5.4) contains numerous avoided level crossings which are clearly discernible. The spectrum is a function of one parameter $\lambda$ only so that the avoided crossings will lead to exceptional points, and not diabolical points, in the complex $\lambda$-plane (see §4.3). The Hamiltonian matrix has time-reversal invariance and thus has co-dimension two. Due to the theorem by Von Neumann and Wigner discussed in $\S 4.3$, we conclude that there are no degeneracies in the spectrum. Accidental crossings can, however, occur. From our numerical studies described above we conclude that there are no accidental degeneracies in the studied range.

\subsection{Statistical Analysis}

Before the statistical analysis can be done, the spectrum has to be unfolded. This can be achieved by using the semiclassical limit method described in $\S(3.4)$. With this procedure one obtains the smoothed integrated level density [SLK 88], [Bo 92a]

$$
N_{a v}(E) \simeq C E^{\frac{3}{2}}
$$

where $C$ is calculated to be unity. When $\lambda=1$, however, the constant $C$ is infinite reflecting the classical unboundedness of this case. Simon proposed [Si 83]

$$
N_{a v}(E) \simeq C^{\prime} E^{\frac{3}{2}} \ln E .
$$

Tomsovic refined this expression to read [To 91]

$$
N_{a v}(E)=\frac{1}{2 \pi} E^{\frac{3}{2}} \ln E+\frac{E^{\frac{3}{2}}}{3 \pi}(2 \gamma+5 \ln 2-4)
$$

where $\gamma$ is Euler's constant.

\subsubsection{Spectral Statistics}

The rigidity of the spectrum has been investigated with the nearest neighbour spacing distribution and the $\bar{\Delta}_{3}$-statistic. These results are drawn in Figs. (5.5) and (5.6) respectively.

From the graphs it is evident that when $\lambda=0$ the system gives rise to Poisson statistics and the levels are strongly correlated revealing the classical integrability of the system. As $\lambda \rightarrow 1$ this correlation decays until the system is fully chaotic at $\lambda=1$. Similar results were obtained by Steeb et al [SLK 88] and Zimmermann et al [ZM 86]. The quantal system thus follows the behaviour of the classical system. 
Figure 5.5: The nearest neighbour spacing distribution for (a) $\lambda=-1$, (b) $\lambda=0$, (c) $\lambda=0.8$ and (d) $\lambda=1$ where a bin size of 0.28 was used. The dashed curves relate to the Poisson and GOE results.

Figure 5.6: The $\bar{\Delta}_{3}$-statistic (small circles) for (a) $\lambda=-1$, (b) $\lambda=0$, (c) $\lambda=0.8$ and (d) $\lambda=1$. The solid line and curve relate to the Poisson and GOE results. 
Figure 5.7: Average delocalisation length for the quartic oscillator. $l_{d}$ is shown for $-3 \leq \lambda \leq 1$.

From Figs. (5.5 a) and (5.6 a) we see that the system has intermediate correlations when $\lambda=-1$ with the nearest neighbour spacing distribution and $\bar{\Delta}_{3}$ curves lying between Poisson and GOE. This is evidence of the fact that the region $\lambda \in[-\infty,-1)$ can be mapped into the region $\lambda \in[-1,1]$ so that $\lambda=-\infty$ corresponds to $\lambda=1$. The reason for listing these redundant results will be discussed in $§ 5.7$.

\subsubsection{Eigenvector Statistics}

The average delocalisation lengths and distribution of individual delocalisation lengths of the eigenvectors have been calculated and are drawn in Figs. (5.7) and (5.8) respectively [HK 91]. These graphs show that the eigenvectors become more spread out as $\lambda \rightarrow 1$. But even at $\lambda=1$, where the classical system is fully chaotic, the eigenvectors are not delocalised as much as in the example discussed in $§ 4.6 .1$. This fact will be explained by the distribution of the exceptional points discussed in the next section. The eigenvectors for negative values of $\lambda$ are also delocalised although not fully delocalised showing intermediate correlations. The chaotic behaviour of the quartic oscillator thus also manifests itself in the behaviour of the eigenvectors.

\subsection{Exceptional Points}

We characterise the behaviour of a system by analysing the underlying mathematical mechanisms of the matrix operators $H_{k l}^{0}$ and $H_{k l}^{1}$ (see Chapter 4). This is done by studying the exceptional points of the mixed matrix problem given in (5.4). Application of the exceptional point formalism to this system is very interesting because this system is known to be regular at $\lambda=0$ (both classically and quantally) and then transforms into a fully chaotic system at $\lambda=1$. From our viewpoint, the classical 
Figure 5.8: The distribution of individual delocalisation lengths for (a) $\lambda=-1$, (b) $\lambda=0,(\mathrm{c}) \lambda=0.8$ and (d) $\lambda=1$.

counterpart is not of primary concern, yet it is instructive to compare our findings with the available knowledge of the problem.

To investigate the distribution of exceptional points, we calculated the line spectrum and the distribution of intersection points $P(N)$ [HK 91]. The system does not contain any degeneracies and (4.27) of $\S 4.5 .1$ can be used. The line spectrum is then given by

$$
E_{k}(\lambda)=\alpha_{k}+\lambda \omega_{k} ; \quad k=1,2, \ldots, M
$$

with $\alpha_{k}$ obtained as described in (4.26) and $\omega_{k}$ are the eigenvalues of $H_{1}$. The line spectrum for the quartic oscillator is drawn in Fig. (5.9) and the number distribution of the intersection points in Fig. (5.10). To see the correspondence between the line and actual spectrum, Fig. (5.11) shows both spectra for $N=4$. The two spectra coincide when $\lambda \rightarrow \pm \infty$.

For resolution purposes Fig. (5.9) is shown with $N=50$ only. Still it is clear that most intersections occur in the vicinity of $\lambda=1$. For small dimensions, Fig. (5.11) clearly shows that most avoided crossings in the actual spectrum and hence most crossings in the line spectrum occur near $\lambda=1$. This is substantiated by the number distribution drawn in Fig. (5.10) which distinctly shows a pronounced maximum at $\lambda=1$ (Here $N=76$ and $M=1482$ ). This is intuitively expected: the largest eigenvalues of $H_{k l}^{1}$ increase steadily with increasing $N$, hence the steepest (negative) slopes of the unperturbed lines increase with increasing $N$; further, the ratio of the 
Figure 5.9: Pattern of the unperturbed lines of the quartic oscillator. For the sake of clarity only a section of the spectrum for $N=76$ is shown.

Figure 5.10: Number distribution of intersection points of the unperturbed lines for the quartic oscillator. The normalisation is arbitrary. There are further intersections for $\lambda$-values beyond the range displayed. 
Figure 5.11: Actual spectrum (full lines) and line spectrum (dashed lines) for $N=4$ $(M=6)$. The similarity between the two spectra when $\lambda \rightarrow \pm \infty$ is evident.

largest eigenvalues of $H_{k l}^{0}$ to those of $H_{k l}^{1}$ tends towards unity with increasing $N$ which indicates an accumulation of intersection points at $\lambda=1$ with corresponding energy values near to zero. Intersection points beyond $\lambda=1$ are inherent to the finite dimensional matrix problem.

In $\S 5.3$ and $\S 5.6$ we showed respectively that the classical system tends more to a K-system and the quantum results tend more to the GOE results when $\lambda$ is increased from zero. Both the classical and quantum systems are known to be fully chaotic at $\lambda=1$. From Fig. (5.10) we see that the density of crossings also increases with increasing $\lambda$ up to its maximum at $\lambda=1$. Note that at $\lambda=0$ there are no intersection points and therefore no exceptional points expected (classical system integrable and quantal spectrum Poissonic). This is not visible due to the resolution of Fig. (5.10) but was confirmed by numerical studies. Fig. (5.10) thus shows that there is an accumulation of exceptional points in the region where chaos is discerned and that maximum chaos occurs at the maximum of the density of exceptional points.

From the spectrum in Fig. (5.4) and the line spectrum in Fig. (5.9) we note that there is less avoided crossings and hence less crossing points when $\lambda<0$ than when $\lambda>0$. This is also reflected in the density of crossings which shows that, although most exceptional points occur in the vicinity of $\lambda=1$, there is a considerable amount distributed over the whole allowable range where $\lambda<0$. This means that the exceptional points are not so densely distributed as those in the example discussed in 
§4.6.1. This is reflected in the statistical behaviour of the system. The $\bar{\Delta}_{3}$-statistic and nearest neighbour spacing distribution drawn in Figs. (5.6 a) and (5.5 a) respectively are between Poisson and GOE. It is also reflected in the delocalisation lengths of the eigenvectors drawn in Fig. (5.7). The eigenvectors for the quartic oscillator are not as delocalised as those of the example in $\S 4.6 .1$ and seem to resemble those of the example discussed in $\S 4.6 .2$ more.

From $\S 4.5 .3$ we, however, know that the coupling matrix elements are crucial in determining the behaviour of a system. The size of the coupling $C$ is given by the angles $\varphi$ that can be obtained from the orthogonal matrix $U$ that diagonalises $H_{k l}^{1}$ (see $\S 4.4 .4$ and $\S 4.4 .3$ ). The diagonal matrix $D_{1}$ can now be rotated to the original matrix $H_{k l}^{1}$ by increasing the angles from zero to their full values and thus transforming the line spectrum to the actual spectrum.

To see the effect of the coupling we note that the density of exceptional points (which corresponds to the density of states $d$ ) is rather small in the region where $\lambda<0$; the system is not fully chaotic. To evoke chaos in this region, the coupling matrix elements have to be enlarged so that the parameter $C \cdot d$ is large enough to ensure chaotic behaviour. This is accomplished by increasing the angles of $U$, for $\lambda<0$, by a factor of two. Note, however, that all contact with the classical problem is lost as the coupling matrix elements are now, on average, larger than the ones prescribed by the original problem. The effect of this operation fulfills all expectations: the emergent level statistics is in agreement with the GOE results and the delocalisation of the eigenvectors is enhanced.

The findings of this section is taken as evidence that the density of exceptional points is strongly correlated to the classical and quantal behaviour of the system. From this we conclude that the higher the density of exceptional points, the more likely chaotic behaviour is to occur. We now conjecture that the exceptional point formalism will provide the bridge that connects the classical and quantal behaviour of a system. The next section elaborates more on this point but it will be discussed in more detail in the next chapter.

\subsection{Periodic Orbit Analysis}

Eckhardt et al studied the periodic orbits and scarring in this system [EHP 89]. The densities $\left(|\Psi|^{2}\right)$ of some eigenfunctions for the $A_{1}$ symmetry class are plotted in Fig. (5.12). Here the potential energy contours are rotated by $\pi / 4$ so that the $\left(\tilde{q}_{1}, \tilde{q}_{2}\right)$ coordinates lie along the diagonals and $\lambda=0.99$. There are no nodal lines on the axes or the diagonals but it is obvious that many of the wave functions are localised in configuration space. These localisations are scars of unstable periodic orbits which are obtained from Gutzwiller's semiclassical analysis (see $\S 2.4$ ).

Eckhardt et al also obtained some unstable periodic orbits for this system and stated that for $\lambda=0.99$ all the periodic orbits that were found, were unstable. 
Figure 5.12: Densities $|\Psi|^{2}$ of some eigenstates in the $A_{1}$ symmetry class where $\lambda=$ 0.99. The upper number is the number of the eigenstate and the lower number a scaled energy (see [EHP 89]).

Figure 5.13: Unstable periodic orbits for the same eigenstates shown in Fig. (5.12) where $\lambda=0.99$ (from [EHP 89]). 
Dahlqvist and Russberg, however, showed that even when $\lambda=1$ there are some stable periodic orbits [DR 90]. Fig. (5.13) shows the orbits corresponding to the same energy as the eigenfunctions plotted in Fig. (5.12). A comparison of the orbits in Fig. (5.13) and the wave functions plotted in Fig. (5.12) shows that many of the wave functions have scars of unstable periodic orbits which is a feature of a quantum system having a classical chaotic counterpart [He 84].

The periodic orbits are due to an underlying periodic structure in the quantum chaotic spectrum [HC 92]. We conjecture that this underlying structure is obtainable through the exceptional point method and will be seen in the distribution of exceptional points. Preliminary results show that the origin of these unstable orbits and the scarring of the eigenfunctions around them can be understood through the exceptional point approach. This is discussed in more detail in the next chapter which elaborates more on the point that the exceptional points seem to be the natural quantities to help build the bridge between classical and quantum mechanics. The exceptional point formalism will thus help to obtain a sound mathematical description of the correspondence principle.

\subsection{Summary}

In this chapter the quantised version of a classical chaotic system was studied. The first two sections listed the classical results to show that when $\lambda=0$ the system is integrable and tends to a $\mathrm{K}$-system when $\lambda \rightarrow 1$. We also retrieved the known statistical results from the quantal spectrum: the system is Poissonic when $\lambda=0$ and becomes the more chaotic the larger $\lambda$. The $\bar{\Delta}_{3}$ statistic, nearest neighbour spacing distribution and delocalisation length of the eigenvectors all reproduce the GOE results for $\lambda \simeq 1$. Scarring of the eigenfunctions was also found to be in line with the results stated above.

By studying the density of intersection points we found that the density increases when $\lambda$ is increased from zero up to a maximum at $\lambda=1$. This thus follows the quantal and classical results and we conclude that there is a relation between the density of exceptional points (which is related to the density of states) and the behaviour of the system: the higher the density of exceptional points the more chaotic the system. The role of the coupling matrix elements was also discussed. We found that in regions where the density of exceptional points is low, chaos can be evoked by enlarging the coupling matrix elements. This is evidence of the fact stated by Guhr and Weidenmüller that the coupling and density of states are crucial quantities in characterising the behaviour of a chaotic system [GW 90]. Some preliminary and general arguments were given in relation to the idea that the exceptional points might be used to help obtain a mathematical description of the correspondence principle. 


\section{Chapter 6}

\section{The Hydrogen Atom in an Uniform Magnetic Field}

Since the discovery, by Zeeman in 1896, of the splitting of spectral lines when a light source was placed between the poles of an electromagnet, the study of the behaviour of atoms in the presence of a magnetic field was undertaken. This splitting is known as the linear Zeeman effect whilst the splitting of the spectral lines in strong magnetic fields is known as the Paschen-Back effect. In 1897 Lorentz developed the classical theory of the motion of an electron undergoing simple harmonic motion in the presence of a superimposed magnetic field.

The hydrogen atom is among the simplest bound state systems in nature that can be solved analytically. By imposing a magnetic field onto the system the behaviour and the solution of this simple system become more complex. For very strong fields the classical system is known as the diamagnetic Kepler problem which is nonintegrable. Quantum mechanically it also behaves chaotically. The conceptual importance of this problem follows from the fact that the two limiting cases of zero and infinite field strength (the Coulomb and the Landau problems respectively) which have very different symmetries, are the only three dimensional problems which are exactly soluble, classically and quantum mechanically. A thorough study of this system is interesting also because this model is a real physical system that can be studied experimentally. It was one of the first systems where quantum chaotic effects were seen in the laboratory. This has prompted many people to investigate this seemingly simple system. In undertaking a theoretical study of this system, we are thus doing real physics and are not just playing mathematical games.

\subsection{Introduction}

When the magnetic fields available in the laboratory are small, their action on the atom is well explained by perturbation theory. Some thirty years ago, however, 
astronomers found evidence showing that there are strong magnetic fields present in white dwarfs and extremely strong fields are assumed to exist in neutron stars [Ga 77]. This discovery led to laboratory experiments that imitated the extraordinary conditions in these stars in that strong magnetic fields are imposed on hydrogen atoms. Under these conditions the quadratic, or diamagnetic, term plays the dominant role and perturbation theory breaks down. This is known as the quadratic Zeeman effect. Classically the motion is chaotic and quantum mechanically it leads to GOE behaviour.

The Hamiltonian for the Hydrogen atom in a strong uniform magnetic field $\mathbf{B}=$ $B \hat{z}$ is (using atomic units and spherical coordinates)

$$
H=\frac{p^{2}}{2}-\frac{1}{r}+\frac{\gamma}{2} l_{z}+\frac{\gamma^{2}}{8} r^{2} \sin ^{2} \theta
$$

$H$ is derived in $\S$ D.1. Here $\gamma$ is a dimensionless parameter depicting the field strength $B$ viz.

$$
\gamma=\frac{B}{B_{0}} ; \quad B_{0} \simeq 2.35 \times 10^{5} \mathrm{~T}
$$

and $l_{z}$ is the $z$-component of the angular momentum. In this representation relativistic corrections to the simple model are negligible for fields with $\gamma<10^{4}$. In (6.1) the effects of spin-orbit coupling has been neglected which is permissible for strong fields where $\gamma n^{3}>10^{-4}$ with $n$ the principle quantum number and $\gamma n^{3}$ the ratio of the diamagnetic to the Coulomb energy [Ga 77].

In the first part of this chapter the classical Hamiltonian is scaled in such a way that the system is only dependent on one parameter, the scaled energy. Results from a classical Poincaré surface of section are then listed depicting the chaotic behaviour. The second part describes two methods to obtain a matrix representation and the results from a statistical analysis of the eigenvalues, showing GOE behaviour, are given. The third part is an in depth study into the exceptional point analysis of this system showing that the spectrum consists effectively of a set of curves which can easily be reproduced. Here we have a high density of exceptional points even in the nonchaotic regime. The fourth part looks at the role that the coupling matrix elements play in the behaviour of chaotic systems. This gives way to the definition of a measure that characterises the spectral behaviour of this system. This measure is only dependent on the coupling strength, the density of exceptional points and the interaction strength $\lambda$. The last part shows that results from the exceptional point formalism are consistent with those from a semiclassical analysis. We outline the mechanism which allows the periodic orbits to be retrieved with the exceptional point method. 


\subsection{The Classical System}

\subsubsection{Constants of Motion}

Classically the pure Coulomb problem has high symmetry, in fact seven constants of motion. For instance $H$ itself, the angular momentum vector $\mathbf{L}=\mathbf{r} \times \mathbf{p}$ and the Runge-Lenz vector [En 72]

$$
\mathbf{R}=-\frac{1}{2}(\mathbf{p} \times \mathbf{L}-\mathbf{L} \times \mathbf{p})-\frac{\mathbf{r}}{r} .
$$

The Coulomb system is called overintegrable since the scalar product $(-2 E)^{-1 / 2}(\mathbf{L}$. $\mathbf{R})=0$, in other words, the Runge-Lenz vector lies in the plane of the Kepler orbit. The symmetry group of the Hydrogen atom $(\gamma=0$ in $(6.1))$ is $S O(4)=S O(3) \otimes$ $S O(3)$. This leads to the high degeneracy when $\gamma=0$. For very large values of $\gamma$ a behaviour similar to a two dimensional oscillator prevails [DG 84].

\subsubsection{Scaling of the Hamiltonian}

In order to establish a connection between the classical and the quantal regime the structure of the classical trajectories in phase space has to be freed of all incidental features. Using cylindrical coordinates, $(\rho, \phi, z), H$ can be written (leaving out the term linear in $\gamma$ )

$$
H=\frac{p_{\rho}^{2}}{2}+\frac{p_{z}^{2}}{2}-\frac{1}{\sqrt{\rho^{2}+z^{2}}}+\frac{l_{z}^{2}}{2 \rho^{2}}+\frac{1}{8} \gamma^{2} \rho^{2} .
$$

The problem of classical motion has now been reduced to the study of orbits in a four dimensional phase space $\left(\rho, p_{\rho}, z, p_{z}\right)$. The classical equations of motion are invariant under the following transformations [Ro 81]

$$
\begin{aligned}
\tilde{\rho} & =\gamma^{2 / 3} \rho, \quad \tilde{z}=\gamma^{2 / 3} z, \quad \tilde{p}_{\rho}=\gamma^{-1 / 3} p_{\rho} \\
\tilde{p}_{z} & =\gamma^{-1 / 3} p_{z}, \quad \tilde{t}=\gamma t, \quad \tilde{l}_{z}=\gamma^{1 / 3} l_{z}
\end{aligned}
$$

and are governed by the scaled Hamiltonian

$$
\tilde{H}=\frac{\tilde{p}_{\rho}^{2}}{2}+\frac{\tilde{p}_{z}^{2}}{2}-\frac{1}{\sqrt{\tilde{\rho}^{2}+\tilde{z}^{2}}}+\frac{\tilde{l}_{z}^{2}}{2 \tilde{\rho}^{2}}+\frac{1}{8} \tilde{\rho}^{2}=H \gamma^{-2 / 3}
$$

which no longer depends explicitly on the field strength. The Hamiltonian in (6.4) shows that the classical dynamics depend on the scaled energy

$$
\varepsilon=E \gamma^{-2 / 3}
$$

only, and not on the energy $E$ and field strength $\gamma$ independently [WF 87]. The Hamiltonian (6.4) describes a point particle moving in the $\tilde{\rho} \cdot \tilde{z}$ plane in a nonseparable 
Figure 6.1: Equipotential lines of the potential in (6.6) in the $\tilde{\rho} \cdot \tilde{z}$ plane (from [FW 89]).

two dimensional potential consisting of the attractive Coulomb potential and the diamagnetic interaction where

$$
V(\tilde{\rho}, \tilde{z})=\frac{1}{8} \tilde{\rho}^{2}-\frac{1}{\sqrt{\tilde{\rho}^{2}+\tilde{z}^{2}}}
$$

which is shown in Fig. (6.1). Near the origin the potential is essentially the singular Coulomb potential and the trajectories resemble narrow Kepler ellipses.

\subsubsection{Classical Poincaré Surface of Section}

With the scaled energy in (6.5) and, $l_{z}=0$, the Poincaré surface of section can be obtained (for a review see [FW 89]). Use is made of semiparabolic coordinates

$$
\nu^{2}=\rho^{2}-z^{2}, \quad \mu^{2}=\rho^{2}+z^{2} .
$$

At a fixed scaled energy $\varepsilon$ the classical motion is confined to the energy shell, which is a three dimensional subspace of the four dimensional phase space spanned by the coordinates $(\nu, \mu)$ and the corresponding conjugate momenta $\left(p_{\nu}, p_{\mu}\right)$. This is shown in Fig. (6.2) with coordinates $\left(p_{\nu}, \nu\right)$.

For $\varepsilon=-0.8$ the motion is essentially regular (system almost integrable) and virtually all the trajectories lie on invariant tori. There are three fixed points visible from this section. The fixed points in the lower and upper parts of the section are independent of $\varepsilon$ and are located at $(0, \pm \sqrt{2})$. The separatrix separating these two regions of motion accumulates in a hyperbolic fixed point located at $( \pm \sqrt{2}, 0)$ for $\varepsilon=\infty$.

As the scaled energy is increased, irregular motion appears, first near the separatrix. It is clearly visible for $\varepsilon=-0.5$ and the separatrix is replaced by a stochastic layer which fills a finite region in the section. With a further increase, the separatrix layer increases in size until, for $\varepsilon=-0.1$, no regular structure is visible and the classical motion is dominated by global chaos. Similar results are obtained for the Lyaponov exponent [Fr 88], [Wi 87b]. 
Figure 6.2: Poincaré surface of section at $\varepsilon=-0.8,-0.5,-0.4,-0.3,-0.2,-0.1$ (from left to right and top to bottom [WH 89]). 


\subsection{The Eigenvalue Problem}

The azimuthal quantum number $m$, parity $\pi$ and (for high fields) $l_{z}$ are good quantum numbers. The underlying Hilbert space can therefore be divided into irreducible subspaces denoted by $m^{\pi}$ and we let $l_{z}=0$. For the remainder of this chapter the matrix representation will be obtained in the $m=0$ and positive parity subspace. We use the principle quantum number $n=1,2,3, \ldots, n_{\max }\left(n_{\max }\right.$ is the maximum $n$ considered) and the angular momentum quantum number $l=0,2,4, \ldots, n-1$. The Hamiltonian in (6.1) is now of the form

$$
H=H_{0}+\lambda H_{1}
$$

with (scaled by a factor 2)

$$
\begin{aligned}
H_{0} & =p^{2}-\frac{2}{r} \\
H_{1} & =\frac{1}{4} r^{2} \sin ^{2} \theta \\
\lambda & =\gamma^{2} .
\end{aligned}
$$

In (6.9) $H_{0}$ is just the expression for the ordinary Hydrogen atom, $\lambda$ is the dimensionless interaction parameter corresponding to the strength of the magnetic field $\mathbf{B}$ and $H_{1}$ describes the quadratic Zeeman effect. The Schrödinger equation reads

$$
\left[-\Delta-\frac{2}{r}+\frac{1}{4} \lambda r^{2} \sin ^{2} \theta\right] \psi=E \psi
$$

Attempts to solve the Schrödinger equation in (6.10) have a long history. Near the ionisation threshold the density of states and coupling strengths are high and a perturbative treatment ceases to be useful. To overcome this problem a group theoretical approach has been given by Delande and Gay [DG 84] while Clark and Taylor used Sturmian functions [CT 82]. At very high fields $(\lambda>1)$ a Landau basis set is practical [Fr 82]. For a review of these methods see Friedrich and Wintgen [FW 89], Garstang [Ga 77] and Taylor [Ta 90] and references therein.

$H$ in (6.8) is in a form appropriate to our investigations as outlined in $§ 4.2$. To characterise the behaviour we want to determine the exceptional points for this system. This is achieved through the line spectrum which is obtained from a matrix representation of $H$ with $H_{0}$ diagonal (see $\S 4.4 .3$ ). The most natural way of achieving this is to use the Coulomb basis set. This method is, however, not amenable to accurate numerical calculations. To overcome this problem Sturmian functions are then used. This leads to an $H_{0}$ which is not diagonal. The diagonalisation is then done numerically with the associated rotation of $H_{1}$. Both methods, using the Coulomb basis set and using Sturmian functions are described below. Only the Sturmian function method is, however, implemented throughout this chapter. The best method 
to compare the classical and quantum behaviour is due to Wintgen and Friedrich. They showed that the behaviour of the quantum system also depend on the scaled energy $\varepsilon$ introduced in $\S 6.2 .2$ only. This method is consequently used to investigate the statistical behaviour of the quantal spectrum for fixed values of $\varepsilon$ and is outlined in $\S \mathrm{D} .5$.

\subsubsection{The Coulomb Basis}

The Coulomb basis for the ordinary unperturbed Hydrogen atom in spherical coordinates is

$$
|n, l, m\rangle=R_{n l} Y_{l m}
$$

where

$$
R_{n, l}(r)=-\sqrt{\frac{(n-l-1) !}{2 n[(n+l) !]^{3}}\left(\frac{2}{n}\right)^{3}}\left(\frac{2 r}{n}\right)^{l} e^{-r / n} L_{n+l}^{2 l+1}\left(\frac{2 r}{n}\right)
$$

and

$$
Y_{l, m}(\theta, \phi)=(-1)^{m} \sqrt{\frac{(2 l+1)(l-m) !}{2(l+m) !}} P_{l}^{m}(\cos \theta) e^{i m \phi}
$$

with $Y_{l, m}$ known as the spherical harmonics. $L_{n+l}^{2 l+1}(2 r / n)$ are the generalised Laguerre and $P_{l}^{m}(\cos \theta)$ the generalised Legendre polynomials.

Using this basis leads to a $H_{0}$ which is a diagonal matrix given by

$$
\left\langle 0, l^{\prime}, n^{\prime}\left|H_{0}\right| n, l, 0\right\rangle=-\frac{1}{n^{2}} \delta_{l^{\prime}, l} \delta_{n^{\prime}, n}=E_{n}^{0}
$$

so that $E_{n}^{0}$ is $n / 2$ times degenerate for $n$ even and $(n+1) / 2$ for $n$ odd (recall: $m=0$ ). With the help of the Clebsch-Gordon coefficients a selection rule

$$
l^{\prime}=l, l \pm 2
$$

is obtained for $H_{1}$. The matrix representation for $H_{1}$ is given by

$$
\begin{aligned}
\left\langle 0, l^{\prime}, n^{\prime}\left|H_{1}\right| n, l, 0\right\rangle & =\frac{1}{4}\left\langle l^{\prime}, n^{\prime}\left|r^{2}\right| n, l\right\rangle\left\langle 0, l^{\prime}\left|\sin ^{2} \theta\right| l, 0\right\rangle \\
& =\frac{1}{4} \int R_{n^{\prime} l^{\prime}}^{*} r^{2} R_{n l} r^{2} d r \iint Y_{l^{\prime} 0}^{*} \sin ^{2} \theta Y_{l 0} d \Omega .
\end{aligned}
$$

with $d \Omega=\sin \theta d \theta d \phi$ the angular volume element. The angular integral is obtained viz. [BS 57]

$$
\begin{aligned}
\left\langle 0, l^{\prime}\left|\sin ^{2} \theta / 4\right| l, 0\right\rangle & =\frac{1}{8}\left\{\frac{2\left(l^{2}+l-1\right)}{(2 l+3)(2 l-1)} \delta_{l^{\prime}, l}-\right. \\
& -\left[\frac{l^{2}(l-1)^{2}}{(2 l-1)^{2}(2 l-3)(2 l+1)}\right]^{\frac{1}{2}} \delta_{l^{\prime}, l-2}
\end{aligned}
$$




$$
\left.-\left[\frac{(l+1)^{2}(l+2)^{2}}{(2 l+3)^{2}(2 l+1)(2 l+5)}\right]^{\frac{1}{2}} \delta_{l^{\prime}, l+2}\right\} .
$$

The radial integral $H_{r}=\left\langle l^{\prime}, n^{\prime}\left|r^{2}\right| n, l\right\rangle$ is evaluated with the help of Schrödinger's formula given in $\S($ D.2.2) equation (D.25) viz.

$$
\begin{aligned}
& H_{r}=\sqrt{\frac{(n-l-1) !}{(n+l) !}} \sqrt{\frac{\left(n^{\prime}-l^{\prime}-1\right) !}{\left(n^{\prime}+l^{\prime}\right) !}} \frac{1}{n n^{\prime}}\left(\frac{2}{n}\right)^{l+1}\left(\frac{2}{n^{\prime}}\right)^{l^{\prime}+1}\left(\frac{n n^{\prime}}{n+n^{\prime}}\right)^{5+l+l^{\prime}} \\
& \text { * }\left\{\sum_{\nu=0}^{n-l-1} \sum_{\mu=0}^{n^{\prime}-l^{\prime}-1}\left(4+l+l^{\prime}+\nu+\mu\right) ! \frac{(-1)^{\mu}}{\nu ! \mu !}\left(\frac{n^{\prime}-n}{n^{\prime}+n}\right)^{\nu+\mu}\right.
\end{aligned}
$$

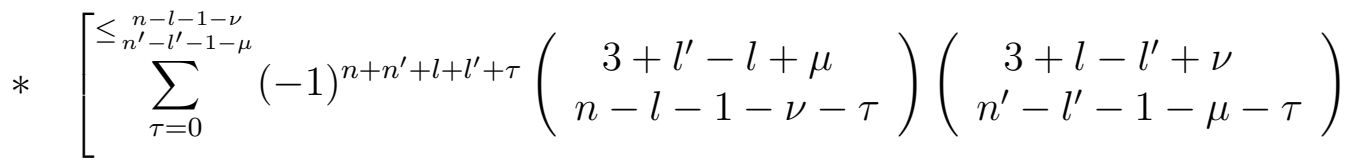

$$
\begin{aligned}
& \left.\left.* \quad\left(\begin{array}{c}
-\left(5+l^{\prime}+l+\mu+\nu\right) \\
\tau
\end{array}\right)\right]\right\}
\end{aligned}
$$

where

$$
\left(\begin{array}{l}
a \\
b
\end{array}\right)
$$

is a binomial coefficient. $\sum_{\tau=0}^{\leq_{p^{\prime}}^{p}}$ means the sum runs to the smaller of $p$ or $p^{\prime}$. From the binomial coefficients (or by applying the recurrence relation onto the Laguerre polynomial under the integral) the selection rule

$$
\left|n-n^{\prime}\right| \leq 3
$$

is deduced. The derivation of (6.18) is shown in $\S(\mathrm{D} .2 .2)$ and was evaluated by Schrödinger [Sc 26] and also used by Kupper [Ku 29].

When $n=n^{\prime}$ the radial integral contributes if $\nu=\mu=0$ so that the summation over $\nu$ and $\mu$ falls away to obtain

$$
\begin{aligned}
\left\langle n, l\left|r^{2}\right| n, l\right\rangle & =\frac{n^{2}}{2}\left[5 n^{2}+1-3 l(l+1)\right] \\
\left\langle n, l-2\left|r^{2}\right| n, l\right\rangle & =\frac{5 n^{2}}{2} \sqrt{\left[n^{2}-l^{2}\right]\left[n^{2}-(l-1)^{2}\right]} \\
\left\langle n, l+2\left|r^{2}\right| n, l\right\rangle & =\frac{5 n^{2}}{2} \sqrt{\left[n^{2}-(l+1)^{2}\right]\left[n^{2}-(l+2)^{2}\right]} .
\end{aligned}
$$

These equations were also deduced by Schiff and Snyder [SS 39].

Using (6.17) and (6.18) the matrix representation and corresponding spectrum for $H$ were calculated by Zimmerman et al [ZKK 80] and tested by Kotzé and Heiss [KH 92b]. Both found the method accurate for principle quantum number $n \leq 20$. 
While the diagonal terms $\left(n=n^{\prime}\right)$ for $H_{1}$ are easy to calculate, the off-diagonal terms, as depicted in (6.18), are not amenable to numerical implementation for $n \geq 21$. The reason being differences of the huge factorials and binomial coefficients under the summations of $\nu, \mu$ and $\tau$ which lead to accuracy problems (even when extended accuracy is used). This basis is also undesirable because it only takes the discrete states of hydrogen into account which is only permissible for weak magnetic fields.

\subsubsection{Sturmian Functions}

Sturmian functions were introduced by Rotenberg [Ro 70] and first applied by Edmonds [Ed 73] to the hydrogenic problem (see Appendix (D)). Although the eigenfunctions of the Schrödinger equation form a complete set, the fact that the continuum forms part of that set often reduces the completeness property to formal importance only. The continuum terms are then usually ignored because the ensuing equations are impossible to deal with. This is the case with the discrete Coulomb basis described in the previous subsection. This basis is incomplete in the sense that the positive energy of the atom (the continuum part) has been ignored. Sturmian functions, on the other hand, form a complete set of functions and can represent the Coulomb functions efficiently at small radial coordinate $r$. Clark and Taylor generalised the method of Edmonds in which case the Schrödinger equation can be written in the form of a generalised eigenvalue problem [CT 82].

Wunner et al proposed the following orthonormal and complete set of Sturmian functions as the radial eigenfunctions ${ }^{1}$ [WKR 86]

$$
G_{n l}^{(\zeta)}(r)=\zeta^{\frac{3}{2}} \sqrt{\frac{(n-l-1) !}{(n+l+1) !}} e^{-\zeta r / 2}[\zeta r]^{l} L_{n-l-1}^{2 l+2}(\zeta r)
$$

with $L_{n-l-1}^{2 l+2}(\zeta r)$ denoting the generalised Laguerre polynomials with $\zeta$ a positive real parameter. The basis for the Hamiltonian in (6.1) is now taken to be

$$
|n, l, 0\rangle=|n, l\rangle|l, 0\rangle=G_{n l} Y_{l 0}
$$

which is the most efficient approach close to the ionisation threshold [Ta 90]. The power of this basis lies in the freedom of choice for the parameter $\zeta$. $\zeta$ can be chosen to improve control over convergence and accuracy. It is appropriate to choose different values of $\zeta$ when examining different regions of the spectrum. Clark and Taylor showed that when choosing $\zeta$ such that

$$
\zeta=2 / n^{*} ; \quad n^{*}=1,2,3, \ldots, n_{\max }
$$

the eigenvalues are most accurate in the vicinity of $n^{*}$ [CT 82]. Valuable computing time is thus saved when extremely accurate eigenvalues have to be computed. The

\footnotetext{
${ }^{1}$ Wunner et al used the radial quantum number $n$ while, here, $n$ is the principle quantum number.
} 
range of accurate eigenvalues can be extended by choosing $n_{\max }$ considerably larger than $n^{*}$. The corresponding computing time and memory used are, however, also drastically increased.

\subsubsection{Matrix Representation with Sturmian Functions}

Using the basis in (6.21) leads to a representation of $H_{0}$ which is given by

$$
\begin{aligned}
\left\langle 0, l^{\prime}, n^{\prime}\left|H_{0}\right| n, l, 0\right\rangle & =-\frac{\zeta^{2}}{4} \delta_{n^{\prime}, n} \delta_{l^{\prime}, l} \\
& +\zeta^{2} \sqrt{\frac{(n-l-1) !}{(n+l+1) !}} \sqrt{\frac{\left(n^{\prime}-l^{\prime}-1\right) !}{\left(n^{\prime}+l^{\prime}+1\right) !}} \sum_{\tau=0}^{n-l-1} \frac{(2 l+\tau) !}{\tau !} \\
& *\left[(n-l-1)(n-l-\tau)\left(n^{\prime}-l-\tau\right)+(n-2 / \zeta)(2 l+\tau+1)\right. \\
& \left.-(n+l+1)(n-l-1-\tau)\left(n^{\prime}-l-\tau\right)\right] \delta_{l^{\prime}, l} .
\end{aligned}
$$

The matrix representation of $H_{0}$ is diagonal in $l$ but there is no restriction on $n$ or $n^{\prime}$. The radial integral using these generalised Laguerre polynomials is calculated in $\S$ D.2.1 (equation (D.21)) and (6.23) is derived in $\S$ D.4.2 (equation (D.47)) which is also given by Zeller [Ze 90]. Equation (6.23) can be evaluated numerically for large values of $n$ and $n^{\prime}$ because the terms under the summation are always of the same order.

The matrix elements for $H_{1}$ in the basis given by (6.21) can be expressed in closed analytical form. They give rise to a banded matrix. The selection rule in (6.15) is unaffected and matrix elements with respect to the radial functions in (6.20) vanish unless

$$
\left|n-n^{\prime}\right| \leq 2
$$

which can be obtained from the binomial coefficients in Schrödinger's radial integral. Using Schrödinger's radial integral, the radial matrix elements, $H_{r}=\left\langle l^{\prime}, n^{\prime}\left|r^{2}\right| n, l\right\rangle$, are found (see $\S$ D.4.1)):

When $l^{\prime}=l$

$$
\begin{array}{rll}
n^{\prime}=n-2 & : & H_{r}=\zeta^{-2} \sqrt{(n-l-2)(n-l-1)(n+l)(n+l+1)} \\
n^{\prime}=n-1 & : & H_{r}=-4 n \zeta^{-2} \sqrt{(n-l-1)(n+l+1)} \\
n^{\prime}=n & : & H_{r}=2 \zeta^{-2}[3 n(n+1)-l(l+2)] \\
n^{\prime}=n+1 & : & H_{r}=-4(n+1) \zeta^{-2} \sqrt{(n-l)(n+l+2)} \\
n^{\prime}=n+2 & : & H_{r}=\zeta^{-2} \sqrt{(n-l+1)(n-l)(n+l+2)(n+l+3)}
\end{array}
$$

and for $l^{\prime}=l+2$

$$
n^{\prime}=n-2 \quad: \quad H_{r}=\zeta^{-2} \sqrt{(n-l-1)(n-l-2)(n-l-3)(n-l-4)}
$$




$$
\begin{array}{rll}
n^{\prime}=n-1 & : & H_{r}=-4 \zeta^{-2} \sqrt{(n-l-1)(n-l-2)(n-l-3)(n+l+2)} \\
n^{\prime}=n & : & H_{r}=6 \zeta^{-2} \sqrt{(n-l-1)(n-l-2)(n+l+2)(n+l+3)} \\
n^{\prime}=n+1 & : & H_{r}=-4 \zeta^{-2} \sqrt{(n-l-1)(n+l+2)(n+l+3)(n+l+4)} \\
n^{\prime}=n+2 & : & H_{r}=\zeta^{-2} \sqrt{(n+l+2)(n+l+3)(n+l+4)(n+l+5)}
\end{array}
$$

and for $l^{\prime}=l-2$

$$
\begin{array}{rll}
n^{\prime}=n-2 & : \quad H_{r}=\zeta^{-2} \sqrt{(n+l-2)(n+l-1)(n+l)(n+l+1)} \\
n^{\prime}=n-1 & : \quad H_{r}=-4 \zeta^{-2} \sqrt{(n+l-1)(n+l)(n+l+1)(n-l)} \\
n^{\prime}=n & : \quad H_{r}=6 \zeta^{-2} \sqrt{(n+l)(n+l+1)(n-l)(n-l+1)} \\
n^{\prime}=n+1 & : \quad H_{r}=-4 \zeta^{-2} \sqrt{(n+l+1)(n-l)(n-l+1)(n-l+2)} \\
n^{\prime}=n+2 & : \quad H_{r}=\zeta^{-2} \sqrt{(n-l)(n-l+1)(n-l+2)(n-l+3)} .
\end{array}
$$

These equations are easy to implement numerically.

The $H_{0}$ and $H_{1}$ matrices can be constructed in different ways. Zeller ordered the quantum numbers as follows [Ze 90]

$$
\begin{aligned}
(n, l) \equiv & (1,0),(2,0),(3,0), \ldots,\left(n_{\max }, 0\right), \\
& (3,2),(4,2),(5,2), \ldots,\left(n_{\max }, 2\right), \\
& (5,2),(6,2),(7,2), \ldots,\left(n_{\max }, 2\right), \\
& \vdots \\
& \left(n_{\max }-1, n_{\max }-3\right),\left(n_{\max }, n_{\max }-3\right), \\
& \left(n_{\max }, n_{\max }-1\right) .
\end{aligned}
$$

This representation leads to an $H_{0}$ which is in block diagonal form and the underlying space is then reducible into irreducible $l$-subspaces. $H_{1}$ has block matrices on the diagonal and second diagonal only because $l^{\prime}=l \pm 2$. Each block matrix is banded according to $\left|n^{\prime}-n\right| \leq 2$.

For calculating the line spectrum, and determining the density of exceptional points (see §6.5.2), the following order is appropriate for $(n, l)$ (also see the discussion at the end of this section)

$$
(1,0),(2,0),(3,0),(3,2),(4,0),(4,2),(5,0),(5,2),(5,4), \ldots,\left(n_{\max }, n_{\max }-1\right) .
$$

Reasons for the preference of this order become obvious in $\S 6.5$. Using this order will lead to a representation where $H_{0}$ is a full matrix. The size of the elements, however, decrease on moving further away from the diagonal. $H_{1}$ is in semiblock form where the rows and columns consist of blocks which are indexed by $n=1,2, \ldots, n_{\max }$ and 
$n^{\prime}=1,2, \ldots, n_{\max }$ respectively. Taking the selection rule $\left|n^{\prime}-n\right| \leq 2$ into account will lead to an $H_{1}$ in the following form

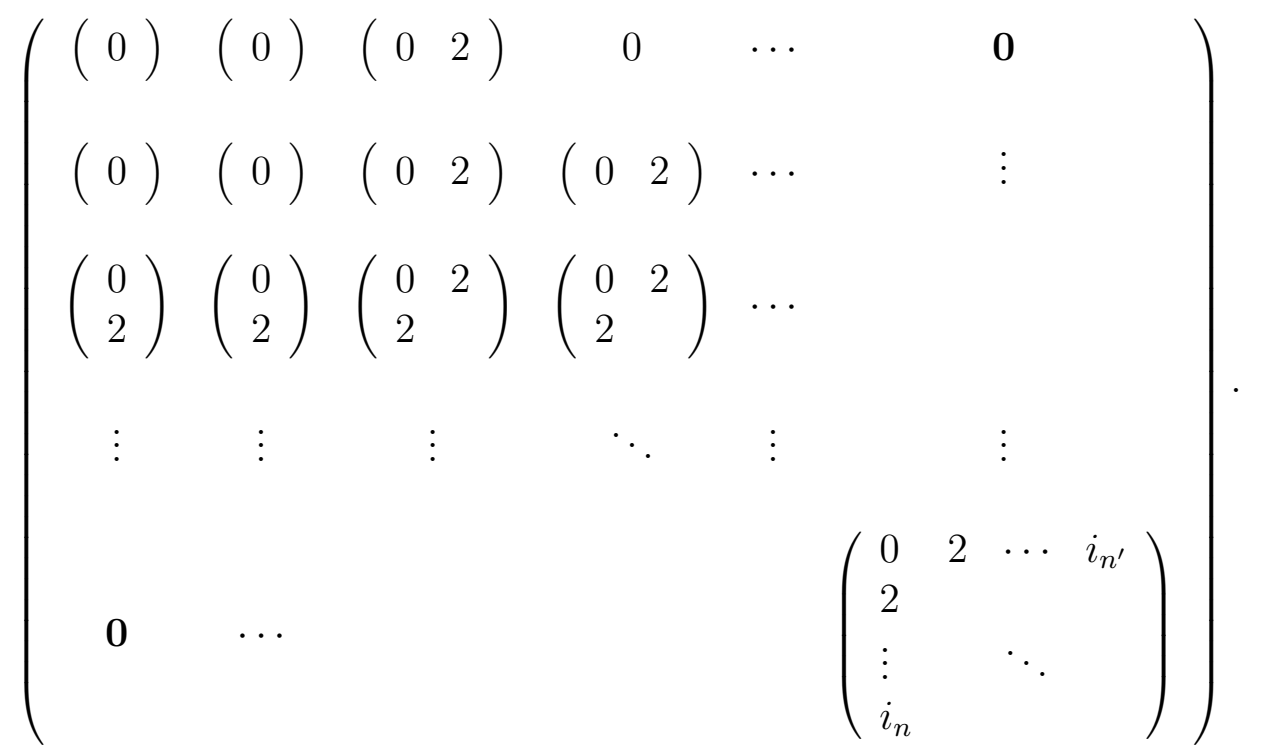

Each block in (6.29) is a square matrix

$$
\left(\begin{array}{lllll}
0 & 2 & 4 & \cdots & i_{n^{\prime}} \\
2 & & & & \\
4 & & & \\
\vdots & & & \ddots & \\
i_{n} & & & &
\end{array}\right)
$$

indexed by $\left(l, l^{\prime}\right)$ shown as the values for the rows and columns respectively where $i_{n}=n-1$ and $i_{n^{\prime}}=n^{\prime}-1$.

All calculations should be done in an infinite dimensional Hilbert space. For practical purposes, however, the space is truncated to the dimension of the matrix $H$ given by

$$
M=\left\{\begin{array}{l}
\frac{n_{\max }}{2}\left(\frac{n_{\max }}{2}+1\right) ; \quad n_{\max } \text { even } \\
\left(\frac{1}{2}\left(n_{\max }+1\right)\right)^{2} ; n_{\max } \text { odd. }
\end{array}\right.
$$

The full matrix representation of $H=H_{0}+\lambda H_{1}$ is now

$$
\left\langle 0, l^{\prime}, n^{\prime}|H| n, l, 0\right\rangle=\left\langle 0, l^{\prime}, n^{\prime}\left|H_{0}\right| n, l, 0\right\rangle+\lambda\left\langle l^{\prime}, n^{\prime}\left|r^{2}\right| n, l\right\rangle\left\langle 0, l^{\prime}\left|\sin ^{2} \theta / 4\right| l, 0\right\rangle
$$

and can be constructed using (6.17), (6.23) and (6.25)-(6.27).

The order used for $(n, l)$ in $(6.28)$ is a reshuffling of the order generally used in the literature. This has been appropriately taken into account when truncating 
the matrices. We have tested our procedure against the procedures described in the literature by comparing the eigenvalues so obtained with those by Friedrich and Wintgen [FW 89], Delande and Gay [DG 86], Killingbeck [Ki 79] and Clark and Taylor [CT 82]. The eigenvalues were found to be very accurate under variation of the parameter $\zeta$. The eigenvalues of $H_{0}$ can be tested against the exact values given in (6.14) and was found to be extremely accurate under variation of $\zeta$ [KH 92b].

A part of the spectrum for this system is drawn in Fig. (6.3) for $n=34,35, \ldots, 42$ with energy measured in Rydbergs. Here $n_{\max }=50$ and $\zeta=2 / 40$ so that $H$ is a $650 \times 650$ matrix which can easily be evaluated and diagonalised on a minisuper computer. The strength parameter $\lambda$ is taken between 0 and $10^{-9}$ which corresponds to a field strength of between 0 and 7.43 Tesla. At $\lambda=0$ the degeneracies of the pure hydrogen atom are seen while the lines do not actually cross one another for $\lambda>0$ but they undergo avoided level crossings. Fig. (6.4) shows another part of the spectrum where avoided level crossings are clearly discernible [KH 92b]. Here the spectrum is drawn for the strength parameter $\lambda$ between $0.5 \times 10^{-8}$ and $0.13 \times 10^{-7}$ corresponding to magnetic fields of between 16.62 and 26.8 Tesla using $n_{\max }=50$ and $\zeta=2 / 46$. The spectrum shown here and that obtained by Delande and Gay using the group theoretical approach ${ }^{2}$ is identical.

\subsection{Statistical Analysis}

In calculating the $\bar{\Delta}_{3}$-statistic and the nearest neighbour spacings distribution, the quantal spectrum was obtained using the scaled energy method of Wintgen and Friedrich described in $\S$ D.5. The statistical results now only depend on the scaled energy $\varepsilon$ through (6.5) namely the statistical behaviour will be the same for fixed $\varepsilon$ even if $E$ and $\lambda$ are changed.

This system is not time reversal invariant and we therefore expect GUE rather than GOE fluctuations. Berry and Robnik, however, replaced the concept of time reversibility by a more general concept of antiunitary symmetries [BR 86]. Now, because $H$ in (6.3) is invariant under a combination of time reversal and reflection through the $(x, y)$-plane, GOE fluctuations would prevail [HW 89]. Fig (6.5) shows the nearest neighbour spacing distribution and $\bar{\Delta}_{3}$-statistic for a few values of $\varepsilon$. The transition from Poisson to GOE behaviour is seen to happen at $\varepsilon \approx-0.35$ so that the system is fully chaotic at $\varepsilon=-0.1$. In the transitional region (from a Poisson to a Wigner distribution) the histograms can be approximated by a Brody distribution (see §3.5.2). We also say that the system behaves more chaotically for $\varepsilon=-0.1$ than for $\varepsilon=-0.5$ in the sense that the statistical results resemble more the GOE results for the first than the latter case.

These results correspond to the classical behaviour seen through the Lyaponov exponent and Poincaré surface of section (see $\S 6.2$ ). The $\bar{\Delta}_{3}$-statistic is seen to deviate

\footnotetext{
${ }^{2}$ The spectrum of Delande and Gay was not printed by them but by Bohigas et al [BGS 86].
} 
Figure 6.3: The spectrum for the hydrogen atom in a uniform magnetic field. The part shown here has $n=34,35, \ldots, 42$ with energy measured in Rydbergs. 
Figure 6.4: Another part of the spectrum so that the avoided level crossings are discernible. Here $0.5 \times 10^{-8} \leq \lambda \leq 0.13 \times 10^{-7}$ and $-0.0006 \leq E \leq-0.0004$ Ryds. 
Figure 6.5: (a) Nearest neighbour spacings distribution for a few values of the scaled energy showing the transition from Poisson to GOE behaviour. The solid line is the Brody fit. (b) $\bar{\Delta}_{3}$-statistic for a few values of $\varepsilon$ (from [FW 89]).

from GOE behaviour for large values of $L$. This is due to unstable periodic orbits in the system and can be explained using Gutzwiller's semiclassical analysis (see §2.4.4) [FW 89], [Gu 90].

\subsection{Exceptional Point Analysis}

In order to characterise the behaviour of this system, we now determine the distribution of exceptional points as discussed in Chapter 4 [HK 91]. This is achieved by using the line spectrum which was discussed in $§ 4.5$. All calculations are done for constant scaled energy $\varepsilon$ which preserves the classical behaviour [KH 92b].

\subsubsection{Line Spectrum}

By scrutinising the spectrum in Fig. (6.3) globally we observe that $H_{0}$ has systematic degeneracies and it seems as though the whole spectrum consists of a set of curves originating at the eigenvalues of $H_{0}$. Note that at $\lambda=0$ the crossings are the actual degeneracies of $H_{0}$; all other crossings are avoided but only look like actual crossings because of the resolution used. By inspecting the spectrum for negative $\lambda$ values (which is not physical but can be done for our matrix problem) we conclude that the curvature of the curves is negative for all values of $\lambda$, in particular for the positive ones. From Fig. (6.3) it is also evident that the curves start to repel one another to an increasing extent with increasing $\lambda$. The mixing regime begins, up to a first order 
approximation, at values given by [WF 86]

$$
\lambda \approx \frac{16}{5 n^{7}} \text {. }
$$

Globally, the simplistic and structured form of the spectrum is expected since the symmetries relating to $H_{0}$ and $H_{1}$ impose a particular structure on the full problem $H_{0}+\lambda H_{1}$. Here we have $O(4)$ symmetry when $\lambda=0$ and symmetries relating to the two dimensional oscillator when $\lambda$ is very large (see $\S 6.2 .1$ ). When looking at the spectrum the structure prevailing in the vicinity of $\lambda=0$ can be discerned over a fairly large range of $\lambda$-values when the actual level repulsions are interpreted as level crossings. In other words, while the detailed structure of the spectrum looks rather complex, an underlying simple structure is discernible. This simple structure is depicted through the line spectrum and is used to characterise the behaviour through the distribution of exceptional points [KH 92b].

In order to calculate the line spectrum we transform to a representation with $H_{0}$ diagonal. In doing so we actually obtain numerically a representation in the Coulomb basis with features described in $\S 6.3 .1 . H_{1}$ is in semiblock form ${ }^{3}$ where the selection rule in (6.19), $\left|n-n^{\prime}\right| \leq 3$ holds. Because $H_{0}$ has systematic degeneracies we use the guidelines given in $\S 4.5 .2$. In $H_{0}$, each principle quantum number $n$ is degenerate in the angular momentum quantum number $l . H_{0}$ can thus be reduced into subspaces characterised by $n$ which we term as $n$-subspaces. This is seen from the spectrum in Fig. (6.3) where the eigenvalues for $H_{0}$ are denoted by the $n$-values only. The line spectrum is then determined by the formulae given in (4.27) and (4.29) which are the asymptotic approximations to the actual spectrum when $\lambda$ is very large and when $\lambda$ is very small viz.

$$
\begin{aligned}
& E_{k}=\alpha_{k}+\lambda \omega_{k} ; \quad \lambda \gg 1 \\
& E_{k}^{\prime}=\epsilon_{k}+\lambda \delta_{k} ; \quad \lambda \ll 1 .
\end{aligned}
$$

Here $\epsilon_{k}$ and $\omega_{k}$ are the eigenvalues of $H_{0}$ and $H_{1}$ respectively and $k=1,2, \ldots, M$. $\alpha_{k}$ are the expectation values of $H_{0}$ in the basis of diagonal $H_{1}$ (for an explanation see $\S 4.5 .1)$ and $\delta_{k}$ are obtained by diagonalising $H_{1}$ in the different $n$-subspaces.

The diagonal $H_{0}$ can be reduced into a total of $n_{\max } n$-subspaces. The dimension for each subspace is given by

$$
M_{n}=\left\{\begin{array}{lc}
\frac{1}{2}(n+1) ; & n \text { odd } \\
\frac{n}{2} ; & \quad n \text { even }
\end{array}\right.
$$

The $E$-axis intercepts for $E_{k}^{\prime}$ are thus grouped into $n_{\max }$ sets; one set for each subspace which is actually given by $\epsilon_{j}=-1 / n^{2}\left(j=1,2, \ldots, M_{n}\right)$. With each intercept $\epsilon_{j}$ (in

\footnotetext{
${ }^{3}$ This was tested and found to be correct numerically.
} 
a particular subspace) we associate a slope $\delta_{j}$ which is an eigenvalue of the block of $H_{1}$ where $n=n^{\prime} . E_{j}^{\prime}$ is then one bunch of levels originating on the $E$-axis as depicted in Fig. (4.4).

The full line spectrum, near the origin, is obtained by taking the direct sum of the different subspaces where $\epsilon_{k}$ are ordered ascendingly and $\delta_{k}$ are ordered as in (4.30). $E_{k}^{\prime}$ is depicted on the left side of Fig. (4.4) with $k=1,2, \ldots, M$ where $M$ is defined in (6.30) and $M=M_{1}+M_{2}+\cdots+M_{n_{\max }}$. The total line spectrum for the system is obtained by connecting the two asymptotic spectra $E_{k}$ and $E_{k}^{\prime}$ given by (6.32) and (6.33). The appropriate connection is obtained by noting the following: firstly, the individual lines in each $n$-subspace, do not intersect one another. Secondly, the order of the lines for increasing values of $\lambda$ is given by the fact that the top most line of the $n$-subspace crosses all the lines of the $(n+1)$-subspace except the top most one; the second top line crosses all the lines of the $(n+1)$-subspace except the second top and top most lines ... the first line of the $n$-subspace does not cross any lines of the $(n+1)$-subspace. If the $E_{k}^{\prime}$ and $E_{k}$ lines are numbered $1,2, \ldots, M$ from bottom to top, the order of the $E_{k}$ lines will be

$$
\begin{aligned}
& 1,2,3,5,7,10,13,17, \ldots,\left(M-M_{n_{\max }}+1\right), \\
& 4,6,8,11,14,18 \ldots,\left(M-M_{n_{\max }}+2\right), \\
& 9,12,15,19,23, \ldots,\left(M-M_{n_{\max }}+3\right), \\
& 16,20,24,29, \ldots,\left(M-M_{n_{\max }}+4\right), \\
& \vdots \\
& \begin{cases}M-M_{n_{\max }}, M & n \text { even } \\
M & n \text { odd. }\end{cases}
\end{aligned}
$$

The connection between $E_{k}$ and $E_{k}^{\prime}$ is now obtained by a heuristic algebraic equation $\left(\tilde{E}_{k}\right.$ for the line spectrum) of the following form [KH 92b]

$$
\tilde{E}_{k}(\lambda)=\alpha_{k}+\lambda \delta_{k}-\left[\left|\alpha_{k}-\epsilon_{k}\right|^{3 / 2}+\lambda^{3 / 2}\left|\delta_{k}-\omega_{k}\right|^{3 / 2}\right]^{2 / 3} .
$$

The value of the power viz. $2 / 3$ is found to fit empirically sufficiently well; a value up to $3 / 4$ would do just as well. Equation (6.34) is a suitable choice because it reduces to (6.32) and (6.33) when $\lambda \rightarrow \infty$ and $\lambda \rightarrow 0$ respectively, and it depends only on quantities that can be derived from $H_{0}$ and $H_{1}$ alone; in other words we only use $\alpha$, $\omega, \epsilon$ and $\delta$. By construction the curve spectrum coincides with the spectrum of the truncated matrix $H_{0}+\lambda H_{1}$ only asymptotically for $\lambda \ll 1$ and $\lambda \gg 1$. In particular, for $\lambda \gg 1$ it shares all deficiencies of a spectrum obtained numerically by truncation. In the region of interest, which is the middle region, the curve spectrum does not reproduce the details of the actual spectrum. However, it does reproduce the details of the distribution of the real parts of the exceptional points.

In order for (6.34) to give the appropriate line spectrum all the lines should have 
negative curvature meaning that the following inequalities should hold

$$
\alpha_{k}>\epsilon_{k} ; \quad \delta_{k}>\omega_{k}
$$

However, because $\alpha_{k}$ and $\epsilon_{k}$ are obtained by rotations of the same matrix $D_{0}$, both obey the same trace rules and (6.35) cannot hold globally. We, however, found from extensive numerical tests that the rule in (6.35) will always hold in the vicinity of $n^{*}$ as long as $n_{\max } \geq 2 n^{*}$. Figure (6.6) shows the line spectrum for the same window of the actual spectrum depicted in Fig. (6.3). The similarity between the actual and line spectrum is striking. Here $n_{\max }=90$ and $n^{*}=40$ which leads to a matrix of order $2070 \times 2070$ to be diagonalised. This procedure is very efficient because the diagonalisation has to be done only once to obtain the 4 quantities $\alpha, \epsilon, \omega$ and $\delta$ for a specific $n^{*}$.

The line spectrum will now be used to obtain the density of exceptional points. This is possible because of the arguments given in Chapter 4 that the distribution of crossing points in the line spectrum is statistically equivalent to the density of exceptional points. The actual crossings of the lines are easily calculated where the intersection of the $k$-th and $l$-th line is determined by solving

$$
\begin{aligned}
0 & =\left(\alpha_{k}-\alpha_{l}\right)+\lambda\left(\delta_{k}-\delta_{l}\right)-\left[\left|\alpha_{k}-\epsilon_{k}\right|^{3 / 2}+\lambda^{3 / 2}\left|\delta_{k}-\omega_{k}\right|^{3 / 2}\right]^{2 / 3} \\
& +\left[\left|\alpha_{l}-\epsilon_{l}\right|^{3 / 2}+\lambda^{3 / 2}\left|\delta_{l}-\omega_{l}\right|^{3 / 2}\right]^{2 / 3} ; \quad l>k=1,2, \ldots, M
\end{aligned}
$$

for $\lambda$ using a standard Newton-Raphson numerical procedure.

\subsubsection{Density of Exceptional Points}

Before a meaningful method to determine the density of crossing points is described, we note that the spectrum is a function of the scaled energy $\varepsilon$ and principle quantum number $n^{*}$ only where the following relations hold [WF 87]:

$$
E=-\left(n^{*}\right)^{-2}
$$

and the corresponding $\lambda$-value is

$$
\lambda=\left(\frac{\varepsilon}{E}\right)^{-3}=\left[-\varepsilon\left(n^{*}\right)^{2}\right]^{-3} .
$$

The lines of constant $\varepsilon$ are invariant lines and are shown in Fig. (6.7).

A suitable representation of the distribution of crossing points is now obtained by keeping $n^{*}$ fixed. The distribution is then calculated by choosing a "window" between two scaled energies $\varepsilon_{1}$ and $\varepsilon_{2}$ where the top and bottom energies are defined by setting $n_{1,2}^{*}=n^{*} \pm 2$ in (6.36). The left and right $\lambda^{\prime}$ 's are obtained by setting $\lambda_{1}=\left[-\varepsilon_{1}\left(n^{*}\right)^{2}\right]^{-3}$ and $\lambda_{2}=\left[-\varepsilon_{2}\left(n^{*}\right)^{2}\right]^{-3}$. Here we have $\lambda_{1}<\lambda_{2}$ and $\varepsilon_{2}<\varepsilon_{1}$. This is schematically shown as one "window" (dashed box) in Fig. (6.7). 
Figure 6.6: Line spectrum for the hydrogen atom in a magnetic field. For the sake of comparison the same $E$ and $\lambda$ values as that depicted in Fig. (6.3) are shown. 
Figure 6.7: Schematical representation of the "windows" (dashed rectangles) used to calculate the distribution of crossing points. The solid curves are those for constant $\varepsilon$ with values as indicated.

The number of crossing points in a "window" is then calculated and the distribution $P$ obtained by dividing the distance $\varepsilon_{2}-\varepsilon_{1}$ into a number of equal length bins. The area under the histogram, so obtained, is normalised to unity so that $P$ for different $n^{*}$-values can be related, thus allowing different "windows" to be compared. This eliminates the effect of the increase in the density of states when $\varepsilon$ is kept fixed and $n^{*}$ increased. Figure (6.8) depicts histograms of the density of crossings for $n^{*}=25$ and $n^{*}=35$ with $\varepsilon_{1}=-2.0$ and $\varepsilon_{2}=-0.8$. The similar form of the histograms for the different values of $n^{*}$ is evident. This holds for all values of $n^{*}$ where we have chosen $n_{\max }=90$. The normalised density of crossings, $P$, is zero for $\varepsilon<-2.0$ because the invariant lines lie before the $n$-mixing regime where there are no crossings. Once inside the $n$-mixing regime however, $P$ increases rapidly to a maximum value $P_{\max }$ and then decreases gradually showing that the number of crossings decreases with increasing $\varepsilon$.

To render a direct comparison between the quantal and classical results we took many overlapping "windows" $\left(n^{*}=15,16,17, \ldots, 45\right)$ and calculated the distribution in each (see Fig. (6.7)). The total distribution of crossings is now a function of both $\varepsilon_{2}-\varepsilon_{1}$ and $n^{*}$ i.e. $P=P\left(n^{*}, \varepsilon_{2}-\varepsilon_{1}\right)$. The total distribution of crossings is represented by a three-dimensional graph as shown in Fig. (6.9) with $\varepsilon_{1}=-0.5$ and $\varepsilon_{2}=-0.2$. Here we have the scaled energy on the $x$-axis, the principle quantum number denoted by $n^{*}$ on the $y$-axis and the $z$-axis denotes the normalised density of exceptional points $P$. Evident is the global decreasing nature of $P$ with increasing 
Figure 6.8: Histograms depicting the density of crossing points for $-2.0 \leq \varepsilon \leq-0.8$ and principle quantum numbers (a) $n^{*}=25$; and (b) $n^{*}=35$ using 40 bins for each. Note the similarity in form of the two histograms.

scaled energy. The contourgraph ${ }^{4}$ for Fig. (6.9) is shown in (6.10). The lines shown are constant $P$-lines, each with a different value for $P$.

From Figs. (6.9) and (6.10) two observations are evident [KH 92b]: firstly, for fixed $n^{*}$, the normalised density of crossings, $P$, decreases with increasing $\varepsilon$ and secondly, statistically, the normalised density of exceptional points does not depend on $n^{*}$ (and thus the energy $E$ ) or $\lambda$ but only on the scaled energy $\varepsilon$.

The first observation confirms the fact, evident from the spectrum in Fig. (6.3), that the density of states decreases if $n^{*}$ is fixed and $\varepsilon$ increased. From this evidence we conclude there is a relation between the density of states and density of crossings $P$. The precise theoretical relation is, however, not yet known. $P$ does not relate the fact that the density of states increases if $\varepsilon$ is kept fixed and $n^{*}$ is increased because of the normalisation procedure used. $P$ is actually only the density of crossings at $\varepsilon_{1}$ relative to the density at $\varepsilon_{2}$. The actual density of crossings $P^{\prime}\left(n^{*}\right)$, for $\varepsilon$ fixed, is plotted in Fig. (6.11) for $\varepsilon=-0.2$. This clearly shows that the larger $n^{*}$ the higher the density of crossing points. The solid line is a numerical least squares fit which has the form

$$
P_{\varepsilon}^{\prime}\left(n^{*}\right)=a n^{*}+b\left(n^{*}\right)^{2}+c\left(n^{*}\right)^{3}
$$

showing the leading term for the actual density of crossings to be cubic in $n^{*}$. As shown in Fig. (6.11), (6.38) holds for for all relevant values of $\varepsilon$.

The second observation is consistent with results from a classical and quantum mechanical analysis which reveal that the quantum and classical systems only depend on the scaled energy $\varepsilon$ [FW 89]. In using the density of crossing points we have thus confirmed the quantum statistical and classical results. This substantiates the

\footnotetext{
${ }^{4} \mathrm{~A}$ graph obtained by looking at a three dimensional graph from the top (a geographical map). The lines are contours, each depicting a different height or in this instance a different $P$ value.
} 
Figure 6.9: Three-dimensional graph depicting the density of crossing points $P$ as a function of $\varepsilon$ and $n^{*}$. Here we have the scaled energy $\varepsilon$ on the $x$-axis, the principle quantum number $n^{*}$ on the $y$-axis and $P$ on the $z$-axis. We note that the density of crossings declines with increasing $\varepsilon$. 
Figure 6.10: A geographical map (contourgraph) of Fig. (6.9) showing that the density of crossing points only depends on the scaled energy. The lines are lines of constant $P$ with the higher values on the left side of the graph. Evident is the declining nature of $P$ which is constant in $n^{*}$. 
Figure 6.11: Actual density of crossings $P^{\prime}$ as a function of $n^{*}$ shown for $\varepsilon=-0.2$. The solid line is a least squares fit given by $P_{\varepsilon}^{\prime}$. We listed $P_{\varepsilon}^{\prime}$ on the left side for a few values of $\varepsilon$.

conjecture made in Chapter 2 that the exceptional point formalism can be used to bridge the gap between the RMT results of the quantum system and the results from a classical analysis. Further evidence is given below.

From the results described above we conclude that the density of exceptional points is higher for $\varepsilon_{1}=-0.5$ than for $\varepsilon_{2}=-0.2$. However, we know that the system behaves more chaotically the larger $\varepsilon$. Chaos sets in at $\varepsilon \approx-0.35$ and the system is fully chaotic for $\varepsilon=-0.1$. This seems to contradict the conjecture made in Chapter 4 that the higher the density of exceptional points the more likely chaos is to prevail. From $\S 4.5 .3$ however, we know that there are two physical quantities that prescribe the behaviour of a system: the density of states and the coupling matrix elements. The density of crossings gives an indication of the density of states and the coupling matrix elements determine the positions of the exceptional points which in turn determine the fluctuation properties of the spectrum. Both quantities thus have to be investigated to get a full description of the behaviour. The coupling matrix elements are discussed in the next section.

\subsection{The Coupling Matrix Elements}

In this section we investigate the interplay between the coupling matrix elements and the actual avoided crossings which are related to the exceptional points (see §4.4.5) [Ko 93]. Much insight is gained by firstly discussing the simple two dimensional model 
where $n=1,2$. The generalisation to higher order will lead to a quantitative measure to characterise quantum chaos.

\subsubsection{The Two Dimensional Matrix Problem}

For the two dimensional case the eigenvalues of $H_{0}$ are denoted by $\epsilon_{1}$ and $\epsilon_{2}$. Because $H_{0}$ is diagonal, it is reducible into two $n$-subspaces both of dimension one. If $\lambda$ is very small we know from perturbation theory that the coupling between the different $n$-subspaces can be neglected up to a first order approximation. $H_{1}$ is then in block diagonal form and also reducible into two $n$-subspaces both with dimension one. The eigenvalue of $H_{1}$ in the first subspace is denoted by $\delta_{1}$ and that in the second subspace by $\delta_{2}$. From (6.29) it is evident that $H_{1}$ is of the form

$$
H_{1}=\left(\begin{array}{ll}
\delta_{1} & C \\
C & \delta_{2}
\end{array}\right)
$$

where $C$ denotes the coupling between the eigenvalues, of the different $n$-subspaces, $\delta_{1}$ and $\delta_{2}$. For problems with dimensions higher than two, a more general form of $H_{1}$ exists, namely

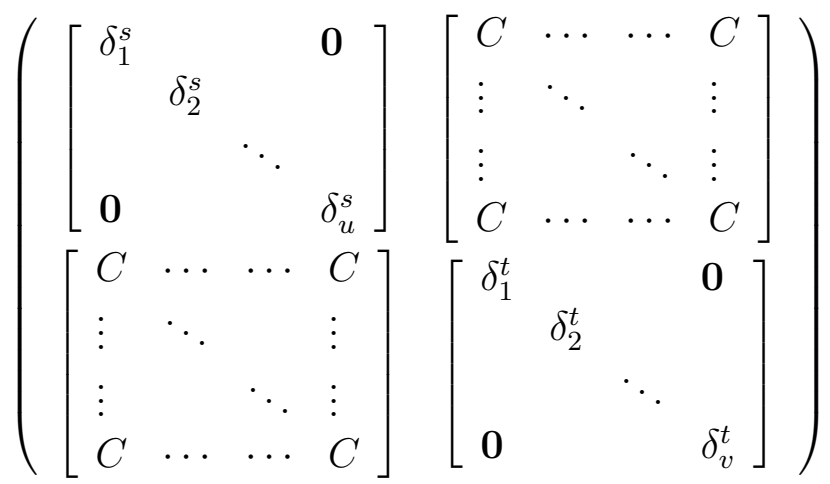

where $s$ and $t$ are adjacent subspaces. This matrix is equivalent to (6.39) if $u=v=1$. This specific form of $H_{1}$ will later be used to define an appropriate measure for the coupling strength.

Returning to the two dimensional case, the matrix representation of $H$ is

$$
H(\lambda)=\left(\begin{array}{cc}
\left(\epsilon_{1}+\lambda \delta_{1}\right) & \lambda C \\
\lambda C & \left(\epsilon_{2}+\lambda \delta_{2}\right)
\end{array}\right)
$$

with eigenvalues $\xi$ given by

$$
\xi_{1,2}=\frac{1}{2}\left[\left(\epsilon_{1}+\epsilon_{2}\right)+\lambda\left(\delta_{1}+\delta_{2}\right)\right] \pm \frac{1}{2} \sqrt{\left[\left(\epsilon_{2}-\epsilon_{1}\right)+\lambda\left(\delta_{2}-\delta_{1}\right)\right]^{2}+4 \lambda^{2} C^{2}} .
$$

The exceptional point for this two dimensional matrix $H$ is given at the $\lambda$-value where the discriminant vanishes (see $\S 4.4 .1$ and $\S 4.4 .3$ ) and

$$
\lambda_{c}=\frac{\epsilon_{2}-\epsilon_{1}}{\delta_{1}-\delta_{2} \pm 2 i C}=-\Delta \epsilon \frac{\Delta \delta \pm 2 i C}{\Delta \delta^{2}+4 C}
$$


where $\Delta \epsilon=\epsilon_{2}-\epsilon_{1}$ and $\Delta \delta=\delta_{2}-\delta_{1}$. If $C \ll \Delta \delta$ we have from (6.41)

$$
\Re \lambda_{c}=-\frac{\Delta \epsilon}{\Delta \delta} ; \quad \Im \lambda_{c}= \pm 2 C ; \quad \tan \varphi=\frac{2 C}{\Delta \delta} .
$$

From $\S 4.4 .3$ we know that to evoke chaos, the imaginary part of the exceptional point has to be appreciable in size. Equation (6.42) shows that small coupling will give a small $\Im \lambda_{c}$ and a small angle $\varphi$. The exceptional point thus lies close to the real axis and the avoided crossing is not appreciable. The two levels are too close to one another and the avoided level crossing is nearly an exact crossing which can not lead to chaotic behaviour (a situation also encountered in the example discussed in $§ 4.6 .2$ ).

To determine the effect of the coupling $C$ on the avoided crossing we determine the minimum of the spacing between the two levels which is given by (see $§ 4.4 .5$ )

$$
\Delta \xi=\xi_{2}-\xi_{1}=\sqrt{(\Delta \epsilon+\lambda \Delta \delta)^{2}+4 \lambda^{2} C^{2}} ; \quad \text { at } \lambda=\Re \lambda_{c} .
$$

By eliminating $\Delta \delta$ we obtain

$$
\Delta \xi=\left[\frac{\Delta \epsilon^{2}}{2}-\frac{\Delta \epsilon}{2} \sqrt{\Delta \epsilon^{2}-\left(4 \Re \lambda_{c} C\right)^{2}}\right]^{\frac{1}{2}} .
$$

Equation (6.43) shows that if $\Delta \epsilon$ and $C$ are both fixed, the spacing between the levels $\Delta \xi$ widens the larger $\Re \lambda_{c}$. Also, $\Re \lambda_{c}$ is fixed, the spacing can be enlarged by enlarging $C$. From these findings and the form of (6.43) we now conclude that the relevant parameter in determining the minimum spacing $\Delta \xi$ (of the avoided level crossing) is given by $\mathcal{B}$ such that

$$
\mathcal{B} \propto \lambda \cdot C
$$

If $\mathcal{B}$ is small we expect the avoided crossing to have a small gap (the levels nearly cross actually) and, if this happens on a large scale, to be outside the chaotic regime; in turn, for a larger value of $\mathcal{B}$ the gap size increases and the behaviour tends to be chaotic. These arguments are general and can also be applied to higher dimensions discussed in the next subsection.

\subsubsection{Generalisation to Higher Order}

For higher dimensional systems we note the following behaviour from (6.44) by keeping $\varepsilon$ fixed and by increasing $n$ (we move on the invariant line): firstly, let $\varepsilon$ be such that we are outside the chaotic regime ( $\varepsilon=-0.8$ for instance). When $n$ is small, $\lambda$ is large and the coupling has to be small to suppress the chaotic behaviour. This means that the imaginary part of $\lambda_{c}$ is very small and the exceptional points lie close to the real axis. The avoided level crossings are nearly actual crossings and no chaotic behaviour occurs. $\lambda$ decreases with increasing $n$ which now suppresses the chaotic 
behaviour. Under these circumstances the coupling $C$ may be larger without any significant effect on the behaviour. Secondly, if we keep $n$ fixed, we can move from the nonchaotic to the chaotic regime by increasing $\varepsilon$. This means that we effectively increase $\lambda$. This has the effect that even when $n$ and the coupling is small, $\lambda$ is can be large enough to ensure chaotic behaviour. This qualitative insight will be used in $\S 6.7$ to define a quantitative measure for the characterisation of chaotic behaviour.

To obtain a suitable measure of the coupling strength in the $M$-dimensional model, we apply the same arguments of the previous subsection. We transform $H_{1}$ to $H_{1}^{\prime}$ having the following form

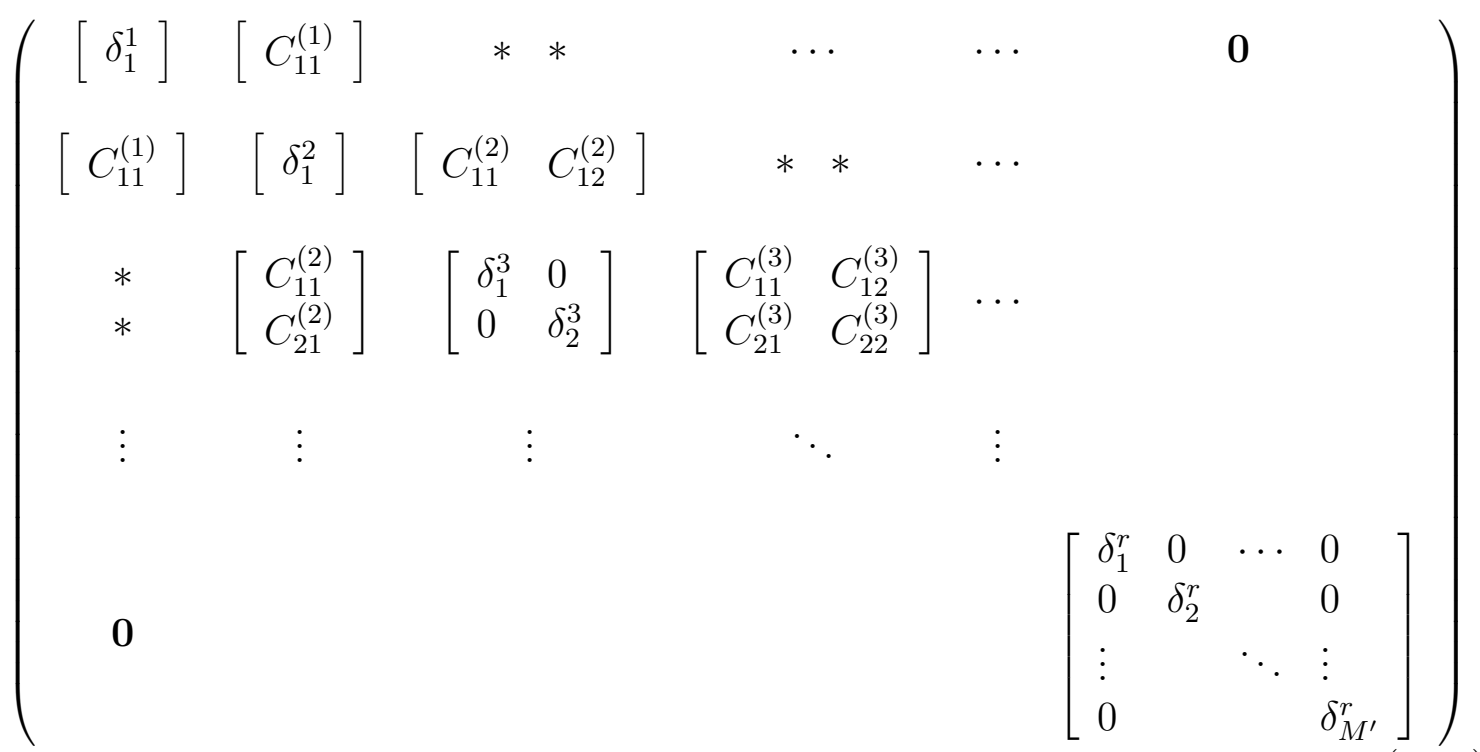

with $r=n_{\max }$ and $M^{\prime}=M_{n_{\max }}$ denoting the dimension of the last block. Each block on the diagonal is a diagonal $M_{n} \times M_{n}$ matrix with the eigenvalues for each $n$ subspace $\delta_{k}^{l}$ on the diagonal $\left(k=1,2, \ldots, M_{n}\right.$ denotes the different eigenvalues for each block and $l=1,2, \ldots, n_{\max }$ denotes the different $n$ subspaces). The blocks on the second diagonal are denoted by $C$ and the elements further out by asterisks. The off-diagonal elements are nonzero but tend to smaller values the further away from the diagonal. Here $C$ denotes the coupling between the different $\delta$ 's.

$H_{1}^{\prime}$ can be obtained through the following transformation

$$
H_{1}^{\prime}=U_{n_{\max }}^{-1} \cdots U_{2}^{-1} \cdot U_{1}^{-1} \cdot H_{1} \cdot U_{1} \cdot U_{2} \cdots U_{n_{\max }}
$$

where the different $U$ matrices commute with one another. $U_{k}$ is an orthogonal matrix 
such that

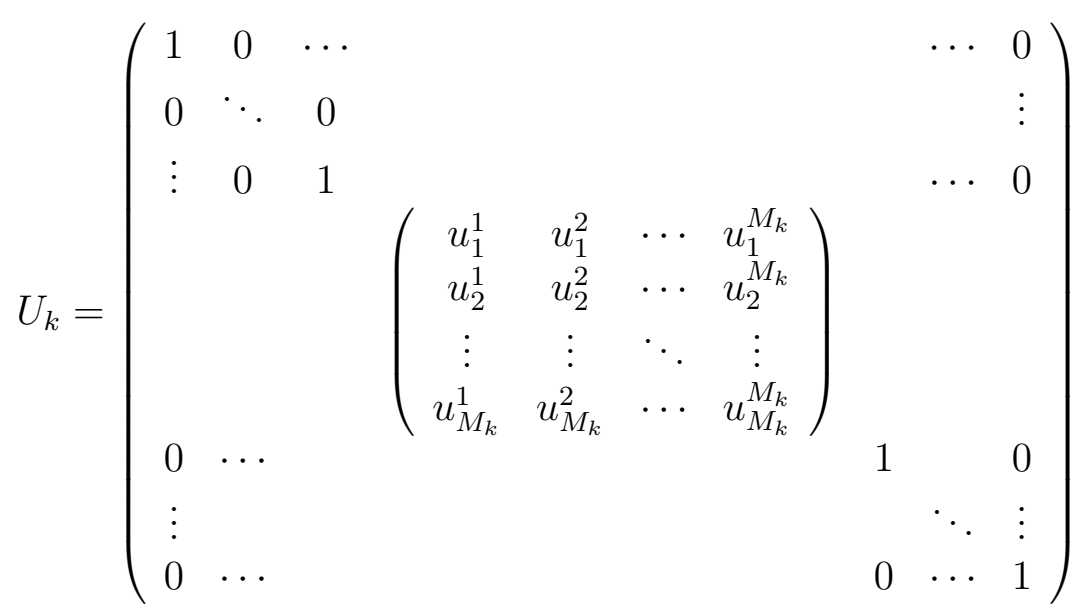

which is a unit matrix except for the matrix in place of the $k$-th block. The $k$-th block in (6.47) is the orthogonal matrix that diagonalises the $k$-th block in (6.29) with no $n$ coupling and $H_{1}$ rotated to the basis of diagonal $H_{0}$. We stress the fact here that $H_{1}^{\prime}$ can only be obtained if $H_{0}$ is diagonal and degenerate.

A quantitative measure for the strength of the coupling can be obtained if we assume that the $n$-coupling between individual elements of different blocks are small. $H_{1}^{\prime}$ are also in semiblock form and we only have to consider the coupling between the diagonal blocks as a whole. As a reasonable measure of the coupling between nearest neighbour diagonal blocks in (6.45) we use the norm of the off-diagonal block matrices viz.

$$
\bar{C}^{(i)}=\frac{1}{s r} \sum_{k=1}^{s} \sum_{l=1}^{r}\left|C_{k l}^{(i)}\right| ; \quad i=1,2, \ldots, n_{\max }-1
$$

with $s$ the number of rows and $r$ the number of columns in $C$. The coupling matrix elements as given by (6.48) are depicted in Fig. (6.12). Here $\bar{C}$ is drawn as a function of $n$ where $n_{\max }=90$ and $n^{*}=40$. Also plotted are the coupling due to the second off-diagonal blocks. They are much smaller than $\bar{C}$ and can thus be neglected. It is evident that the smaller $n$ the smaller the coupling. In using the definition given in (6.48) we also calculated the coupling between next nearest blocks on the diagonal. This coupling was found to be appreciably smaller than the nearest neighbour coupling justifying the assumption to neglect it.

In Fig. (6.12) $\bar{C}$ is fitted numerically by

$$
\bar{C}_{1}^{\prime}(n)=-0.0624 n-0.4889 n^{2}+0.2821 n^{3}
$$

and the leading term is cubic in $n$. The form of Fig. (6.12) and that of $H_{1}^{\prime}$ in (6.45) is now taken as evidence for the discussion given at the beginning of this section. Equation (6.49) shows that when $n$ is small, the coupling is so small that it can suppress the chaotic behaviour as characterised by $\mathcal{B}$ in (6.44). When $n$ is larger 
Figure 6.12: The coupling $\bar{C}$ as a function of $n$. The circles are the coupling due to the off-diagonal blocks and the diamonds due to the second off-diagonal blocks. The lines are numerical fits given by $\bar{C}_{1}^{\prime}$ and $\bar{C}_{2}^{\prime}$.

though, the coupling is larger but now $\lambda$ is smaller which then suppresses the chaotic behaviour.

The aforementioned characterisation does not yet explain the phenomenon that although we have a high density of exceptional points (for $\varepsilon=-0.8$ and large $n$ for instance) we still do not get chaos. This is outlined in the next section.

\subsection{A Quantitative Measure for Quantum Chaos}

In setting up a meaningful measure for the spectral behaviour of a system we take the results of the previous subsections into account. In (6.44) we found the behaviour of the two dimensional system to be proportional to $\lambda \cdot C$ [Ko 93]. This can be generalised to the $M$ dimensional case where we use $\bar{C}$ defined in (6.48) instead of $C$. We also know from $\S 4.5 .3$ that the density of states plays a crucial role and has to be incorporated into any measure of the behaviour. For the system under consideration we now propose the following quantity to be taken as a measure for the chaotic behaviour

$$
\mathcal{B} \propto \lambda \cdot \bar{C} \cdot d
$$

where $d$ denotes the density of states and $\bar{C}$ the coupling as defined in (6.48). Equation (6.50) measures the average gap between levels that cross in the complex E-plane i.e., the amount by which the levels repel one another. 
For $\mathcal{B}$ to be defined as an appropriate measure of the chaotic behaviour of a system, we have to analyse its properties. A desirable feature of any measure is that it should be dimensionless. This is the case for $\mathcal{B}$ defined in (6.50) because $\lambda$ is dimensionless, the unit of $\bar{C}$ is energy (Rydbergs) and that of $d$ is number of states per unit energy interval. We also want to describe the $n$-behaviour of $\mathcal{B}$. This is done by recalling from (6.37) that if $\varepsilon$ is kept fixed, $\lambda \sim n^{-6}$ and from (6.49) we have $\bar{C} \sim n^{3}$. The $n$-behaviour of $d$ is determined by considering its inverse, the mean level spacing. Let's consider an energy interval ranging from $n$ to $n+m$ and let $\bar{d}$ be the mean distance between the energy levels such that

$$
\begin{aligned}
\bar{d} & =\frac{\text { energy interval }}{\text { number of levels in interval }} \\
& =\frac{2}{m}\left[\frac{1 / n^{2}-1 /(n+m)^{2}}{n+(m+1) / 2}\right] .
\end{aligned}
$$

The leading term in (6.51) is $1 / n^{3}$ and hence the leading term for $d$ is $n^{3}$. From this and (6.50) it turns out that $\mathcal{B} \sim$ constant in $n$. We can thus put

$$
\mathcal{B}=\lambda \cdot \bar{C} \cdot d
$$

which is now taken as the appropriate measure for the behaviour of the system. We expect regular behaviour if $\mathcal{B}$ is small and chaotic behaviour if $\mathcal{B}$ has an appreciable value.

We however prefer to use the density of crossings rather than the density of states. From the results obtained in $\S 6.5 .2$ we conclude that there is a relation between the density of states and the density of exceptional points. The precise theoretical relation is not known. We have, however, established the leading term in the actual density of exceptional points $P^{\prime}$ to be $n^{3}$ as shown by (6.38) and Fig. (6.11). Now, by putting $d=P^{\prime}$ in (6.52) we have

$$
\mathcal{B}^{\prime}=\lambda \cdot \bar{C} \cdot P^{\prime}=\varepsilon^{-3} \cdot n^{-6} \cdot \bar{C} \cdot P^{\prime}
$$

which is also constant in $n$ if $\varepsilon$ is fixed. This is also reflected in the numerical analysis and the corresponding graph drawn in Fig. (6.13) which is a plot of $\mathcal{B}^{\prime}$ versus $n$ where we used a logarithmic scale on the vertical axis. Each line is obtained for a different value of $\varepsilon$ and is approximately constant in $n$. The larger $\varepsilon$ the more chaotically the system behaves which is reflected in the higher $\mathcal{B}^{\prime}$-values. The scaling has been chosen such that $\mathcal{B}^{\prime} \approx 1.0$ at $\varepsilon \approx-0.35$ which is the known scaled energy value where chaos sets in. $\mathcal{B}^{\prime}$ is thus a useful qualitative measure for the behaviour of this system which clearly shows that even though the density of exceptional points is high for certain small values of $\varepsilon$, the system does not behave chaotically. The reason is that the chaotic behaviour is suppressed by both small $\lambda$ and small coupling.

From the results stated above we now conjecture that a possible characterisation of quantum chaos hints at the following:

quantum chaos is a consequence of the interrelation between the coupling strength, the interaction strength and the density of exceptional points. 
Figure 6.13: The behaviour denoted by $\mathcal{B}^{\prime}$ as a function of $n$ where we use a logarithmic scale on the vertical axis. Each line corresponds to a different value of $\varepsilon$ as indicated with the corresponding $\mathcal{B}^{\prime}$ values listed on the right.

\subsection{Periodic Orbits and Exceptional Points}

Chapters 2 and 3 of this thesis described two formalisms for investigating quantal systems having classical chaotic counterparts: semiclassical mechanics and Random Matrix Theories. Hints at a possible link between the two methods were given in Chapters 2 and 5. The exceptional point methods seems to provide the mechanisms necessary for bridging the two formalisms. In this section we first mention results from a semiclassical analysis and then give preliminary arguments for using the exceptional point method in obtaining the semiclassical results. This is an interesting topic for continuing investigation.

Wintgen studied the periodic orbits using Gutzwiller's semiclassical periodic orbit theory showing that the semiclassical results also only depend on the scaled energy $\varepsilon$ [Wi 87a]. He found near exact agreement between the classical scaled actions and the positions of the peaks in the Fourier transform of the spectrum [WF 87]. Eckhardt and Wintgen calculated the form of the orbits making use of a symbolic coding scheme showing the chaotic features of this system to be directly related to the classical unstable orbits [EW 90]. Wintgen and Hönig also found the wave functions to scar around the unstable periodic orbits [WH 89] and Tanner et al showed how accurate quantum eigenvalues can be obtained in using Gutzwiller's trace formula [TS 91].

Holle et al studied the hydrogen atom in strong magnetic fields experimentally [HM 88] and found the spectrum so obtained in excellent agreement with the nu- 
merical calculated spectrum using (D.50) [WH 86]. They found the periodic orbits, obtained from classical trajectory calculations, to correlate with the resonances in the corresponding Fourier transformed spectrum [MW 86]. This shows that the origin of the chaotic features in the experimental spectrum is, therefore, directly related to the classical orbits.

The fact that results from a classical, semiclassical, quantum mechanical and exceptional point analysis all only depend on the scaled energy cannot be a coincidence since both the exceptional points and the periodic orbits relate to the substructure found in the spectrum and eigenfunctions of the system. This leads to the conjecture that we must be able to retrieve the periodic orbits and describe the mechanism of scarring by studying the exceptional points. On this point, let us briefly outline results of ongoing research.

Heiss and Chiang investigated a simple matrix model that mimics the quadratic Zeeman effect [HC 92]. They found that the systematic degeneracies (symmetries) in $H_{0}$ have a very characteristic effect on the spectrum of the full problem $H_{0}+\lambda H_{1}$. The periodic structure of the spectrum at $\lambda=0$ can be retrieved over the whole range of $\lambda$-values where chaotic behaviour is discerned. By investigating the eigenvectors they found definite localisation patterns in regions of the spectrum where there seems to be no obvious structure. This underlying structure of the spectrum can be related to the density of exceptional points. In regions of the spectrum where there are distinct groups of levels the density of exceptional points is high with strong coupling whereas the distribution is much more diluted between the groups and the coupling is less. This structure in the distribution of exceptional points is found throughout the chaotic spectrum. It is from this underlying structure that we believe that the periodic orbits can be retrieved.

There is even more structure in the spectrum of the hydrogen atom in a magnetic field in that there are scaling laws present. Structure was then also found in the distribution of exceptional points. We found in $§ 6.5 .2$ that the distribution of crossing points is similar for different $n$-values if the distribution is calculated between two fixed scaled energies $\varepsilon_{1}$ and $\varepsilon_{2}$. This just relates the underlying structure found in the spectrum. We must therefore be able to relate the structure found in the distribution of exceptional points to the periodic orbits. The precise theoretical relation is not yet known but the evidence stated above clearly indicates that such a relation exists. If this relation is found we conjecture that we will be able to understand the semiclassical results with the exceptional points formalism.

From Chapter 4 we know that the exceptional points determine the form of the spectrum and hence the fluctuation properties. The GOE results obtained from a statistical analysis is thus understood through the exceptional points. This and the last statement in the previous paragraph is then taken as evidence that the exceptional point approach forms a bridge between the semiclassical and RMT formalisms. 


\subsection{Summary}

From the results discussed above we have confirmed through our approach that the hydrogen atom in a strong uniform magnetic field shows all the features generally observed in quantal systems whose classical counterpart undergoes a transition from regularity to chaos as a certain parameter, $\varepsilon$ in this case, is changed.

It was also shown that the line spectrum, and hence the density of exceptional points, is easily obtainable from the eigenvalues and eigenvectors of $H_{0}$ and $H_{1}$ alone. The density of exceptional points do not depend on $\lambda$ and the energy $E$, but only on the scaled energy $\varepsilon$. We may have a high density of exceptional points even in the region of the spectrum where chaos is not discerned. The coupling matrix elements, however, play a crucial role in the behaviour of the system and has always to be taken into account. This relates to the angles of the rotation matrix $U$. When the density of exceptional points is small, the coupling matrix elements have to be large in order to obtain chaotic behaviour whilst for a large density of exceptional points, the coupling can be rather weak and chaotic behaviour can still prevail.

From the results obtained by studying the exceptional points and coupling matrix elements a measure $\mathcal{B}$ that characterises the behaviour was defined. The behaviour thus depends on the interplay between the coupling strength, the interaction strength and the density of exceptional points. This measure is easily calculated and is constant in $n$ if $\varepsilon$ is kept fixed which is consistent with the classical, semiclassical and quantum statistical results. From this we conjecture that the mechanism that produces scarring of the eigenfunctions and the underlying structure giving rise to the periodic orbits found from the spectrum can also be explained by the exceptional points. The last conjecture should generically apply to all chaotic systems which have classical chaotic counterparts.

The major aim of this chapter thus serves as a demonstration, that the exceptional points are relevant and that their distribution can be determined by a relatively inexpensive method.

$æ$ 


\section{Chapter 7}

\section{A One Dimensional Model for Quantum Chaos}

In the previous chapters, various systems showing features ascribed to 'Quantum Chaos' have been investigated. From a statistical analysis we concluded that the systems show a universal spectral behaviour. From an analysis of the quartic oscillator (Chapter 5) we found that the higher the density of exceptional points, the more likely it is that the system behaves chaotically. The hydrogen atom in a strong magnetic field showed the role of the coupling matrix elements in the actual positions of the exceptional points. In all the investigations the quantal systems had multidimensional classical chaotic counterparts. The following question now comes to mind: what if a system is purely quantal or one dimensional (or both) and still shows chaotic behaviour?

\subsection{Introduction}

By definition, all classical one dimensional systems are integrable and thus nonchaotic. Traditional quantum chaology investigations, on the other hand, require quantal systems that show chaotic behaviour in the semiclassical limit $(\hbar \rightarrow 0)$. This can then be rephrased by stating that all one dimensional quantal systems should not display chaotic behaviour. Quantum chaos is, however, so vaguely defined that it is compelling to ask the following question: can quantal systems, that are one dimensional, posses properties which are usually associated with quantised classical multidimensional nonintegrable systems? If so, another question arises: is quantum chaos a purely quantum mechanical phenomenon?

To elucidate these questions and attempt to answer them we propose a model of a single particle problem whose origin lies in a mathematically similar (though simpler) model [HK 88b]. It is a comparatively simple mathematical bound state model which combines a number of pertinent aspects relating to quantum chaos. The spectrum of 
this system shows a transition from a correlated to chaotic and back to a correlated spectrum. Although the spectrum is structured at both ends, the structures are very different in nature.

In the first part we introduce the system and then show that the spectra at both ends can be calculated algebraically. To investigate the intermediate spectrum the matrix Hamiltonian is calculated and diagonalised numerically. To elucidate the chaotic behaviour of this system, a proper statistical analysis is carried out on the eigenvalues and the eigenfunctions are also investigated. The second last part describes the behaviour of the expectation value of $x^{2}$ which also manifests the chaotic behaviour. The last part states some general comments on one dimensional chaotic systems.

\subsection{The Model}

We start with an infinite square well of unit extension in one dimension and put into it infinitely many $\delta$-function barriers on a Cantor set with uniform strength $\lambda$ [HS 89]. To construct the specific Cantor set $C$ used here, we define the open interval

$$
I_{s, t}=\left(\frac{3 t-2}{3^{s}}, \frac{3 t-1}{3^{s}}\right)
$$

and put

$$
G_{s}=\cup_{t=1}^{3^{s-1}} I_{s, t} \text { and } \quad G=\cup_{s=1}^{\infty} G_{s} .
$$

Here $s$ is the order and $t=1,2, \ldots, 3^{s-1}$. The set $C=[0,1] \backslash G$ is called the ternary Cantor set. It has cardinality $c$, is perfect, nowhere dense and has Lebesgue measure zero. The Hausdorff dimension and the capacity of $C$ assume the same value of $(\ln 2 / \ln 3)$.

The system is bounded from below and the potential for which the bound states are investigated is defined by

$$
V(x)= \begin{cases}\delta(x) & \text { if } x \in C \\ 0 & \text { if } x \in(0,1) \backslash C \\ \infty & \text { otherwise. }\end{cases}
$$

It appears that a Cantor set is an obvious mathematical device which allows infinitely many $\delta$-functions within a finite interval while preserving finite length of intervals between the $\delta$-functions. As we see below, these two conditions are crucial to obtain physically interesting patterns. We mention that the Cantor set yields a fractal structure in the partitioning of the unit interval. The model (shifted to the left by $1 / 2$ ), with order $s=3$, is drawn in Fig (7.1).

The Hamiltonian of the system is then in the form we used throughout this thesis (see $\S 4.2)$ i.e.

$$
H=H_{0}+\lambda H_{1}
$$




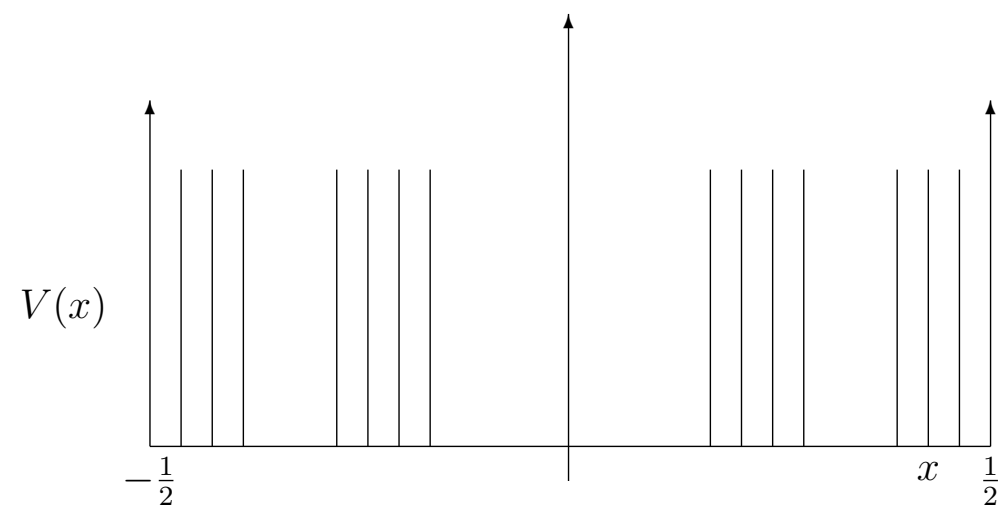

Figure 7.1: The square well with $\delta$-function barriers on a Cantor set, of order $s=3$, with uniform length $\lambda$. The symmetry around the origin is obvious.

with

$$
H_{0}=-\frac{1}{2} \frac{d^{2}}{d x^{2}} \text { and } H_{1}=V(x)
$$

and $\lambda$ is the strength (height) of the delta function barriers. $H_{1}=H_{\text {int }}$ is termed the interaction Hamiltonian and $\lambda$ the interaction strength.

\subsubsection{The Classical System}

The classical limit of this model is completely integrable. The potential barriers are infinitely high and the particle cannot penetrate them. The particle is classically bound between two $\delta$-function barriers (depending on the initial conditions) and the motion is fully periodic and independent of $\lambda$, except at $\lambda=0$.

\subsubsection{The Quantum Mechanical System}

If $\lambda<\infty$ the quantal particle will be able to penetrate the barrier potentials and it is scattered. Although there is no classical analogue of this system that behaves chaotically, we find for the quantised version a behaviour which is typical for quantum systems which have a classical counterpart with chaotic behaviour.

\subsection{The Spectrum}

Of interest here is the spectrum of $H$ as a function of $\lambda$. Since our interest is concerned with avoided level crossings, we have to reduce the problem to existing internal symmetries to obtain eventually an irreducible spectrum. The only symmetry in question is parity, and we focus our attention on even-parity solutions, where the wave function is symmetric with respect to the point at $x=0$. 


\subsubsection{Asymptotic Behaviour}

The model has the important feature that it gives rise to a transition from order to order at the two limiting cases where $\lambda=0$ and $\lambda \rightarrow \infty$. Obviously, the two cases are very different but the spectra of these two situations can be calculated analytically.

a) Case one: $\lambda=0$

For $\lambda=0$ the system reduces to a motion in the square-well potential and the spectrum is given by

$$
\frac{k_{n}}{\pi}=(2 n+1), \quad n=0,1,2, \ldots
$$

Here $k_{n}=\sqrt{E_{n}}$, where $E_{n}$ denotes the energy spectrum — the spectrum is totally rigid and harmonic.

\section{b) Case two: $\lambda \rightarrow \infty$}

For $\lambda \rightarrow \infty$ we encounter an infinite superposition of harmonic spectra, each being associated with square wells of extension $1 / 3^{s}, s=1,2,3, \ldots$ For a square-well between $[-1 / 2,1 / 2]$ we find

$$
\begin{array}{ll}
\frac{k_{n}^{(1)}}{\pi}=3(2 n+1) & \text { from the interval }\left[-\frac{1}{6}, \frac{1}{6}\right], \\
\frac{k_{n}^{(2)}}{\pi}=9(n+1) & \text { from }\left[-\frac{7}{18},-\frac{5}{18}\right] \text { and }\left[\frac{5}{18}, \frac{7}{18}\right]
\end{array}
$$

(sequence is 2 -fold degenerate),

$$
\begin{aligned}
& \frac{k_{n}^{(3)}}{\pi}=27(n+1) \quad \text { from }\left[-\frac{25}{54},-\frac{23}{54}\right], \ldots,\left[\frac{23}{54}, \frac{25}{54}\right] \\
& \text { (sequence is } 4 \text {-fold degenerate), } \\
& \frac{k_{n}^{(s)}}{\pi}=3^{s}(n+1) \quad \text { from }\left[\frac{2-3^{s}}{2 \cdot 3^{s}}, \frac{4-3^{s}}{2 \cdot 3^{s}}\right], \ldots,\left[\frac{3^{s}-4}{2 \cdot 3^{s}}, \frac{3^{s}-2}{2 \cdot 3^{s}}\right]
\end{aligned}
$$

(sequence is $2^{s-1}$-fold degenerate),

where $n=0,1,2, \ldots$. There is a $2^{s-1}$-fold degeneracy $(s=2,3, \ldots)$ relating to the $2^{s-1}$ intervals of length $1 / 3^{s}$. The spectrum is thus ordered but non-Poissonic which will be shown through the $\bar{\Delta}_{3}$-statistic obtained in $\S 7.4$. 


\subsubsection{The Intermediate Spectrum}

The previous subsection showed that the spectrum at $\lambda=0$ is harmonic and when $\lambda \rightarrow \infty$ it is highly ordered. The transition from the one order to the other is of particular interest.

There are two methods at our disposal to calculate the spectrum in this regime. The first one is to solve the Schrödinger equation directly and the second one is based on a matrix representation of $H$ and its diagonalisation. Both methods are described in Appendix E. The first method was used as a test whilst the latter method was used to obtain the spectrum for further investigations.

The matrix representation of the interaction Hamiltonian is given by (see $\S \mathrm{E} .2$ )

$$
\begin{aligned}
\left\langle m\left|H_{\text {int }}^{(+)}\right| n\right\rangle & =\lim _{s \rightarrow \infty} 2^{-s} \Re\left[Z_{s}(m+n)+Z_{s}(m-n)\right], \\
n, m & =1,3,5, \ldots
\end{aligned}
$$

where

$$
Z_{s}(q)=\exp \frac{i \pi q}{6}\left[\prod_{r=2}^{s}\left(1+\exp \frac{2 i \pi q}{3^{r}}\right)+\sum_{v=0}^{s-2} \prod_{r=2}^{s-v-1}\left(1+\exp \frac{2 i \pi q}{3^{r}}\right) \exp \frac{i \pi q}{3^{s-v}}\right] .
$$

For a numerical accuracy of $10^{-20}$ the maximum order of $s$ that can be used is given by (E.10)

$$
s_{\max }=44+\ln q .
$$

The full Hamiltonian matrix to be diagonalised is therefore

$$
H_{m, n}=m^{2} \pi^{2} \delta_{m, n}+\mu\left\langle m\left|H_{\text {int }}\right| n\right\rangle, m=1,3,5, \ldots
$$

with $\mu$ the effective interaction strength where $\lambda=\mu 2^{-s}$ so that $\lambda \rightarrow 0$ when $s \rightarrow \infty$ (see Appendix E). The spectrum is obtained as a function of $\mu$ by diagonalising $H_{n, m}$ in (7.6). The matrix is essentially sparse, yet the off-diagonal elements never tend to zero even when far away from the diagonal. The first 300 levels obtained from a $1486 \times 1486$ matrix were found to be numerically stable and are not affected by a truncation error. In Fig. (7.2) the lower part of the positive parity spectrum is illustrated. For a better visualisation we actually use the scaled eigenvalues $k_{n} / \pi$ with $k_{n}=\sqrt{E_{n}}$. For better demonstration we only show the first 80 levels. The emergence of the limit $\mu \rightarrow \infty$ is clearly discernible in the lower right part of Fig. (7.2).

The spectrum, as a whole, is characterised by many avoided level crossings. We stress that nowhere in the diagram do levels actually cross although they appear to do so due to the limited resolution. For demonstration we display in Fig. (7.3) a blown up version of the little box in Fig. (7.2). Because of the numerous avoided level crossings in the spectrum of this model, we conjecture that this model will behave chaotically. To get further insight into the behaviour of the system, the spectrum is analysed statistically. 
Figure 7.2: The first 80 energy levels versus the coupling constant $\mu$ ranging from 0 to 4000 . We actually have $k_{n} / \pi$ on the vertical axis. 
Figure 7.3: A blown-up version of the section in the little box in Fig. (7.2) showing the avoided level crossings.

\subsection{Statistical Analysis}

To analyse the spectrum we calculated $\bar{\Delta}_{3}(L)$ and $P(S)$, the nearest neighbour spacing distribution. Both quantities are obtained from the spectrum after unfolding. Since we do not have a phase space integral at our disposal, we have performed the unfolding by a best fit procedure. In Fig. (7.4) we display $\bar{\Delta}_{3}(L)$ and the corresponding nearest neighbour spacing distribution for a few parameter values. For intermediate values of $\mu$ the general trend seems to follow the GOE results manifesting the chaotic behaviour. The fact that the $\bar{\Delta}_{3}$ results lie above the GOE curve for large values of $L$ is indicative of the order that is emerging as $\mu \rightarrow \infty$.

At the two asymptotic values of $\mu$ we have a spectrum that is highly correlated. For $\mu=0$ the spectrum is rigid and harmonic with $\bar{\Delta}_{3}=1 / 12$. For $\mu \rightarrow \infty, \bar{\Delta}_{3}$ can be evaluated analytically using (3.22). Simple expressions for $\Delta_{3}$ are obtained if the $L$-interval ranges from $3^{m}$ to $3^{m^{\prime}}, m$ and $m^{\prime}$ being integers. For large $L$ of this kind the leading term in $\Delta_{3}(L)$ is given by $L^{2 \alpha} / 450, \alpha \approx \ln 2 / \ln 3$. It turns out that $\Delta_{3}$ oscillates between two envelopes where the lower envelope is given by the expression above while the upper one lies at values which are 10/3 times larger than those of the lower one. The line $L / 15$ relating to a Poisson spectrum lies slightly above the upper envelope for $L \leq 4406$ and between the two envelopes for $L \leq 437413$. When averages over initial $L$-intervals are taken, $\bar{\Delta}_{3}(L)$ comes close to the Poisson line for not too large values of $L$. Note, however, that $\Delta_{3}$ grows stronger than linearly which reflects the non-Poissonic nature of the spectrum for $\mu \rightarrow \infty$.

It is seen that at the two asymptotic values of $\mu$, the spectrum shows strong correlations whilst the intermediate spectrum shows intermediate correlations. Through these statistical results we see that there is a clearly discernible region, between the two ordered regions, where the system behaves chaotically. This is shown in Fig. 
Figure 7.4: (i) $\bar{\Delta}_{3}(L)$ (small circles) and (ii) nearest neighbour spacing distribution $P(S)$ for (a) $\mu=1000$ (b) $\mu=4000$ and (c) $\mu=10000$. The solid curves relate to the Poisson and GOE results. 
Figure 7.5: Schematic illustration of the regular and chaotic regions of the spectrum as a function of the interaction strength.

(7.5). From the results we now say that this one dimensional system displays features ascribed to quantum chaotic systems with a classical chaotic counterpart.

Evidence of another one dimensional quantum chaotic system was given by $\mathrm{Wu}$, Vallièrs and Feng [WV 90]. They studied a particle in a one dimensional potential so that the system is classically strictly integrable. By solving the Schrödinger equation they then searched for a potential whose spectrum will give rise to GOE statistics. Such a potential was shown to exist.

\subsection{The Wave Functions}

To get insight into the nature of the eigenfunctions, we expand a narrowly localised wave packet in terms of the eigenfunctions of the model Hamiltonian. We define the wave packet to be

$$
\psi(x)= \begin{cases}\sqrt{2 D} \cos D \pi\left(x-x_{0}\right) & \text { for } x \in\left[x_{0}-1 / 2 D, x_{0}+1 / 2 D\right] \\ 0 & \text { else }\end{cases}
$$

with width $1 / D$, and expand it as follows

$$
\psi(x)=\sum_{n}\left(c_{n} \Psi_{n}^{(+)}(x)+d_{n} \Psi_{n}^{(-)}(x)\right) .
$$

Here $\Psi_{n}^{(+)}$and $\Psi_{n}^{(-)}$are the positive and negative parity wave functions respectively. We calculate the expansion coefficients for a particle in the center interval $[-1 / 6,1 / 6]$. In Appendix E. 4 we show that this can be done for any interval and list the results for the interval $[5 / 18,7 / 18]$.

For the following discussions we have $x \in[-1 / 6,1 / 6]$ and let the wave functions be

$$
\begin{aligned}
& \Psi_{n}^{(+)}=N_{n} \cos k_{n} \pi x \\
& \Psi_{k}^{(-)}=M_{k} \sin \bar{k}_{k} \pi x
\end{aligned}
$$


where $N_{n}$ and $M_{k}$ are the normalisation constants (amplitudes) obtained from the eigenvectors, and $k_{n}$ and $\bar{k}_{k}$ are the wave numbers obtained from the eigenvalues. In order to get a description of the wave functions we expand them in terms of the ordinary square well bases (given in (E.4) and (E.5))

$$
\begin{aligned}
& |n\rangle^{(+)}=\sqrt{2} \cos n \pi x, \quad n \text { odd, (pos parity) } \\
& |k\rangle^{(-)}=\sqrt{2} \sin k \pi x, \quad k \text { even, (neg parity) }
\end{aligned}
$$

to obtain

$$
\begin{aligned}
& \Psi_{n}^{(+)}=\sum_{i=1}^{\infty} \alpha_{n}^{i}|n\rangle^{(+)} \\
& \Psi_{k}^{(-)}=\sum_{j=1}^{\infty} \beta_{k}^{j}|k\rangle^{(-)}
\end{aligned}
$$

where $\alpha_{n}^{i}$ and $\beta_{k}^{j}$ are the $n$-th and $k$-th positive and negative parity eigenvectors respectively. In order to get expressions for the normalisation constants, we calculate the derivatives of (7.12) and (7.13) and let $x=0$ so that

$$
\begin{aligned}
& N_{n}=\sqrt{2} \sum_{i} \alpha_{n}^{i}=\sqrt{2} X_{n} \\
& M_{k}=\frac{2 \sqrt{2}}{\bar{k}_{k}} \sum_{j} j \beta_{k}^{j}=\sqrt{2} Y_{k} .
\end{aligned}
$$

In Figs. (7.6) and (7.7) the amplitudes $X_{n}$ and corresponding power spectra are drawn for a few intermediate values of $\mu$. The normalisation is so chosen that $X_{n}=1$ if $\mu=0$ and $X_{n}=\sqrt{3}$ if $\mu \rightarrow \infty$ for those values of $\mathrm{n}$ where the eigenfunction makes a contribution within the center interval. Note the emergence of the large interaction limit at low values of $n$ with values of $X_{n}$ essentially at 0 and $\sqrt{3}$. The power spectra (see §3.6.2) are indicative for intermediate correlations in that they are continuous functions of the frequency, but not constants as it would be for a random set of amplitudes. The power spectrum is zero for $\mu=0$ and noisy when $\mu \rightarrow \infty$. We take this finding as further evidence that the one dimensional model considered here does behaves chaotically.

The coefficients in (7.8) are given by

$$
\begin{aligned}
c_{n} & =\frac{4}{\pi} \frac{X_{n}}{\sqrt{D}} \cos k_{n} \pi x_{0} \frac{\cos \frac{k_{n} \pi}{2 D}}{1-\left(\frac{k_{n}}{D}\right)^{2}} \\
d_{n} & =\frac{4}{\pi} \frac{Y_{n}}{\sqrt{D}} \cos \bar{k}_{n} \pi x_{0} \frac{\cos \frac{\bar{k}_{n} \pi}{2 D}}{1-\left(\frac{\bar{k}_{n}}{D}\right)^{2}} .
\end{aligned}
$$

According to these equations there is a smooth dependence on the parameters of the wave packet and we make the following observation: the dependence on the initial 
Figure 7.6: The amplitudes of the positive parity eigenfunctions in the center interval versus $n$ in the order of increasing eigenenergies. The cases for (a) $\mu=1000$, (b) $\mu=$ 4000 and (c) $\mu=10000$ are shown. 
Figure 7.7: The corresponding power spectra of the amplitudes shown in Fig. (7.6). The cases for (a) $\mu=1000$, (b) $\mu=4000$ and (c) $\mu=10000$ are shown. 
Figure 7.8: The autocorrelation coefficients of the amplitudes in Fig. (7.6) for (a) $\mu=4000$ and (b) $\mu=10000$. The coefficients decay exponentially.

conditions of the wave packet is smooth. The dependence of the coefficients $c_{n}$ and $d_{n}$ on $n$, however, looks rather erratic owing to the factors $X_{n}$ and $Y_{n}$. The term 'erratic' is used because of the form of autocorrelation coefficients which decays quite rapidly. The coefficients for a few values of $\mu$ are shown in Fig. (7.8).

The time evolution of the wave packet shows that it disintegrates faster than it would under the regime of a nonchaotic Hamiltonian [HK 90a]. This is brought about by what appears to be a general pattern in quantum chaos which is a tendency of the eigenfunctions to be localised in coordinate representation [CIS 88], [HT 83], [Fi 92]. The eigenfunctions of this system are essentially localised and the eigensystem seems to follow a pattern in that $\phi_{n}(x)$ contributes essentially only in a part of the domain and $\phi_{n+1}(x)$ in another. In this way the overlap $\left\langle\Psi \mid \phi_{n}\right\rangle$, using a localised wave packet $\Psi$, becomes an irregular function of $n$. This behaviour is reminiscent of scarring of eigenfunctions in two dimensional chaotic systems [He 84] but this is a formal analogy since no association can be made to periodic orbits.

\subsection{Expectation Value of $x^{2}$}

In order to get a better understanding of the concept of 'Quantum Chaos' every quantity that manifests the behaviour of a system is important. We conjecture that the behaviour of a system, must also manifests itself in the expectation value of $x^{2}$ i.e., through the spreading of the particle inside the wells. The results from such an investigation are actually more trustworthy because expectation values are independent of the reference state.

Using the wave functions in (7.9) and the amplitudes in (7.14) we can now calculate the expectation values of $x^{2}$ in the center interval (see Appendix E.5 for the full 
derivation). We obtained $\left\langle n\left|x^{2}\right| n\right\rangle$ in closed form to be

$$
\left\langle n\left|x^{2}\right| n\right\rangle=4 \begin{cases}\sum_{k=1}^{\infty} \sum_{l=1}^{\infty} \alpha_{n}^{k} \alpha_{n}^{l}\left\{\frac{4}{(a+b)^{3}(a-b)^{3}}\left[\sin \frac{a}{2} \sin \frac{b}{2}\left(\frac{a^{3} b}{2}-\frac{a b^{3}}{2}\right)\right]\right\} & a \neq b \\ \sum_{l=1}^{\infty} \alpha_{n}^{l} \alpha_{n}^{l}\left\{\frac{1}{48}-\frac{1}{8 a^{2}}\right\} & a=b\end{cases}
$$

where $a=\pi(2 k-1)$ and $b=\pi(2 l-1)$ and $\alpha_{n}^{l}$ is the $n$-th eigenvector of the matrix Hamiltonian in (7.6). The expectation values of $x^{2}$ are plotted in Fig. (7.9). Note the similarity with the form of the amplitudes plotted in Fig. (7.6). The power spectra and autocorrelation coefficients are therefore globally similar in nature to that plotted in Figs. (7.7) and (7.8). This clearly shows intermediate correlations for intermediate values of $\mu$ and strong correlations for $\mu=0$ and $\mu \rightarrow \infty$ [HK 90b].

\subsection{General Comment}

The potential of this one dimensional model is very complicated and the behaviour of the system might be ascribed to the 'freaky' potential. In more general terms though we know that any matrix representation of a Hamilton operator can be transformed into a tridiagonal matrix by successive orthogonal transformations; in turn, the eigenvalue equation of a tridiagonal matrix can be viewed as the discretised version of a second order differential equation which can be transformed into a discretised version of a one dimensional Schrödinger equation. From this general statement, the results obtained above for this one dimensional system and results from studies on multidimensional systems [He 88] the following conjecture is made: there must be an isomorphism between any multidimensional quantum model and a one dimensional system.

This transformation gives rise to a one dimensional variable which is not a usual space coordinate and the potential would also not be a usual well but will have a complicated structure like that of the model described in this chapter. This will then in turn give rise to complicated behaviour which is similar to GOE (or chaotic) behaviour as described in this chapter. This conjecture has actually been implemented for some models describing finite Fermi systems which can undergo phase transitions [HK 88b], [Sa 91]. Soft chaotic behaviour was detected in the transitional region.

Quantum chaotic behaviour has also been detected in another one dimensional system which does not have a classical counterpart. Jona-Lasinio and Presilla investigated a many-body system consisting of electrons undergoing multiple resonant tunneling [JP 92]. By using a mean field approximation they found the origin of the chaotic behaviour residing in the nonlinearity of the Hartree equation. $W u$ et al studied the quantum analogue of a classical integrable system and found that GOE 
Figure 7.9: Expectation values of $x^{2}$ for (a) $\mu=1000$, (b) $\mu=4000$ and (c) $\mu=10000$. 
level statistics can occur in such a system [WV 90]. This is a step closer in obtaining the link between the classical and quantum regimes which could be sought in the exceptional points of the system as put forward in the previous chapters. We conjecture that the periodic orbits of Wu's classical system can be retrieved by using the exceptional point method and we believe that it can also show how this system, while classically integrable, is quantally chaotic.

\subsection{Summary}

From the statistical analysis of this system we conclude that the spectrum shows intermediate correlations for intermediate interaction strengths whilst the two asymptotics show strong correlations. The spectrum shows numerous avoided level crossings i.e., exceptional points in the complex $\mu$-plane.

The behaviour of the coefficients $c_{n}$ and $d_{n}$ i.e. $X_{n}$ and $Y_{n}$, and that of the expectation value of $x^{2}$, shows the following character of the eigenfunctions:

- there is a decay of correlation amongst the individual state vector components,

- the eigenstates have a high sensitivity under perturbation and

- observations similar to scarring of the eigenfunctions are seen (no formal analogy to scarring can be made because this system does not have a classical counterpart).

This is in sharp contrast to the behaviour of the expansion coefficients relating to a regular Hamiltonian.

This chapter answered the first question asked in the introduction: from the behaviour of the eigenstates and the statistical analysis of the spectrum, we conclude that this one dimensional quantal system's behaviour is similar to systems showing GOE behaviour. Evidence of other one dimensional quantum chaotic systems that give GOE fluctuations was also given.

The second question asked in the introduction is more fundamental and needs more investigation. Although remote at this stage, one can argue that a detailed semiclassical investigation of one dimensional quantum chaotic systems, with classical counterparts, might shed light onto the origin of the quantum chaotic behaviour (like the system proposed by $W u$ et al [WV 90]). A periodic orbit analysis might supply satisfactory answers which can then be substantiated with a thorough exceptional point analysis. A semiclassical analysis of quantum chaotic systems has, at this stage, not been adequate enough to give a satisfactory explanation of the behavior of these quantum systems - a satisfactory connection between classical and quantum chaotic behaviour still evades us [Bo 92], [Gu 90]. The Gutzwiller trace formula is, for the time being, the only viable method for establishing such a connection (see $\S 2.4 .4$ ). The current system, however, does not have a classical analogue so that the 
trace formula is rendered useless. This gives more impetus to the conjecture that the origin of the chaotic behaviour should be sought in the distribution of the exceptional points which might serve to answer the second question. 
144 CHAPTER 7. A ONE DIMENSIONAL MODEL FOR QUANTUM CHAOS 


\section{Chapter 8}

\section{Summary and Conclusion}

In this thesis we address the following two questions: what are the common properties of the quantum mechanical operators that give rise to spectral properties that are ascribed to quantum chaos, and if a matrix representation of a Hamiltonian that originates from a classically chaotic analogy is given, what is the mathematical mechanism that yields the special features of the spectrum within the particular range where classical chaos is discerned?

From the fascinating study of quantum chaos, which remains at best vaguely defined, it is apparent that, quantum mechanical systems with classical analogues which display chaotic behaviour, show in corresponding regions universal patterns of spectral fluctuations. These fluctuations are statistically described with Random Matrix Theories where there is no physical input a priori. This raises a perplexing question: why do systems with no intrinsic statistical element such as those appropriately treated by semiclassical methods share universal fluctuation properties with matrices taken from a random Gaussian Orthogonal Ensemble? We conjecture that, the common denominator to answer these questions, irrespective of the physical origin of any system, lies within the exceptional points of the Hamiltonian. This, however, only addresses one aspect of the broader conjecture that the criteria for a definition of quantum chaos should be sought in the properties of the quantum mechanical operators.

Traditionally there are two methods to study quantum mechanical systems which have classical chaotic counterparts: the statistical approach using Random Matrix Theories and the semiclassical approach using the trace formula of Gutzwiller which relies on the remnants of the periodic orbits still detectable in the quantum system. In this thesis we introduce a third approach, the so-called exceptional point method. Indeed, in this work we probe the underlying mathematical structure of the quartic oscillator and the hydrogen atom in a strong uniform magnetic field (HASUMF) and investigate the fluctuation properties of a one dimensional quantum system which does not have a classical counterpart. The first two systems are known to be chaotic classically, semiclassically and quantum mechanically while the third shows a statistical 
behaviour similar to results from a GOE. This stands in contrast to the known fact that all one dimensional classical systems are integrable and therefore nonchaotic. Although no classical or semiclassical analysis was done on the systems under investigation, the known results are listed for comparison. The classical behaviour is manifested through the Poincaré surface of section and the Lyaponov exponent, the semiclassical behaviour through the scarring of the eigenfunctions and the unstable periodic orbits and the quantum mechanical behaviour through the $\bar{\Delta}_{3}$-statistic, the nearest neighbour spacing distribution and the delocalisation lengths of the eigenvectors.

Of course the definition of exceptional points rests upon the existence of a parameter that can be varied and thus classes of Hamiltonians of the form $H(\lambda)=H_{0}+\lambda H_{1}$ constitute an appropriate testing ground for our investigations. This seems a reasonable representative choice as most problems in quantum mechanics can be formulated by this Hamiltonian and in general it is a common starting point for studies in all branches of physics. The eigenvalues of $E_{n}(\lambda)$ of the Hamiltonian are the roots of the characteristic equation $\operatorname{det}[E-H(\lambda)]=0$ and the exceptional points are defined as the complex values of $\lambda$ for which two different eigenvalues coincide [HK 91]. For complex $\lambda$-values the operator is not selfadjoint thus excluding the genuine degeneracies of the selfadjoint problem as the eigenvalues do not coincide for any real $\lambda$-value.

If the two operators $H_{0}$ and $H_{1}$ do not commute, then the parameter $\lambda$ may serve to effect a transition from a nonchaotic to chaotic regime. In contrast to the classical case, the quantum mechanical transition from order to chaos is smooth, although it can be dramatic. Quantum chaos is associated with avoided level crossings (or tunneling) such that if they occur on a large scale then quantum chaos is likely to occur. This phenomenon is seen in all three systems that are discussed. On the other hand, exceptional points have a physical significance due to their relation with level repulsion. The connection is realised since the spectrum $E_{n}(\lambda), n=1,2, \ldots, N$ has branch point singularities at the exceptional points where two of the $N$ levels are joined via a square root branch point. If this occurs close to the real $\lambda$-axis, a level repulsion occurs for the two levels at real $\lambda$-values.

Strong correlations exists between the $N(N-1) / 2$ complex conjugate pairs of exceptional points and as a consequence, for sufficiently large $N$, the spectrum is affected by any perturbation of $H_{0}$ and $H_{1}$ [Sa 91]. The high sensitivity under generic perturbation of a chaotic spectrum and the corresponding state vectors is proposed as a signature of quantum chaos. Mathematical examples are used to highlight the relationship between the sensitivity to perturbation and the energy level density in the spectrum: the sensitivity depends on the ratio of the average coupling matrix elements and the mean level spacing. In an example where all the unperturbed levels cross at one particular value of $\lambda$, the mean level spacing is zero and hence the sensitivity to perturbation infinite (see $\S 4.6 .1$ ). This is bourne out by the very small angles required for the exceptional points to emerge from this point. Accordingly the eigenvectors show marked delocalisation, and the distribution of the localisation lengths the form 
associated with hard chaos, under this very slight perturbation. On the other hand, an unperturbed problem that has an extended region of level crossings where the average level spacing is finite and constant, requires a comparatively larger perturbation to obtain GOE-statistics (see §4.6.2). The eigenvectors are delocalised less strongly, but the delocalisation persists over the entire region and the distribution of delocalisation lengths has the form associated with soft chaos. The difference between the two examples can be understood in terms of the accumulation of exceptional points. In the first example the singularities are concentrated in a narrow window of the $\lambda$-range and due to the mutual influence of closely lying singularities, there is an enhanced global sensitivity to perturbation. In contrast, the exceptional points distribution is diluted in the the second example hence there is less mutual influence. Although local sensitivity to perturbation is retained, the overall global sensitivity is reduced and a larger set of angles is required to produce statistics obeying the Wigner surmise. This is substantiated through the density of crossing points and the delocalisation of the eigenvectors.

We have mentioned that it is impractical to determine the exact positions of the exceptional points but we have proposed a method by which the unperturbed lines of a spectrum may be determined by using knowledge from $H_{0}$ and $H_{1}$ alone. From the line spectrum the density of crossings (of the individual lines) can be obtained which is statistically equivalent to the density of exceptional points. From the analysis in this thesis we found that the statistical behaviour of a spectrum is highly correlated to the density of exceptional points. By analysing the quartic oscillator we found that the density of crossings follows the classical, semiclassical and quantum results. There is an accumulation of crossing points for the range of $\lambda$-values where the classical surface of section is nearly filled with points and only tiny regular islands prevail and where, semiclassically, scarring of the eigenfunctions around the unstable periodic orbits occur. The same holds in the region of the spectrum where quantum chaos is discerned and where the eigenvectors are delocalised. Maximal chaos occurs at the maximum density of crossings. We therefore propose that it is the distribution of exceptional points that give rise to the occurrence or absence of statistics ascribed to quantum chaos.

We further note that there are two physical quantities that prescribe the behaviour of a system: the density of states and the coupling matrix elements [GW 90]. The density of states is related to the density of exceptional points in that the higher the one, the higher the other (the precise theoretical relation is not yet known) and the size of the coupling is given by the angles $\varphi$ introduced in $\S 4.4 .3$ and $\S 4.4$.4. The effect of the coupling was seen in the quartic oscillator where chaos could be evoked in the system (in the nonchaotic regime where the density of crossings is low) by enlarging the angles $\varphi$. GOE statistics are obtained by making the coupling on average twice the size than that prescribed by the original problem.

Although we presume that it is generically the case, GOE type fluctuations must not necessarily occur if a high density of exceptional is found at a certain range of 
$\lambda$-values. In other words, in the example of $\S(4.6 .1)$ some specific nontrivial rotational matrices $U$ could possibly be construed which would produce spectra with little or no fluctuations for the spacings. However, the slightest generic perturbation of that situation would bring back GOE type fluctuations. It appears that the matrix $U$ in the quartic chaotic oscillator is sufficiently generic to generate the GOE type spectrum although there is no random element in it. What must be studied in this context is finding the (probably small) set of constellations of exceptional points which yields no fluctuations and determining the corresponding rotation matrices $U$ that produce such a constellation. From our experience we conjecture that this may be possible only for very specific spectra (like harmonic) of both operators, $H_{0}$ and $H_{1}$. We stress that it is not possible to prescribe a particular set of exceptional points since there are strong correlations between them.

The HASUMF has been described as the chaotic system par excellence because of its experimental tractability. This system was chosen to show that the exceptional point approach can be applied to real systems in nature and not just to mathematical models. The spectrum of the HASUMF is obtained by using Sturmian functions and has regions where the density of exceptional points is high, but the behaviour is nonchaotic. The line spectrum was obtained whose form is globally nearly exactly the same as that of the real spectrum. From the line spectrum we calculated the density of crossings that substantiated the fact that there are regions of a high density of states without chaotic behaviour. This shows that the coupling matrix elements play a very important role in characterising the behaviour [KH 92b].

The classical and quantum HASUMF can both be scaled to depend on one parameter, the scaled energy $\varepsilon$, only where

$$
\varepsilon=E \lambda^{-1 / 3}
$$

with $E$ the energy measured in Rydbergs. Lines of constant $\varepsilon$ are invariant lines whereon the chaotic behaviour is classically and quantum mechanically constant in principle quantum number $n$. By investigating the simple two dimensional matrix problem, we propose a measure which characterises the behaviour

$$
\mathcal{B}=\lambda \cdot \bar{C} \cdot P=\left(\varepsilon n^{2}\right)^{-3} \cdot \bar{C} \cdot P
$$

where $P$ is the density of crossings and $\bar{C}$ the average coupling matrix elements (see $\S 6.6 .2$ for the precise definition of $\bar{C}$ ). With $\varepsilon$ constant, $\mathcal{B}$ is constant in $n$ corresponding to the classical and quantum behaviour. The results thus show that the measure $\mathcal{B}$ follows the classical and quantum behaviour: $\mathcal{B}$ is small for the ranges of $\varepsilon$ where the system is nonchaotic (that is classically and quantum mechanically) but it increases dramatically when $\varepsilon$ is enlarged to take the system into the chaotic regime (the larger $\mathcal{B}$ the closer $\bar{\Delta}_{3}$ to GOE).

The semiclassical behaviour of the quartic oscillator and the HASUMF gives rise to another fundamental issue touched upon in this thesis: the substructure found 
in the spectrum and the eigenfunctions relating to the (unstable) periodic orbits of an associated classical system is believed to be related to a particular pattern of the exceptional points. This must be the case since the unstable periodic orbits are retrieved from hidden periodicities in the spectrum. These periodicities must reside within the distribution of the exceptional points. Preliminary results show that these periodicities can be seen in the distribution of crossing points in systems where $H_{0}$ has systematic degeneracies like the hydrogen atom. Scarring of the eigenfunctions also seems to have its origin through the sensitive dependence of the system on the exceptional points [HC 92]. The distribution of crossings of both the quartic oscillator and the HASUMF agrees with the results from a quantum mechanical and classical analysis. From this we conjecture that the exceptional point method provides the information necessary to build the bridge between the quantum and classical regimes. If the behaviour of the exceptional points is eventually understood in the limit of infinite dimensions, the exceptional point method may yield additional insight to obtain a mathematical description even of the correspondence principle. This statement needs a lot more attention and is the topic of continuing research using the work presented in this thesis as a basis.

Finally Chapter 7 describes a one dimensional model that shows a statistical behaviour similar to GOE. This is not the main theme of this thesis but is included because it serves to illustrate an interesting feature of quantum systems: while all one dimensional classical systems are integrable, one dimensional quantum systems can display chaotic behaviour. This behaviour was also seen in other one dimensional models. We thus conjecture that by investigating the exceptional points of seemingly simple one dimensional chaotic systems, which have classical counterparts that are integrable, can be instructive in understanding the behaviour of more complicated quantum chaotic systems.

To summarise: the spectra of systems with classical chaotic analogues contain many avoided level crossings which are associated with the exceptional points. The fluctuation properties of the spectra can be understood through the distribution of exceptional points where the exceptional point method probes the underlying mathematical structure of the spectrum. The unstable periodic orbits are due to periodicities in the spectrum. These periodicities must therefore also be retrievable from the distribution of the exceptional points. From the insight gained through the analysis of the exceptional points, we conjecture that quantum chaos is the more likely to occur the higher the density of exceptional points. Because of this and the fact that one dimensional systems can show chaotic behaviour, one might, now, try to give a characterisation of quantum chaos: quantum chaos is quantum mechanics under special conditions where there is a delicate interplay between the coupling matrix elements and the density of exceptional points. 


\section{Appendix A}

\section{Quartic Oscillator: Matrix Representation}

In order to obtain a matrix representation of the Hamiltonian in (5.1) e.g.

$$
H_{\alpha}(\mathbf{p}, \mathbf{q})=\frac{1}{2}\left(p_{1}^{2}+p_{2}^{2}\right)+V_{\alpha}(\mathbf{q})
$$

with

$$
V_{\alpha}(\mathbf{q})=\frac{1-\alpha}{12}\left(q_{1}^{4}+q_{2}^{4}\right)+\frac{1}{2} q_{1}^{2} q_{2}^{2} ; \quad \alpha \leq 1
$$

one has to change variables, scale the Hamiltonian and introduce Bose creation and annihilation operators. To avoid any degeneracies in the spectrum, group theory is used to obtain an irreducible representation of the Hamiltonian.

\section{A.1 Change of Variables}

The Hamiltonian in (A.1) is an Hermitian operator and can be transformed into a more appropriate form through the following change of variables ${ }^{1}$

$$
\begin{array}{ll}
q_{1}=\frac{1}{\sqrt{2}}\left(\tilde{q}_{1}+\tilde{q}_{2}\right) ; & q_{2}=\frac{1}{\sqrt{2}}\left(\tilde{q}_{1}-\tilde{q}_{2}\right) \\
p_{1}=\frac{1}{\sqrt{2}}\left(\tilde{p}_{1}+\tilde{p}_{2}\right) ; & p_{2}=\frac{1}{\sqrt{2}}\left(\tilde{p}_{1}-\tilde{p}_{2}\right) .
\end{array}
$$

With (A.3) substituted into (A.1) we obtain

$$
\tilde{H}\left(\tilde{p}_{1}, \tilde{p}_{2}, \tilde{q}_{1}, \tilde{q}_{2}\right)=\frac{1}{2}\left(\tilde{p}_{1}^{2}+\tilde{p}_{2}^{2}\right)+\frac{4-\alpha}{24}\left[\tilde{q}_{1}^{4}+\tilde{q}_{2}^{4}\right]-\frac{\alpha}{4} \tilde{q}_{1}^{2} \tilde{q}_{2}^{2}
$$

\footnotetext{
${ }^{1}$ This is a unitary transformation so that all the commutation relations are preserved.
} 
The Hamiltonian in (A.4) can now be scaled ${ }^{2}$ to facilitate various problems [Sh 88], [Si 70]. The underlying space is the infinite dimensional Hilbert space $L_{2}\left(\mathcal{R}^{2}\right)$ with eigenvalue equation (with $\hbar=m=1$ )

$$
\left[-\frac{1}{2}\left(\frac{\partial^{2}}{\partial \tilde{q}_{1}^{2}}+\frac{\partial^{2}}{\partial \tilde{q}_{2}^{2}}\right)+\frac{4-\alpha}{4}\left[\frac{1}{6}\left(\tilde{q}_{1}^{4}+\tilde{q}_{2}^{4}\right)\right]-\frac{\alpha}{4} \tilde{q}_{1}^{2} \tilde{q}_{2}^{2}\right] \psi_{m_{1} m_{2}}=E_{n} \psi_{m_{1} m_{2}} .
$$

Now let $(4-\alpha) / 4=\beta$ and with

$$
\begin{aligned}
\tilde{p}_{1} \rightarrow \lambda \tilde{p}_{1} ; & \tilde{p}_{2} \rightarrow \lambda \tilde{p}_{2} \\
\tilde{q}_{1} \rightarrow \lambda^{-1} \tilde{q}_{1} ; & \tilde{q}_{2} \rightarrow \lambda^{-1} \tilde{q}_{2}
\end{aligned}
$$

and $\lambda=\beta^{\frac{1}{6}}$, one obtains

$$
\left[-\frac{1}{2}\left(\frac{\partial^{2}}{\partial \tilde{q}_{1}^{2}}+\frac{\partial^{2}}{\partial \tilde{q}_{2}^{2}}\right)+\frac{1}{6}\left[\tilde{q}_{1}^{4}+\tilde{q}_{2}^{4}\right]-\frac{\lambda}{3} \tilde{q}_{1}^{2} \tilde{q}_{2}^{2}\right] \psi_{m_{1} m_{2}}=\epsilon_{n} \psi_{m_{1} m_{2}} .
$$

Equation (A.6) can be written as

$$
\tilde{H}^{\prime} \psi_{m_{1} m_{2}}=\epsilon_{n} \psi_{m_{1} m_{2}}
$$

where

$$
\epsilon_{n}=\sqrt[3]{\frac{4}{4-\alpha}} E_{n}
$$

and

$$
\lambda=\frac{3 \alpha}{4-\alpha}
$$

so that $-\infty \leq \lambda \leq 1$.

With $\tilde{H}^{\prime}$ denoted from now on by $\tilde{H}$ we have

$$
\tilde{H}\left(\tilde{p}_{1}, \tilde{p}_{2}, \tilde{q}_{1}, \tilde{q}_{2}\right)=\tilde{H}_{0}\left(\tilde{p}_{1}, \tilde{p}_{2}, \tilde{q}_{1}, \tilde{q}_{2}\right)+\lambda \tilde{H}_{1}\left(\tilde{q}_{1}, \tilde{q}_{2}\right)
$$

where

$$
\begin{aligned}
\tilde{H}_{0} & =\frac{1}{2}\left[\tilde{p}_{1}^{2}+\tilde{p}_{2}^{2}\right]+\frac{1}{6}\left[\tilde{q}_{1}^{4}+\tilde{q}_{2}^{4}\right] \\
\tilde{H}_{1} & =-\frac{1}{3} \tilde{q}_{1}^{2} \tilde{q}_{2}^{2} .
\end{aligned}
$$

An upper bound for the lowest eigenvalue can also be found

$$
\epsilon_{0}^{u b}=\left(\psi_{00}, \tilde{H} \psi_{00}\right)=\frac{3}{4}-\frac{\lambda}{8}
$$

where $($,$) denotes the inner product and \psi_{m_{1}, m_{2}}$ is the two dimensional Harmonic Oscillator basis

$$
\psi_{m_{1} m_{2}}=\left[2^{m_{1}+m_{2}} m_{1} ! m_{2} ! \pi\right]^{1 / 2} e^{\tilde{q}_{1} / 2} e^{\tilde{q}_{2} / 2} H_{m_{1}}\left(\tilde{q}_{1}\right) H_{m_{2}}\left(\tilde{q}_{2}\right) .
$$

Here $H_{m_{2}}\left(\tilde{q}_{2}\right)$ is the $m_{2}$-th Hermite polynomial so that $\psi_{m_{1} m_{2}}( \pm \infty)=0$ where $n, m_{1}, m_{2}=0,1,2, \ldots, N-1$ with $N$ the order of the system.

\footnotetext{
${ }^{2}$ The scaling was chosen so that the eigenvalues of $\tilde{H}^{\prime}$ (the scaled Hamiltonian) and $H_{\alpha}$ in (A.1) are equal when $\alpha=\lambda=0$.
} 


\section{A.2 Bose Operator Representation}

In order to obtain a matrix representation for $\tilde{H}$ in (A.9) we introduce Bose creation and annihilation operators defined as follows

$$
\begin{aligned}
b_{k}^{\dagger} & =\frac{1}{\sqrt{2}}\left(\tilde{q}_{k}-i \tilde{p}_{k}\right) \\
b_{k} & =\frac{1}{\sqrt{2}}\left(\tilde{q}_{k}+i \tilde{p}_{k}\right) ; \quad k=1,2 .
\end{aligned}
$$

The following commutation relation holds

$$
\left[b_{k}, b_{l}^{\dagger}\right]=\delta_{k l}
$$

and all other commutators are zero.

$\tilde{H}_{0}$ and $\tilde{H}_{1}$ are now written in terms of these operators viz.

$$
\begin{aligned}
\hat{H}_{0} & =\frac{1}{4}\left[\hat{n}_{1}^{2}+\hat{n}_{2}^{2}\right]+\frac{3}{4}\left[\hat{n}_{1}+\hat{n}_{2}\right]+\frac{1}{6}\left[b_{1}^{\dagger} b_{1}^{\dagger} \hat{n}_{1}+\hat{n}_{1} b_{1} b_{1}+b_{2}^{\dagger} b_{2}^{\dagger} \hat{n}_{2}+\hat{n}_{2} b_{2} b_{2}\right] \\
& +\frac{1}{24}\left[\left(b_{1}^{\dagger}\right)^{4}+b_{1}^{4}+\left(b_{2}^{\dagger}\right)^{4}+b_{2}^{4}\right]+\frac{3}{4}
\end{aligned}
$$

and

$$
\begin{aligned}
\hat{H}_{1}= & -\frac{1}{12}\left[b_{1}^{\dagger} b_{1}^{\dagger}+b_{1} b_{1}+b_{2}^{\dagger} b_{2}^{\dagger}+b_{2} b_{2}+b_{1}^{\dagger} b_{1}^{\dagger} b_{2}^{\dagger} b_{2}^{\dagger}+b_{1}^{\dagger} b_{1}^{\dagger} b_{2} b_{2}+b_{1} b_{1} b_{2}^{\dagger} b_{2}^{\dagger}+b_{1} b_{1} b_{2} b_{2}\right] \\
& -\frac{1}{6}\left[\hat{n}_{1}+\hat{n}_{2}+b_{1}^{\dagger} b_{1}^{\dagger} \hat{n}_{2}+b_{1} b_{1} \hat{n}_{2}+\hat{n}_{1} b_{2}^{\dagger} b_{2}^{\dagger}+\hat{n}_{1} b_{2} b_{2}\right]-\frac{1}{3}\left[\hat{n}_{1} \hat{n}_{2}+\frac{3}{4}\right] . \quad \text { (A.17) }
\end{aligned}
$$

$\hat{n}_{k}=b_{k}^{\dagger} b_{k}$ are the number operators.

\section{A.2.1 Matrix Representation of the full Hamiltonian}

The matrix representation of $\hat{H}=\hat{H}_{0}+\lambda \hat{H}_{1}$ is obtained by using the two dimensional oscillator basis (in number representation)

$$
\left|m_{1}, m_{2}\right\rangle:=\frac{1}{\sqrt{m_{1} ! m_{2} !}}\left(b_{1}^{\dagger}\right)^{m_{1}}\left(b_{2}^{\dagger}\right)^{m_{2}}|0\rangle
$$

in the infinite dimensional Hilbert space $\ell_{2}(N)$. Here $m_{1}, m_{2}=0,1,2, \ldots, N-1$ where we choose the order of the number pairs $\left(m_{1}, m_{2}\right)$ to be

$$
(0,0),(1,0),(0,1),(2,0),(1,1),(0,2),(3,0),(2,1),(1,2),(0,3), \ldots,(0, N-1) \text {. }
$$

$N$ is the number of the last energy shell to be considered. The dimension of the matrix is then given by

$$
M=\frac{N(N-1)}{2}
$$


and the orthogonality condition becomes

$$
\left\langle r_{2}, r_{1} \mid m_{1}, m_{2}\right\rangle=\delta_{r_{1}, m_{1}} \delta_{r_{2}, m_{2}} .
$$

The following recursion relations hold

$$
\begin{aligned}
b_{1}^{\dagger}\left|m_{1}, m_{2}\right\rangle & =\sqrt{m_{1}+1}\left|m_{1}+1, m_{2}\right\rangle ; & b_{2}^{\dagger}\left|m_{1}, m_{2}\right\rangle & =\sqrt{m_{2}+1}\left|m_{1}, m_{2}+1\right\rangle \\
b_{1}\left|m_{1}, m_{2}\right\rangle & =\sqrt{m_{1}}\left|m_{1}-1, m_{2}\right\rangle ; & & b_{2}\left|m_{1}, m_{2}\right\rangle=\sqrt{m_{2}}\left|m_{1}, m_{2}-1\right\rangle \\
\hat{n}_{1}\left|m_{1}, m_{2}\right\rangle & =m_{1}\left|m_{1}, m_{2}\right\rangle ; & & \hat{n}_{2}\left|m_{1}, m_{2}\right\rangle=m_{2}\left|m_{1}, m_{2}\right\rangle .
\end{aligned}
$$

With the relations in (A.19) and the orthogonality condition in (A.18) we obtain the following matrix representation for $\hat{H}$ where (with $\left.\left\langle r_{2}, r_{1}\left|\hat{H}_{0}\right| m_{1}, m_{2}\right\rangle=H_{k l}^{0}\right)$

$$
\begin{aligned}
H_{k l}^{0} & =\left[\frac{3}{4}+\frac{1}{4}\left(m_{1}^{2}+m_{2}^{2}\right)+\frac{3}{4}\left(m_{1}+m_{2}\right)\right] \delta_{r_{1}, m_{1}} \delta_{r_{2}, m_{2}} \\
& +\frac{m_{1}}{6} \sqrt{\left(m_{1}+1\right)\left(m_{1}+2\right)} \delta_{r_{1}, m_{1}+2} \delta_{r_{2}, m_{2}}+\frac{m_{2}}{6} \sqrt{\left(m_{2}+1\right)\left(m_{2}+2\right)} \delta_{r_{1}, m_{1}} \delta_{r_{2}, m_{2}+2} \\
& +\frac{m_{1}-2}{6} \sqrt{m_{1}\left(m_{1}-1\right)} \delta_{r_{1}, m_{1}-2} \delta_{r_{2}, m_{2}}+\frac{m_{2}-2}{6} \sqrt{m_{2}\left(m_{2}-1\right)} \delta_{r_{1}, m_{1}} \delta_{r_{2}, m_{2}-2} \\
& +\frac{1}{24} \sqrt{\left(m_{1}+1\right)\left(m_{1}+2\right)\left(m_{1}+3\right)\left(m_{1}+4\right)} \delta_{r_{1}, m_{1}+4} \delta_{r_{2}, m_{2}} \\
& +\frac{1}{24} \sqrt{\left(m_{2}+1\right)\left(m_{2}+2\right)\left(m_{2}+3\right)\left(m_{2}+4\right)} \delta_{r_{1}, m_{1}} \delta_{r_{2}, m_{2}+4} \\
& +\frac{1}{24} \sqrt{m_{1}\left(m_{1}-1\right)\left(m_{1}-2\right)\left(m_{1}-3\right)} \delta_{r_{1}, m_{1}-4} \delta_{r_{2}, m_{2}} \\
& +\frac{1}{24} \sqrt{m_{2}\left(m_{2}-1\right)\left(m_{2}-2\right)\left(m_{2}-3\right)} \delta_{r_{1}, m_{1}} \delta_{r_{2}, m_{2}-4}
\end{aligned}
$$

and (with $\left\langle r_{2}, r_{1}\left|\hat{H}_{1}\right| m_{1}, m_{2}\right\rangle=H_{k l}^{1}$ )

$$
\begin{aligned}
H_{k l}^{1} & =\frac{-1}{12}\left\{\left[\left(2 m_{1}+1\right)\left(2 m_{2}+1\right)\right] \delta_{r_{1}, m_{1}} \delta_{r_{2}, m_{2}}\right. \\
& +\left(2 m_{2}+1\right) \sqrt{\left(m_{1}+1\right)\left(m_{1}+2\right)} \delta_{r_{1}, m_{1}+2} \delta_{r_{2}, m_{2}} \\
& +\left(2 m_{1}+1\right) \sqrt{\left(m_{2}+1\right)\left(m_{2}+2\right)} \delta_{r_{1}, m_{1}} \delta_{r_{2}, m_{2}+2} \\
& +\left(2 m_{2}+1\right) \sqrt{m_{1}\left(m_{1}-1\right)} \delta_{r_{1}, m_{1}-2} \delta_{r_{2}, m_{2}} \\
& +\left(2 m_{1}+1\right) \sqrt{m_{2}\left(m_{2}-1\right)} \delta_{r_{1}, m_{1}} \delta_{r_{2}, m_{2}-2} \\
& +\sqrt{\left(m_{1}+1\right)\left(m_{1}+2\right)\left(m_{2}+1\right)\left(m_{2}+2\right)} \delta_{r_{1}, m_{1}+2} \delta_{r_{2}, m_{2}+2} \\
& +\sqrt{\left(m_{1}+1\right)\left(m_{1}+2\right)\left(m_{2}-1\right)\left(m_{2}-2\right)} \delta_{r_{1}, m_{1}+2} \delta_{r_{2}, m_{2}-2} \\
& +\sqrt{m_{1}\left(m_{1}-1\right)\left(m_{2}+1\right)\left(m_{2}+2\right)} \delta_{r_{1}, m_{1}-2} \delta_{r_{2}, m_{2}+2} \\
& \left.+\sqrt{m_{1} m_{2}\left(m_{1}-1\right)\left(m_{2}-1\right)} \delta_{r_{1}, m_{1}-2} \delta_{r_{2}, m_{2}-2}\right\}
\end{aligned}
$$

with $k, l=1,2 \ldots, M$. 


\begin{tabular}{||c|c|c|c|r|r||}
\hline & $E$ & $C_{2}$ & $2 C_{4}$ & $2 \sigma$ & $2 \sigma^{\prime}$ \\
\hline \hline$A_{1}$ & 1 & 1 & 1 & 1 & 1 \\
$A_{2}$ & 1 & 1 & 1 & -1 & -1 \\
$B_{1}$ & 1 & 1 & -1 & 1 & -1 \\
$B_{2}$ & 1 & 1 & -1 & -1 & 1 \\
$E_{1}$ & 2 & -2 & 0 & 0 & 0 \\
\hline
\end{tabular}

Table A.1: Character table for the $C_{4 v}$ symmetry group.

\section{A.2.2 Reduced Matrix Representation}

The matrix Hamiltonian $H=H_{k l}^{0}+\lambda H_{k l}^{1}$ has multiple degeneracies. For the purpose of our study it is essential to work with an irreducible representation of the Hamiltonian matrix so that no degeneracies, and only avoided crossings, occur (see Chapter 4). This is achieved by using group theoretical methods.

We note that the Hamiltonian $\tilde{H}$ in (A.9) is invariant under the following transformations

$$
\begin{aligned}
& \tilde{q}_{1} \quad \rightarrow \quad \tilde{q}_{1}, \quad \tilde{q}_{2} \quad \rightarrow \quad \tilde{q}_{2} \\
& \tilde{q}_{1} \quad \rightarrow \quad \tilde{q}_{2}, \quad \tilde{q}_{2} \rightarrow-\tilde{q}_{1} \\
& \tilde{q}_{1} \rightarrow-\tilde{q}_{1}, \quad \tilde{q}_{2} \rightarrow-\tilde{q}_{2} \\
& \tilde{q}_{1} \rightarrow-\tilde{q}_{2}, \quad \begin{array}{llll}
\tilde{q}_{2} & \rightarrow & \tilde{q}_{1}
\end{array} \\
& \tilde{q}_{1} \quad \rightarrow \quad-\tilde{q}_{1}, \quad \tilde{q}_{2} \quad \rightarrow \quad \tilde{q}_{2} \\
& \tilde{q}_{1} \quad \rightarrow \quad \tilde{q}_{1}, \quad \tilde{q}_{2} \quad \rightarrow-\tilde{q}_{2} \\
& \tilde{q}_{1} \quad \rightarrow \quad \tilde{q}_{2}, \quad \tilde{q}_{2} \quad \rightarrow \quad \tilde{q}_{1} \\
& \tilde{q}_{1} \rightarrow-\tilde{q}_{2}, \quad \tilde{q}_{2} \rightarrow-\tilde{q}_{1}
\end{aligned}
$$

and we say that $\tilde{H}$ admits the symmetries listed in (A.22). These transformations can be seen as rotations and reflections of a square as depicted in Fig. (A.1). The symmetries listed in (A.22) are the same as those for the $C_{4 v}$ point group; $H$ therefore has $C_{4 v}$ symmetry. This group has 8 elements:

$$
E, C_{2}, C_{4}, C_{4}^{3}, \sigma_{1}, \sigma_{2}, \sigma_{1}^{\prime}, \sigma_{2}^{\prime} \text {. }
$$

Here, $E$ is the unit element, $C_{2}, C_{4}$ and $C_{4}^{3}$ are rotations by $90^{\circ}, 180^{\circ}$ and $270^{\circ}$ respectively. $\sigma_{1}^{\prime}$ and $\sigma_{2}^{\prime}$ are reflections about the $\tilde{q}_{1}$ and $\tilde{q}_{2}$ axes whilst $\sigma_{1}$ and $\sigma_{2}$ are reflections about the same axes rotated by $45^{\circ}$ (refer to Fig. (A.1)). This group can be used to reduce the Hilbert space of $\tilde{H}$ into 5 irreducible subspaces. There are four one dimensional representations denoted by $A_{1}, A_{2}, B_{1}, B_{2}$ and one two dimensional representation $E_{1}[\mathrm{PE} 81]^{3}$. The character table is given in Table (A.1).

\footnotetext{
${ }^{3}$ Pullen and Edmonds studied the system with $H=\left(p_{1}^{2}+p_{2}^{2}+q_{1}^{2}+q_{2}^{2}\right) / 2+\alpha q_{1}^{2} q_{2}^{2}$ which also admits the $C_{4 v}$ symmetry group.
} 


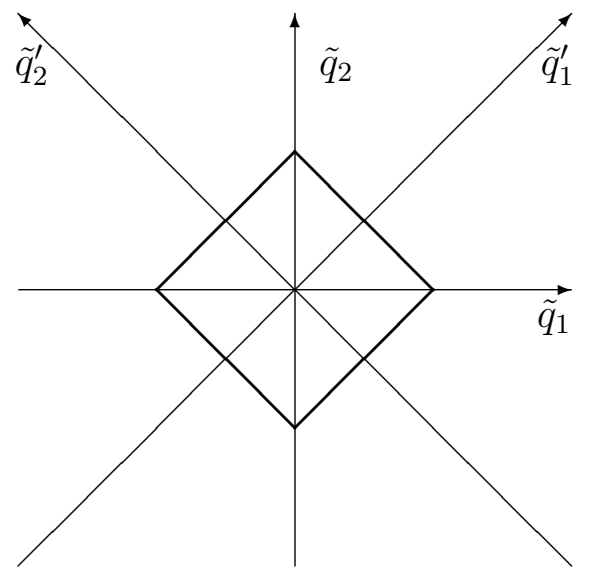

Figure A.1: The Hamiltonian $H$ is invariant under rotations and reflections of the square. $\tilde{q}_{1}^{\prime}$ and $\tilde{q}_{2}^{\prime}$ are the $\tilde{q}_{1}$ and $\tilde{q}_{2}$ axes rotated by $\pi / 4$.

The bases for the second quantised Hamiltonian $\hat{H}$ are obtained in the irreducible subspaces. They are calculated to be linear combinations of the eigenfunctions of the two dimensional unperturbed harmonic oscillator

$$
\begin{aligned}
A_{1} \rightarrow\left|m_{1}, m_{2}\right\rangle & =\left[2+2 \delta_{m_{1}, m_{2}}\right]^{1 / 2}\left(\left|2 m_{1}, 2 m_{2}\right\rangle+\left|2 m_{2}, 2 m_{1}\right\rangle\right) \\
A_{2} \rightarrow\left|m_{1}, m_{2}\right\rangle & =\left[2-2 \delta_{2 m_{1}-1,2 m_{2}-1}\right]^{1 / 2}\left(\left|2 m_{1}-1,2 m_{2}-1\right\rangle-\left|2 m_{2}-1,2 m_{1}-1\right\rangle\right] \\
B_{1} \rightarrow\left|m_{1}, m_{2}\right\rangle & =\left[2-2 \delta_{m_{1}, m_{2}}\right]^{1 / 2}\left(\left|2 m_{1}, 2 m_{2}\right\rangle-\left|2 m_{2}, 2 m_{1}\right\rangle\right) \\
B_{2} \rightarrow\left|m_{1}, m_{2}\right\rangle & =\left[2+2 \delta_{2 m_{1}-1,2 m_{2}-1}\right]^{1 / 2}\left(\left|2 m_{1}-1,2 m_{2}-1\right\rangle+\left|2 m_{2}-1,2 m_{1}-1\right\rangle\right) \\
E_{1} \rightarrow\left|m_{1}, m_{2}\right\rangle & =\left[2+2 \delta_{m_{1}, m_{2}} \delta_{2 m_{1}-1,2 m_{2}-1}\right]^{1 / 2}\left(\left|2 m_{1}, 2 m_{2}-1\right\rangle+\left|2 m_{2}-1,2 m_{1}\right\rangle\right) .
\end{aligned}
$$

We chose the subspace of positive parity ${ }^{4}$ to do our calculations in i.e., the $A_{1}$ subspace with the basis given by (A.23). We note that, by using this basis, the number pairs $(r, s)$ and $(s, r)$ will lead to the same state. To avoid this, the order of the number pairs are taken to be

$$
\left(m_{1}, m_{2}\right)=(0,0),(1,0),(2,0),(1,1),(3,0),(2,1),(4,0),(3,1),(2,2),(5,0) \ldots
$$

Using the same notation as before we calculate the matrix representations of $\hat{H}_{0}$ and $\hat{H}_{1}$ in the $A_{1}$ subspace viz.

$$
\begin{aligned}
H_{k l}^{0} & =\frac{1}{24}\left[\left(1+\delta_{m_{1}, m_{2}}\right)\left(1+\delta_{r_{1}, r_{2}}\right)\right]^{1 / 2} \times \\
& \times\left\{\left[24\left(m_{1}^{2}+m_{2}^{2}\right)+36\left(m_{1}+m_{2}\right)+18\right]\left[\delta_{r_{1}, m_{1}} \delta_{r_{2}, m_{2}}+\delta_{r_{1}, m_{2}} \delta_{r_{2}, m_{1}}\right]+\right.
\end{aligned}
$$

\footnotetext{
${ }^{4}$ The states are symmetric under permutations of $\tilde{q}_{1}$ and $\tilde{q}_{2}$.
} 


$$
\begin{aligned}
& +8 m_{1} \sqrt{\left(2 m_{1}+1\right)\left(2 m_{1}+2\right)}\left[\delta_{r_{1}, m_{1}+1} \delta_{r_{2}, m_{2}}+\delta_{r_{1}, m_{2}} \delta_{r_{2}, m_{1}+1}\right] \\
& +8 m_{2} \sqrt{\left(2 m_{2}+1\right)\left(2 m_{2}+2\right)}\left[\delta_{r_{1}, m_{1}} \delta_{r_{2}, m_{2}+1}+\delta_{r_{1}, m_{2}+1} \delta_{r_{2}, m_{1}}\right] \\
& +8\left(m_{1}-1\right) \sqrt{2 m_{1}\left(2 m_{1}-1\right)}\left[\delta_{r_{1}, m_{1}-1} \delta_{r_{2}, m_{2}}+\delta_{r_{1}, m_{2}} \delta_{r_{2}, m_{1}-1}\right] \\
& +8\left(m_{2}-1\right) \sqrt{2 m_{2}\left(2 m_{2}-1\right)}\left[\delta_{r_{1}, m_{1}} \delta_{r_{2}, m_{2}-1}+\delta_{r_{1}, m_{2}-1} \delta_{r_{2}, m_{1}}\right] \\
& +\sqrt{\left(2 m_{1}+1\right)\left(2 m_{1}+2\right)\left(2 m_{1}+3\right)\left(2 m_{1}+4\right)}\left[\delta_{r_{1}, m_{1}+2} \delta_{r_{2}, m_{2}}+\delta_{r_{1}, m_{2}} \delta_{r_{2}, m_{1}+2}\right] \\
& +\sqrt{\left(2 m_{2}+1\right)\left(2 m_{2}+2\right)\left(2 m_{2}+3\right)\left(2 m_{2}+4\right)}\left[\delta_{r_{1}, m_{1}} \delta_{r_{2}, m_{2}+2}+\delta_{r_{1}, m_{2}+2} \delta_{r_{2}, m_{1}}\right] \\
& +\sqrt{2 m_{1}\left(2 m_{1}-1\right)\left(2 m_{1}-2\right)\left(2 m_{1}-3\right)}\left[\delta_{r_{1}, m_{1}-2} \delta_{r_{2}, m_{2}}+\delta_{r_{1}, m_{2}} \delta_{r_{2}, m_{1}-2}\right] \\
& \left.\left.+\sqrt{2 m_{2}\left(2 m_{2}-1\right)\left(2 m_{2}-2\right)\left(2 m_{2}-3\right)}\left[\delta_{r_{1}, m_{1}} \delta_{r_{2}, m_{2}-2}+\delta_{r_{1}, m_{2}-2} \delta_{r_{2}, m_{1}}\right]\right\} \text { A. } 25\right)
\end{aligned}
$$

and

$$
\begin{aligned}
H_{k l}^{1} & =\frac{-1}{12}\left[\left(1+\delta_{m_{1}, m}\right)\left(1+\delta_{r_{1}, r_{2}}\right)\right]^{1 / 2} \times \\
& \times\left\{\left[4\left(m_{1}+m_{2}\right)+16 m_{1} m_{2}+1\right]\left[\delta_{r_{1}, m_{1}} \delta_{r_{2}, m_{2}}+\delta_{r_{1}, m_{2}} \delta_{r_{2}, m_{1}}\right]\right. \\
& +\left(1+4 m_{2}\right) \sqrt{\left(2 m_{1}+1\right)\left(2 m_{1}+2\right)}\left[\delta_{r_{1}, m_{1}+1} \delta_{r_{2}, m_{2}}+\delta_{r_{1}, m_{2}} \delta_{r_{2}, m_{1}+1}\right] \\
& +\left(1+4 m_{1}\right) \sqrt{\left(2 m_{2}+1\right)\left(2 m_{2}+2\right)}\left[\delta_{r_{1}, m_{1}} \delta_{r_{2}, m_{2}+1}+\delta_{r_{1}, m_{2}+1} \delta_{r_{2}, m_{1}}\right] \\
& +\left(1+4 m_{2}\right) \sqrt{2 m_{1}\left(2 m_{1}-1\right)}\left[\delta_{r_{1}, m_{1}-1} \delta_{r_{2}, m_{2}}+\delta_{r_{1}, m_{2}} \delta_{r_{2}, m_{1}-1}\right] \\
& +\left(1+4 m_{1}\right) \sqrt{2 m_{2}\left(2 m_{2}-1\right)}\left[\delta_{r_{1}, m_{1}} \delta_{r_{2}, m_{2}-1}+\delta_{r_{1}, m_{2}-1} \delta_{r_{2}, m_{1}}\right] \\
& +\sqrt{\left(2 m_{1}+1\right)\left(2 m_{1}+2\right)\left(2 m_{2}+1\right)\left(2 m_{2}+2\right)}\left[\delta_{r_{1}, m_{1}+1} \delta_{r_{2}, m_{2}+1}+\delta_{r_{1}, m_{2}+1} \delta_{r_{2}, m_{1}+1}\right] \\
& +\sqrt{\left(2 m_{1}+1\right)\left(2 m_{1}+2\right) 2 m_{2}\left(2 m_{2}-1\right)}\left[\delta_{r_{1}, m_{1}+1} \delta_{r_{2}, m_{2}-1}+\delta_{r_{1}, m_{2}-1} \delta_{r_{2}, m_{1}+1}\right] \\
& +\sqrt{2 m_{1}\left(2 m_{1}-1\right)\left(2 m_{2}+1\right)\left(2 m_{2}+2\right)}\left[\delta_{r_{1}, m_{1}-1} \delta_{r_{2}, m_{2}+1}+\delta_{r_{1}, m_{2}+1} \delta_{r_{2}, m_{1}-1}\right] \\
& \left.\left.+2 \sqrt{m_{1} m_{2}\left(2 m_{1}-1\right)\left(2 m_{2}-1\right)}\left[\delta_{r_{1}, m_{1}-1} \delta_{r_{2}, m_{2}-1}+\delta_{r_{1}, m_{2}-1} \delta_{r_{2}, m_{1}-1}\right]\right\} . \quad \text { A. } 26\right)
\end{aligned}
$$

Here $H_{k l}$ is a strictly banded matrix. 
158 APPENDIX A. QUARTIC OSCILLATOR: MATRIX REPRESENTATION 


\section{Appendix B}

\section{Position Operator: Matrix Representation}

Let

$$
H(\mathbf{p}, \mathbf{q})=H_{0}(\mathbf{p}, \mathbf{q})+\lambda H_{1}(\mathbf{q}) .
$$

The matrix representation of $H_{1}$ can be obtained, in the basis of diagonal $H_{0}$, without explicit knowledge of this basis. This Appendix describes an easy to implement numerical procedure to calculate the matrix representation of the position operator, if only the eigenvalues of the underlying Hamiltonian are known [KH 92a] which can then be used to calculate $H_{1}$.

\section{B.1 Matrix Representation of Position Operator}

Let the discrete, nondegenerate spectrum of the Hamilton operator $\hat{H}_{0}$ be known, i.e.

$$
\left\langle m\left|\hat{H}_{0}\right| n\right\rangle=E_{n} \delta_{m n} ; \quad n, m=0,1,2, \ldots, N .
$$

If $\hat{H}_{0}$ contains kinetic energy terms and a local potential $V$ viz.

$$
\hat{H}_{0}=-\frac{1}{2} \sum_{j=1}^{P} \frac{\partial^{2}}{\partial q_{j}^{2}}+V\left(\hat{q}_{1}, \hat{q}_{2}, \ldots, \hat{q}_{P}\right)
$$

with $P$ the dimension of the system. The following commutator holds

$$
\left[\hat{q}_{k}, \hat{H}_{0}\right]=i \hat{p}_{k} ; \quad k=1,2, \ldots, P .
$$

Now let

$$
E_{n} \delta_{n m}=D_{n m}
$$

where $D_{n m}$ is the diagonal matrix with the eigenvalues of $H_{0}$ on the diagonal. We exploit the commutator $\left[\hat{q}_{r}, \hat{p}_{s}\right]=i \delta_{r s}$ and the completeness relation. 
We aim at calculating $\left\langle m\left|\hat{q}_{i}\right| n\right\rangle$ without explicitly using the state vectors $|n\rangle$ in (B.1). This is accomplished by making use of the matrix representation of the commutator

$$
\left\langle m\left|\left[\hat{q}_{r}, \hat{p}_{s}\right]\right| n\right\rangle=\sum_{k}\left[\left\langle m\left|\hat{q}_{r}\right| k\right\rangle\left\langle k\left|\hat{p}_{s}\right| n\right\rangle-\left\langle m\left|\hat{p}_{s}\right| k\right\rangle\left\langle k\left|\hat{q}_{r}\right| n\right\rangle\right]=i \delta_{m n} \delta_{r s} .
$$

From (B.2) and using (B.1) one obtains

$$
\left\langle m\left|\hat{p}_{s}\right| n\right\rangle=-i \sum_{k}\left(\left\langle m\left|\hat{q}_{s}\right| k\right\rangle D_{k n}-D_{m k}\left\langle k\left|\hat{q}_{s}\right| n\right\rangle\right)
$$

and then substituting (B.5) back into (B.4) leads to

$$
\begin{aligned}
\delta_{m n} \delta_{r s}= & \sum_{k k^{\prime}}\left[2\left\langle m\left|\hat{q}_{r}\right| k\right\rangle D_{k k^{\prime}}\left\langle k^{\prime}\left|\hat{q}_{s}\right| n\right\rangle-\left\langle m\left|\hat{q}_{r}\right| k\right\rangle\left\langle k\left|\hat{q}_{s}\right| k^{\prime}\right\rangle D_{k^{\prime} n}\right. \\
& \left.-D_{m k^{\prime}}\left\langle k^{\prime}\left|\hat{q}_{s}\right| k\right\rangle\left\langle k\left|\hat{q}_{r}\right| n\right\rangle\right] .
\end{aligned}
$$

If we put $\left\langle m\left|\hat{q}_{r}\right| n\right\rangle:=Q_{n m}^{(r)}$ we have

$$
-Q_{m k}^{(r)} Q_{k k^{\prime}}^{(s)} D_{k^{\prime} n}-D_{m k^{\prime}} Q_{k^{\prime} k}^{(s)} Q_{k n}^{(r)}+2 Q_{m k}^{(r)} D_{k k^{\prime}} Q_{k^{\prime} n}^{(s)}=\delta_{n m} \delta_{r s}
$$

where we sum over double indices.

As it stands the relation in (B.7) holds in an infinite dimensional space. We also recall that the operators $\hat{q}$ and $\hat{p}$ are unbounded. The matrices $Q$ and $D$ will thus also be infinite dimensional matrices representing unbounded operators. To be able to handle them numerically, $Q$ and $D$ have to be truncated. Then, however, (B.7) can not hold because for finite dimensions the trace of a commutator vanishes as does the trace of the left hand side of (B.7); however, the trace (over labels $n$ and $m$ ) of the right hand side is equal to $N$ where $N$ is the dimension of the truncated matrices $Q$ and $D$ ( $N$ is conveniently chosen even). To get as close as possible to (B.7) we replace $\delta_{n m}$ by a modified matrix $C$ where

$$
C=\left(\begin{array}{cccccc}
1 & 0 & 0 & \cdots & 0 & * \\
0 & 1 & 0 & \cdots & 0 & * \\
0 & 0 & 1 & \cdots & 0 & * \\
\vdots & \vdots & \vdots & \ddots & \vdots & \vdots \\
0 & 0 & 0 & \cdots & 1 & * \\
* & * & * & \cdots & * & -(N-1)
\end{array}\right)
$$

With $\operatorname{tr}(C)=0$, the following modified equation is considered for $N$ finite

$$
-Q_{m k}^{(r)} Q_{k k^{\prime}}^{(s)} D_{k^{\prime} n}-D_{m k^{\prime}} Q_{k^{\prime} k}^{(s)} Q_{k n}^{(r)}+2 Q_{m k}^{(r)} D_{k k^{\prime}} Q_{k^{\prime} n}^{(s)}=C .
$$

In (B.8) the asterisks are elements that might be nonzero, but their values are of no importance for our procedure. Equation (B.9) is a set of 6 nonlinear equations (in 
the superscripts $k$ and $n$ for three dimensions) and can be solved for $Q_{n m}$. Further on we only consider the one dimensional case and drop the superscripts. The matrix $C$ has also been chosen in this particular way to get as close as possible to the actual infinite dimensional problem in the top left corner of the truncated problem. For the one dimensional harmonic oscillator we have tested this problem and the known entries of the infinite dimensional matrix coincide exactly with the top left corner entries of the truncated problem.

We demonstrate the explicit procedure for the simpler case of good parity as this cuts down the number of equations by a factor two. In this case, the symmetric matrix $Q_{n m}$ has zeros on the diagonal and consists of two disjoint matrices $Q_{n m}^{+}$(even parity) and $Q_{n m}^{-}$(odd parity) so that

$$
Q_{n m}=Q_{n m}^{+} \oplus Q_{n m}^{-}
$$

This reduces the problem so that only the even parity part, which has the following form,

$$
Q_{n m}^{+}=\left(\begin{array}{cccccc}
0 & * & 0 & * & 0 & \ldots \\
* & 0 & * & 0 & * & \ldots \\
0 & * & 0 & * & 0 & \ldots \\
\vdots & \vdots & \vdots & \vdots & \ddots & \vdots
\end{array}\right)
$$

need be considered. The asterisks denote the nonzero elements of the matrix. $Q_{n m}^{ \pm}$ has $N^{2} / 4$ independent variables so that (B.9) will lead to a system of $N^{2} / 4$ nonlinear equations in $N^{2} / 4$ unknowns.

In implementing (B.9) (for even parity) the following system of equations is obtained

$$
\begin{aligned}
& \sum_{m} Q_{i m} Q_{m j}\left(2 \epsilon_{m}-\epsilon_{i}-\epsilon_{j}\right)=\delta_{i j} \\
& \sum_{n} Q_{k n} Q_{n l}\left(2 \epsilon_{n}-\epsilon_{k}-\epsilon_{l}\right)=\delta_{k l}
\end{aligned}
$$

where (both equations have to be considered simultaneously)

$$
\begin{aligned}
i & =1,3,5, \ldots, N-1 \\
j & =i,(i+2),(i+4), \ldots,(N-1) \\
m & =2,4, \ldots, N \\
k & =2,4,6, \ldots, N-2 \\
l & =i,(k+2),(k+4), \ldots,(N-2) \\
n & =1,3,5, \ldots, N-1
\end{aligned}
$$

and $\epsilon_{i}$ is the $i$-th eigenvalue of $H_{0}$. The quadratic equation in (B.9) can be solved by solving the set of nonlinear equations in (B.10) and using for instance the NewtonRaphson method. The Jacobian can be obtained straightforwardly. If a guess-value is needed a good choice might be $Q_{n m}$ for the harmonic oscillator which is explicitly known [KH 92a]. 


\section{B.2 Representation of Quartic Oscillator}

To obtain $Q_{n m}$ from the spectrum of $\hat{H}_{0}$ alone is of interest for its own sake. In addition, we have tested and in fact successfully taken advantage of the method by calculating the spectrum for a Hamilton operator $\hat{H}$ where (with $H_{1}$ local)

$$
\hat{H}(\mathbf{p}, \mathbf{q})=\hat{H}_{0}(\mathbf{p}, \mathbf{q})+\lambda \hat{H}_{1}(\mathbf{q}) .
$$

For our study we considered [HK 91]

$$
\begin{gathered}
\hat{H}_{0}=\frac{1}{2}\left[\hat{p}_{1}^{2}+\hat{p}_{2}^{2}\right]+\frac{1}{6}\left[\hat{q}_{1}^{4}+\hat{q}_{2}^{4}\right] \\
\hat{H}_{1}=-\frac{1}{3} \hat{q}_{1}^{2} \hat{q}_{2}^{2} .
\end{gathered}
$$

For the specific type of our investigation we required a representation in which $\hat{H}_{0}$ is diagonal - the corresponding spectrum is obtained by standard procedure. To get the representation of $\hat{H}_{1}$ in that basis our procedure turned out to be very reliable and stable for dimensions up to 100 in comparison with the method used in the references. The limit of 100 is imposed because of the computing power at hand. It can be increased if more computing power is available [KH 92a].

Because of the wide variety of Hamiltonians that can be decomposed in a similar way as $\hat{H}$ in (B.11), this method is rather general and can help to determine the eigenvalues and eigenvectors of the full Hamiltonian. With supercomputers, the limit on the number of equations that can be solved simultaneously can be rather large, in which case this method proves its full potential. 


\section{Appendix C}

\section{Unfolding: Semiclassical Method}

In general, the Hamiltonians we study have the form

$$
H(\mathbf{p}, \mathbf{q})=\sum_{i=1}^{f} \frac{p_{i}^{2}}{2}+V(\mathbf{q})
$$

with $f$ the number of degrees of freedom of the system and $\mathbf{q}=\left(q_{1}, q_{2}, \ldots, q_{f}\right)$. For $\tilde{H}$ in (A.9) $f=2$. To unfold the spectrum of such a Hamiltonian we need to calculate $N_{a v}(E)$ (see $\S 3.4$ ). Asymptotically, $N_{a v}(E)$ can be obtained by a semiclassical fit of the staircase function to obtain Weyl's rule [Oz 88]

$$
N_{a v}(E) \approx \frac{V(E)}{(2 \pi \hbar)^{f}}
$$

$V(E)$ is the volume of the classical phase space of dimension $N$ and is calculated through the following integral

$$
V(E)=\int_{\mathcal{R}^{f}} \int_{\mathcal{R}^{f}} d^{f} p d^{f} q \Theta[E-H(\vec{p}, \vec{q})]
$$

with $\Theta$ the unit step function.

For the quartic oscillator we make the following ansatz

$$
\mathbf{p}=E^{\frac{1}{2}} \mathbf{p}^{\prime} ; \quad \mathbf{q}=E^{\frac{1}{n}} \mathbf{q}^{\prime}
$$

with $n$ the rank of $V$. We consequently obtain

$$
\begin{aligned}
& H(\mathbf{p}, \mathbf{q})=E H\left(\mathbf{p}^{\prime}, \mathbf{q}^{\prime}\right) \\
& d^{f} p d^{f} q=E^{\frac{(n+2)}{2 n} f} d^{f} p^{\prime} d^{f} q^{\prime}
\end{aligned}
$$

so that

$$
V(E)=E^{\frac{(n+2)}{2 n} f} \iint \Theta\left[E\left(1-H\left(\mathbf{p}^{\prime}, \mathbf{q}^{\prime}\right)\right] d^{f} q^{\prime} d^{f} q^{\prime}\right.
$$


The integral is constant for $E \rightarrow \infty$ and

$$
N_{a v}(E) \approx V(E)=C E^{\frac{(n+2)}{2 n} f} .
$$

For the quartic oscillator $C=1, f=2$ and $n=4$ so that

$$
N_{a v}(E) \approx E^{\frac{3}{2}} .
$$




\section{Appendix D}

\section{The Hydrogen Atom in a Magnetic Field}

\section{D.1 The Hamiltonian}

The magnetic force on an electric charge $e$ has its origin in the theory of relativity. It arises only when the charge is moving and its strength is proportional to the ratio of its velocity to the velocity of light.

The Hydrogen atom is a two particle system, one proton and one electron. However, after a reduction to a reduced mass system, it can be considered as a one particle system where a particle of mass $m$ and negative charge $e$ is moving in a magnetic field $\mathbf{B}$. The Hamiltonian is given by

$$
H=\frac{1}{2 m}\left(\mathbf{p}+\frac{e}{c} \mathbf{A}\right)^{2}+e \phi
$$

with $\mathbf{p}=-i \hbar \nabla, c$ the velocity of light, $\mathbf{A}$ the vector potential of $\mathbf{B}$ and $\phi$ a scalar potential denoting the Coulomb interaction between the proton and electron. The vector potential satisfies $\nabla \times \mathbf{A}=\mathbf{B}$ and $\nabla \cdot \mathbf{B}=0$.

Now, let $\mathbf{B}$ have a uniform strength directed along the $z$-axis i.e. $\mathbf{B}=B \hat{z}$. Then

$$
\mathbf{A}=\frac{1}{2} \mathbf{B} \times \mathbf{r}
$$

called the symmetry gauge, which satisfies $\nabla \cdot \mathbf{A}=0$. Denoting the classical orbital angular momentum of a charged particle as $\mathbf{L}=\mathbf{r} \times \mathbf{p}$, the Hamiltonian can be written, in Cartesian coordinates,

$$
H=\frac{p^{2}}{2 m}-\frac{e^{2}}{r}+\frac{e}{2 m c} B l_{z}+\frac{e^{2} B^{2}}{8 m c^{2}}\left(x^{2}+y^{2}\right)
$$

with $l_{z}$ the $z$-component of the angular momentum. The right-hand side contains the kinetic energy, the Coulomb potential, the (paramagnetic) Zeeman energy and the (diamagnetic) quadratic term. 
Let the angular frequency $\omega$ (of the classical particle), be half the cyclotron frequency where

so that, at a field strength of

$$
\omega=\frac{1}{2} \omega_{c}=\frac{e B}{2 m c}
$$

$$
B=B_{0}=\frac{m^{2} e^{3} c}{\hbar^{3}} \simeq 2.35 \times 10^{5} \mathrm{~T},
$$

the oscillator energy $\hbar \omega$ equals the Rydberg energy where

$$
\operatorname{Ryd}=\frac{m e^{4}}{2 \hbar^{2}} \simeq 13.6 \mathrm{eV}
$$

which is from now on the unit of measure of the energy.

In terms of a dimensionless field strength parameter

$$
\gamma=\frac{B}{B_{0}}=\frac{\hbar \omega}{\mathcal{R}}
$$

and using from now on atomic units $(\hbar=c=m=e=1)$ and spherical coordinates the Hamiltonian in (D.2) can be written

$$
H=\frac{p^{2}}{2}-\frac{1}{r}+\frac{\gamma}{2} l_{z}+\frac{\gamma^{2}}{8} r^{2} \sin ^{2} \theta .
$$

In this representation relativistic corrections to the simple model are negligible for fields with $\gamma<10^{4}$. In (D.5) the effects of spin-orbit coupling has been neglected which is permissible for strong fields where $\gamma n^{3}>10^{-4}$ with $n$ the principle quantum number [Ga 77].

In spherical coordinates the eigenfunctions for the unperturbed Hydrogen atom are

with

$$
|n, l, m\rangle=R_{n, l} Y_{l, m}
$$

$$
R_{n, l}(r)=\sqrt{\frac{(n-l-1) !}{(n+l) !}} \frac{2}{n^{2}}\left(\frac{2 r}{n}\right)^{l} e^{-r / n} L_{n-l-1}^{2 l+1}\left(\frac{2 r}{n}\right)
$$

and

$$
Y_{l, m}(\theta, \phi)=(-1)^{m} \sqrt{\frac{(2 l+1)(l-m) !}{2(l+m) !}} P_{l}^{m}(\cos \theta) e^{i m \phi}
$$

with $Y_{l, m}$ known as the spherical harmonics. Here $n$ is the principle, $l$ the angular momentum and $m$ the magnetic quantum numbers such that

$$
\begin{aligned}
n & =1,2,3, \ldots \\
l & =0,1,2, \ldots, n-1 \\
m & =-l,-l+1, \ldots, l-1, l .
\end{aligned}
$$

$L_{n-l-1}^{2 l+1}(2 r / n)$ is the generalised Laguerre polynomials and $P_{l}^{m}(\cos \theta)$ the generalised Legendre polynomials. 


\section{D.2 Schrödinger's Radial Integral}

\section{D.2.1 First Method}

We want to calculate integrals of the form

$$
J=\int_{0}^{\infty} x^{p} e^{-(\alpha+\beta) x / 2} L_{k-n}^{n}(\alpha x) L_{k^{\prime}-n^{\prime}}^{n^{\prime}}(\beta x) d x
$$

where the associated Laguerre polynomials $L_{q-p}^{p}$ are defined such that

$$
L_{p}^{k}=(-1)^{k} \frac{d^{k}}{d x^{k}} L_{p+k} ; \quad \text { with order } p .
$$

First, let $\alpha=\beta=1$ in (D.9) so that

$$
Q(x)=\int_{0}^{\infty} x^{n} e^{-x} L_{q-p}^{p}(x) L_{q^{\prime}-p^{\prime}}^{p^{\prime}}(x) d x
$$

where $n, q, p, q^{\prime}$ and $p^{\prime}$ are all integers. Use is made of the generating functions

$$
\begin{aligned}
\sum_{k=0}^{\infty} L_{q-p}^{p}(x) t^{k} & =(1-t)^{-p-1} \exp \left(\frac{t x}{t-1}\right) \\
\sum_{k^{\prime}=0}^{\infty} L_{q^{\prime}-p^{\prime}}^{p^{\prime}}(x) s^{k^{\prime}} & =(1-s)^{-p^{\prime}-1} \exp \left(\frac{s x}{s-1}\right)
\end{aligned}
$$

and we can say

$$
\sum_{k, k^{\prime}} L_{q-p}^{p} L_{q^{\prime}-p^{\prime}}^{p^{\prime}} t^{k} s^{k^{\prime}}=(1-t)^{-p-1}(1-s)^{-p^{\prime}-1} \exp \left(\left[\frac{t}{t-1}+\frac{s}{s-1}\right] x\right) .
$$

Now, multiply both sides with $e^{-x} x^{n}$ and integrate over the positive real axis

$$
\begin{aligned}
& \sum_{k, k^{\prime}} t^{k} s^{k^{\prime}} \int_{0}^{\infty} x^{n} e^{-x} L_{q-p}^{p}(x) L_{q^{\prime}-p^{\prime}}^{p^{\prime}}(x) d x \\
= & (1-t)^{-p-1}(1-s)^{-p^{\prime}-1} \int_{0}^{\infty} x^{n} \exp \left(\left[\frac{t}{t-1}+\frac{s}{s-1}-1\right] x\right) d x,
\end{aligned}
$$

make use of the integral

$$
\int_{0}^{\infty} x^{n} e^{-k x} d x=\frac{n !}{k^{n+1}}
$$

to obtain

$$
\int_{0}^{\infty} x^{n} \exp \left(-\left[\frac{t}{t-1}+\frac{s}{s-1}-1\right] x\right) d x=n !(1-t)^{n+1}(1-s)^{n+1}(1-s t)^{-n-1}
$$


so that

$$
\sum_{k, k^{\prime}} t^{k} s^{k^{\prime}} \int_{0}^{\infty} x^{n} e^{-x} L_{q-p}^{p}(x) L_{q^{\prime}-p^{\prime}}^{p^{\prime}}(x) d x=r !(1-t)^{n-p}(1-s)^{n-p^{\prime}}(1-s t)^{-n-1} .
$$

$Q$ is finally obtained by expanding the right-hand-side of (D.13) in powers of $t$ and $s$ and by seeking terms of the form $t^{k} s^{k^{\prime}}$ so that

$$
Q=n ! \sum_{\tau=0}^{\substack{q-p \\
q^{\prime}-p^{\prime}}}(-1)^{q+q^{\prime}-p-p^{\prime}+\tau}\left(\begin{array}{c}
n-p \\
q-p-\tau
\end{array}\right)\left(\begin{array}{c}
n-p^{\prime} \\
q^{\prime}-p^{\prime}-\tau
\end{array}\right)\left(\begin{array}{c}
-(n+1) \\
\tau
\end{array}\right)
$$

where $(n-p) \geq 0$ and $\left(n-p^{\prime}\right) \geq 0$. The binomial coefficients are defined such that

$$
\left(\begin{array}{l}
n \\
k
\end{array}\right)= \begin{cases}\frac{n !}{(n-k) ! k !} & n \geq 0 \\
(-)^{k} \frac{(-n+k-1) !}{(-n-1) ! k !} & n<0 \\
\text { undefined } & k<0\end{cases}
$$

To calculate the general integral in (D.9) let

$$
\frac{\alpha+\beta}{2} x=y
$$

Equation (D.16) can now be seen as the sum of the following two equations

$$
\begin{aligned}
\alpha x & =(1+\gamma) y \\
\beta x & =(1-\gamma) y \\
\text { with } \gamma & =\frac{\alpha-\beta}{\alpha+\beta} .
\end{aligned}
$$

With a change of coordinates to $y$

$$
J=\left(\frac{2}{\alpha+\beta}\right)^{p+1} \int y^{p} e^{-y} L_{n-k}^{n}((1+\gamma) y) L_{n^{\prime}-k^{\prime}}^{n^{\prime}}((1-\gamma) y) d y
$$

and by using the known relation [AS 65]

$$
L_{s}^{r}(\mu x)=\sum_{\lambda=0}^{s}\left(\begin{array}{c}
s+r \\
\lambda
\end{array}\right) \mu^{s-\lambda}(1-\mu)^{\lambda} L_{s-\lambda}^{r}(x)
$$

one obtains

$$
\begin{aligned}
J & =\left(\frac{2}{\alpha+\beta}\right)^{p+1} \sum_{\lambda=0}^{k-n} \sum_{\mu=0}^{k^{\prime}-n^{\prime}}(-)^{\lambda}\left(\begin{array}{c}
k \\
\lambda
\end{array}\right)\left(\begin{array}{c}
k^{\prime} \\
\mu
\end{array}\right) \gamma^{\lambda+\mu}(1+\gamma)^{k-n-\lambda}(1-\gamma)^{k^{\prime}-n^{\prime}-\mu} \\
& *\left\{\int_{0}^{\infty} y^{p} e^{-y} L_{k-n-\lambda}^{n}(y) L_{k^{\prime}-n^{\prime}-\mu}^{n^{\prime}}(y) d y\right\} .
\end{aligned}
$$


With the use of (D.14)

$$
\begin{aligned}
J & =\left(\frac{2}{\alpha+\beta}\right)^{p+1} p ! \sum_{\lambda=0}^{k-n} \sum_{\mu=0}^{k^{\prime}-n^{\prime}}(-)^{\lambda}\left(\begin{array}{c}
k \\
\lambda
\end{array}\right)\left(\begin{array}{c}
k^{\prime} \\
\mu
\end{array}\right) \gamma^{\lambda+\mu}(1+\gamma)^{k-n-\lambda}(1-\gamma)^{k^{\prime}-n^{\prime}-\mu} \\
& \left.*\left\{\begin{array}{c}
p-n \\
\sum_{\tau=0}^{k-n-\lambda} \\
k^{\prime}-n^{\prime}-\mu \\
k-n-\lambda-\tau
\end{array}\right)\left(\begin{array}{c}
p-n^{\prime} \\
k^{\prime}-n^{\prime}-\mu-\tau
\end{array}\right)\left(\begin{array}{c}
-p-1 \\
\tau
\end{array}\right)\right\}(\text { D.20) }
\end{aligned}
$$

with $C=k+k^{\prime}-\lambda-\mu+\tau$. Equation (D.20) is known as Schrödinger's radial integral. If $\alpha=\beta$ the integral is much easier to handle because $\gamma=0$ and the integral is then only defined if $\lambda=\mu=0$ and

$$
J^{(\alpha=\beta)}=\left(\frac{1}{\alpha}\right)^{p+1} p !\left\{\sum_{\tau=0}^{\leq \substack{k-n \\
k^{\prime}-n^{\prime}}}(-1)^{k+k^{\prime}+\tau}\left(\begin{array}{c}
p-n \\
k-n-\tau
\end{array}\right)\left(\begin{array}{c}
p-n^{\prime} \\
k^{\prime}-n^{\prime}-\tau
\end{array}\right)\left(\begin{array}{c}
-p-1 \\
\tau
\end{array}\right)\right\} .
$$

\section{D.2.2 Second Method}

Schrödinger attempted integrals of the form [Sc 26]

$$
J(x)=\int_{0}^{\infty} x^{p} e^{-(\alpha+\beta) x / 2} L_{n+k}^{n}(\alpha x) L_{n^{\prime}+k^{\prime}}^{n^{\prime}}(\beta x) d x
$$

with $L_{n+k}^{n}$ the associated Laguerre polynomials defined viz.

$$
L_{p}^{k}=\frac{d^{k}}{d x^{k}} L_{p} ; \quad \text { with order } k-p
$$

The same method as the previous paragraph can be employed where

$$
L_{n}^{m}(x)=(-)^{n} \frac{n !}{(n-m) !}\left[\sum_{k=0}^{n-m} \frac{(-)^{k}}{k !} \frac{n !(n-m) !}{(n-k) !(n-m-k) !} x^{n-m-k}\right]
$$

and after the transformation in (D.17)

$$
\begin{aligned}
L_{n+k}^{n}(\alpha x) & =\sum_{\lambda=0}^{k} \frac{\gamma^{\lambda} y^{\lambda}}{\lambda !} L_{n+k}^{n+\lambda}(y) \\
L_{n+k}^{n}(\beta x) & =\sum_{\mu=0}^{k}(-)^{\mu} \frac{\gamma^{\mu} y^{\mu}}{\mu !} L_{n+k}^{n+\mu}(y) \\
\gamma & =\frac{\alpha-\beta}{\alpha+\beta} .
\end{aligned}
$$


This leads to

$$
\begin{aligned}
J(x) & =\left(\frac{2}{\alpha+\beta}\right)^{p+1} \sum_{\lambda=0}^{k} \sum_{\mu=0}^{k^{\prime}} \frac{(-)^{\mu} \gamma^{\lambda+\mu}}{\lambda ! \mu !} \\
& *\left\{\int_{0}^{\infty} y^{p+\lambda+\mu} e^{-y} L_{n+k}^{n+\lambda}(y) L_{n^{\prime}+k^{\prime}}^{n^{\prime}+\mu}(y) d y\right\} .
\end{aligned}
$$

The integral in (D.23) is evaluated using the generating function

$$
\sum_{k=0}^{\infty} L_{n+k}^{n}(x) \frac{t^{k}}{(n+k) !}=\frac{(-)^{n}}{(1-t)^{n+1}} \exp \left(-\frac{t}{t-1} x\right) t^{n}
$$

to obtain

$$
\begin{aligned}
Q(x) & =\int_{0}^{\infty} x^{p} e^{-x} L_{n+k}^{n}(x) L_{n^{\prime}+k^{\prime}}^{k^{\prime}}(x) d x \\
& =p !(n+k) !\left(n^{\prime}+k^{\prime}\right) ! \sum_{\tau=0}^{\leq k}(-1)^{C}\left(\begin{array}{c}
p-n \\
k-\tau
\end{array}\right)\left(\begin{array}{c}
p-n^{\prime} \\
k^{\prime}-\tau
\end{array}\right)\left(\begin{array}{c}
-p-1 \\
\tau
\end{array}\right)(\mathrm{D} .
\end{aligned}
$$

With (D.24)

$$
\begin{aligned}
J(x) & =\left(\frac{2}{\alpha+\beta}\right)^{p+1}(n+k) !\left(n^{\prime}+k^{\prime}\right) ! \sum_{\lambda=0}^{k} \sum_{\mu=0}^{k^{\prime}} \frac{(-)^{\mu} \gamma^{\lambda+\mu}}{\lambda ! \mu !}(p+\lambda+\mu) ! \\
& * \sum_{\tau=0}^{\leq k-\lambda}(-1)^{C}\left(\begin{array}{c}
p+\mu-n \\
k-\lambda-\tau
\end{array}\right)\left(\begin{array}{c}
p+\lambda-n^{\prime} \\
k^{\prime}-\mu-\tau
\end{array}\right)\left(\begin{array}{c}
-p-\lambda-\mu-1 \\
\tau
\end{array}\right)(\mathrm{D}
\end{aligned}
$$

where $C=n+n^{\prime}+k+k^{\prime}+\tau$. For other methods of solution of integrals over generalised Laguerre polynomials see Gordon [Go 30], Erdélyi [Er 36] and Mayr [Ma 35].

\section{D.3 Sturmian Functions}

Although the eigenfunctions of the Schrödinger equation form a complete set, the fact that the continuum forms part of that set often reduces the completeness property to formal importance only. The continuum terms are then usually ignored because the ensuing equations are impossible to deal with. This is the case with the discrete Coulomb basis given in (D.6). This basis is incomplete in the sense that the positive energy of the atom (the continuum part) has been ignored [Ed 73]. It also leads to a matrix equation that is impractical to implement as can be seen with the radial integral in (6.18). To overcome these drawbacks a basis of Sturmian functions is introduced which can represent the Coulomb functions efficiently at small $r$.

To define a complete basis, the radial functions are represented in terms of a suitable set of orthonormal polynomials defined in the interval $(0, \infty)$. Such functions 
have in general no direct physical significance but they have several practical advantages. The Sturmian functions are then defined as the nontrivial normalised solutions of $[$ Ro 70$]$

$$
\left[\frac{d^{2}}{d r^{2}}-\frac{l(l+1)}{r^{2}}+E_{0}-\alpha_{n l} V(r)\right] S_{n l}(r)=0
$$

such that

$$
S_{n l}(0)=0 ; \quad S_{n l}(\infty) \rightarrow 0 .
$$

Here $n=1,2, \ldots$ and $l=0,1, \ldots, n-1$. Equation (D.26) can be considered as a kind of Schrödinger equation, where, for a fixed total negative energy $E_{0}$ and angular momentum $l$, the coupling constants $\alpha_{n l}$ are to be found such that (D.27) is satisfied. In physical terms the potential energy is being increased as $\alpha_{n l}$ increases (it is assumed that $V(r)$ is attractive) and the kinetic energy must therefore also increase to keep $E_{0}$ constant. Therefore the number of oscillations of the Sturmian function increases, which permits the possibility of orthogonality which reads

$$
\int d r S_{n l}(r) V(r) S_{n^{\prime} l}(r)=-\delta_{n n^{\prime}} ; \quad V(r) \text { negative. }
$$

The most widely used Sturmian functions are those generated by the Coulomb potential. They satisfy

$$
\left[-\frac{d^{2}}{d r^{2}}+\frac{l(l+1)}{r^{2}}-E_{0}-\frac{2 \alpha_{n l}}{r}\right] S_{n l}(r)=0
$$

with units of length and energy the Bohr radius and the Rydberg respectively. The orthogonality property is such that

$$
\int_{0}^{\infty} d r S_{n l}(r) \frac{2}{r} S_{n^{\prime} l}(r)=\delta_{n n^{\prime}}
$$

By comparing (D.29) with that for the Coulomb function

$$
\left[-\frac{d^{2}}{d r^{2}}+\frac{l(l+1)}{r^{2}}+\frac{1}{n^{2}}-\frac{2}{r}\right] R_{n l}(r)=0
$$

with $R_{n l}$ given in (D.7), it is seen that

$$
S_{n, l}(r)=\sqrt{\frac{(n-l-1) !}{2(n+l) !}}(\zeta r)^{l+1} e^{-\zeta r / 2} L_{n-l-1}^{2 l+1}(\zeta r)
$$

with $\alpha_{n l}=\zeta n$.

Because almost all bound-state hydrogenic wave functions are close to zero energy, they suffer from the peculiarity that the innermost zeros of the functions are insensitive to the principle quantum number $n$. This means that as $n$ becomes very large, the first zero approaches a value of 1.88 Bohr radii and the region between the origin 
Figure D.1: The $l=0$ Sturmian functions for $n=1,2,3$ and 4 . Unlike hydrogen functions, the first node continues to move closer to the origin with increasing $n$ (from [Ro 70]).

and the first zero is never traversed. This is due to the incompleteness of the Coulomb set and the continuum part is needed to analise this region. Because of (D.27), the Sturmian basis set does traverse this region. The first few Sturmian functions are shown in Fig. (D.1).

The matrix elements for arbitrary powers of $r$ can now be obtained in closed analytic form. The parameter $\zeta$ may assume any positive real value without affecting completeness or orthogonality properties. Variation in $\zeta$ can be used as a check on convergence. When $\zeta=2 / n$ the functions $S_{n l}$ coincides, up to normalisation, with the hydrogenic radial wave functions for the zero-field degenerate manifold of states with fixed principle quantum number $n$ [CT 82].

The full eigenfunctions used can now be written as

$$
|n, l, m\rangle=S_{n, l} Y_{l, m}
$$

which leads to a generalised eigenvalue problem for $H$ in (D.5) which can be solved without complications. To examine a particular region of the spectrum, one should choose $\zeta$ to correspond to a value of $n$ appropriate to the given energy in the absence of a field. If [CT 82]

$$
\zeta=\frac{2}{n^{*}} ; \quad n^{*}=1,2, \ldots, n
$$

the eigenvalues will be accurate in the vicinity of $n^{*}$. Edmonds used a value of $n=50$ and $n^{*}=1$ so that $\zeta=2$ which resulted in the correct first few energy levels for the ordinary Hydrogen atom [Ed 73]. The Sturmian functions give highly accurate energy values in the chosen spectral range. 


\section{D.4 Matrix Elements Using Sturmian Functions}

Because the Sturmian functions in (D.32) lead to a generalised eigenvalue problem for the Hydrogen atom in a magnetic field, Wunner et al proposed the following orthonormal and complete set of Sturmian functions ${ }^{1}$ [WKR 86]

$$
G_{n l}^{(\zeta)}(r)=\zeta^{\frac{3}{2}} \sqrt{\frac{(n-l-1) !}{(n+l+1) !}} e^{-\zeta r / 2}(\zeta r)^{l} L_{n-l-1}^{2 l+2}(\zeta r)
$$

This basis leads to an ordinary eigenvalue problem. In this basis the radial matrix elements can be calculated analytically. Note however that the ordinary Hydrogen atom, $H_{0}$, is not diagonal in this basis.

\section{D.4.1 The Radial Matrix Elements}

The radial matrix elements are now, with the help of (D.21), calculated to be in closed analytical form. Let $G_{n l}^{(\zeta)} \equiv|n, l\rangle$ and then

$$
\begin{aligned}
\left\langle l^{\prime}, n^{\prime}\left|r^{q}\right| n, l\right\rangle & =\zeta^{3+l+l^{\prime}} \sqrt{\frac{(n-l-1) !\left(n^{\prime}-l^{\prime}-1\right) !}{(n+l+1) !\left(n^{\prime}+l^{\prime}+1\right) !}} \\
& *\left\{\int_{0}^{\infty} r^{2+q+l+l^{\prime}} e^{-\zeta r} L_{n-l-1}^{2 l+2}(\zeta r) L_{n^{\prime}-l^{\prime}-1}^{2 l^{\prime}-2}(\zeta r) d r\right\} \\
& =\zeta^{-q} \sqrt{\frac{(n-l-1) !\left(n^{\prime}-l^{\prime}-1\right) !}{(n+l+1) !\left(n^{\prime}+l^{\prime}+1\right) !}}\left(l+l^{\prime}+p+2\right) !\left\{\begin{array}{c}
\leq_{n^{\prime}-l^{\prime}-1}^{n-l-1} \\
\sum_{\tau=0}
\end{array}(-1)^{C}\right. \\
& \left.*\left(\begin{array}{c}
q-l+l^{\prime} \\
n-l-1-\tau
\end{array}\right)\left(\begin{array}{c}
q+l-l^{\prime} \\
n^{\prime}-l^{\prime}-1-\tau
\end{array}\right)\left(\begin{array}{c}
-\left(q+l+l^{\prime}+3\right) \\
\tau
\end{array}\right)\right\}
\end{aligned}
$$

with $C=n+n^{\prime}-l-l^{\prime}+\tau$. For the Hydrogen atom in a strong magnetic field $q=2$. The binomial coefficients can be used to obtain the selection rule for $n$. We show this by letting $q=2$. Now if $l=l^{\prime}$ the binomial coefficients are

$$
\left(\begin{array}{c}
2 \\
n-l-1-\tau
\end{array}\right)\left(\begin{array}{c}
2 \\
n^{\prime}-l^{\prime}-1-\tau
\end{array}\right) .
$$

The binomial coefficients are only defined if

$$
\begin{aligned}
n-l-3 \leq \tau & \leq n-l-1 \\
n^{\prime}-l^{\prime}-3 \leq \tau & \leq n^{\prime}-l^{\prime}-1
\end{aligned}
$$

\footnotetext{
${ }^{1}$ Wunner et al used the radial quantum number $n$ while, here, $n$ is the principle quantum number.
} 
or ( $l$ is a constant)

$$
\begin{aligned}
n-3 \leq \tau & \leq n-1 \\
n^{\prime}-3 \leq \tau & \leq n^{\prime}-1
\end{aligned}
$$

so that

$$
\begin{aligned}
n-3 & \leq n^{\prime}-1 \\
n^{\prime}-3 & \leq n-1
\end{aligned}
$$

and $\left|n-n^{\prime}\right| \leq 2$.

As an example in using (D.36) let's calculate the matrix element when $l^{\prime}=l+2$ and $n^{\prime}=n-1$ :

$$
\begin{aligned}
\left\langle n-2, l\left|r^{2}\right| n, l\right\rangle & =\zeta^{-2} \sqrt{\frac{(n-l-1) !(n-l-3) !}{(n+l+1) !(n+l-1) !}}(2 l+4) ! \\
& *\left\{\sum_{\tau=0}(-)^{\tau}\left(\begin{array}{c}
2 \\
n-l-1-\tau
\end{array}\right)\left(\begin{array}{c}
2 \\
n-l-3-\tau
\end{array}\right)\left(\begin{array}{c}
-(2 l+5) \\
\tau
\end{array}\right)\right\}
\end{aligned}
$$

so that $n-l-3 \leq \tau \leq n-l-1$ and $n-l-5 \leq \tau \leq n-l-3$ which leads to the conclusion that $\tau=n-l-3$ only. The sum over $\tau$ is now finite and can be done analytically $^{2}$. All the radial matrix elements with $q=2$ can be calculated in the same manner and are given by $\left(H_{r}=\left\langle l^{\prime}, n^{\prime}\left|r^{2}\right| n, l\right\rangle\right)$ :

When $l^{\prime}=l$ we have

$$
\begin{array}{rll}
n^{\prime}=n-2 & : & H_{r}=\zeta^{-2} \sqrt{(n-l-2)(n-l-1)(n+l)(n+l+1)} \\
n^{\prime}=n-1 & : & H_{r}=-4 n \zeta^{-2} \sqrt{(n-l-1)(n+l+1)} \\
n^{\prime}=n & : & H_{r}=2 \zeta^{-2}[3 n(n+1)-l(l+2)] \\
n^{\prime}=n+1 & : & H_{r}=-4(n+1) \zeta^{-2} \sqrt{(n-l)(n+l+2)} \\
n^{\prime}=n+2 & : & H_{r}=\zeta^{-2} \sqrt{(n-l+1)(n-l)(n+l+2)(n+l+3)}
\end{array}
$$

and for $l^{\prime}=l+2$ we have

$$
\begin{array}{rll}
n^{\prime}=n-2 & : & H_{r}=\zeta^{-2} \sqrt{(n-l-1)(n-l-2)(n-l-3)(n-l-4)} \\
n^{\prime}=n-1 & : & H_{r}=-4 \zeta^{-2} \sqrt{(n-l-1)(n-l-2)(n-l-3)(n+l+2)} \\
n^{\prime}=n & : & H_{r}=6 \zeta^{-2} \sqrt{(n-l-1)(n-l-2)(n+l+2)(n+l+3)} \\
n^{\prime}=n+1 & : & H_{r}=-4 \zeta^{-2} \sqrt{(n-l-1)(n+l+2)(n+l+3)(n+l+4)} \\
n^{\prime}=n+2 & : & H_{r}=\zeta^{-2} \sqrt{(n+l+2)(n+l+3)(n+l+4)(n+l+5)}
\end{array}
$$

\footnotetext{
${ }^{2}$ It might happen that $\tau=n-l-2, n-l-1, n-l, n-l+1$. Here the summation is still finite (with 3 terms) and can also be done analytically.
} 
and for $l^{\prime}=l-2$ we have

$$
\begin{array}{rll}
n^{\prime}=n-2 & : \quad H_{r}=\zeta^{-2} \sqrt{(n+l-2)(n+l-1)(n+l)(n+l+1)} \\
n^{\prime}=n-1 & : \quad H_{r}=-4 \zeta^{-2} \sqrt{(n+l-1)(n+l)(n+l+1)(n-l)} \\
n^{\prime}=n & : \quad H_{r}=6 \zeta^{-2} \sqrt{(n+l)(n+l+1)(n-l)(n-l+1)} \\
n^{\prime}=n+1 & : \quad H_{r}=-4 \zeta^{-2} \sqrt{(n+l+1)(n-l)(n-l+1)(n-l+2)} \\
n^{\prime}=n+2 & : \quad H_{r}=\zeta^{-2} \sqrt{(n-l)(n-l+1)(n-l+2)(n-l+3)} .
\end{array}
$$

\section{D.4.2 The Ordinary Hydrogen Atom}

We have

$$
H_{0}=-\frac{\nabla^{2}(r)}{2}-\frac{1}{r}
$$

with

$$
\nabla^{2}(r)=\frac{d^{2}}{d r^{2}}+\frac{2}{r} \frac{d}{d r}-\frac{l(l+1)}{r^{2}}
$$

To calculate the matrix elements of the operator $H_{0}$ we need a differential equation satisfying $G_{n l}^{(\zeta)}(r)$ defined in (D.35). It is known that

$$
\left[r \frac{d^{2}}{d r^{2}}+(2 l+3-\zeta r) \frac{d}{d r}+\zeta(n-l-1)\right] L_{n-l-1}^{2 l+2}(\zeta r)=0
$$

and that

$$
r \frac{d}{d r} L_{\beta}^{\alpha}(\zeta r)=\beta L_{\beta}^{\alpha}(\zeta r)-(\beta+\alpha) L_{\beta-1}^{\alpha}(\zeta r)
$$

Now, by differentiating $G_{n l}^{(\zeta)}(r)$ twice with respect to $r$ and by using (D.42)

$$
\begin{aligned}
\left(\frac{d^{2}}{d r^{2}}+\frac{1}{r} \frac{d}{d r}\right) G_{n l}^{(\zeta)}(r) & =A e^{-\zeta r / 2} r^{l}\left[\frac{l(l+1)}{r^{2}}+\frac{\zeta^{2}}{4}-\frac{\zeta(n-l-1)}{r}\right. \\
& \left.-\frac{l}{r^{2}}-\frac{\zeta}{r}\left(l+\frac{1}{2}\right)-\frac{2}{r} \frac{d}{d r}\right] L_{n-l-1}^{2 l+2}(\zeta r)
\end{aligned}
$$

with $A$ the normalisation constant of $G_{n l}^{(\zeta)}(r)$. By reordering the terms and by using (D.43)

$$
\begin{aligned}
0= & \left\{\frac{d^{2}}{d r^{2}}+\frac{2}{r} \frac{d}{d r}-\frac{l(l+1)}{r^{2}}-\frac{\zeta^{2}}{4}+\frac{\zeta n}{r}+\frac{n-l-1}{r}\right\} G_{n l}^{(\zeta)}(r) \\
& -\frac{1}{r^{2}} \sqrt{(n-l-1)(n+l+1)} G_{n-1, l}^{\zeta}(r) .
\end{aligned}
$$


With (D.41) $\nabla^{2}$ can be identified in (D.44) so that

$$
H_{0} G_{n l}^{(\zeta)}=\left[\frac{n-l-1}{2 r^{2}}+\frac{\zeta n}{2 r}-\frac{\zeta^{2}}{8}-\frac{1}{r}\right] G_{n l}^{(\zeta)}-\frac{1}{2 r^{2}} \sqrt{(n-l-1)(n+l+1)} G_{n-1, l}^{\zeta} .
$$

With $|n, l, m\rangle \equiv G_{n l}^{(\zeta)}(r) Y_{l m}(\theta, \phi)$, the matrix elements of $H_{0}$ are given by

$$
\begin{aligned}
\left\langle m^{\prime}, l^{\prime}, n^{\prime}\left|H_{0}\right| n, l, m\right\rangle & =\frac{1}{2}\left\{-\frac{\zeta^{2}}{4} \delta_{n^{\prime} n}+(\zeta n-2)\left\langle l^{\prime}, n^{\prime}\left|\frac{1}{r}\right| n, l\right\rangle\right. \\
& +(n-l-1)\left\langle l^{\prime}, n^{\prime}\left|\frac{1}{r^{2}}\right| n, l\right\rangle \\
& \left.-\sqrt{(n-l-1)(n+l+1)}\left\langle l^{\prime}, n^{\prime}\left|\frac{1}{r^{2}}\right| n-1, l\right\rangle\right\} \delta_{m^{\prime} m}(D)
\end{aligned}
$$

Integrals of the form $\left\langle l^{\prime}, n^{\prime}\left|1 / r^{q}\right| n, l\right\rangle(q=1,2)$ are calculated using Schrödinger's radial formula in (D.21) viz.

$$
\begin{aligned}
& \left\langle m^{\prime}, l^{\prime}, n^{\prime}\left|H_{0}\right| n, l, m\right\rangle=-\frac{\zeta^{2}}{8} \delta_{n^{\prime} n}+\frac{\zeta^{2}}{2} \sqrt{\frac{(n-l-1) !}{(n+l+1) !}} \sqrt{\frac{\left(n^{\prime}-l-1\right) !}{(n+l+1) !}} \sum_{\tau=0}^{\substack{n-l-1 \\
n^{\prime}-l-1}} \frac{(2 l+\tau) !}{\tau !} \\
& \text { * } \quad\left[(n-l-1)(n-l-\tau)\left(n^{\prime}-l-\tau\right)+(n-2 / \zeta)(2 l+1+\tau)\right. \\
& \left.-(n+l+1)(n-l-1-\tau)\left(n^{\prime}-l-\tau\right)\right] \delta_{l^{\prime} l} \delta_{m^{\prime} m} \text {. }
\end{aligned}
$$

From (D.47) it is clear that $H_{0}$ is diagonal with respect to $l$ with blocks (with indices $n$ and $n^{\prime}$ ) on the diagonal. Using this basis, $H_{0}$ is reducible in $l$-subspaces. $H_{0}$ is not banded and $n, n^{\prime}=1,2, \ldots$

\section{D.5 Solving the Schrödinger Equation}

This section describes the method used by Wintgen and Friedrich to obtain the spectrum for the Hydrogen atom in a magnetic field. This method preserves the classical phase space dynamics where they showed that the quantum system also depends on the scaled energy $\varepsilon$ only.

They used the Hamiltonian in (6.2) which is given in cylindrical coordinates

$$
H=\frac{p_{\rho}^{2}}{2}+\frac{p_{z}^{2}}{2}-\frac{1}{\sqrt{\rho^{2}+z^{2}}}+\frac{l_{z}^{2}}{2 \rho^{2}}+\frac{1}{8} \gamma^{2} \rho^{2} .
$$

It is of practical advantage to introduce the semiparabolic coordinates $(\mu, \nu)$ given in (6.7) so that $H$ can be transformed and the Scrödinger equations reads $\left(l_{z}=0\right)$

$$
\left[\Delta_{\mu}+\Delta_{\nu}+E\left(\mu^{2}+\nu^{2}\right)-\frac{1}{4} \nu^{2} \mu^{2}\left(\nu^{2}+\mu^{2}\right)+4\right] \psi(\mu, \nu)=0 .
$$


$\Delta_{\mu}$ and $\Delta_{\nu}$ are the Laplacians for the radial parts $\mu$ and $\nu$, e.g.

$$
\Delta_{\mu}=\frac{1}{\mu} \frac{\partial}{\partial \mu} \mu \frac{\partial}{\partial \mu} .
$$

For fixed values of $E<0$ the first two terms of (D.48) represent two decoupled twodimensional harmonic oscillators, the third term is a sixth order perturbation and the last term is simply a numerical constant.

The Schrödinger equation (D.48) is solved by taking matrix elements in a basis of (parity-projected) harmonic oscillator states characterised by a given oscillator width b. Equation (D.48) thus becomes

$$
\left[-\frac{1}{b^{2}} \tilde{T}+E b^{2} \tilde{V}_{\text {но }}-\frac{1}{4} \gamma^{2} b^{6} \tilde{V}_{\mathrm{B}}+4\right] \psi=0
$$

where $\tilde{T}, \tilde{V}_{\text {Hо }}$ and $\tilde{V}_{\text {B }}$ are all sparse banded matrices of the operators $-\Delta_{\mu}-\Delta_{\nu}, \mu^{2}+\nu^{2}$ and $\mu^{4} \nu^{2}+\mu^{2} \nu^{4}$ respectively. The elements of these matrices are pure dimensionless numbers independent of $E, b$ and $\gamma$.

Equation (D.49) is best solved by taking $b=\gamma^{-1 / 3}$ so that

$$
\left(E \gamma^{-2 / 3} \tilde{V}_{\mathrm{HO}}-\frac{1}{4} \tilde{V}_{\mathrm{B}}+4\right) \psi=\gamma^{2 / 3} \tilde{T} \psi
$$

For fixed values of $E \gamma^{-2 / 3}=\varepsilon$, (D.50) represents a generalised eigenvalue problem for the field strengths $\gamma^{2 / 3}$. This method generates spectra, not for fixed magnetic fields $\gamma$, but along the hyperbolic lines of the constant scaled energy $\varepsilon$ [WF 87]. As described in $§ 6.2 .2$, the structure of the classical phase space is constant along these lines so that a direct comparison between the classical and quantal behaviour is possible.

By keeping $\varepsilon$ constant, but by changing $E$ and $\gamma$, we are able to explore different regions of the quantal spectrum without changing the classical dynamics. At small values of $|E|$ and $\gamma$ the quantum spectrum is very dense, whereas it is very sparse for large $|E|$ and $\gamma$. The region close to $E=0$ and $\gamma=0$ corresponds to a small Planck's constant, $\hbar$, in comparison to classical typical actions, whereas $\hbar$ becomes larger in comparison with typical classical actions as we move along lines of constant scaled energy towards larger field strengths. This can be expressed by the commutation relations of the scaled quantal operators associated with the scaled classical variables (6.3), e.g. [Fr 88]

$$
\left[\tilde{p}_{z}, \tilde{z}\right]=\gamma^{1 / 3}\left[z, p_{z}\right]=i \gamma^{1 / 3} \hbar .
$$

This enables one to study the semi-classical limit by keeping $\varepsilon$ fixed and by decreasing the magnetic field strength. 


\section{Appendix E}

\section{Spectrum for a One Dimensional Chaotic System}

In this Appendix we describe two methods for calculating the spectrum of the model proposed in Chapter 7. The first method involves solving the Schrödinger equation directly and the second is based on a matrix representation of the Hamiltonian and its subsequent diagonalisation. The last section focuses on the problem of numerical accuracy that has to be adhered to.

\section{E.1 Schrödinger Method}

By taking the general solution of the $l$-th interval as

$$
\psi_{l}=A_{l} \cos k x+B_{l} \sin k x
$$

we find, for $x \in\left[-\frac{1}{6}, \frac{1}{6}\right]$, the values $A_{1}=1$ and $B_{1}=0$. The coefficients $A_{l}$ and $B_{l}$ in the adjacent intervals are found from the requirement that the wave function be continuous and its derivative has a jump of $\lambda \psi\left(x_{l}\right)$ at the barrier potentials. This establishes a linear relationship between the coefficients in adjacent intervals. For the interval next to $\left[-\frac{1}{6}, \frac{1}{6}\right]$ we obtain

$$
\left(\begin{array}{l}
A_{2} \\
B_{2}
\end{array}\right)=\left(\begin{array}{ll}
\left(1-\alpha \sin 2 k x_{2}\right) & -\alpha\left(1-\cos k x_{2}\right) \\
\alpha\left(1+\cos 2 k x_{2}\right) & \left(1+\alpha \cos k x_{2}\right)
\end{array}\right)\left(\begin{array}{l}
1 \\
0
\end{array}\right)
$$

and for the $l$ th interval we have

$$
\left(\begin{array}{l}
A_{l} \\
B_{l}
\end{array}\right)=\prod_{i=2}^{l}\left(\begin{array}{cc}
\left(1-\alpha \sin 2 k x_{i}\right) & -\alpha\left(1-\cos k x_{i}\right) \\
\alpha\left(1+\cos 2 k x_{i}\right) & \left(1+\alpha \cos k x_{i}\right)
\end{array}\right)\left(\begin{array}{l}
1 \\
0
\end{array}\right)
$$

where $\alpha=\lambda / 2 k$. Here the matrix product runs over the strictly ordered Cantor set in one direction, since the individual matrices do no commute. For symmetry reasons we can choose $x_{j}>0$, i.e. the product runs from $x_{2}=1 / 6$ to $x_{\infty}=1 / 2$. 
The spectrum is obtained by making use of the boundary condition

$$
A_{\infty} \cos \left(\frac{k}{2}\right)+B_{\infty} \sin \left(\frac{k}{2}\right)=0
$$

which ensures that the wave function vanishes at the boundary of the square well, i.e. $x_{\infty}=1 / 2$. The spectrum is calculated numerically by finding the values $k$ where (E.3) vanishes for $0 \leq \lambda \leq 4000$ and by putting $k_{n}=\sqrt{E_{n}}$ [HS 89].

\section{E.2 Matrix Diagonalisation}

To obtain a matrix representation of the underlying Hamiltonian we choose as a basis in Hilbert space $L^{2}\left[-\frac{1}{2}, \frac{1}{2}\right]$, the complete set of the symmetric wave functions of the unperturbed $(\lambda=0)$ problem i.e. the ordinary square well wave functions given by

$$
\begin{aligned}
& |n\rangle^{(+)}=\sqrt{2} \cos n \pi x, n \text { odd (pos parity) } \\
& |k\rangle^{(-)}=\sqrt{2} \sin k \pi x, k \text { even (neg parity). }
\end{aligned}
$$

The interaction matrix elements (positive parity) are then given by

$$
\left\langle m\left|H_{\text {int }}\right| n\right\rangle=2 \lambda \sum_{\{j\}} \cos n \pi x_{j} \cos m \pi x_{j}
$$

where the sums run over all the points in the Cantor set.

The sums which run over $2^{s}$ terms for the $s$-th generation of the partitioning of the interval can be rewritten essentially as a product of $s$ factors by using the expression

$$
\begin{aligned}
\sum_{\{j\}>0} \exp \left(i \pi q x_{j}\right) & =\exp \left(\frac{i \pi q}{6}\right)\left\{\prod_{r=2}^{s}\left[1+\exp \left(\frac{2 i \pi q}{3^{r}}\right)\right]\right. \\
& \left.+\sum_{v=0}^{s-2} \prod_{r=2}^{s-v-1}\left[1+\exp \left(\frac{2 i \pi q}{3^{r}}\right)\right] \exp \left(\frac{i \pi q}{3^{s-v}}\right)\right\}
\end{aligned}
$$

where the sum is extended over the positive points of the Cantor set and $q=n+m$ or $q=n-m$ [HS 89], [HK 90a]. The sums in (E.6) and (E.7) diverge if $s \rightarrow \infty$. This reflects the fact that the strength $\lambda$ must go to zero when infinitely many $\delta$-functions are used. To get finite values for the sums we define a new effective interaction strength parameter $\mu$ where $\lambda=\mu 2^{-s}$. This reflects the condition that $\lambda \rightarrow 0$ when $s \rightarrow \infty$.

Denoting the right hand side of (E.7) by $Z_{s}(q)$, we obtain the matrix representation of the interaction Hamiltonian as

$$
\begin{aligned}
\left\langle m\left|H_{i n t}^{(+)}\right| n\right\rangle & =\lim _{s \rightarrow \infty} 2^{-s} \operatorname{Re}\left[Z_{s}(m+n)+Z_{s}(m-n)\right] \\
n, m & =1,3,5, \ldots
\end{aligned}
$$


The full Hamiltonian matrix to be diagonalised is therefore

$$
H_{m, n}=m^{2} \pi^{2} \delta_{m, n}+\mu\left\langle m\left|H_{i n t}\right| n\right\rangle, m=1,3,5, \ldots
$$

The spectrum is obtained as a function of $\mu$ by diagonalising $H_{m, n}$. The matrix is essentially sparse, yet the off-diagonal elements never tend to zero the further away from the diagonal.

\section{E.3 Numerical Accuracy}

The sums and products in (E.7) might be very large resulting in an overflow problem if computer accuracy is not taken into account. The number of terms in the products and sums can be reduced considerably by noting that the exponential factors tend to unity very quickly when $s$ is increased. For an average double precision calculation the accuracy is roughly $10^{-20}$. We then have

$$
\left(1+\exp \frac{2 i \pi q}{3^{r}}\right) \approx 1+\frac{1}{3^{r}} 2 i \pi q+\ldots
$$

with $r=2,3, \ldots, s$. This leads to

$$
Q=\frac{2 \pi q}{3^{r}} \ll 1 ; \quad s \rightarrow \infty
$$

where, numerically, we want $Q$ to be as small as possible so that

$$
\frac{2 \pi q}{3^{r}} \approx 10^{-20} ; \quad s \rightarrow \infty
$$

Taking the logarithm on both sides leads to the maximum $s$ that can be chosen for this particular accuracy e.g.

$$
s_{\max }=44+\ln q .
$$

\section{E.4 Expansion Coefficients: Off-Center Interval}

In $\S 7.5$ the expansion coefficients for the center interval were calculated. We quote here the expressions for an off-center interval. Let $x \in[5 / 18,1 / 18]$ and the wave functions are given by

$$
\begin{aligned}
\Psi_{n}^{(+)} & =T_{n} \cos k_{n} \pi x+U_{n} \sin k_{n} \pi x \\
\Psi_{k}^{(-)} & =V_{k} \sin \bar{k}_{k} \pi x+W_{k} \cos \bar{k}_{k} \pi x
\end{aligned}
$$

where $T_{n}, U_{n}$ and $V_{k}, W_{n}$ are the normalisation constants (amplitudes) and $k_{n}$ and $\bar{k}_{k}$ are the wave numbers. 
We again expand these wave functions in terms of the bases given in (E.4) and (E.5) and go through the same procedure as described in $\S 7.5$ to obtain

$$
\begin{aligned}
& c_{n}=\frac{4}{\pi \sqrt{D}}\left(\sum_{k}\left[\alpha_{k n} \cos (2 k-1) \pi x_{0} \frac{\cos [(2 k-1) \pi / 2 D]}{1-((2 k-1) / D)^{2}}\right]\right) \\
& d_{n}=\frac{4}{\pi \sqrt{D}}\left(\sum_{j}\left[\sin 2 j \pi x_{0} \frac{\cos (\pi j / D)}{1-(2 j / D)^{2}}\right]\right) .
\end{aligned}
$$

The same conclusions as in $\S 7.5$ can also be drawn from this interval - the behaviour of the system will be the same throughout all of the intervals.

\section{E.5 Expectation Value of $x^{2}$}

In this section we give the full derivation of the formula for the spreading of a particle inside the center interval which was listed in (7.18).

$\left\langle\psi_{n}|x| \psi_{n}\right\rangle$ is the expectation value of the position $x$. Let $x$ be in the center interval where we can have $x=0$ so that from (7.14) we have

$$
N_{n}=\sqrt{2} \sum_{l} \alpha_{n}^{l}
$$

with $N_{n}$ the amplitudes of the wave functions used in $\S 7.5$. Here $\alpha_{n}^{l}$ is the $n$-th eigenvector of the matrix hamiltonian in (7.6). Let the wave functions be

$$
\psi_{n}=N_{n} \cos k_{n} \pi x ; \quad x \in[-1 / 6,1 / 6]
$$

and the basis is

$$
\phi_{l}=\sqrt{2} \cos (2 l-1) \pi x ; \quad x \in[-1 / 2,1 / 2] .
$$

Now, expand $\psi_{n}$ according to the basis in (E.15) such that

$$
\psi_{n}(x)=\sum_{l} \alpha_{n}^{l} \phi_{l}(x), \quad x \in[-1 / 2,1 / 2]
$$

with

$$
\alpha_{n}^{l}=\left\langle\phi_{l}(x) \mid \psi_{n}(x)\right\rangle=\int_{-\frac{1}{6}}^{\frac{1}{6}} \psi_{n}(x) \phi_{l}^{*}(x) d x .
$$

To simplify our notation we work in the number representation so that

$$
\left|\psi_{n}(x)\right\rangle=|n\rangle
$$

where

$$
|0\rangle=\sqrt{2} \delta_{n l} \cos (2 l-1) \pi=\phi_{l}(x)
$$


The state where $|n\rangle=|0\rangle$ is thus the state where $\mu=0$ (the ordinary empty potential well) and the total wave functions $\psi_{n}(x)$ are the same as the basis wave functions $\phi_{l}(x)$ and we have

$$
\sum_{l} \alpha_{n}^{l}=\delta_{n l}
$$

The overlap of the basis wave functions with the total wave functions inside the center interval is then $\langle 0 \mid n\rangle$ and we have from (E.17)

$$
\alpha_{n}^{l}=\langle 0 \mid n\rangle
$$

so that

$$
N_{n}=\sqrt{2} \sum_{l}\langle 0 \mid n\rangle .
$$

The distribution of the particle inside the well is

$$
\begin{aligned}
\left\langle n\left|x^{2}(\mu)\right| n\right\rangle & =\sum_{l=1}^{\infty} \sum_{k=1}^{\infty} \alpha_{n}^{k}\left\langle 0\left|x^{2}\right| 0\right\rangle \alpha_{n}^{l} \\
& =2 \sum_{l} \sum_{k} \alpha_{n}^{k} \alpha_{n}^{l} \int_{-\frac{1}{2}}^{\frac{1}{2}}[\cos (2 k-1) \pi x] x^{2}(\mu)[\cos (2 l-1) \pi x] d x
\end{aligned}
$$

Let

$$
\begin{aligned}
(2 k-1) \pi & =a \\
(2 l-1) \pi & =b
\end{aligned}
$$

and because of the evenness of the integrand we have

$$
\left\langle n\left|x^{2}\right| n\right\rangle=4 \sum_{l} \sum_{k} \alpha_{n}^{k} \alpha_{n}^{l} A
$$

with

$$
A=\int_{0}^{\frac{1}{2}} \cos a x\left(x^{2} \cos b x\right) d x
$$

Indefinite integration of (E.20) leads to

$$
\begin{aligned}
A= & \frac{1}{\gamma^{3} \eta^{3}}\left[\cos a x \cos b x\left(\eta^{3} \gamma x+\gamma^{3} \eta x\right)\right. \\
& +\cos a x \sin b x\left(-\eta^{3}\left(1-\frac{\gamma^{2} x^{2}}{2}\right)+\gamma^{3}\left(1-\frac{\eta^{2} x^{2}}{2}\right)\right) \\
& -\sin a x \cos b x\left(-\eta^{3}\left(1-\frac{\gamma^{2} x^{2}}{2}\right)+\gamma^{3}\left(1-\frac{\eta^{3} x^{2}}{2}\right)\right) \\
& \left.\left.+\sin a x \sin b x\left(-\eta^{3} \gamma x+\gamma^{3} \eta x\right)\right)\right] ; \quad a \neq b
\end{aligned}
$$


where $\gamma=a+b$ and $\eta=a-b$. Because of the singularity in (E.21) (when $a=b$ ) we use the rule by L'Hospital $^{1}$ so that

$$
\begin{aligned}
\lim _{\eta \rightarrow 0} A(\gamma, n)=A(\gamma, \eta)_{a=b}= & \frac{1}{12 a^{3}}\left[3\left(2 x^{2} a^{2}-1\right) \sin a x \cos a x\right. \\
& \left.+3 a x\left(1-2 \sin ^{2} a x\right)+2 a^{3} x^{3}\right] .
\end{aligned}
$$

Substitution of the boundary conditions at $x=0$ and $x=1 / 2$ into (E.21) leads to the distribution of the particle inside the center interval

$$
\left\langle n\left|x^{2}\right| n\right\rangle=4 \begin{cases}\sum_{k} \sum_{l} \alpha_{n}^{k} \alpha_{n}^{l}\left\{\frac{4}{(a+b)^{3}(a-b)^{3}}\left[\sin \frac{a}{2} \sin \frac{b}{2}\left(\frac{a^{3} b}{2}-\frac{a b^{3}}{2}\right)\right]\right\} & a \neq b \\ \sum_{l} \alpha_{n}^{l} \alpha_{n}^{l}\left\{\frac{1}{48}-\frac{1}{8 a^{2}}\right\} & a=b .\end{cases}
$$

The overlap of $x^{2}$ and the wave functions is in the form of a parabola around the origin. The amplitudes of the wave functions, on the other hand, tend to zero at the boundary. A comparison of the amplitudes of the wave functions $N_{n}$ with the overlap shows that if $N_{n}$ is small, $\left\langle n\left|x^{2}\right| n\right\rangle$ is large and if $N_{n}$ is large, $\left\langle n\left|x^{2}\right| n\right\rangle$ is small, but if $x=n=0$ we have

$$
N_{n}=\sqrt{2}\left\langle n\left|x^{2}\right| n\right\rangle .
$$

If $n=1$ and $x=0$ we have $\left\langle n\left|x^{2}\right| n\right\rangle<N_{n}$. æ

\footnotetext{
${ }^{1}$ This limiting case can also be calculated with $A_{a=b}=\int x^{2} \cos ^{2} a x d x=\frac{1}{2} \int x^{2} \cos 2 a x d x+$ $\frac{1}{2} \int x^{2} d x$.
} 


\section{Bibliography}

[Ar 80] V. I. Arnol'd, Mathematical Methods of classical Mechanics, Springer New York 1980

[AS 65] M. Abramowitz and I. A. Stegun (eds), Handbook of Mathematical Functions, Dover Publications, New York 1965

[Ba 90] H. Bai-Lin, Chaos II, World Scientific (1990)

[BA 92] D. Biswas, M. Azan, Q. V. Lawande and S. V. Lawande, J. Phys. A, 25, L297 (1992)

[Be 81] M. V. Berry, Ann. Phys. (NY), 131, 163 (1981)

[Be 85] M. V. Berry, Proc. Roy. Soc. London, Ser. A 400, 229 (1985)

[BF 81] T. A. Brody, J. Flores, J. B. French, P. A. Mello, A. Pandey and S. S. M. Wong, Rev. Mod. Phys. 53, 385 (1981)

[BG 75] O. Bohigas and M.-J. Giannoni, Annals of Physics 89, 393 (1975)

[BG 84] O. Bohigas and M.-J. Giannoni, Chaotic motion and random matrix theories in Mathematical and Computational Methods in Nuclear Physics, eds. J. S. Dehesa, J. M. J. Gomez and A. Polls, Lecture Notes in Physics 209, 1 (Springer Verlag 1984)

[BGS 84] O. Bohigas, M.-J. Giannoni and C. Schmit, Phys. Rev. Lett. 52, 1 (1984)

[BGS 86] O. Bohigas, M.-J. Giannoni and C. Schmit, Spectral fluctuations of classically chaotic quantum systems in Mathematical and Computational Methods in Nuclear Physics, eds. T. H. Seligman and H. Nishioka, Lecture Notes in Physics 263, Springer (1986)

[BHS 74] C. M. Bender, H. J. Happ and B. Svetitsky, Phys. Rev. D 9, 2324 (1974)

[Bo 91] O. Bohigas in Proceedings of the 1989 Les Houches Summer School on Chaos and Quantum Physics, Elsevier (1991) 
[Bo 92] E.B. Bogomolny, in Proceedings of the 1991 Varenna Summer School on Quantum Chaos, Italian Physical Society (to be published)

E. B. Bogomolny, Physica D, 31, 169 (1988)

[Bo 92a] O. Bohigas, Preprint (submitted to Physics Reports)

[Br 73] T. A. Brody, Nuovo Cim. 7, 482 (1973)

[BR 86] M. V. Berry and M. Robnik, J. Phys. A 19, 649 (1986)

[BS 57] H. A. Bethe and E. E. Salpeter, Quantum Mechanics of One- and Twoelectron Atoms, Springer-Verlag (1957)

[BT 77] M. V. Berry and M. Tabor, Proc. R. Soc. London Ser. A356, 375 (1977)

[BW 69] C. M. Bender and T. T. Wu, Phys. Rev. 184, 1231 (1969)

C. M. Bender and T. T. Wu, Phys. Rev. D7, 1620 (1973)

[Ca 85] G. Casati, Editor, Chaotic Behaviour in Quantum Systems, Plenum Press, New York (1985)

[Ca 88] G. Casati in Nonlinear Evolution and Chaotic Phenomena, eds. G. Gallavotti and P. F. Zweifel, NATO ASI Series B, 176, Plenum Press, New York (1988)

[CCG 85] G. Casati, B. V. Chirikov and I. Guaneri, Phys. Rev Lett. 54, 1350 (1985)

[CG 83] H. S. Camarada and P.D. Georgopulos, Phys. Rev. Lett. 50, 492 (1983)

[CGR 90] E. Caurier, B. Grammaticos and A. Ramani, J. Phys. A, 23, 4903 (1990)

[Ch 84] C. Chatfield, The Analysis of Time Series: An Introduction-Third Edition, Chapman and Hall (1984)

[CIS 88] B.V. Chirikov, F.M. Izrailev and D.L. Shepelyansky, Physica D, 33, 77 (1988)

[Co 74] P. M. Cohen, Algebra - Vol 1, John Wiley and and Sons (1974)

[CP 84] F. J. Carnegie and L. Percival, J. Math. Phys. 17, 701 (1984)

[CT 82] C. W. Clark and K. T. Taylor, J. Phys. B 15, 1175 (1982)

[De 89] R. L. Devaney, An Introduction to Chaotic Dynamical Systems - Second Edition, Addison-Wesley (1989)

[DG 84] D. Delande and J. C. Gay, J. Phys. B 17, L335 (1984)

[DG 86] D. Delande and J. C. Gay, J. Phys. B 19, L173 (1986) 
[DM 63] F. J. Dyson and M. L. Mehta, J. Math. Phys. 4, 701 (1963)

[DR 90] P. Dahlqvist and G. Russberg, Phys. Rev. Lett. 65, 2837 (1990)

[DR 91] P. Dahlqvist and G. Russberg, J. Phys. A, 24, 4763 (1991)

[Dy 62] F. J. Dyson, J. Math. Phys. 3, 140 (1962)

F. J. Dyson and M. L. Mehta, ibid. 4, 701 (1963) (both reprinted in [Po 65])

[Dy 72] F. J. Dyson, J. Math. Phys. 13, 90 (1972) (reprinted in [Po 65])

[Ec 88] B. Eckhardt, Phys. Rep. 163, 205 (1988)

[Ed 57] A. R. Edmonds, Angular Momentum in Quantum Mechanics, Princeton University Press (1957)

[Ed 73] A. R. Edmonds, J. Phys. B 6, 1603 (1973)

[EHP 89] B. Eckhardt, G. Hose and E Pollak, Phys. Rev. A 39, 3776 (1989)

[Ei 17] A. Einstein, Verhandlungen der Deutschen Physikalische Gesellschaft, 19, 82 (1917)

[En 72] M. J. Englefeld, Group Theory and the Coulomb Problem, Wiley-Interscience (1972)

[Er 36] A. Erdélyi, Math. Zeitsch. 40, 45 (1936)

[ER 91] G. S. Ezra, K. Richter, G. Tanner and D. Wintgen, J. Phys. B, 24, L413 (1991)

[EW 90] B. Eckhardt and D. Wintgen, J. Phys. B, 23, 355 (1990)

[Fi 83] M. E. Fisher in Critical Phenomena, ed. F. J. Hahne, Lecture Notes in Physics 186, Springer (1983)

[Fi 92] S. Fishman, in Proceedings of the 1991 Varenna Summer School on Quantum Chaos, Italian Physical Society (to be Published)

[FMP 78] J. B. French, P. A. Mello and A. Pandey, Ann. Phys. (NY), 113, 277 (1978) (paper II of series)

A. Pandey, ibid. 119, 170 (1979) (paper III of series)

J. B. French, V. K. B. Kota, A. Pandey and S. Tomsovic, ibid. 181, 198 (1988) (paper V of the series)

[Fr 82] H. Friedrich, Phys. Rev. A 26, 1827 (1982) 
[Fr 88] H. Friedrich in Atomic Spectra and Collisions in External Fields, eds. K. T. Taylor, M. H. Nayfeh and C. W. Clark, Physics of Atoms and Molecules, Series eds. P. G. Burke and H. Kleinpoppen, Plenum Press (1988)

[FW 89] H. Friedrich and D. Wintgen, Phys. Rep. 183, 37 (1989)

[Ga 77] R. H. Garstang, Rep. Prog. Phys. 40, 105 (1977)

[GH 86] M. Z. I. Gering and W. D. Heiss, Phys. Rev. D 33, 1980 (1986)

[Gl 87] J. Gleick, Chaos: Making a New Science, Viking, New York (1987)

[Go 30] W. Gordon, Ann. d. Phys 5, 1031 (1930)

[Gu 85] M. Gutzwiller in Chaotic Behaviour in Quantum Systems, ed. G. Casati, NATO ASI Series B, 120, Plenum Press, New York (1985)

[Gu 71] M. C. Gutzwiller, J. Math. Phys. 12, 343 (1971)

[Gu 90] M. C. Gutzwiller, Chaos in Classical and Quantum Mechanics, SpringerVerlag (1990)

[GW 90] T. Guhr and H. A. Weidenmüller, Ann. Phys. (NY), 199, 412 (1990)

[HC 92] W. D. Heiss and J. C. H. Chiang, Phys. Rev. A 47, 2533 (1993)

[He 84] E. J. Heller, Phys. Rev. Lett. 53, 1515 (1984)

S. Tomsovic and E. J. Heller, Phys. Rev. Lett. 67, 664 (1991)

[He 88] W. D. Heiss, Z. Phys. A 329, 133 (1988)

[HK 88a] W. D. Heiss and A. A. Kotzé, S. Afr. J. Phys. 11, 26 (1988)

[HK 88b] W. D. Heiss and A. A. Kotzé, Z. Phys. A 331, 223 (1988)

[HK 90a] W. D. Heiss and A. A. Kotzé, Z. Phys. A 335, 131 (1990)

[HK 90b] W. D. Heiss and A. A. Kotzé, Z. Phys. A 339, 223 (1990)

[HK 91] W. D. Heiss and A. A. Kotzé, Phys. Rev. A, 44, 2403 (1991)

[HL 91] F. Haake, G. Lenz, P. Seba, J. Stein, H.-J. Stöckmann and K. Zyczkowski, Phys Rev A, 44, R6161 (1991)

[HM 88] A. Holle, J. Main, G. Wiebusch, H. Rottke and K. H. Welge, Phys. Rev. Lett. 61, 161 (1988)

W. P. Reinhard, J. Phys. B, 16, L635 (1983) 
[HO 84] M. Hillary, R. F. O'Connel, M. O. Scully and E. P. Wigner, Phys. Rep. 106, $121(1984)$

[HPB 82] R. U. Haq, A. Pandey and O. Bohigas, Phys. Rev. Lett. 48, 1086 (1982)

[HS 89] W. D. Heis and W.-H. Steeb, Phys. Rev. A, 39, 6018 (1989)

[HS 90] W. D. Heiss and A. L. Sanino, J. Phys. A, 23, 1167 (1990)

[HS 91a] W. D. Heiss and A. L. Sanino, Phys. Rev. A, 43, 4159 (1991)

[HS 91b] W. D. Heiss and W.-H. Steeb, J. Math. Phys. 32, 3003 (1991)

[HT 83] G. Hose and H. S. Taylor, Phys. Rev. Lett. 51, 947 (1983)

[HW 89] A. Hönig and D. Wintgen, Phys. Rev. A 39, 5642 (1989)

[IW 90] S. Iida, H. A. Weidenmüller and J. A. Zuk, Ann. Phys. (NY), 200, 219 (1990)

[Iz 90] F. M. Izrailev, Phys. Rep. 196, 299 (1990)

[JP 92] G. Jona-Lasinio, C. Presilla, and F. Capasso, Phys. Rev. Lett. 68, 2269 (1992)

[JS 91] R. V. Jensen, S. M. Susskind and M. M. Sanders, Phys. Rep. 201, 1 (1991)

[Ka 66] T. Kato Perturbation Theory of Linear Operaters, Springer (1966)

[KH 92a] A. A. Kotzé and W. D. Heiss, S. Afr. J. Phys. 15, 102 (1992)

[KH 92b] A. A. Kotzé and W. D. Heiss, J. Phys. A 27, 1 (1994)

[Ki 79] J. Killingbeck, J. Phys. B 12, 25 (1979)

[Ko 88a] A. A. Kotzé MSc. Dissertation, University of the Witwaterrand, Johannesburg South Africa (1988)

[Ko 88b] A. A. Kotzé in Proceedings of the Workshop on Finite Dimensional Integrable Nonlinear Dynamical Systems, eds. P.G.L. Leach and W.-H. Steeb, World Scientific (1988)

[Ko 92] P. M. Koch in Proceedings of the Eighth South African Summer School in Theoretical Physics on Chaos and Quantum Chaos, Eds. W.D. Heiss et al, Springer-Verlag (1992)

[Ko 93] A. A. Kotzé (preprint)

[Ku 29] A. Kupper, Ann. der Phys. 86, 511 (1929) 
[LL 83] A. J. Lichtenberg and M. A. Liebermann, Regular and Stochastic Motion, Springer-Verlag, (1983)

[LZ 91] T. X. Lu, X. Z. Zhao and Z. F. Cui, Phys. Lett. A, 158, 63 (1991)

[Ma 35] K. Mayr, Math. Zeitsch. 39, 597 (1935)

[Ma 92] R. S. MacKay, in Proceedings of the 1991 Varenna Summer School on Quantum Chaos, Italian Physical Society (to be published)

[MB 88] G. E. Mitchell, E. G. Bilpuch, P. M. Endt and J. F. Shriner Jr, Phys. Rev. Lett, 61, 1473 (1988)

[Me 91] M. L. Mehta, Random Matrices - Second Edition, Academic Press New York (1991)

[MF 91] A. D. Mirlin and Y. V. Fyodorov, J. Phys. A, 24, 2273 (1991)

[MG 60] M. L. Mehta and M. Gaudin, Nucl. Phys. 18, 420 (1960)

[MK 86] D. C. Meredith and S. E. Koonin in Mathematical and Computational Methods in Nuclear Physics, eds. J. S. Dehesa, J. M. J. Gomez and A. Polls, Lecture Notes in Physics 263, Springer (1986)

[MWR 89] C. C. Martens, R. L. Waterland and W. P. Reinhardt, J. Chem. Phys. 90, $2328(1989)$

[MW 86] J. Main, G. Wiebusch, A. Holle and K. H. Welge, Phys. Rev. Lett. 57, 2789 (1986)

[MWH 86] J. Main, G. Wiebusch, A. Holle and K. H. Welge, Phys. Rev A 57, 2789 (1986)

A. Holle, J. Main, G. Wiebusch, H. Rottke and K. H. Welge, Phys. Rev. Lett. 61, 161 (1988)

[OE 78] R. K. Otnes and L. Enochson, Applied Time Series Analysis - Vol 1, John Wiley and Sons (1978)

[OH 88] P. W. O'Conner and E. J. Heller, Phys. Rev. Lett. 61, 2288 (1988)

[Oz 88] A. M. Ozorio de Almeida, Hamiltonian Systems: Chaos and Quantization, Cambridge University Press (1988)

[Pa 65] A. Papoulus, Probability, Random Variables, and Stochastic Processes, McGraw-Hill (1965) 
[Pa 90] D. Park, Classical Dynamics and its Quantum Analogues, Springer-Verlag 1990

[Pe 73] I. C. Percival, J. Phys. B, 6, L229 (1973)

[PE 81] R. A. Pullen and A. R. Edmonds, J. Phys. A, 14, L477 (1981)

[Pi 92] J.-L. Pichard, in Proceedings of the 1991 Varenna Summer School on Quantum Chaos, Italian Physical Society (to be published)

[Po 65] C. E. Porter, editor Statistical Theories of Spectra: Fluctuations, Academic New York (1965)

[Pr 76] M. B. Priestley, Spectral Analysis and Time Series - Vol. 1 (revised edition), Holden-day (1976)

[RG 91] J. M. Rost, R. Gersbacher, K. Richter, J. Briggs and Wintgen, J. Phys. B, 24, 2455 (1991)

[Ro 70] M. Rotenberg, Adv. Atom. Molec. Phys. 6, 233 (1970)

[Ro 81] M. Robnik J. Phys. A, 14, 3195 (1981)

[Ro 83] M. Robnik J. Phys. A, 16, 3971 (1984)

[Ro 84] M. Robnik J. Phys. A, 171049 (1984)

[RW 84] W. Rösner, G. Wunner, H. Herold and H. Ruder, J. Phys. B 1729 (1984)

[Sa 84] G. K. Savvidy, Nucl. Phys. B 246, 302 (1984)

[Sa 91] A. L. Sannino, Statistical Properties of Spectra and Exceptional Points, Ph.D. Thesis, Johannesburg South Africa (1991)

[Sc 26] E. Schrödinger, Ann. der Phys. 80, 483 (1926)

[Sc 84] H. G. Schuster, Deterministic Chaos: An Introduction, Physic Verlag (1984)

[Sh 88] P. E. Shanley, Ann. Phys. (NY), 186, 292 (1988)

P. E. Shanley, ibid. 186325 (1988)

[Si 70] B. Simon, Ann. Phys. (NY), 58, 76 (1970)

[Si 83] B. Simon, J. Funct. Anal. 53, 84 (1983)

[SK 89] W-H. Steeb and A. Kunick, Chaos in Dynamischen Systemen, Wissenschaftsverlag (1989) 
[SL 86] W-H. Steeb and J. A. Louw, Chaos and Quantum Chaos, World Scientific (1986)

[SLK 88] W.-H. Steeb, J. A. Louw, W. de Beer and A. A. Kotzé, Physica Scripta 37, $328(1988)$

[Sr 91] S. Sridhar, Phys. Rev. Lett. 67, 785 (1991)

[SS 39] L. I. Schiff and H. Snyder, Phys. Rev. 55, 59 (1939)

[SS 90] H.-J. Stöckmann and J. Stein, Phys. Rev. Lett. 64, 2215 (1990)

[SS 92] H.-J. Stöckmann and J. Stein, Preprint

[ST 88] W.-H. Steeb, A. J. van Tonder, C.M. Villet and C.M. Brits, Found. Phys. Lett. 1, 147 (1988)

[SVK 85] W.-H. Steeb, C. M. Villet and A. Kunick, J. Phys. A: Math. Gen. 18, 3269 (1985)

[SVZ 85] T. H. Seligman, J. J. M. Verbaarschot and M. R. Zirnbauer J. Phys. A, 18, $2751(1985)$

[SW 72] T. H. Schukan and H. A. Weidenmüller, Ann. Phys. (NY), 73, 108 (1972)

[Ta 90] K. T. Taylor in Atoms in Strong Fields, ed. C. A. Nicolaides, C. W. Clark and M. H. Nayfeh, NATO ASI Series B, 212, Plenum Press, New York (1990)

[To 91] S. Tomsovic, J. Phys. A, 24, L733 (1991)

[TS 91] G. Tanner, P. Scherer, E. B. Bogomonly, B. Eckhardt and D. Wintgen, Phys. Rev. Lett. 67, 2410 (1991)

[VW 29] J. von Neumann and E. P. Wigner, Z. Phys. 30, 467 (1929)

[We 92] H. A. Weidenmüller in Proceedings of the Eighth South African Summer School in Theoretical Physics on Chaos and Quantum Chaos, Eds. W.D. Heiss et al, Springer-Verlag (1992)

[WF 86] D. Wintgen and H. Friedrich, Phys. Rev. Lett. 57, 571 (1986)

D. Delande and J. C. Gay, ibid. 57, 2006 (1986)

[WF 87] D. Wintgen and H. Friedrich, Phys. Rev. A 36, 131 (1987)

[WF 90] H. Wu, H. Feng and M. Vallières, J. Phys. G 16, L149 (1990)

[WH 86] D. Wintgen, A. Holle, G. Wiebusch, J. Main, H. Friedrich and K. H. Welge, J. Phys. B 19, L557 (1986) 
[WH 89] D. Wintgen and A. Hönig, Phys. Rev. Lett. 63, 1467 (1989)

[Wi 51] E. P. Wigner, Ann. Math. Phys. 53, 30 (1951)

E. P. Wigner, ibid. 62, 584 (1955)

E. P. Wigner SIAM Rev. 9, 1 (1967)

[Wi 87a] D. Wintgen, Phys. Rev. Lett. 58, 1589 (1987)

[Wi 87b] D. Wintgen, J. Phys. B 20, L511 (1987)

[WKR 86] G. Wunner, M. Kost and H. Ruder, Phys. Rev. A 33, 1444 (1986)

[WM 88] D. Wintgen and H. Marxer, Phys. Rev. Lett. 61, 1803 (1988)

[WV 90] H. Wu, M. Vallières, D. Feng and D. W. L. Sprung, Phys. Rev. A, 42, 1027 (1990)

[WY 88] R. L. Waterland, J. Yuan, C. C. Martens, R. E. Gillilan and W. P. Reinhardt, Phys. Rev. Lett. 61, 2733 (1988)

[Za 85] G. M. Zaslavsky, Chaos in Dynamic Systems, Harwood Academic Publishers (1985)

[Ze 90] G. Zeller, Doctoral Thesis, University of Tübingen (1990)

[ZK 88] T. Zimmermann, H. Köppel, L. S. Cederbaum and G. Persch, Phys. Rev. Lett, 61, 3 (1988)

[ZKK 80] M. L. Zimmerman, M. M. Kash and D. Kleppner, Phys. Rev. Lett. 45, $1092(1980)$

[ZM 86] T. Zimmermann, H.-D. Meyer, H. Köppel and L. S. Cederbaum, Phys. Rev. A, 33, 4334 (1986)

[ZVW 83] M. R. Zirnbauer, J. J. M. Verbaarschot and H. A. Weidenmüller, Nucl. Phys. 411, 161 (1983)

$æ$ 